\title{
Cross-coupling of amide and amide derivatives to umbelliferone nonaflates: synthesis of coumarin derivatives and fluorescent materials
}

Shane M. Hickey, ${ }^{\mathrm{a}}$ Samuel O. Nitschke, ${ }^{\mathrm{a}}$ Martin. J. Sweetman, ${ }^{\mathrm{a}}$ Christopher J. Sumby, ${ }^{\mathrm{b}}$ Douglas A. Brooks, ${ }^{\mathrm{a}}$ Sally E. Plush, ${ }^{\mathrm{a}}$ and Trent D. Ashton, ${ }^{* \mathrm{c}, \mathrm{d}}$

${ }^{a}$ University of South Australia, Clinical Health Sciences, Cancer Research Institute, SA, 5000, Australia

${ }^{b}$ Department of Chemistry, The University of Adelaide, Adelaide, SA, 5005, Australia

${ }^{c}$ The Walter and Eliza Hall Institute of Medical Research, Parkville, 3052, Australia

${ }^{d}$ Department of Medical Biology, The University of Melbourne, Parkville 3010, Australia

E-mail: ashton.t@wehi.edu.au

\section{CONTENTS}

- ${ }^{1} \mathrm{H},{ }^{13} \mathrm{C}$ NMR, HRMS and HPLC spectra for all compounds (Figure S1-S157)

- Crystallography Data (Table S1)

- Gel Fabrication (Figure S158)

- Photophysical data of 25 in different solvents (Table S2)

- Normalised absorption and emission spectra (Figure S159-S176)

- References 


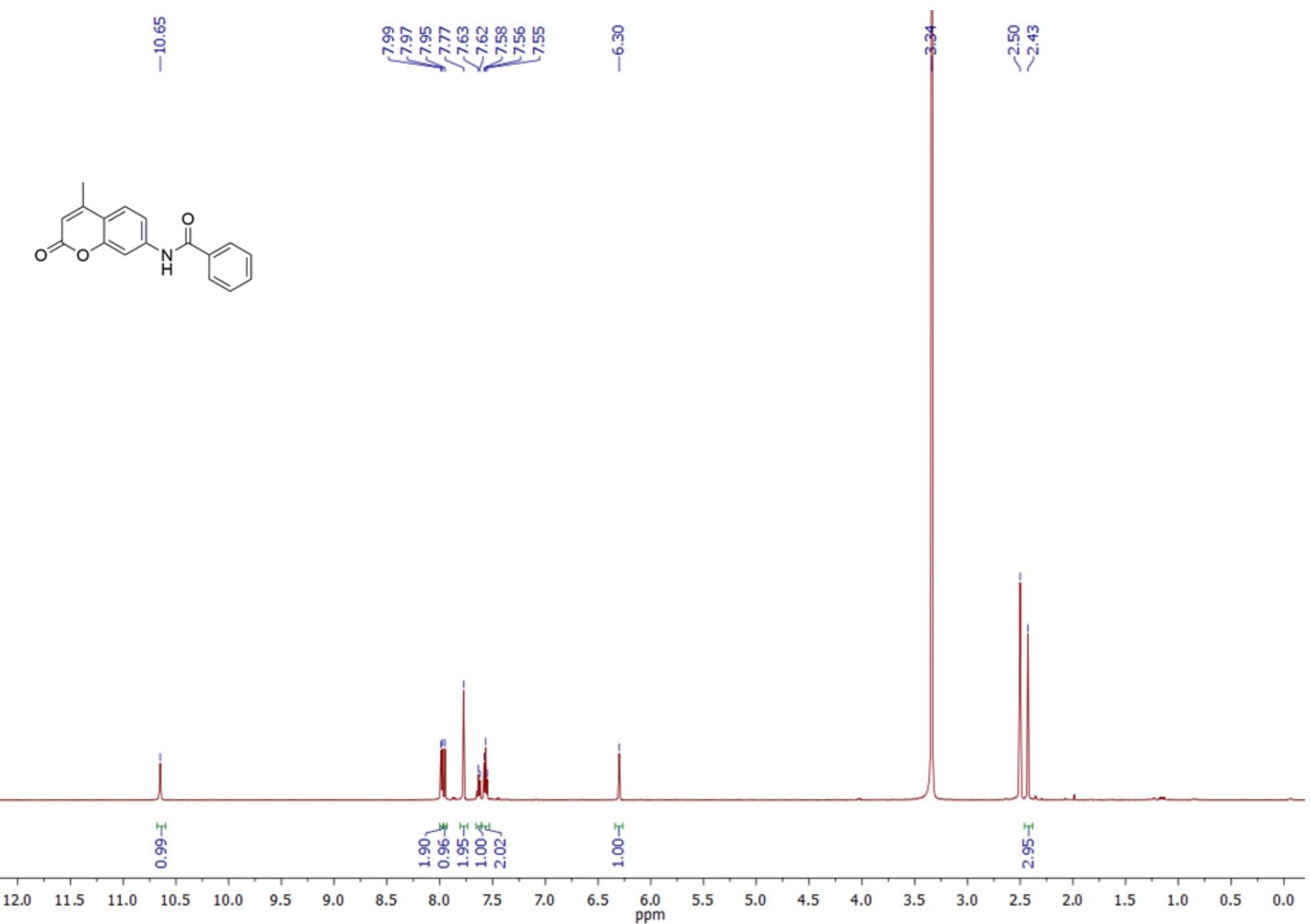

Figure S1: ${ }^{1} \mathrm{H}$ NMR $(500 \mathrm{MHz})$ spectrum of 1 in DMSO- $d_{6}$. 


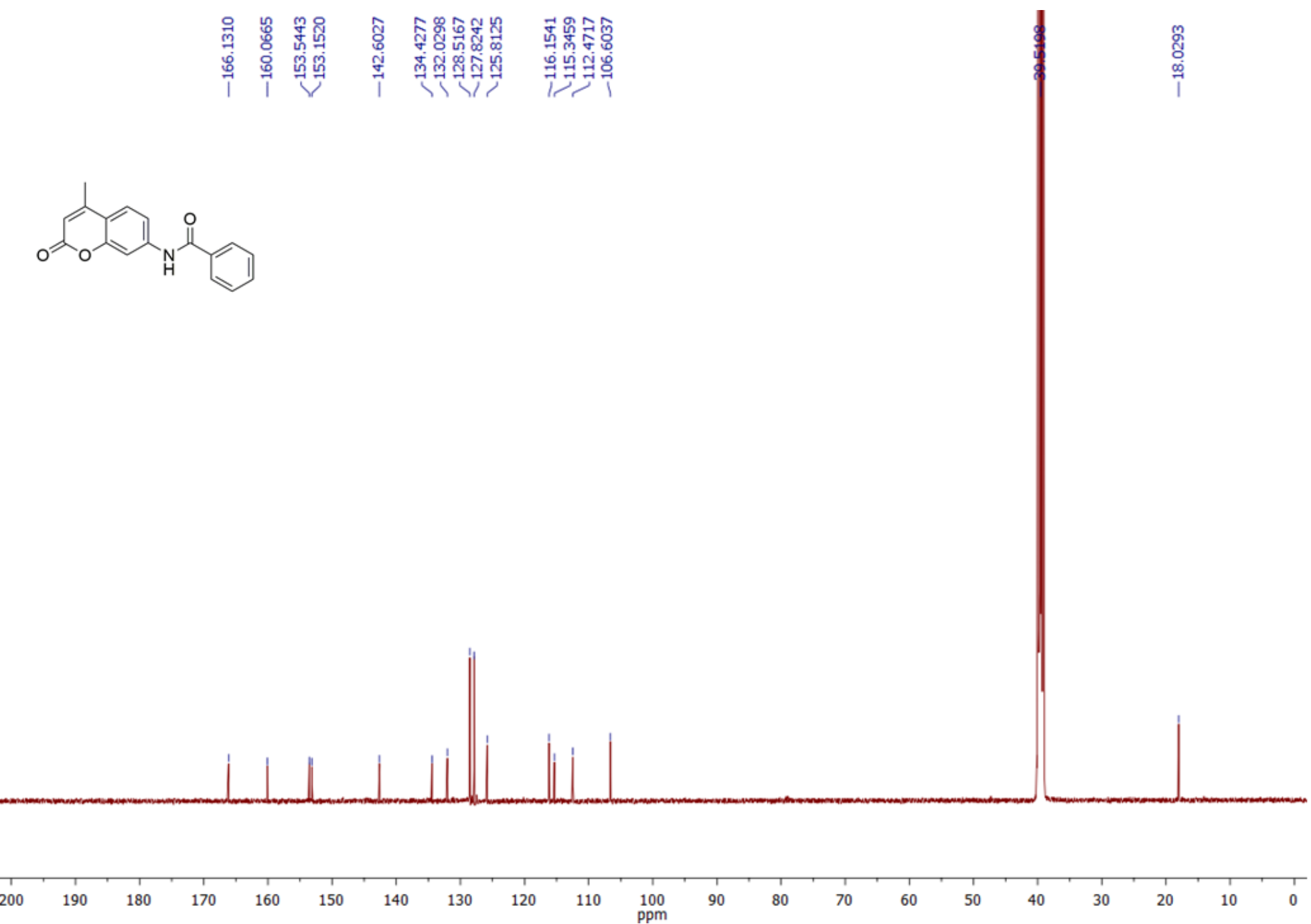

Figure S2: ${ }^{13} \mathrm{C}$ NMR $(125 \mathrm{MHz})$ spectrum of 1 in DMSO- $d_{6}$ 
+TOF MS: Exp 1, 0.1154 to 0.1432 min from Sample 1 (SMH09-031A) of SMH09-031A.wiff

$\mathrm{a}=7.02674898406060620 \mathrm{e}-004,10=7.55743060995075270 \mathrm{e}-001$ (DuoSpray ())

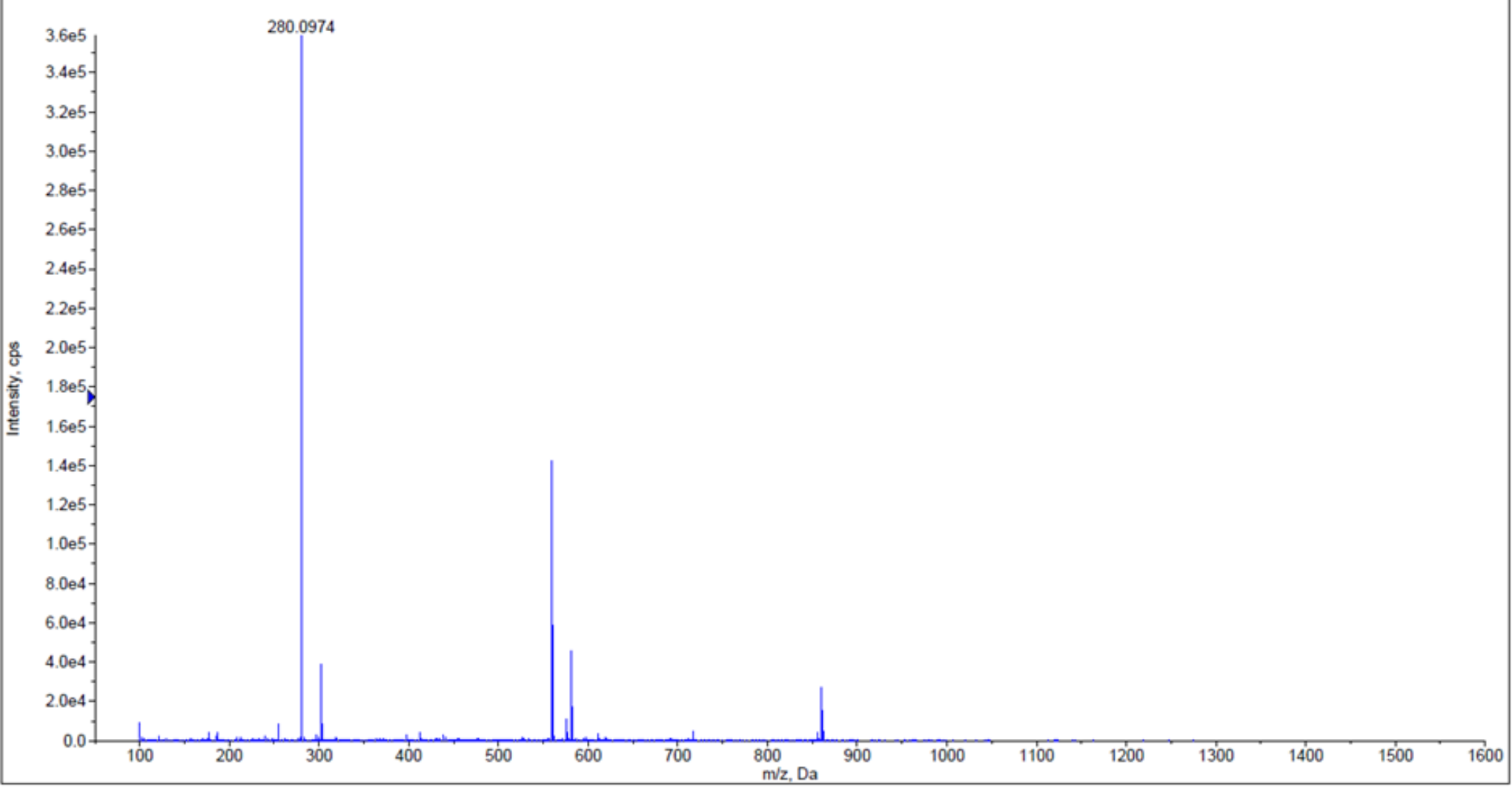

Figure S3: HRMS spectrum of 1. 
Chromatogram

mAU

SMH09-031A SMH09-031A.lcd

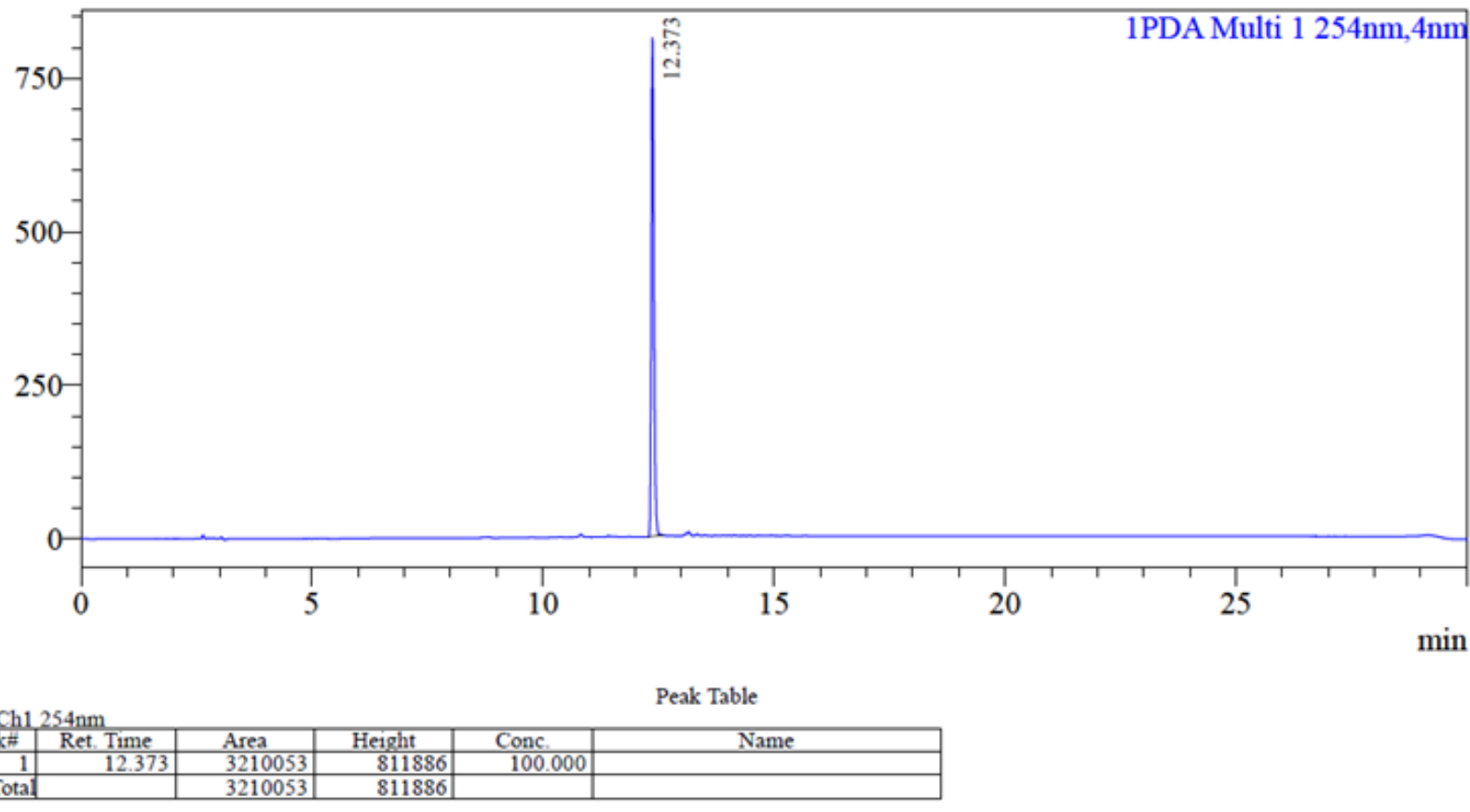

Figure S4: RP-HPLC trace of $\mathbf{1 .}$ 
ar
0
0
0

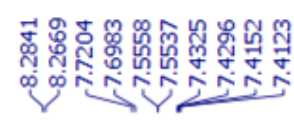

商

总

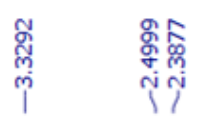
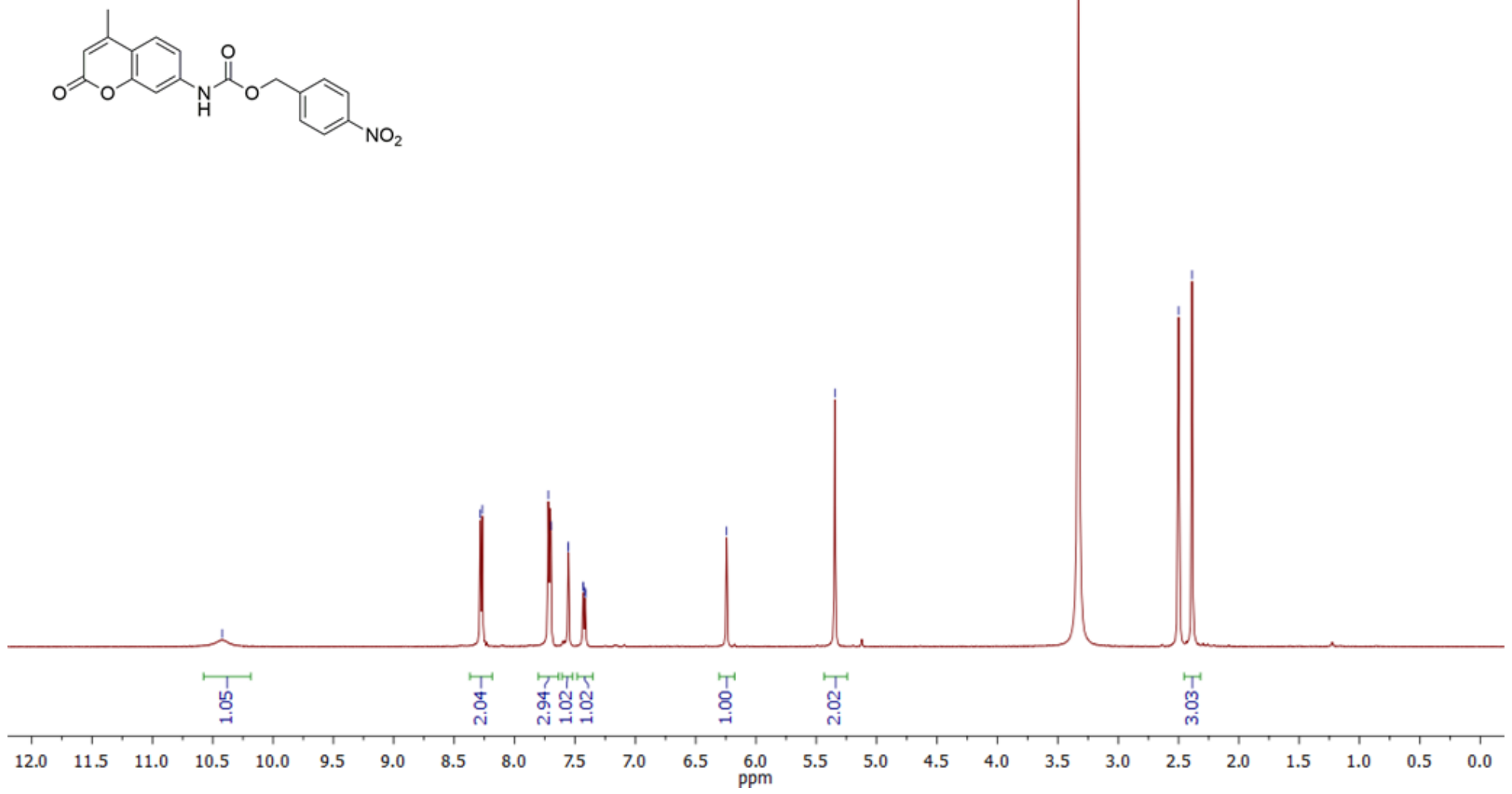

Figure S5: ${ }^{1} \mathrm{H}$ NMR (500 MHz) spectrum of 3 in DMSO- $d_{6}$.

S6 


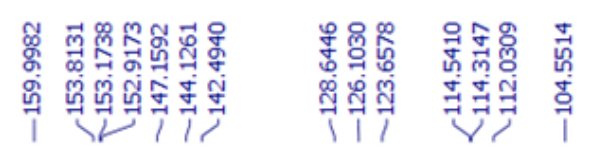

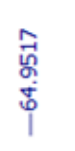
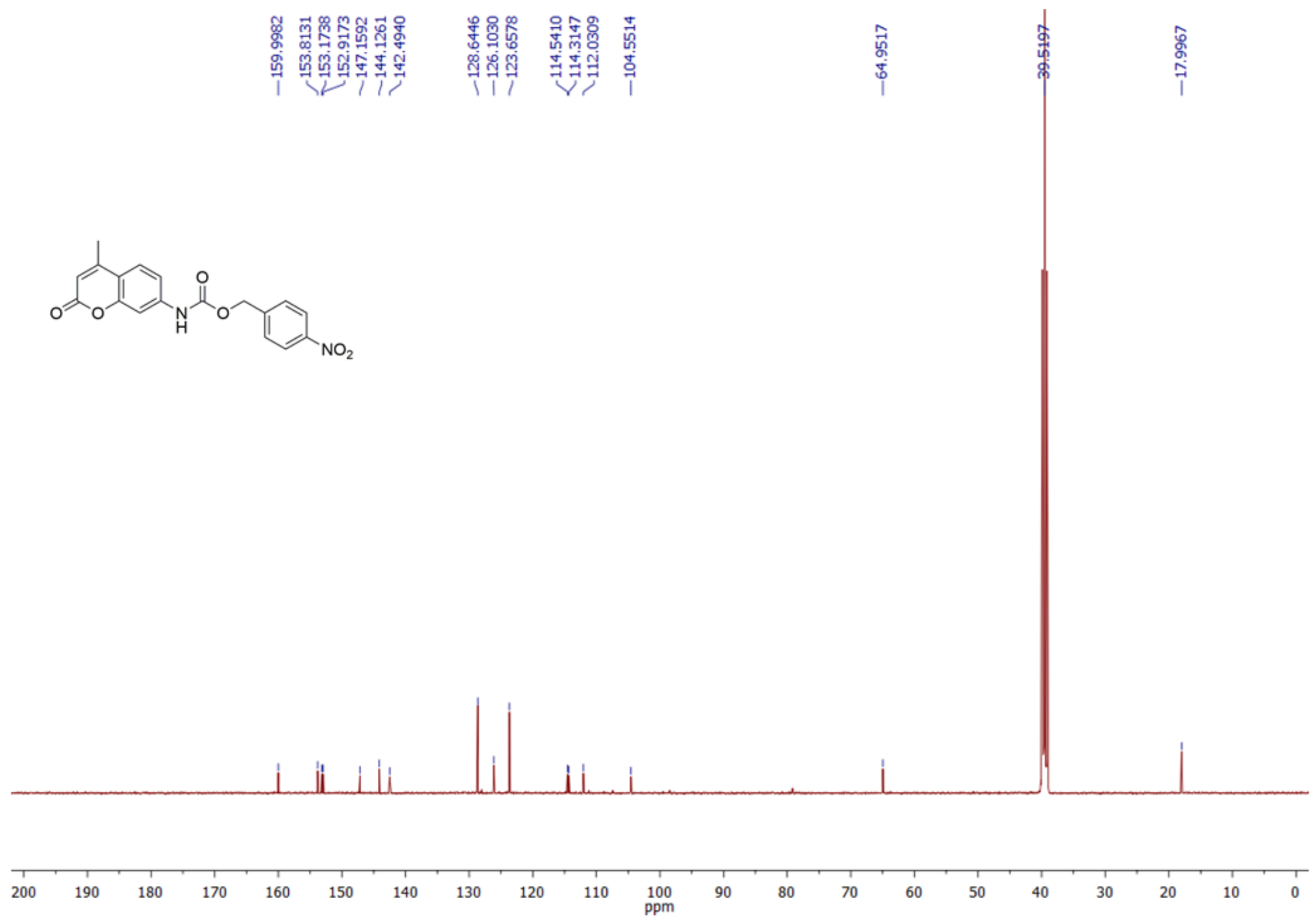

Figure S6: ${ }^{13} \mathrm{C}$ NMR $(125 \mathrm{MHz})$ spectrum of 3 in DMSO- $d_{6}$.

ST 
- +TOF MS: Exp 1, 0.4027 to $0.4212 \mathrm{~min}$ from Sample 1 (SMH12-083C) of SMH12-083C.wiff different calibrations (DuoSpray ())

Max. $8360.0 \mathrm{cps}$

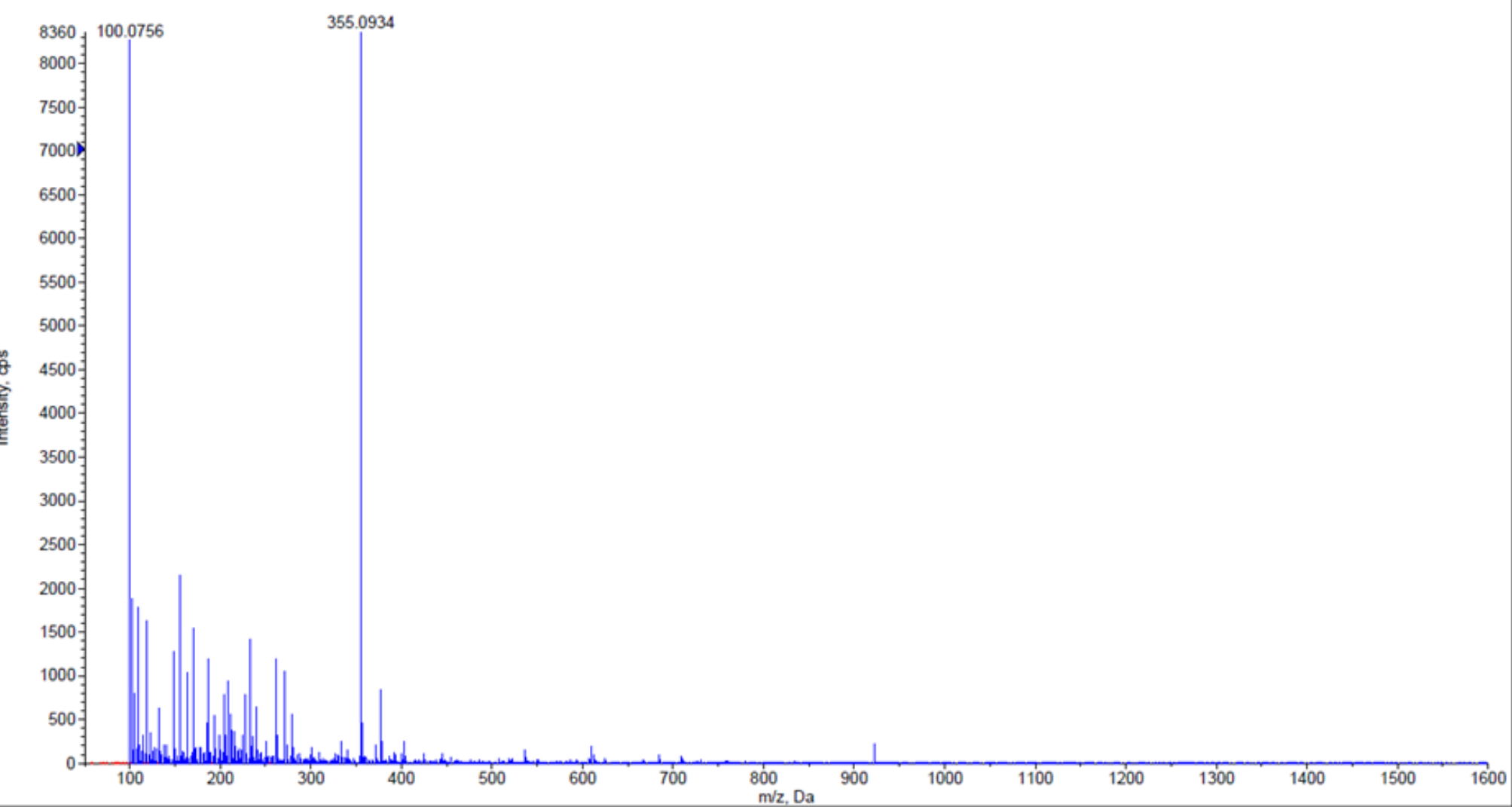

Figure S7: HRMS spectrum of 3 . 
Chromatogram

mAU

SMH12-083C SMH12-083C.lcd

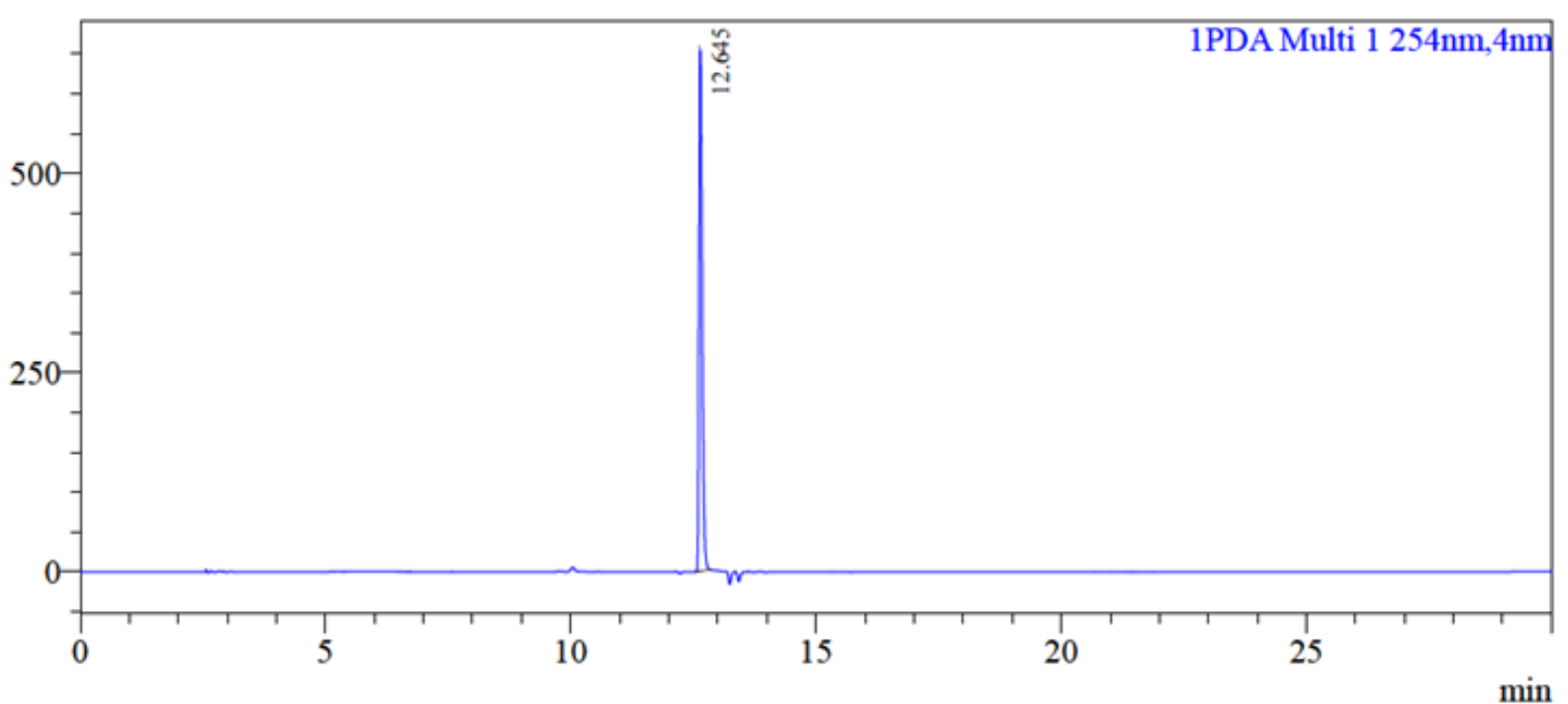

Peak Table

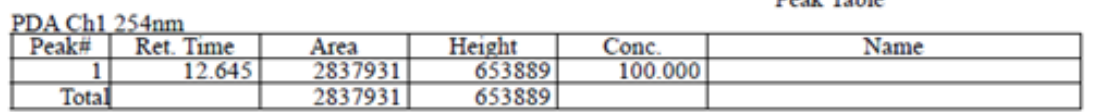

Figure S8: RP-HPLC trace of 3. 


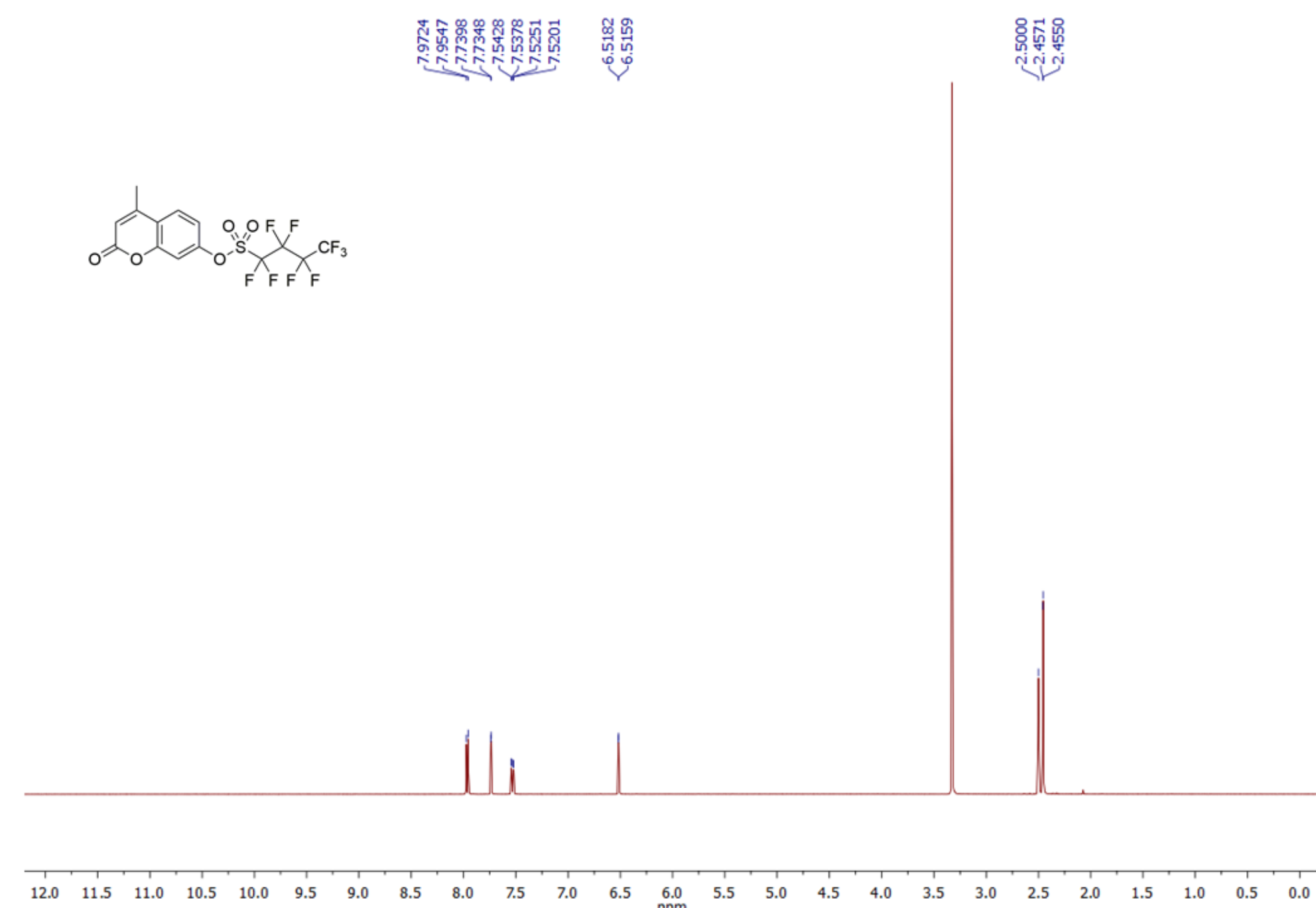

Figure S9: ${ }^{1} \mathrm{H}$ NMR $(500 \mathrm{MHz})$ spectrum of 5 in DMSO- $d_{6}$. 


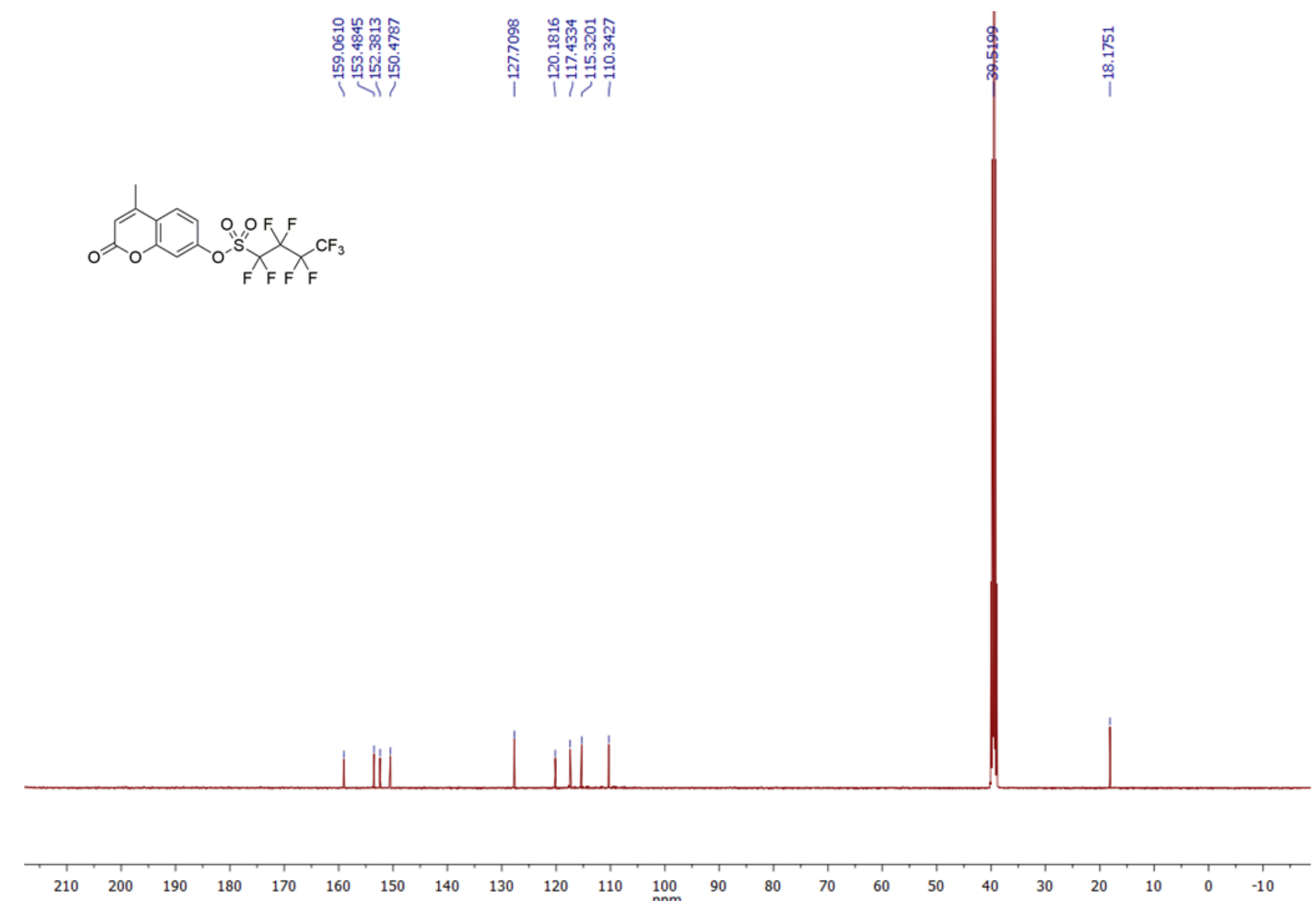

Figure S10: ${ }^{13} \mathrm{C}$ NMR $(125 \mathrm{MHz})$ spectrum of 5 in DMSO- $d_{6}$. 


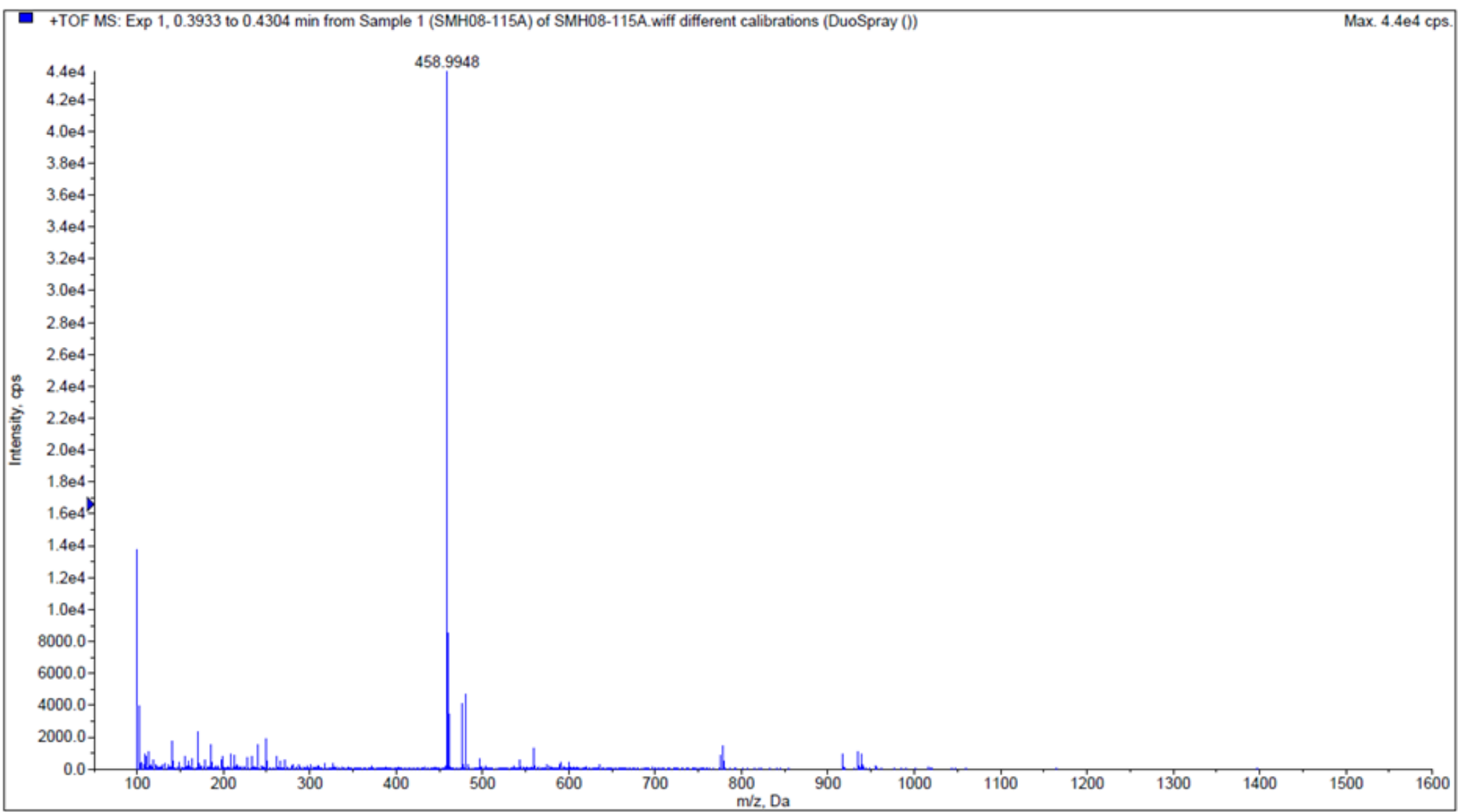

Figure S11: HRMS spectrum of 5 . 
Chromatogram

SMH08-115A SMH08-115A.lcd

mAU

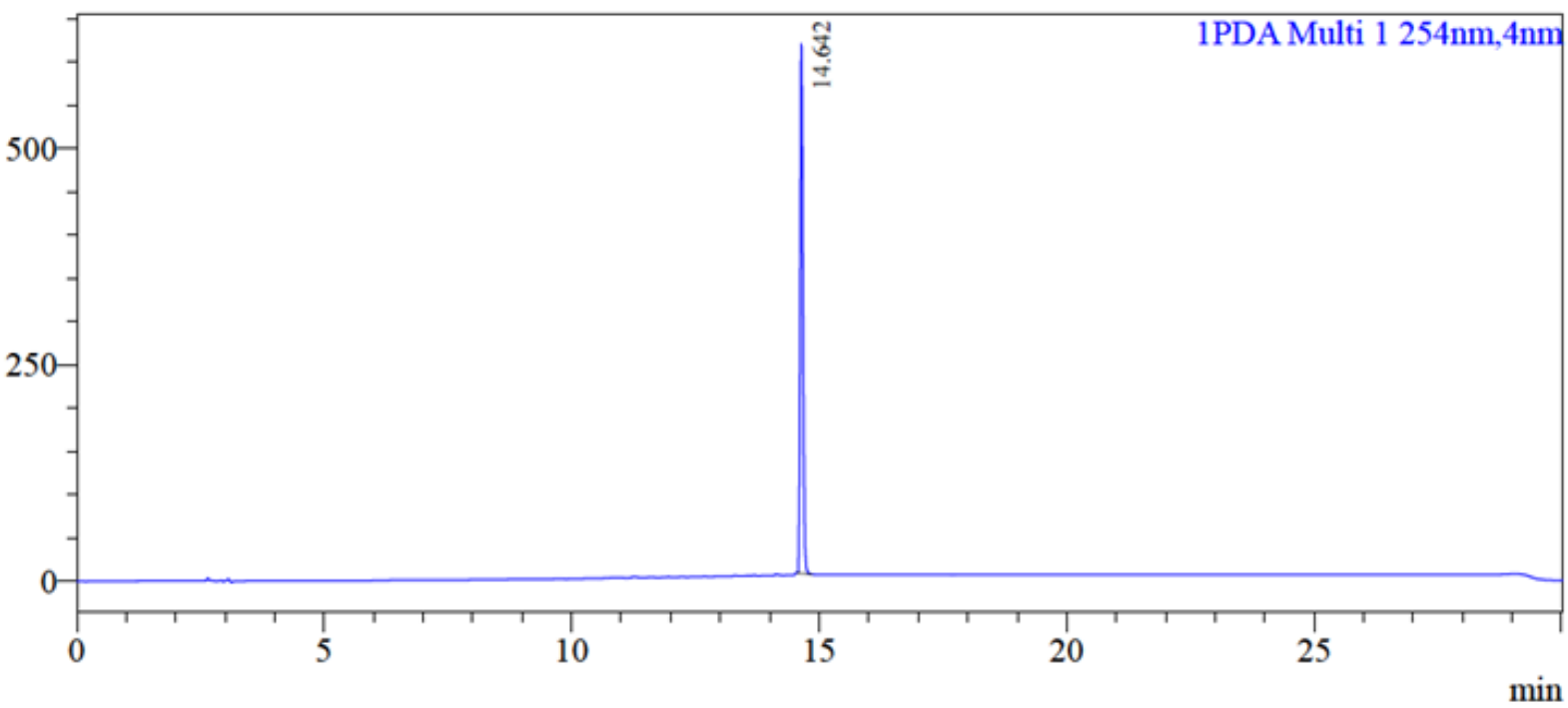

Peak Table

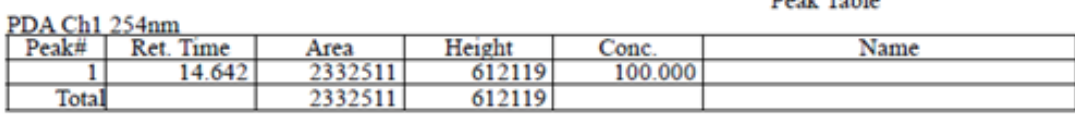

Figure S12: RP-HPLC trace of 5 
䒿

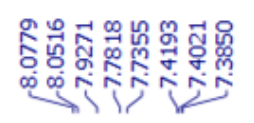

㖞

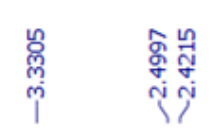
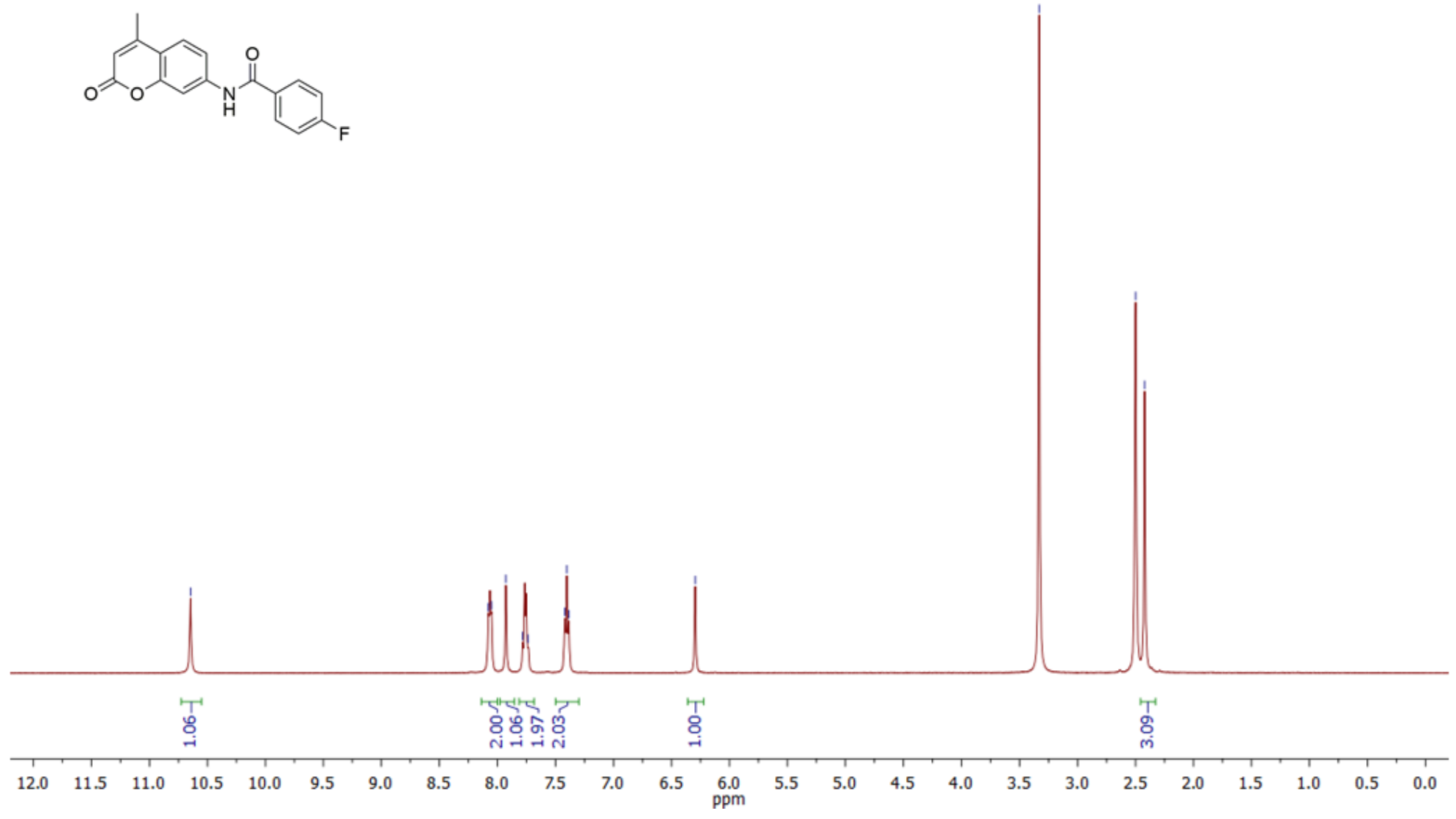

Figure S13: ${ }^{1} \mathrm{H}$ NMR $(500 \mathrm{MHz})$ spectrum of 6 in DMSO- $d_{6}$.

S14 


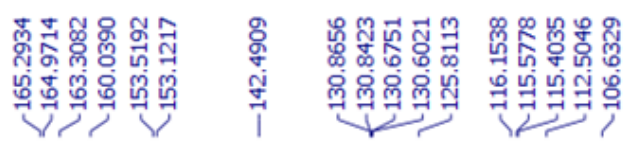
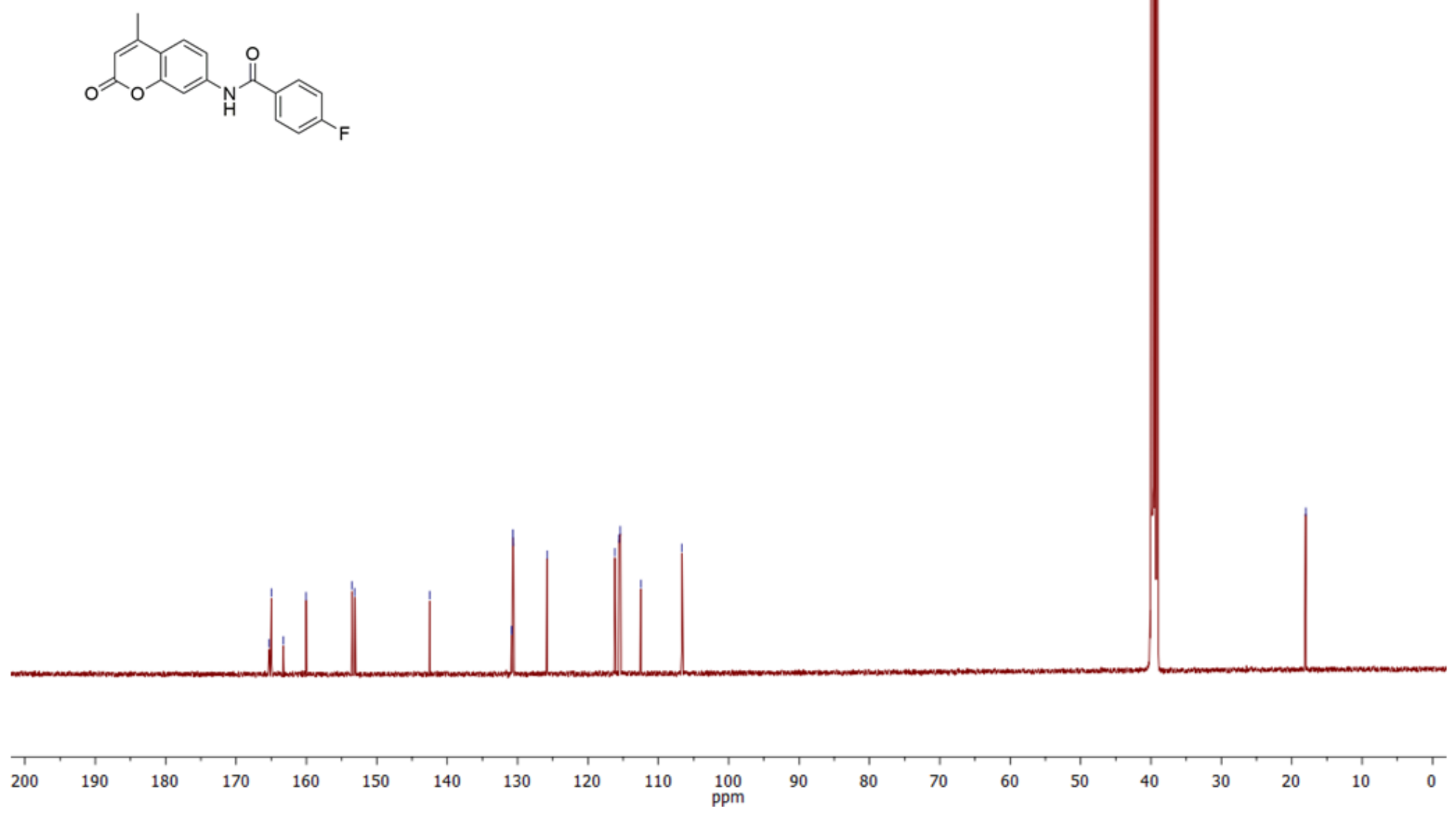

Figure S14: ${ }^{13} \mathrm{C}$ NMR $(125 \mathrm{MHz})$ spectrum of 6 in DMSO- $d_{6}$.

$\mathrm{S} 15$ 


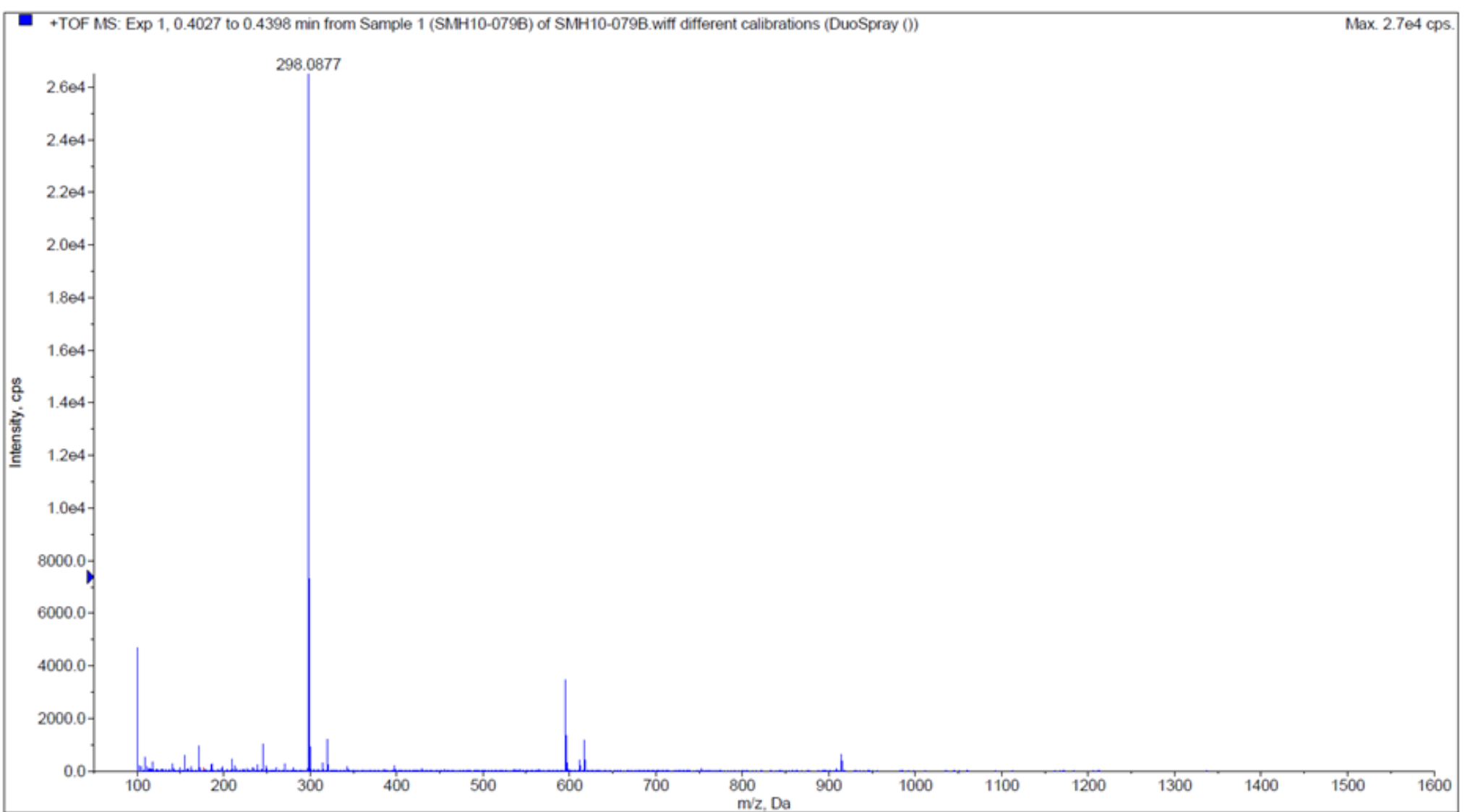

Figure S15: HRMS spectrum of 6 
Chromatogram

mAU

SMH10-079B SMH10-079B.lcd

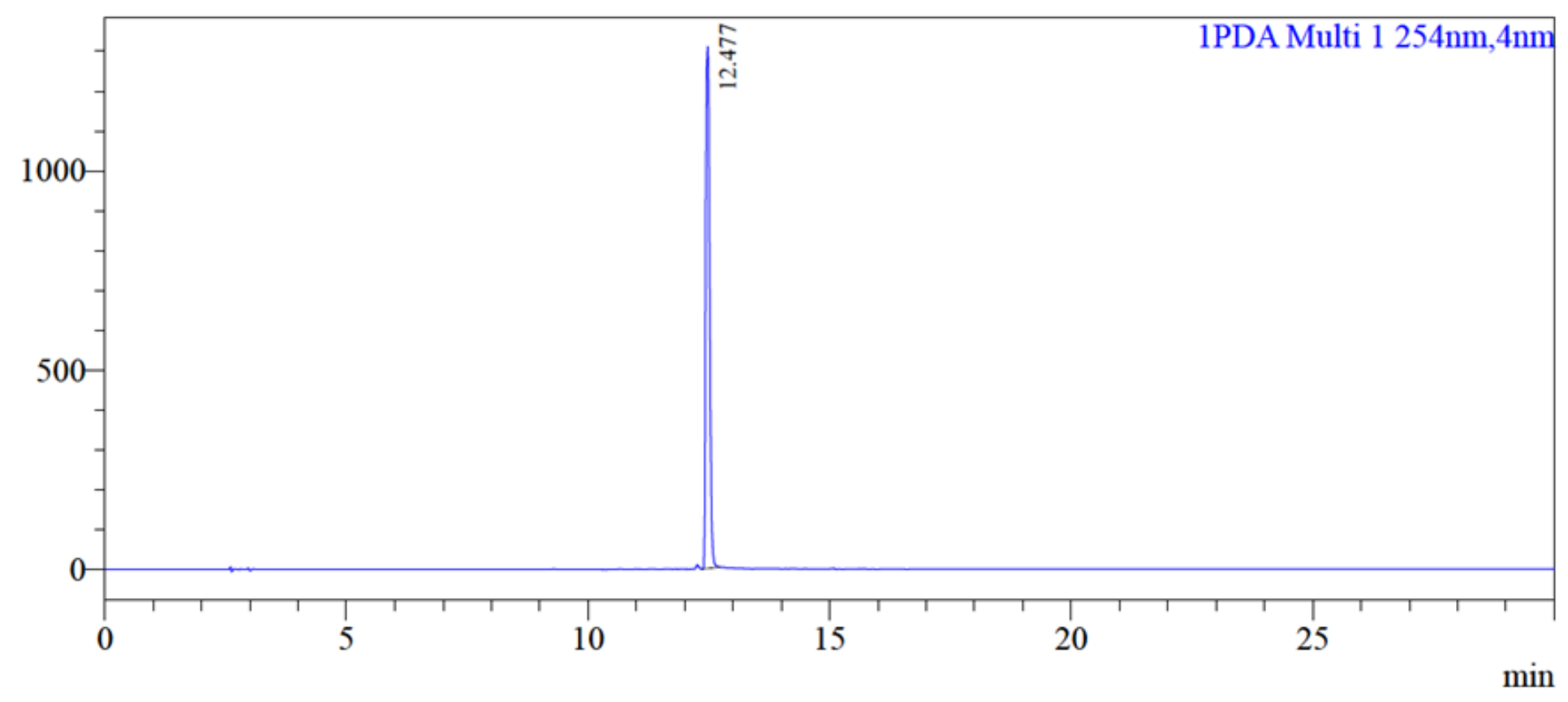

Peak Table

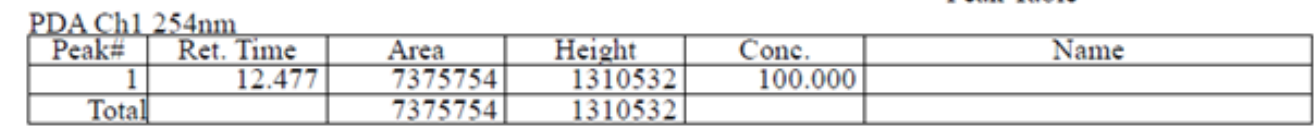

Figure S16: RP-HPLC trace of 6. 
ิㅗㅇ

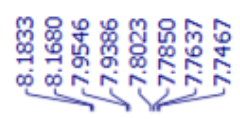

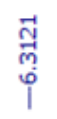

๖ัญ
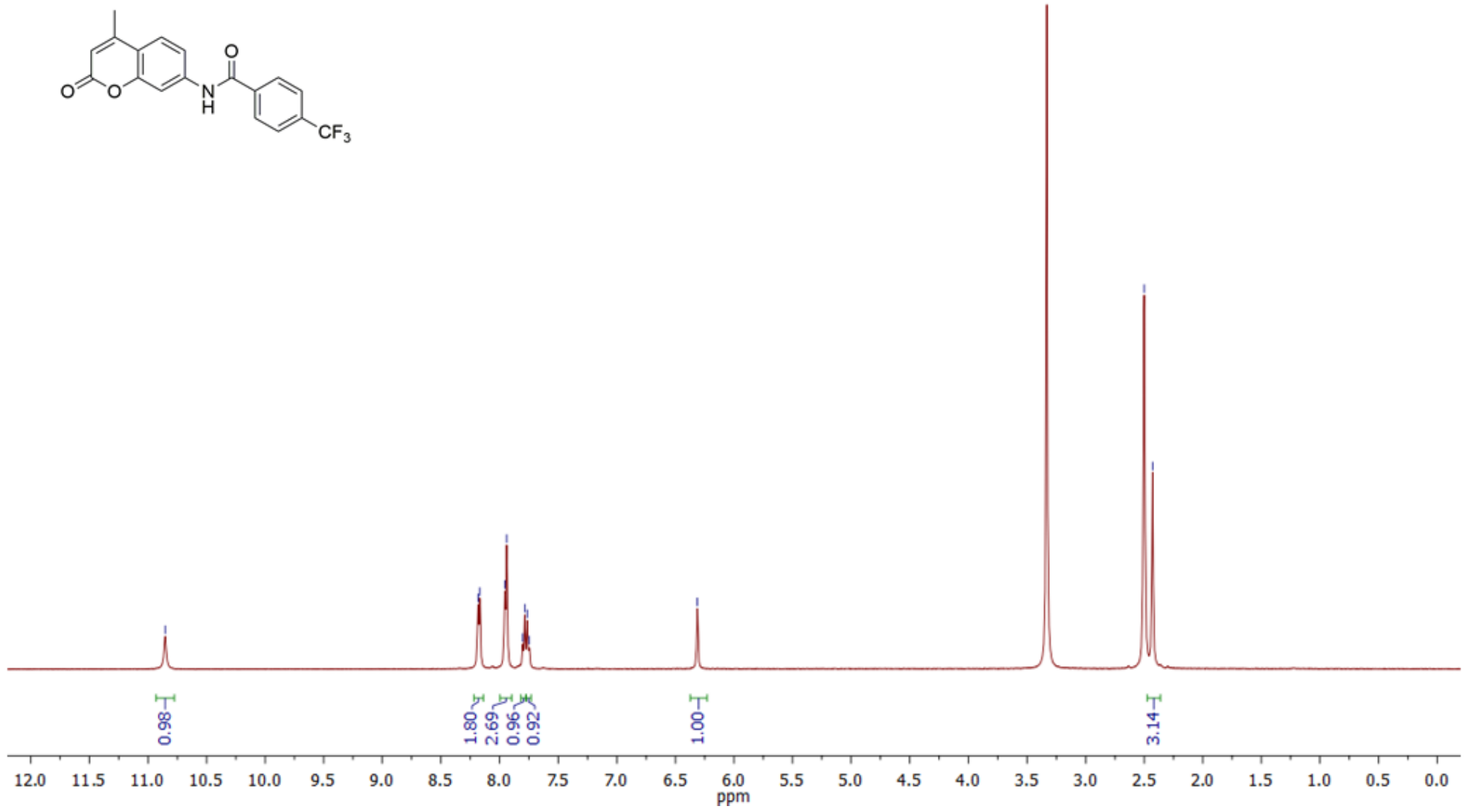

Figure S17: ${ }^{1} \mathrm{H}$ NMR $(500 \mathrm{MHz})$ spectrum of 7 in DMSO- $d_{6}$.

$\mathrm{S} 18$ 


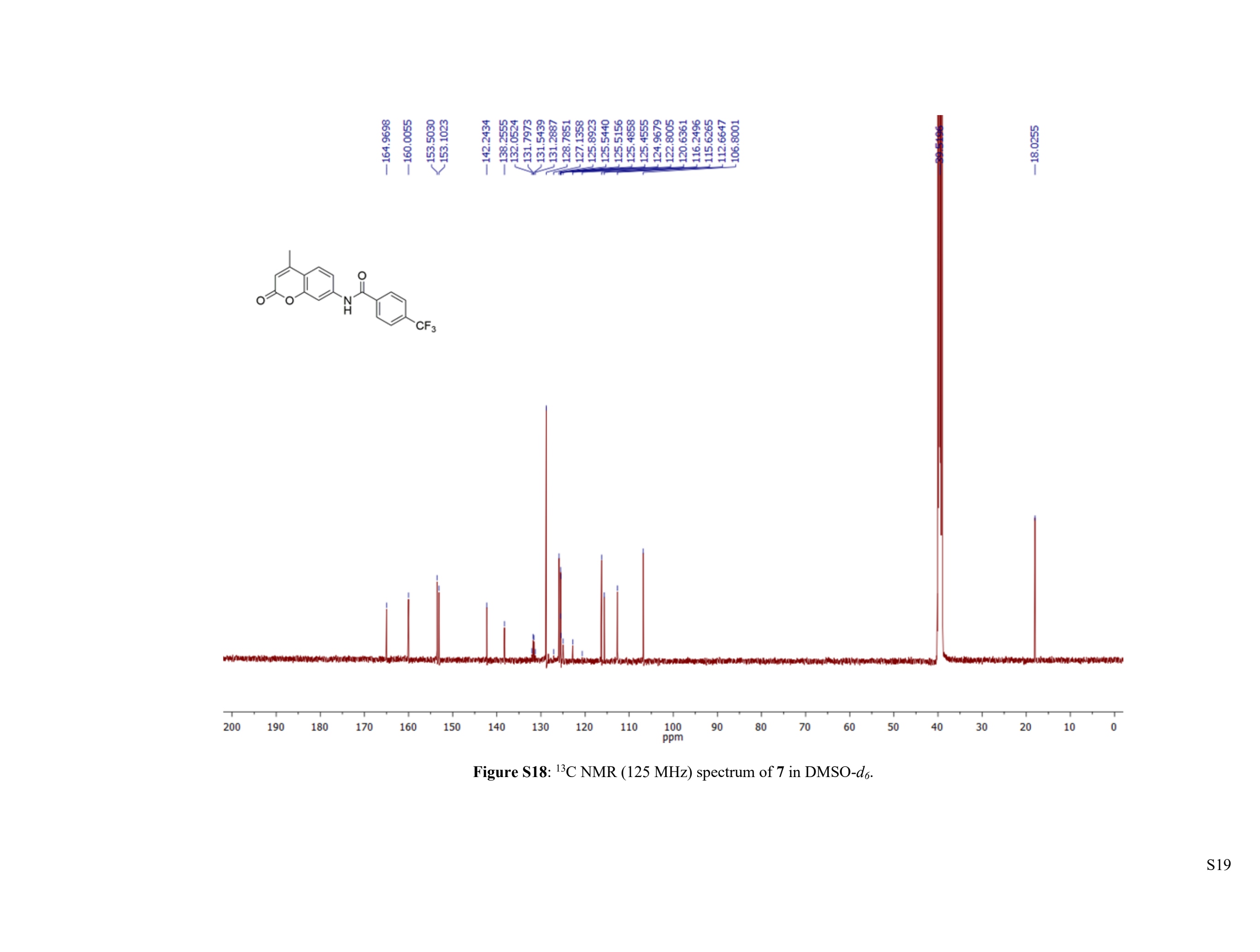




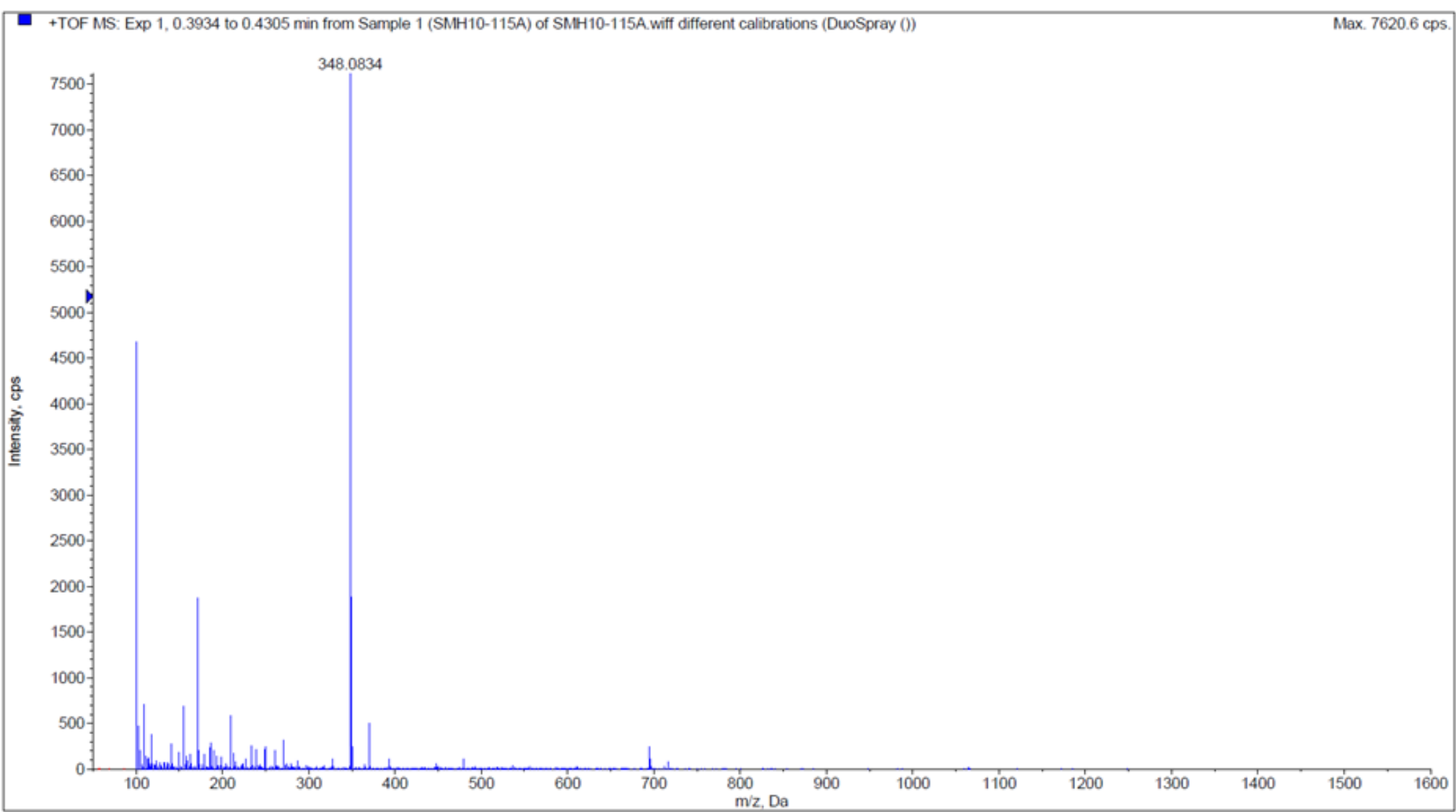

Figure S19: HRMS spectrum of 7 . 
Chromatogram

mAU

SMH10-115A SMH10-115A.lcd

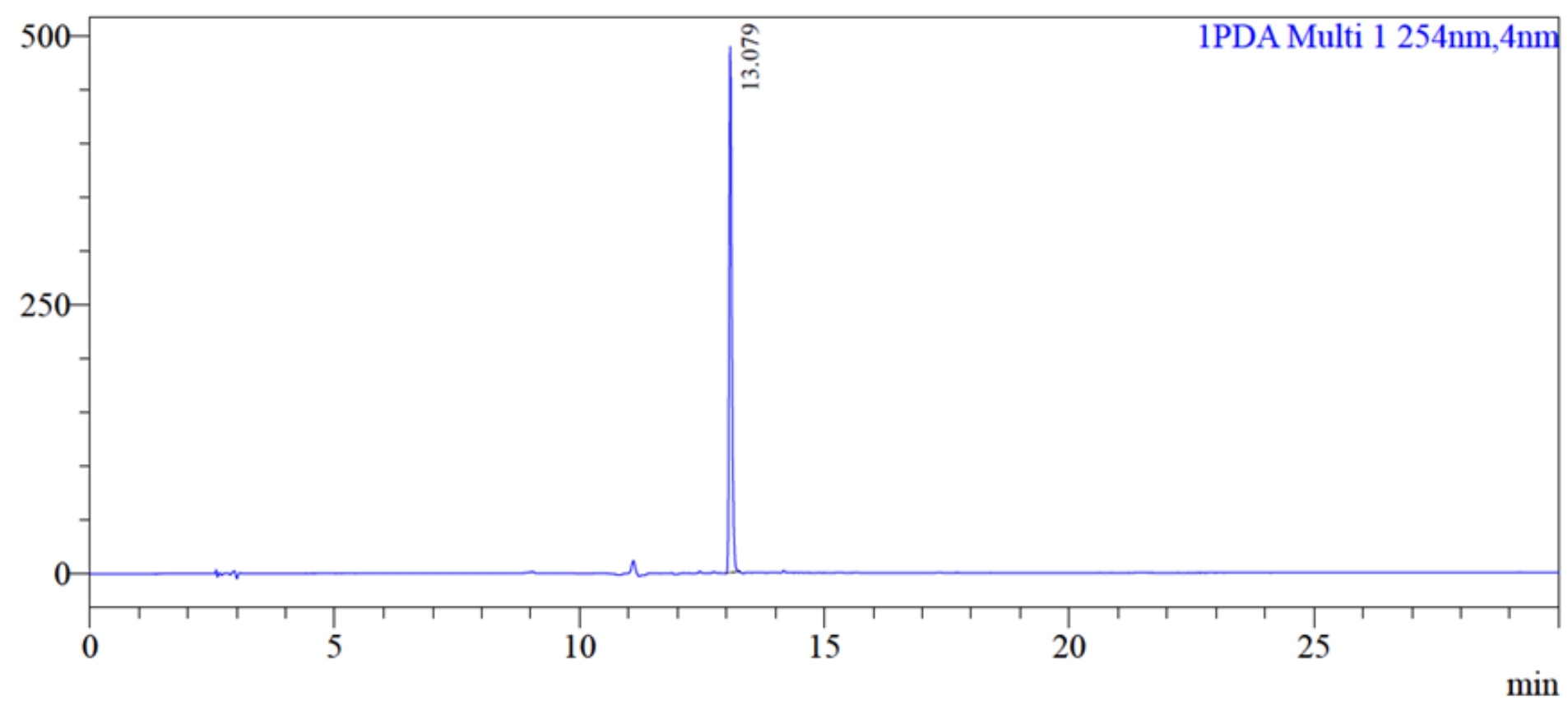

Peak Table

PDA Ch1 $254 \mathrm{~nm}$
\begin{tabular}{|r|r|r|r|r|r|}
\hline Peak= & Ret. Time & Area & Height & Conc. & Name \\
\hline 1 & 13.079 & 1830663 & 489019 & 100.000 & \\
\hline Total & & 1830663 & 489019 & & \\
\hline
\end{tabular}

Figure S20: RP-HPLC trace of 7. 


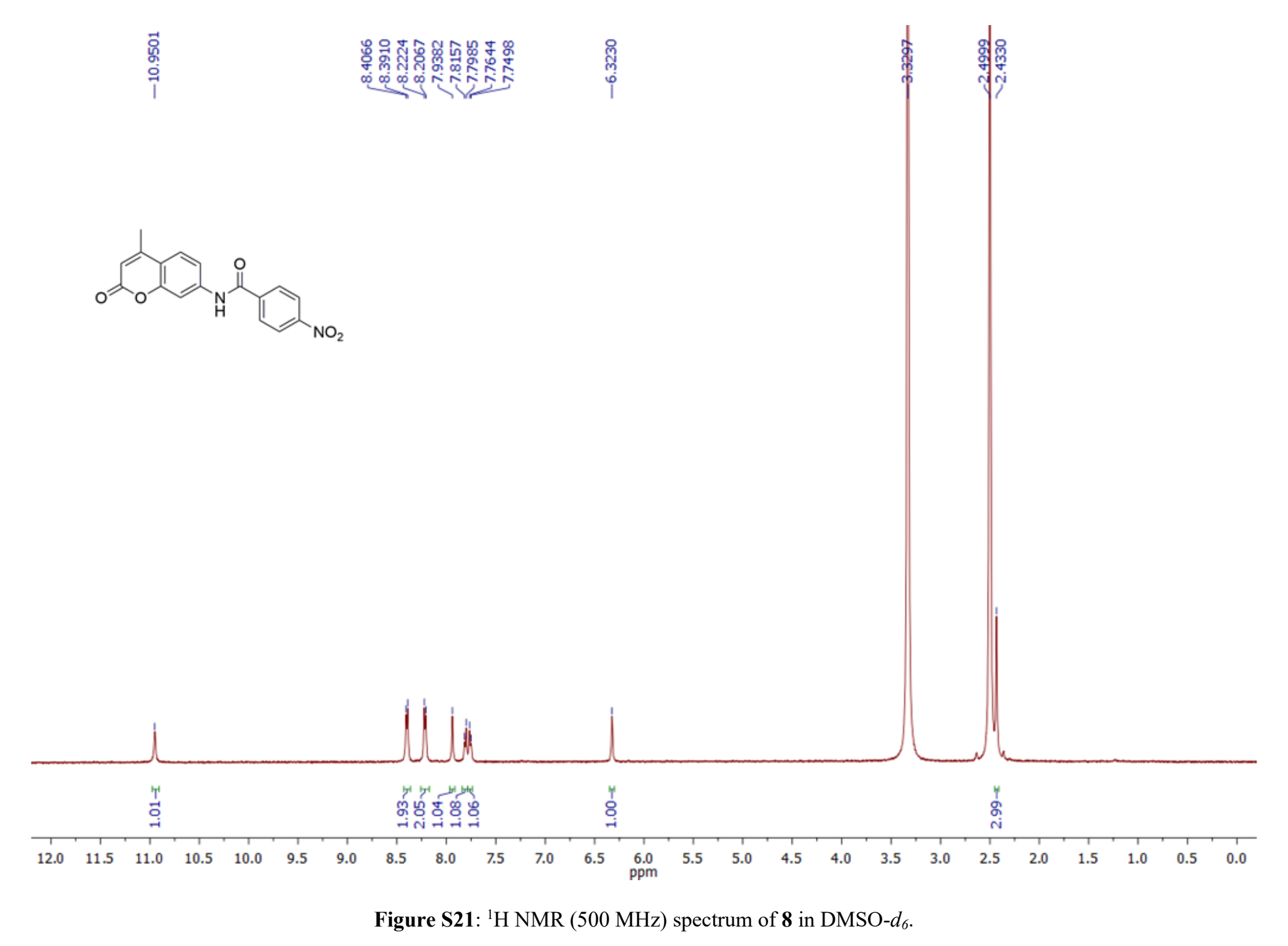




$$
\underline{\underline{y-1}}
$$


-TOF MS: Exp 1, 0.4027 to 0.4305 min from Sample 2 (SMH11-009B) of SMH11-0098. wiff different calibrations (DuoSpray ())

Max. $1845.8 \mathrm{cps}$.

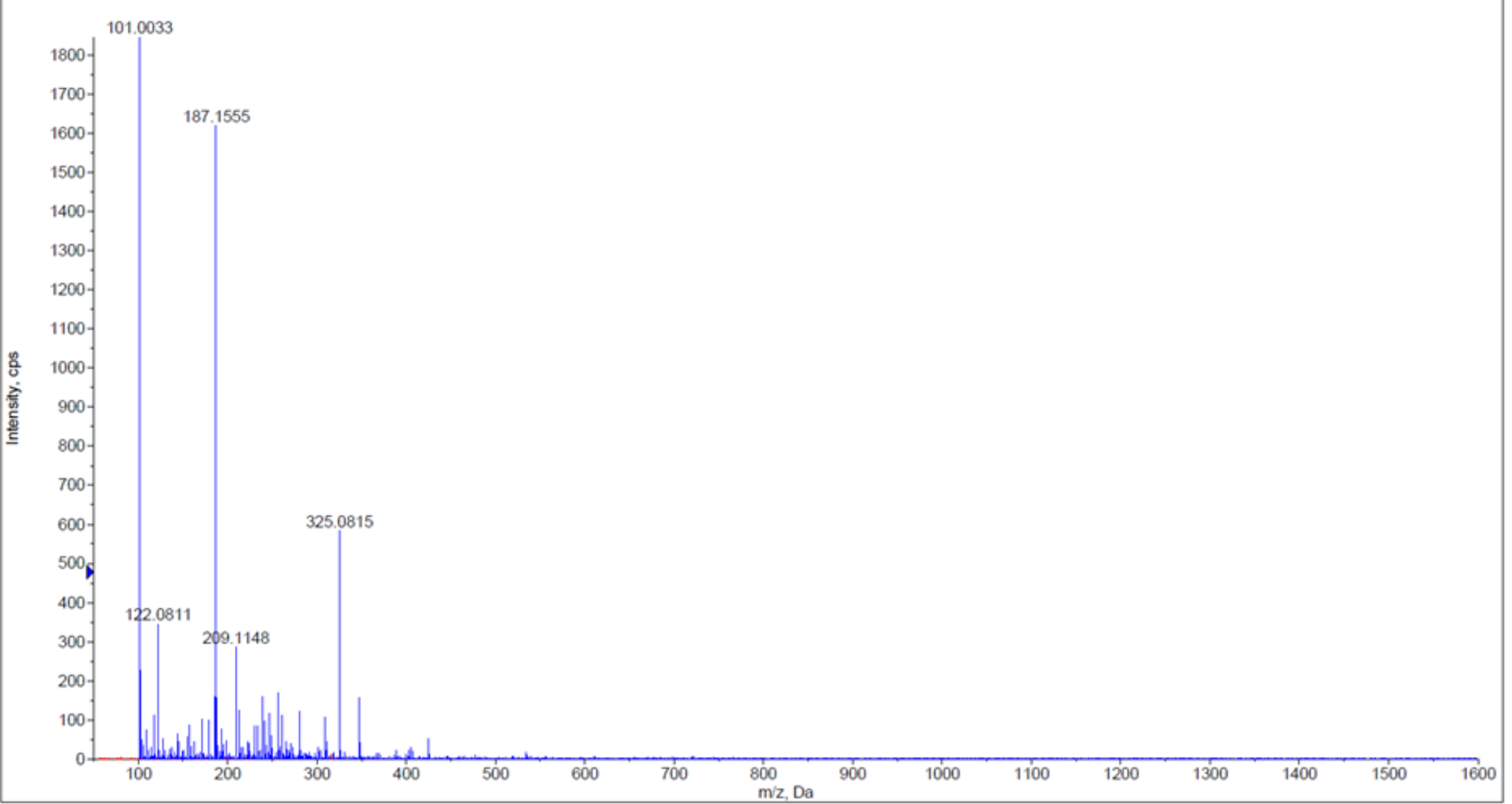

Figure S23: HRMS spectrum of 8 
Chromatogram

SMH11-009B SMH11-009B.lcd

mAU

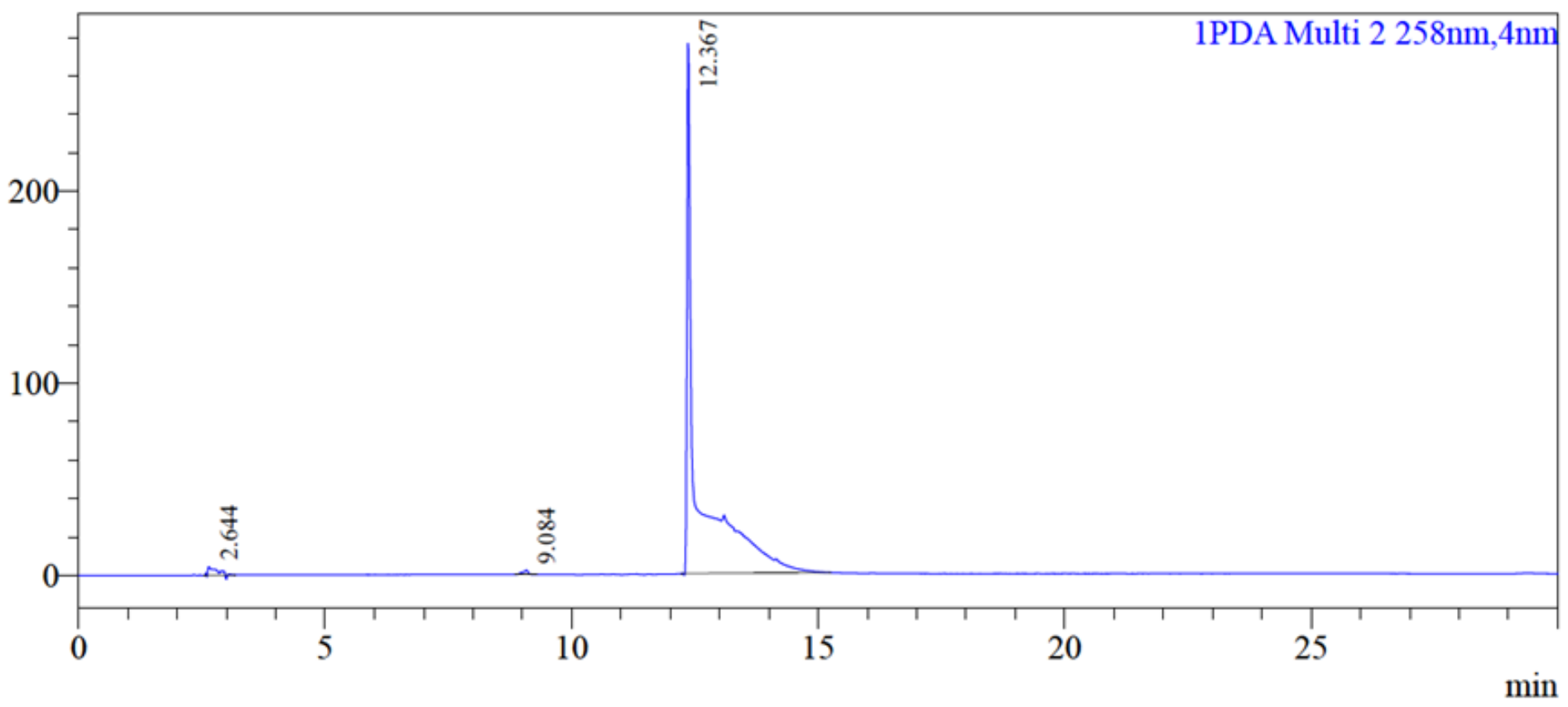

Peak Table

PDA Ch2 $258 \mathrm{~nm}$
\begin{tabular}{|r|r|r|r|r|l|}
\hline Peak\# & Ret. Time & \multicolumn{1}{|c|}{ Area } & Height & Conc. & \\
\hline 1 & 2.644 & 52315 & 4297 & 1.427 & \\
\hline 2 & 9.084 & 17949 & 2344 & 0.490 & \\
\hline 3 & 12.367 & 3595204 & 276184 & 98.083 & \\
\hline Total & & 3665468 & 282825 & & \\
\hline
\end{tabular}

Figure S24: HPLC spectrum of 8 . 


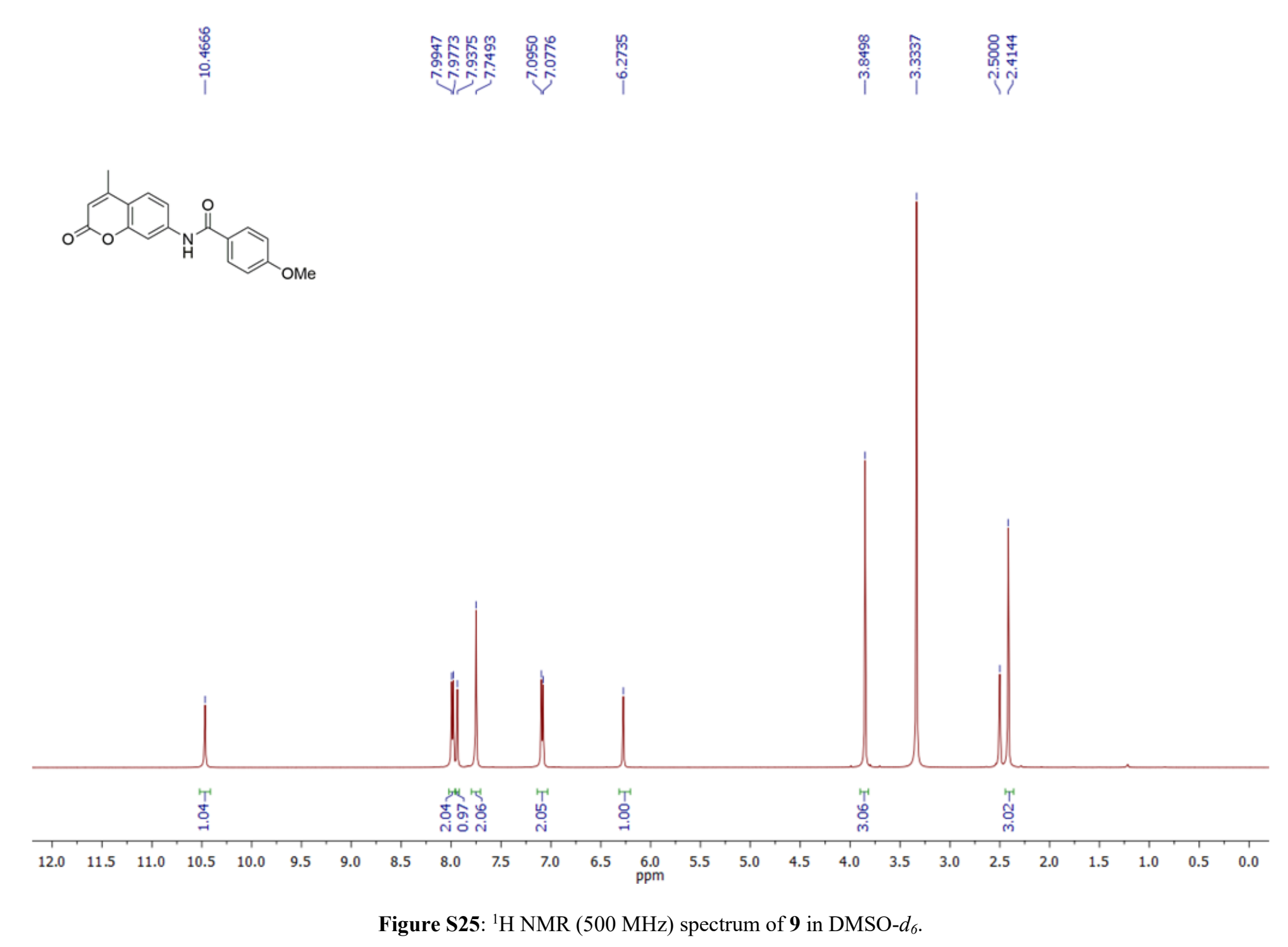




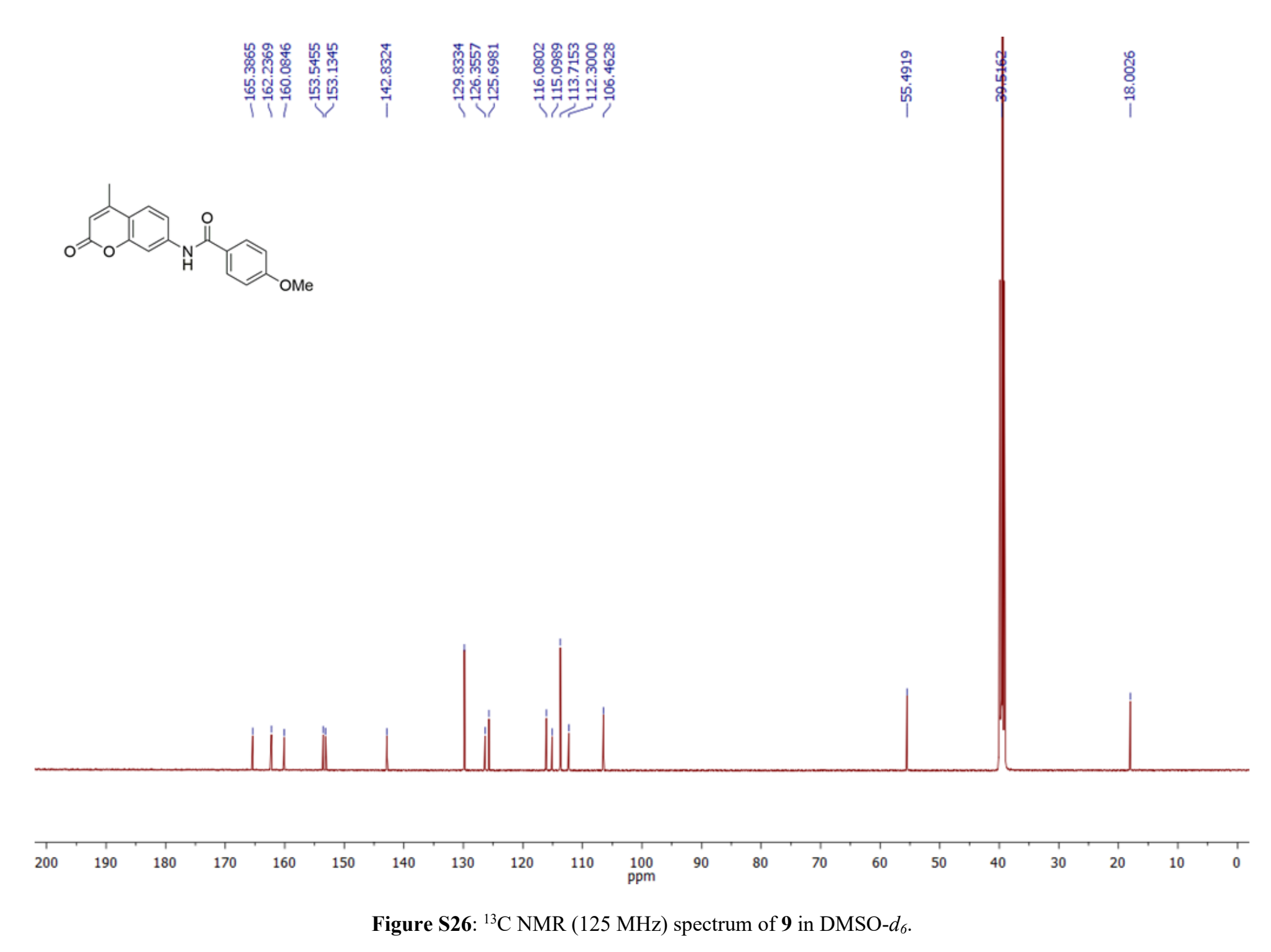


+TOF MS: 0.0972 to 0.1065 min from Sample 4 (SON04-053A) of SON04-053.witl

$a=7.02667138678842090 \mathrm{e}-004,0=8.46313277395368990 \mathrm{e}-001$ (DuoSpray ())

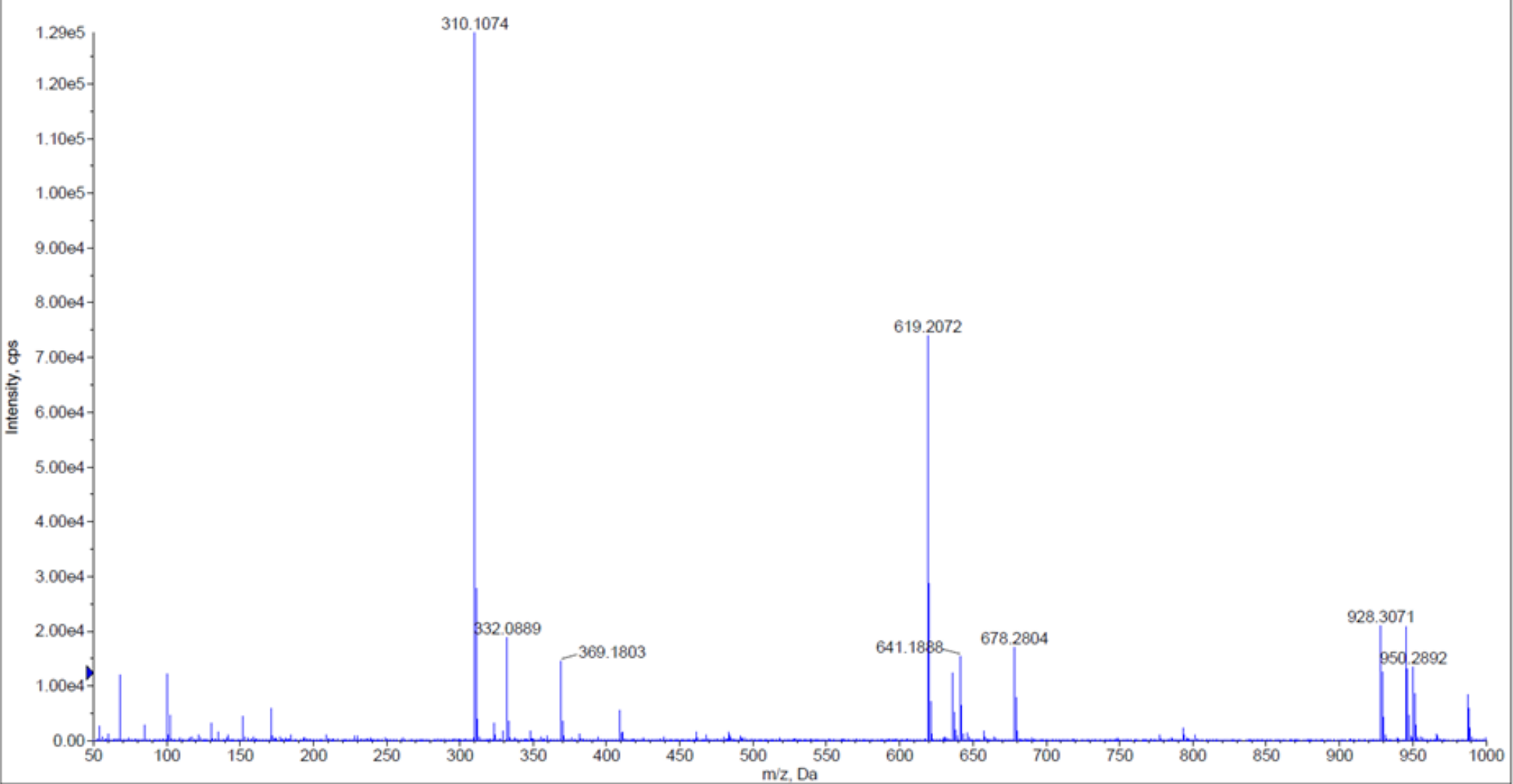

Figure S27: HRMS spectrum for 9 
Chromatogram

SON04-053A SON04-053A.led

mAU

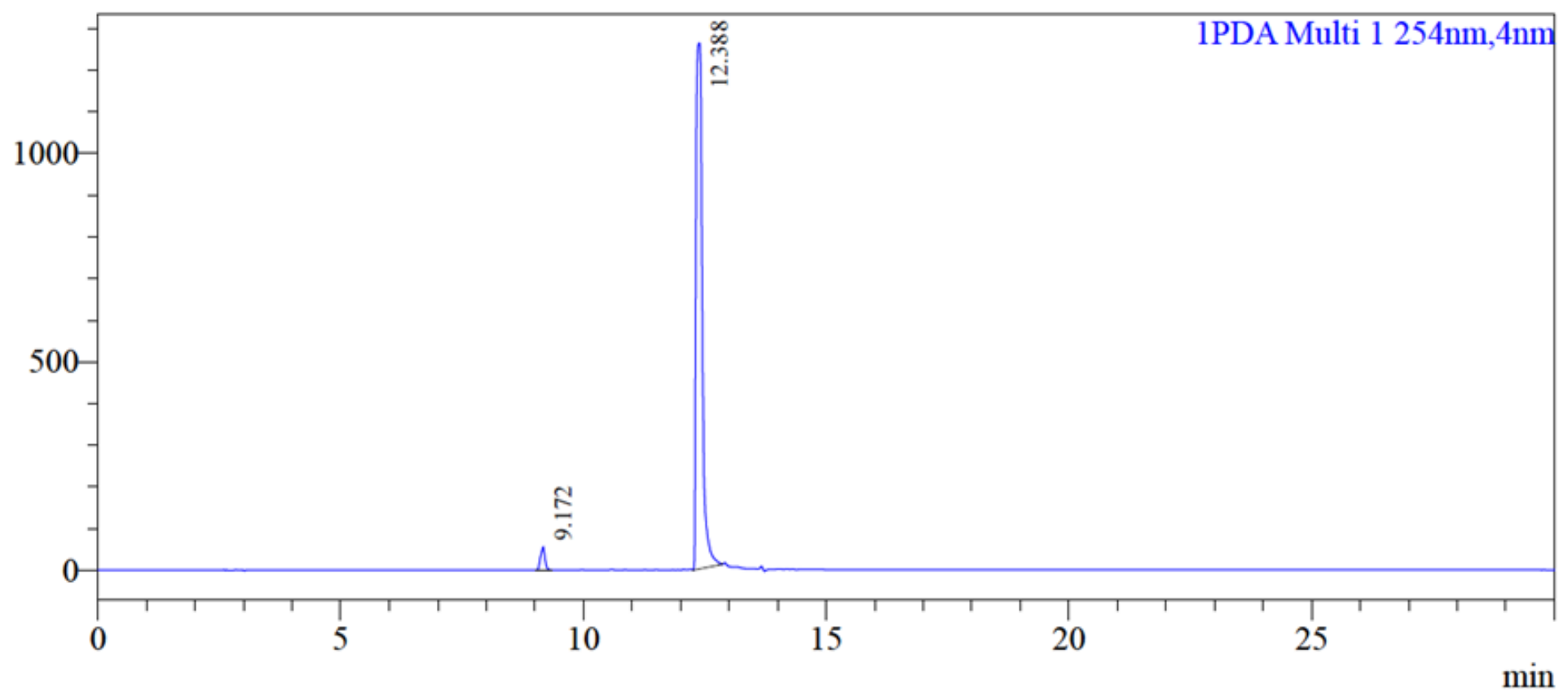

Peak Table

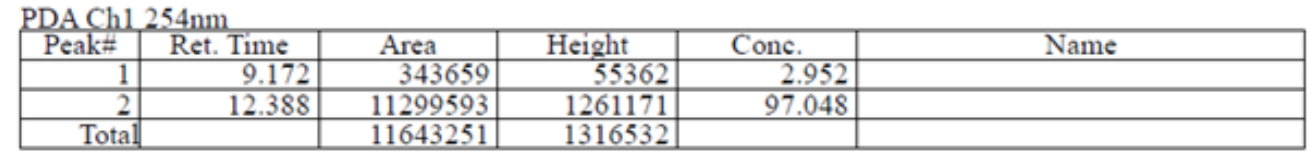

Figure S28: HPLC trace for 9. 


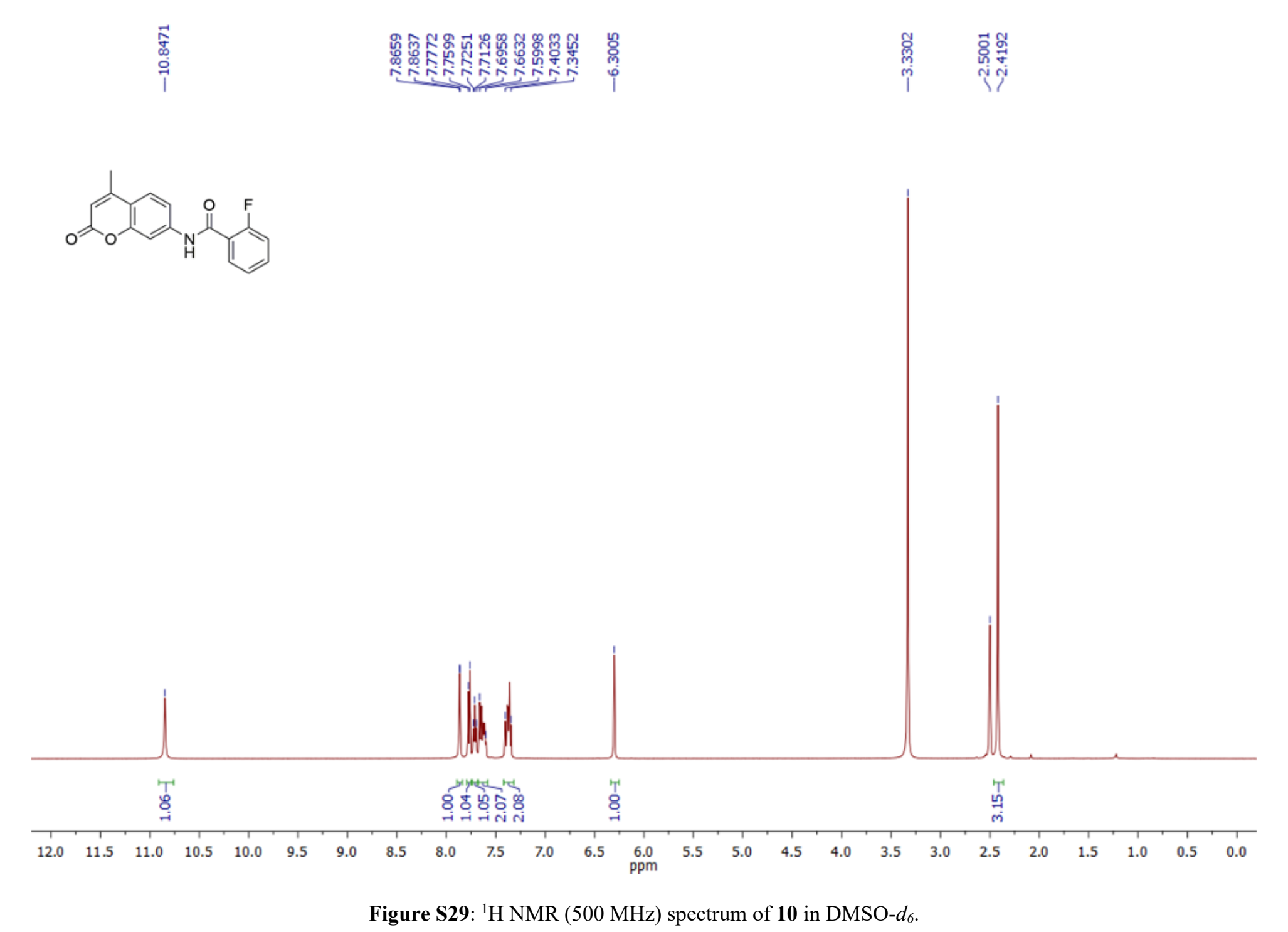




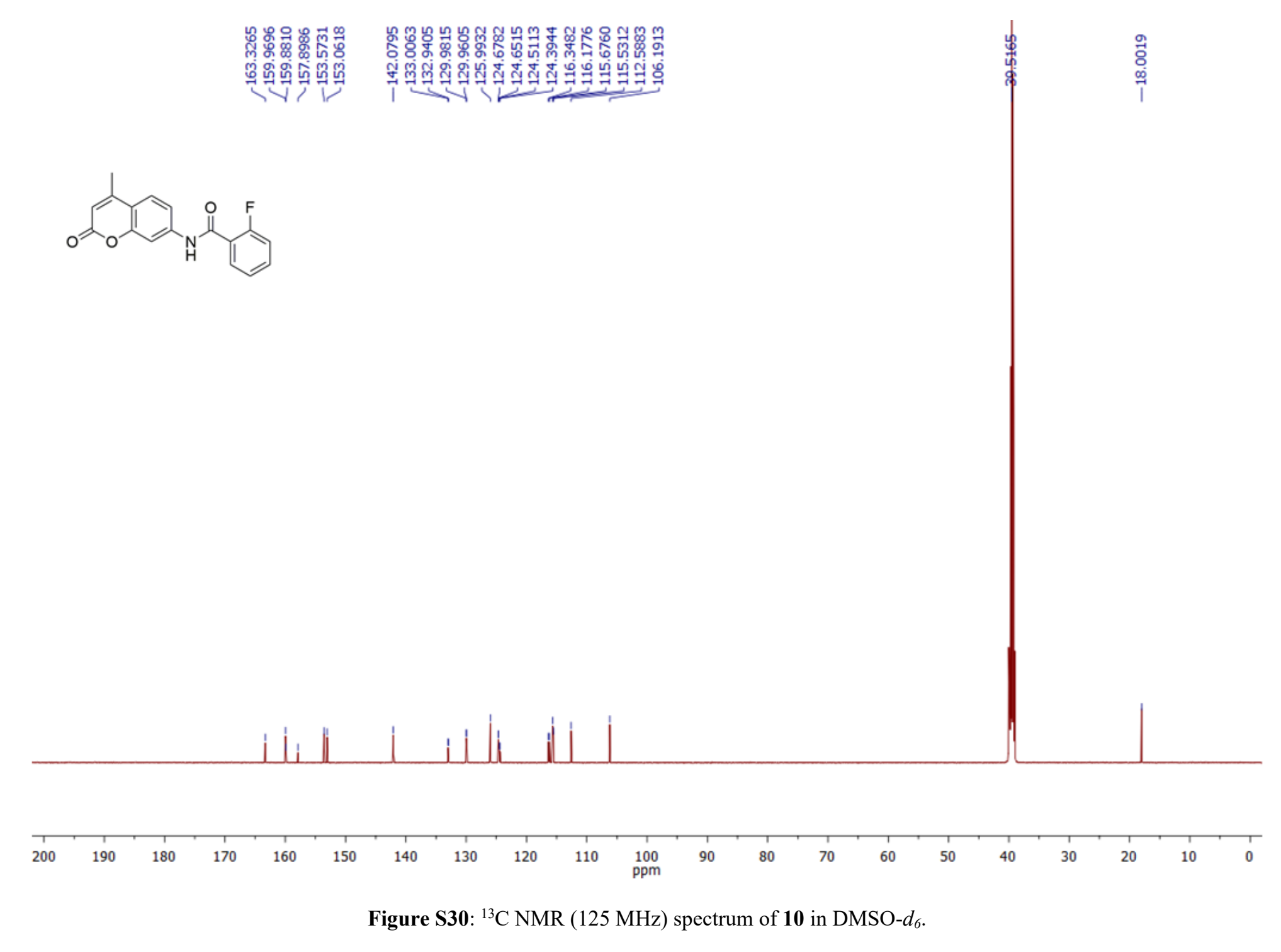


+TOF MS: 0.0925 to 0.0972 min from Sample 4 (SON04-051A) of SON04-051 wiff

Max. $1.5 e 5 \mathrm{cps}$. a=7.026756 14111569260e-004, t0=8.09860493587667320e-001 (DuoSpray ())

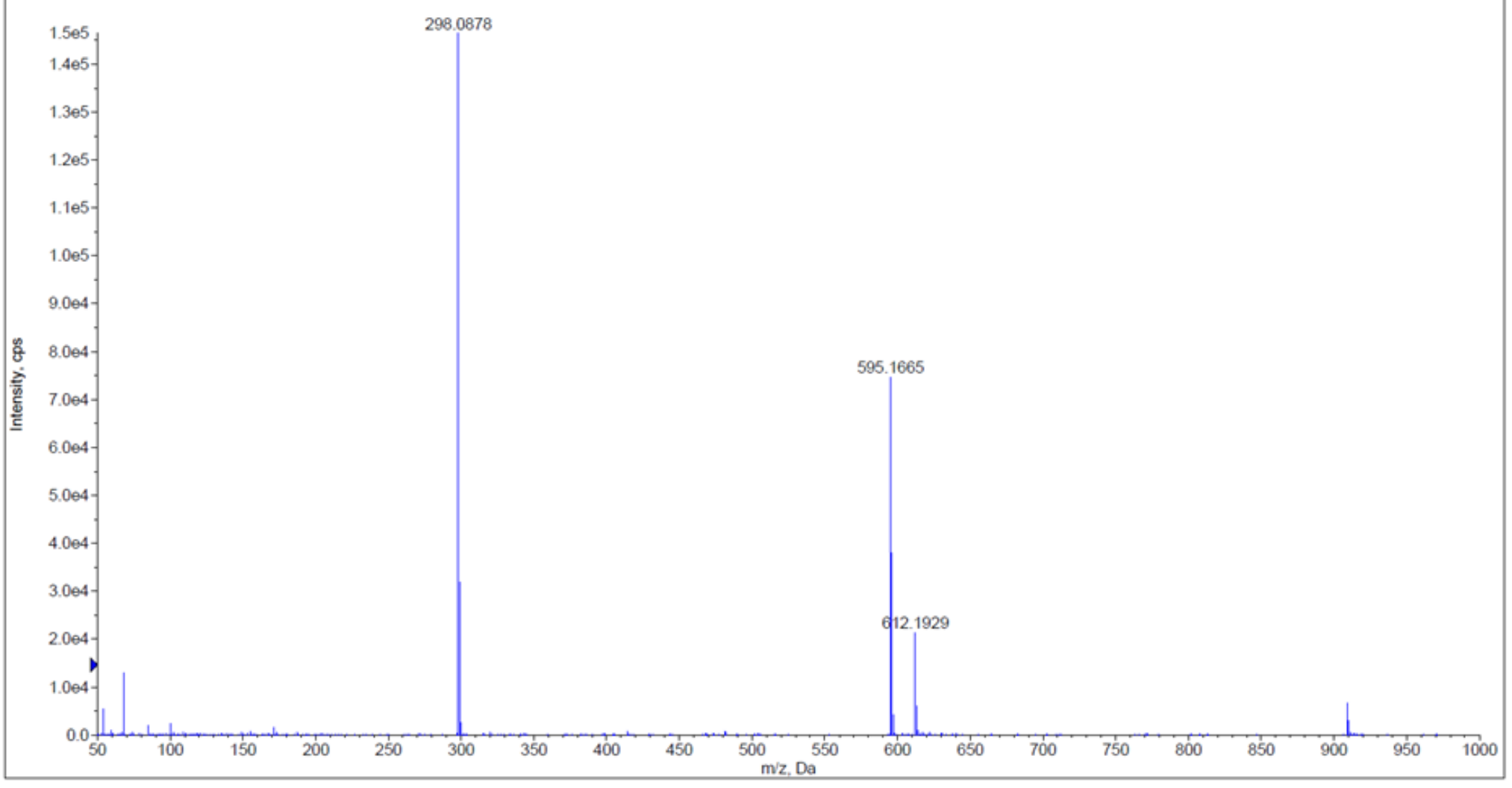

Figure S31: HRMS spectrum for 10. 
Chromatogram

SON04-051A SON04-051A.led

mAU

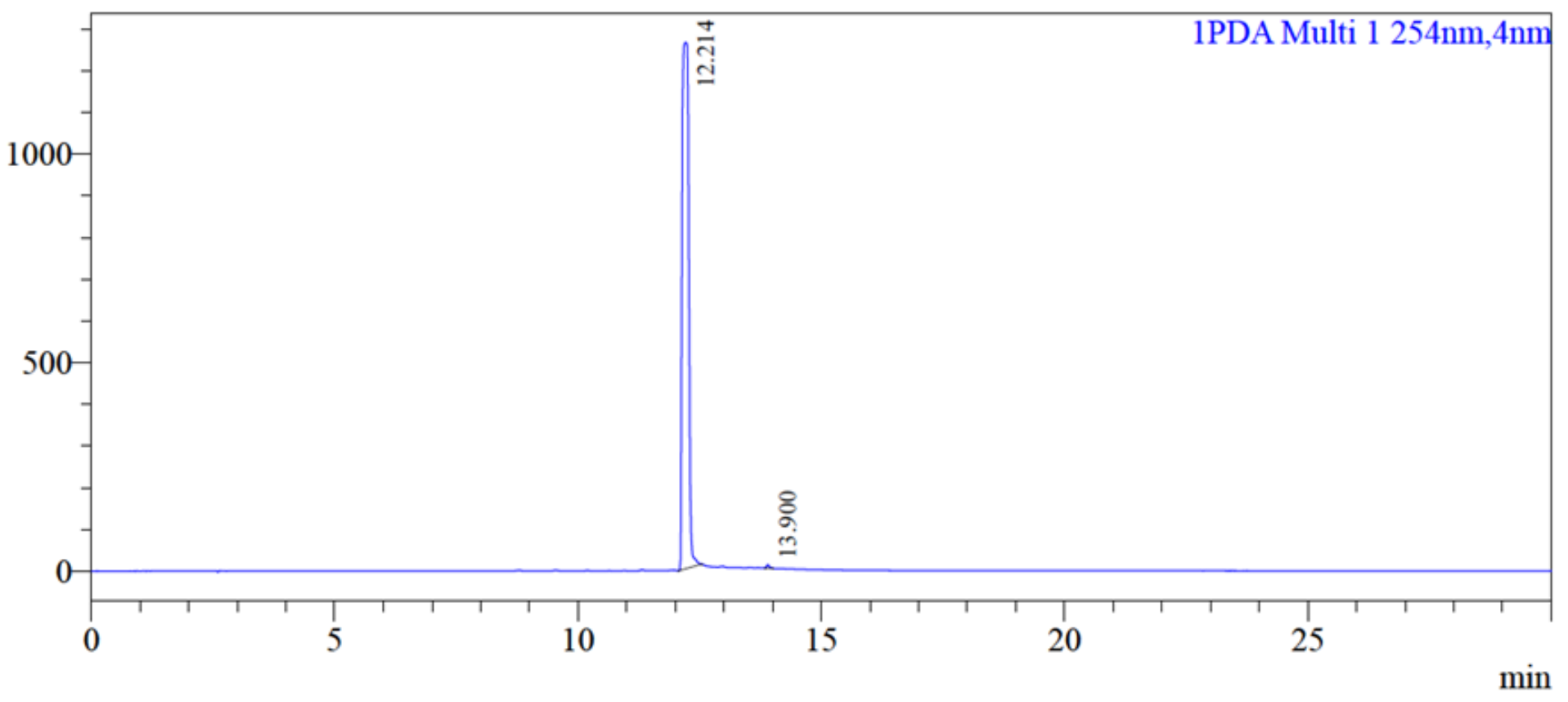

Peak Table

PDA Ch1 $254 \mathrm{~nm}$
\begin{tabular}{|r|r|r|r|r|r|}
\hline Peak\# & Ret. Time & \multicolumn{1}{c|}{ Area } & \multicolumn{1}{c|}{ Height } & Conc. & \\
\hline 1 & 12.214 & 11752408 & 1261283 & 99.741 & \\
\hline 2 & 13.900 & 30575 & 8296 & 0.259 & \\
\hline Total & & 11782983 & 1269579 & & \\
\hline
\end{tabular}

Figure S32: RP-HPLC trace for $\mathbf{1 0 .}$ 


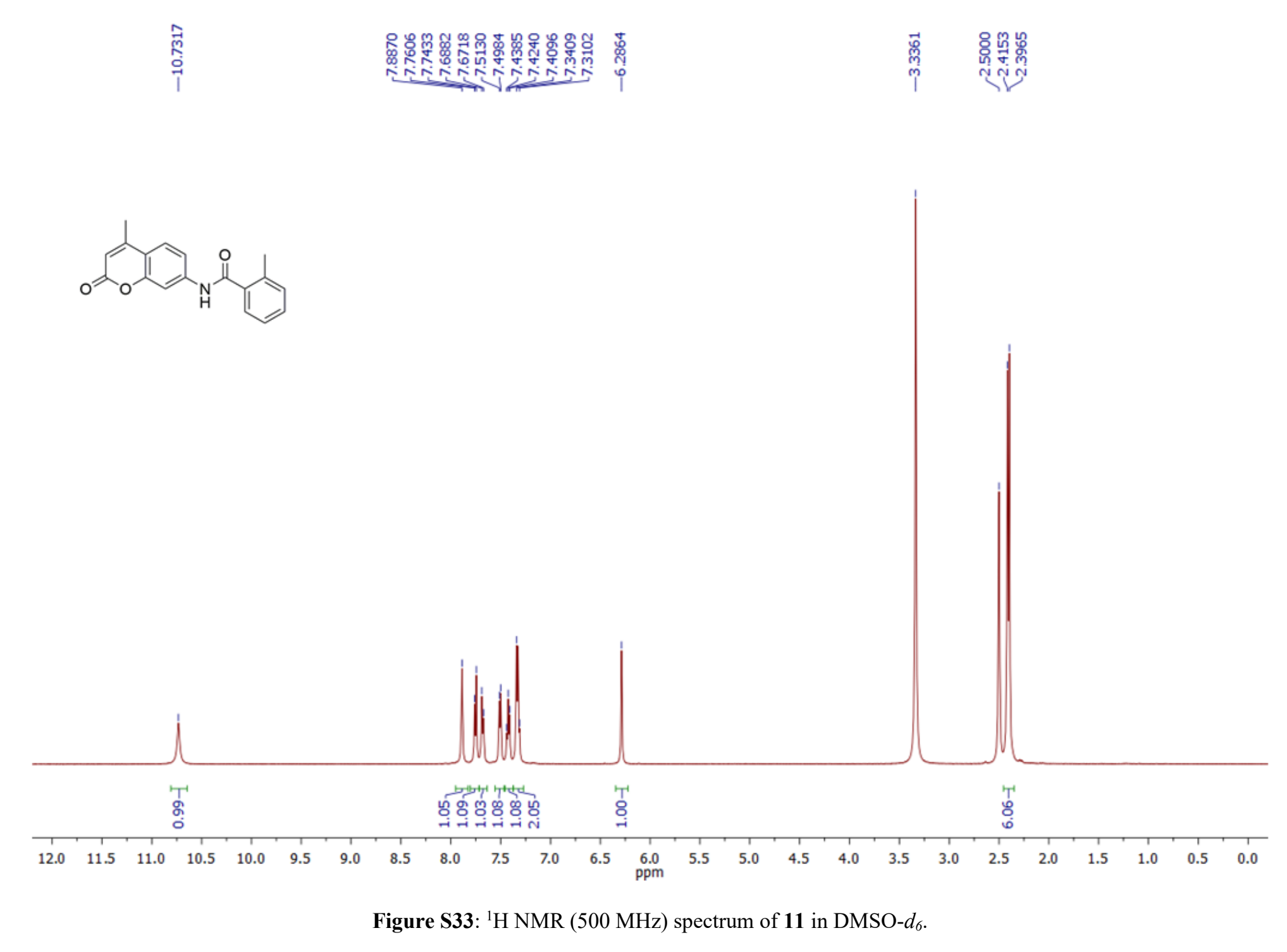




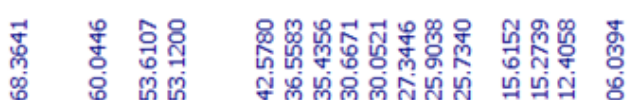

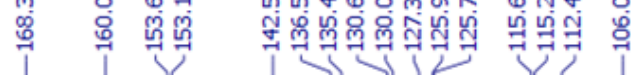
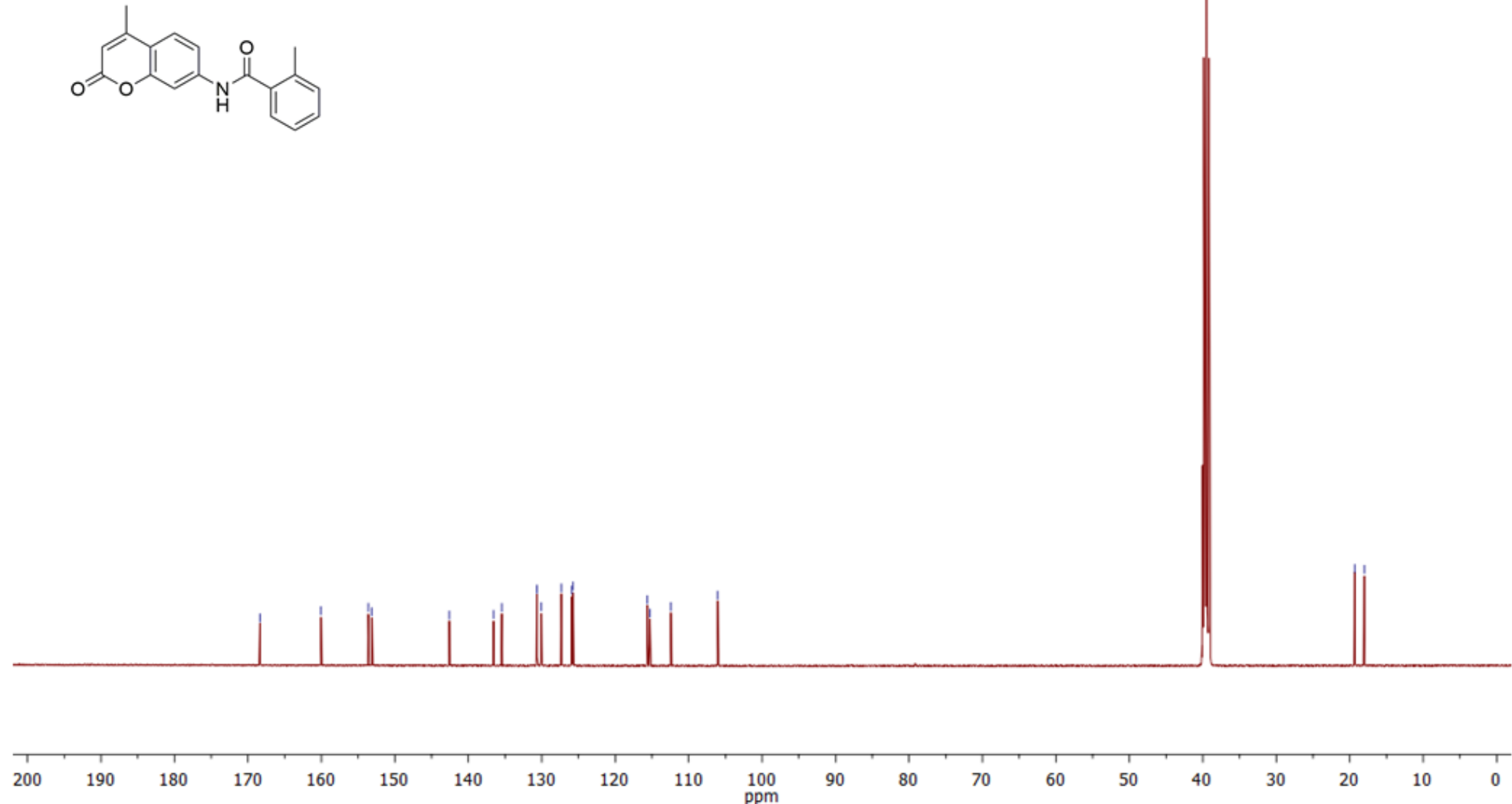

Figure S34: ${ }^{13} \mathrm{C}$ NMR $(125 \mathrm{MHz})$ spectrum of 11 in DMSO- $d_{6}$ 


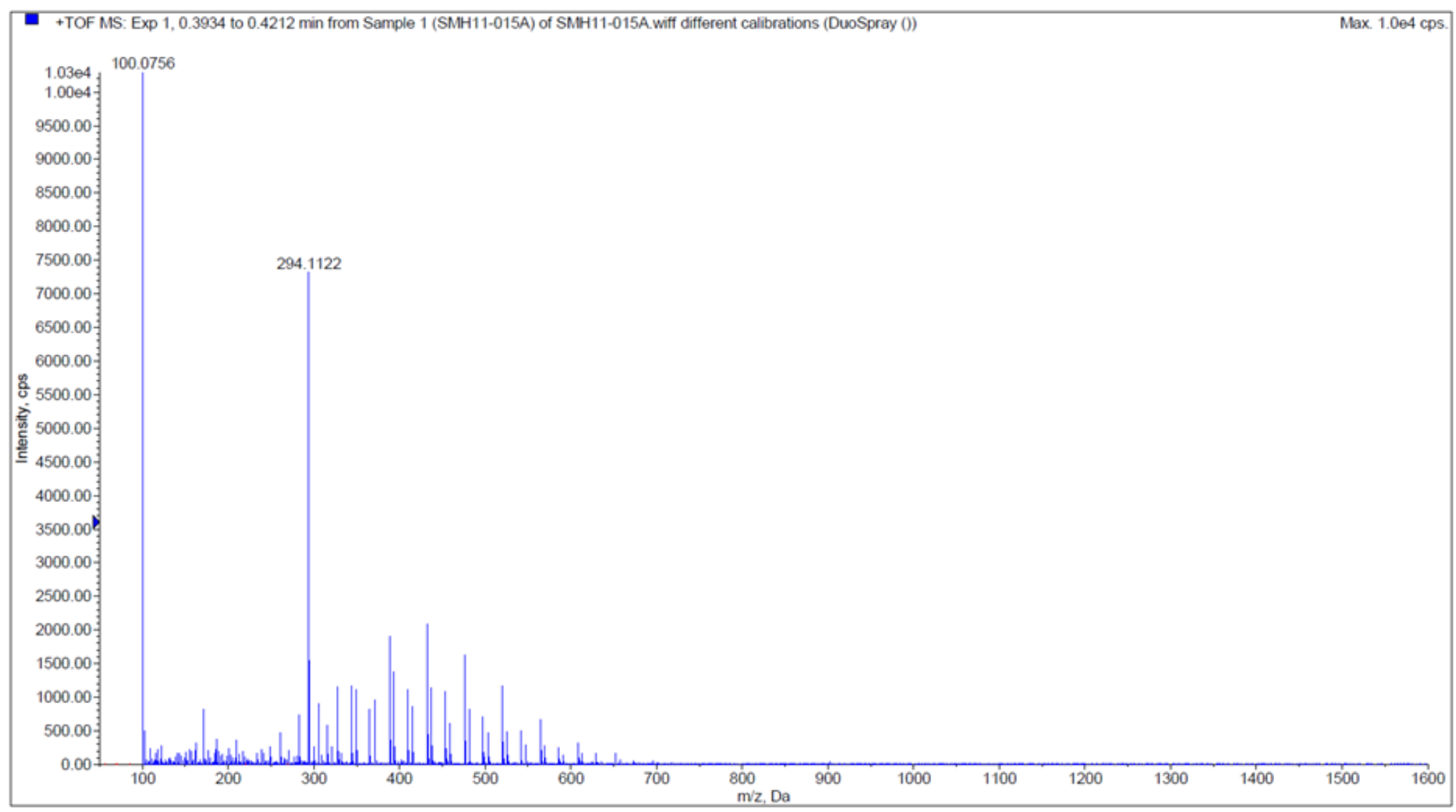

Figure S35: HRMS spectrum of 11. 
Chromatogram

mAU

SMH11-015A SMH11-015A.lcd

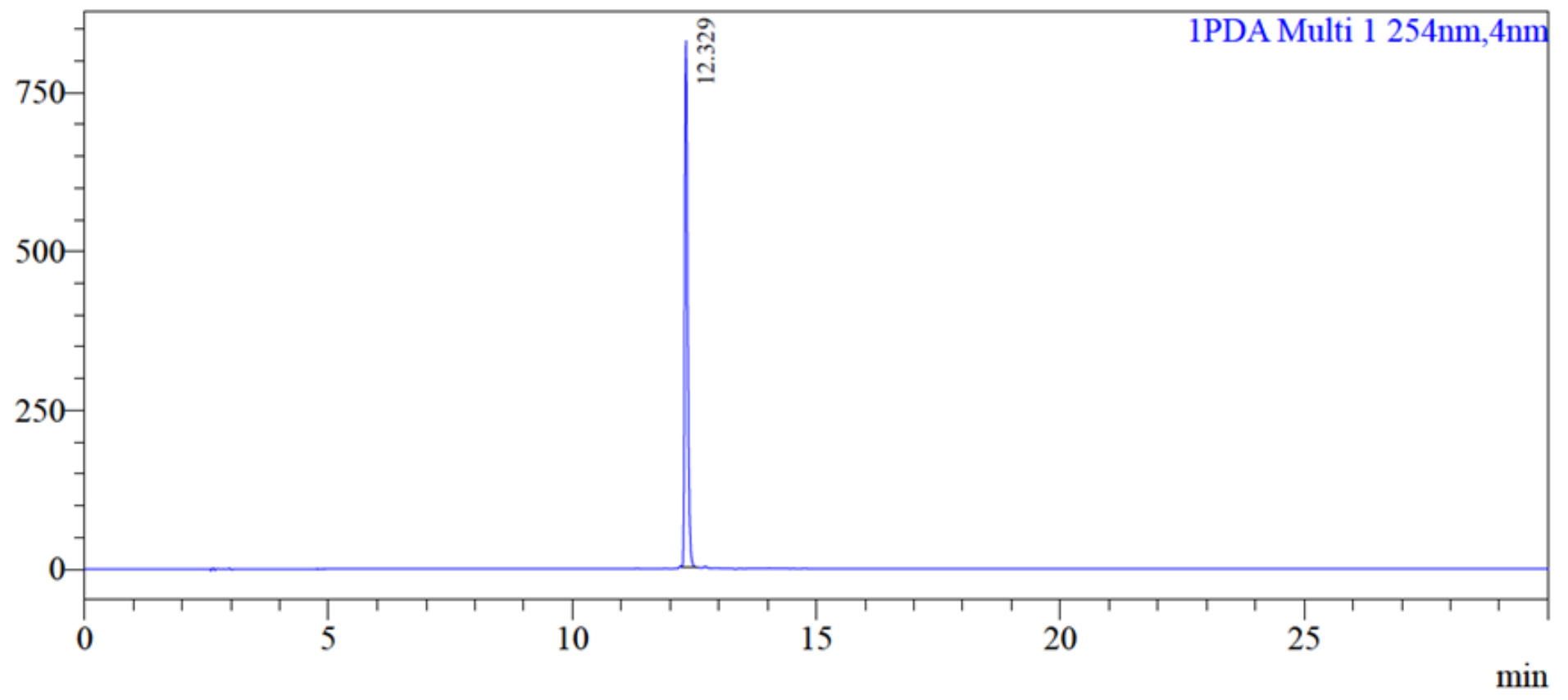

Peak Table

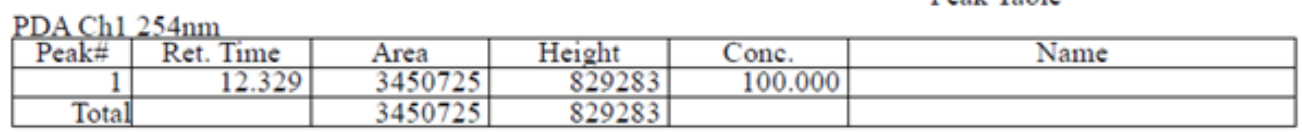

Figure S36: RP-HPLC trace of 11. 

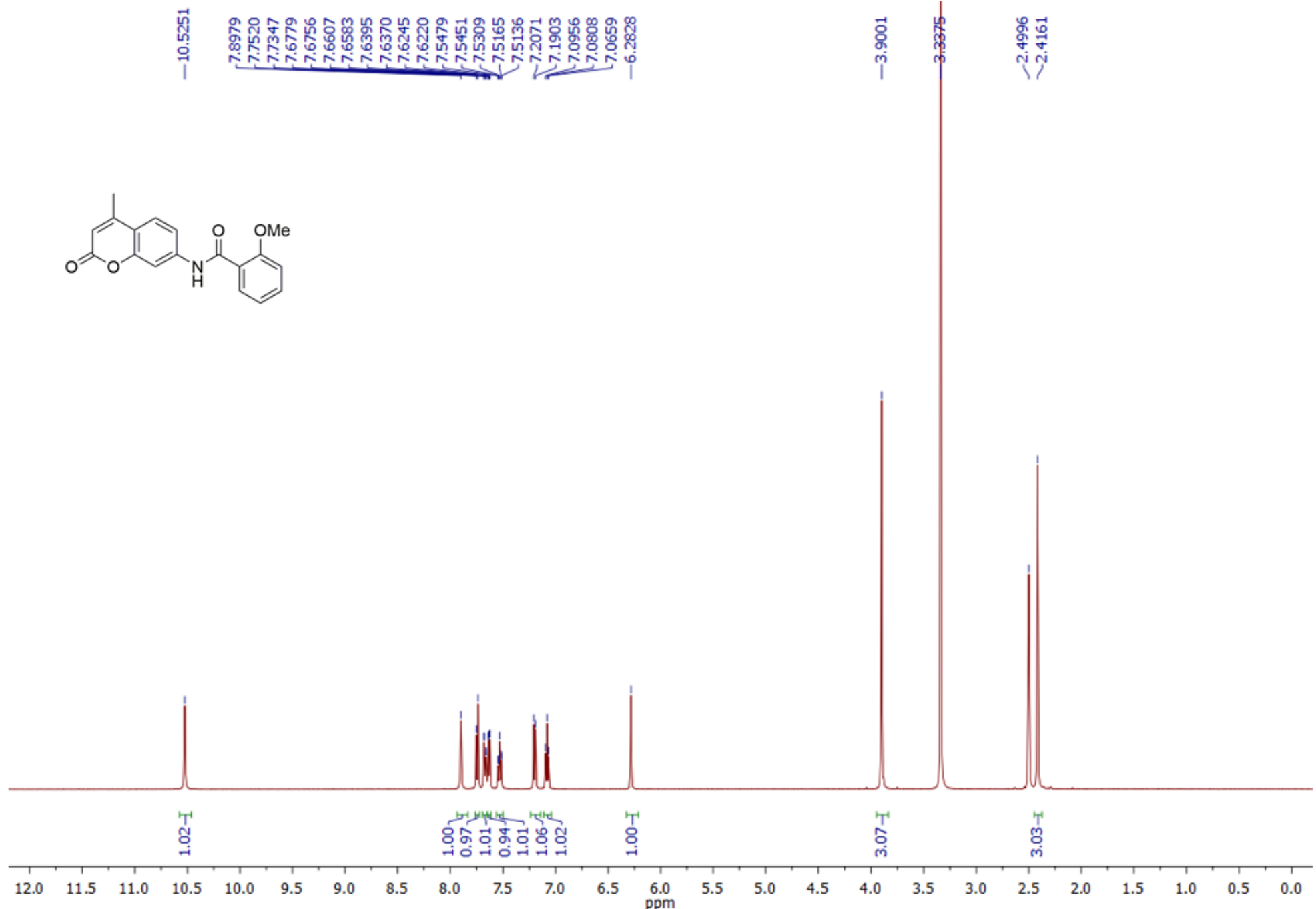

Figure S37: ${ }^{1} \mathrm{H}$ NMR $(500 \mathrm{MHz})$ spectrum of 12 in DMSO- $d_{6}$. 


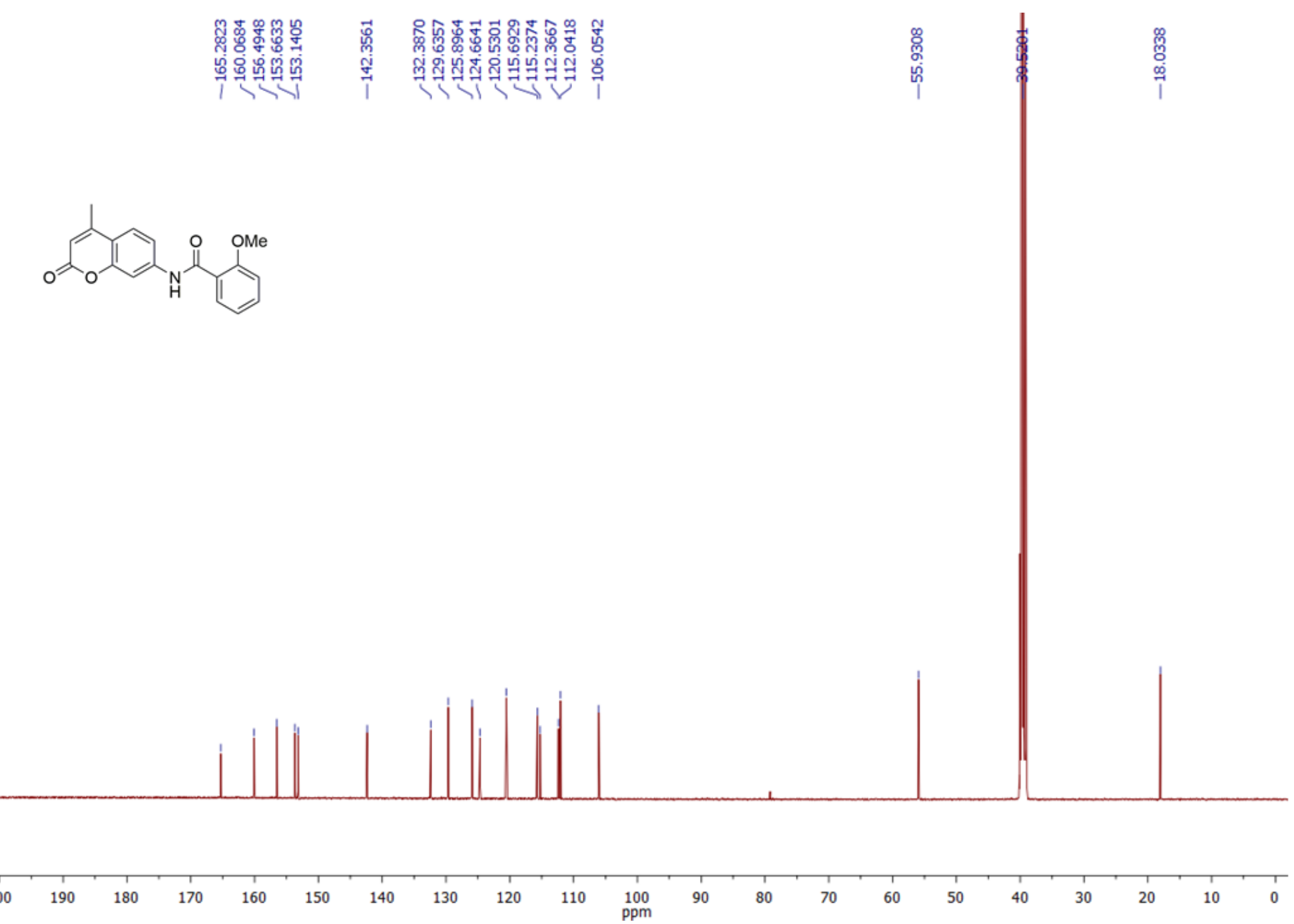

Figure S38: ${ }^{13} \mathrm{C}$ NMR (125 MHz) spectrum of 12 in DMSO- $d_{6}$. 
- +TOF MS: Exp 1, 0.4953 to 0.5231 min from Sample 1(SMH11-051A) of SMH11-051A wiff different calibrations (DuoSpray ())

Max. $27 \mathrm{e} 4 \mathrm{cps}$.

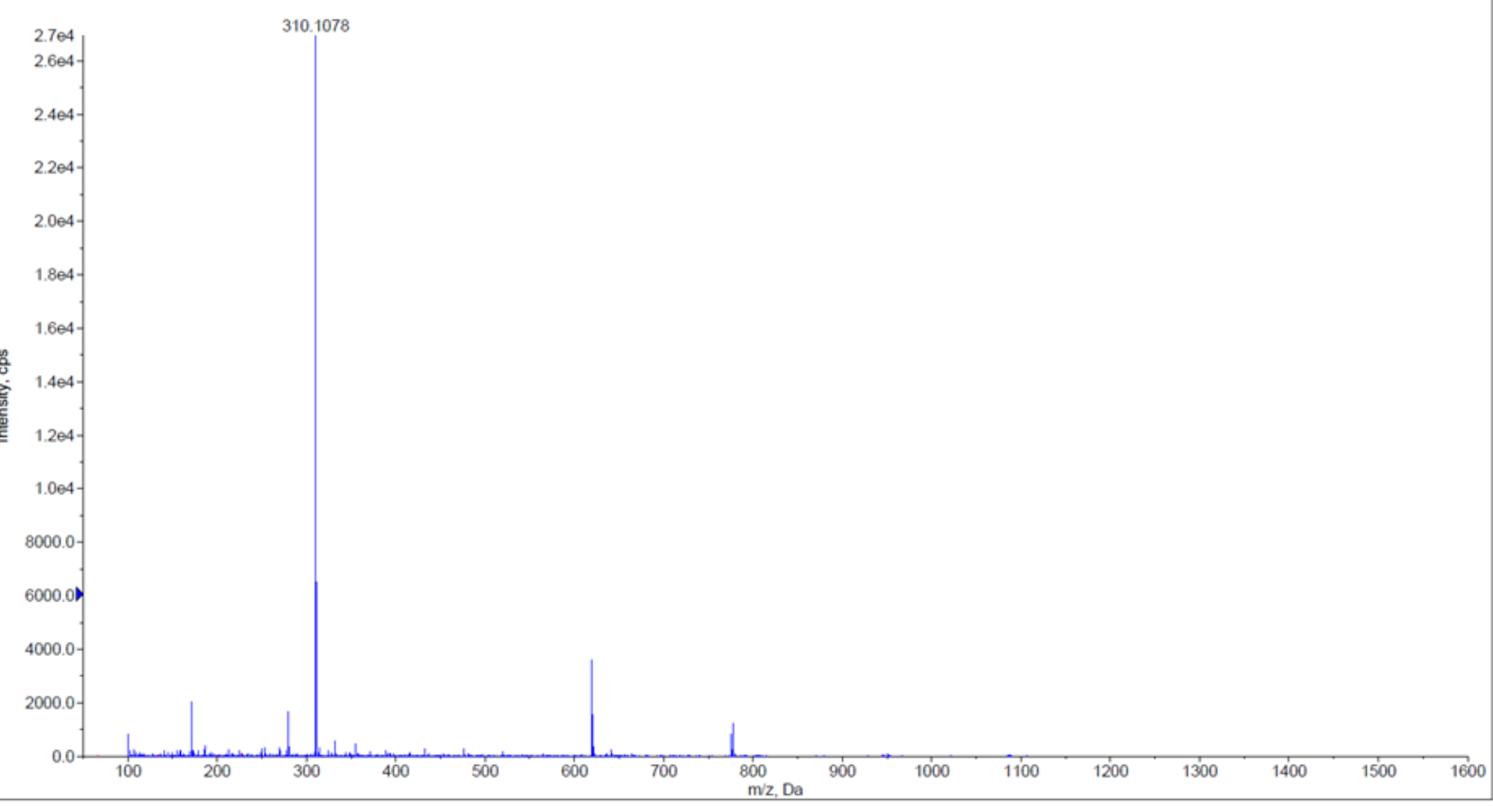

Figure S39: HRMS spectrum of 12 
Chromatogram

mAU

SMH11-051A SMH11-051A.lcd

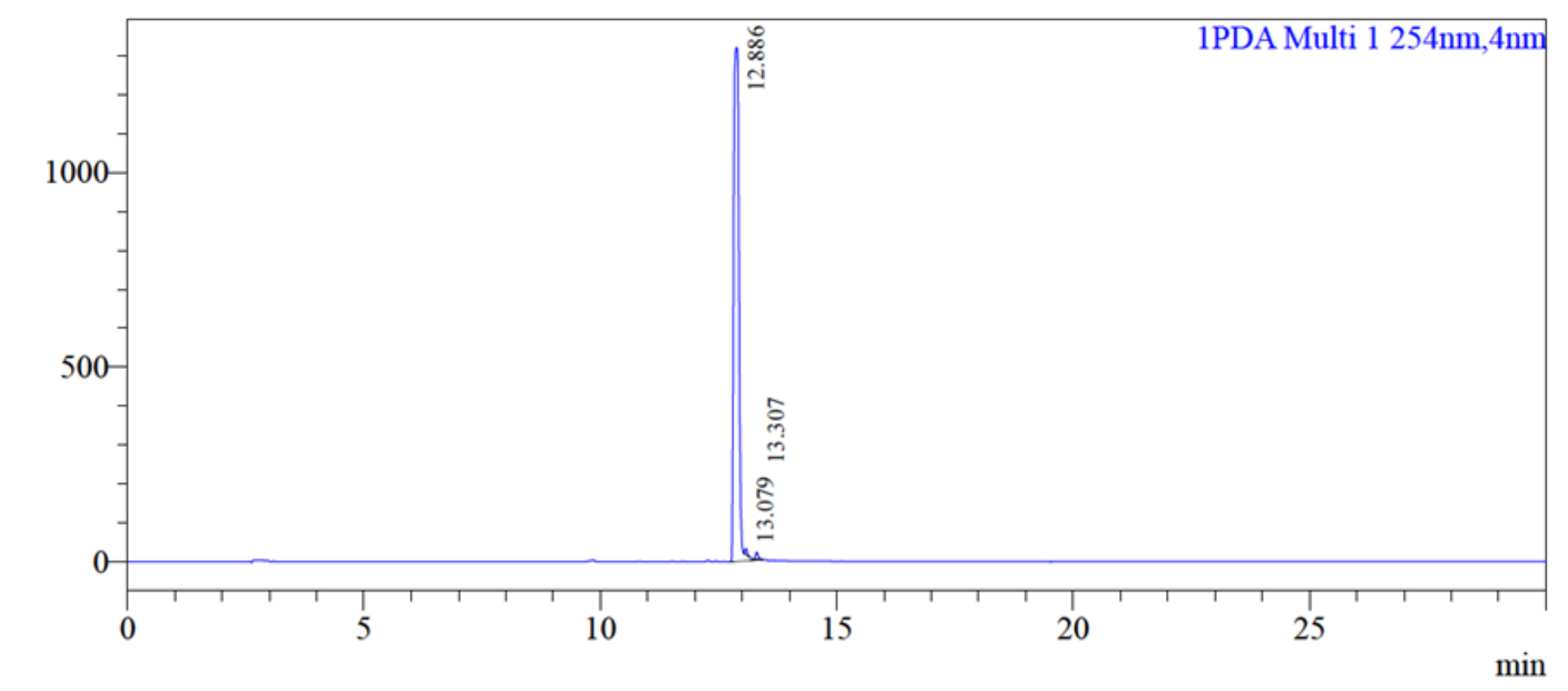

Peak Table

PDA Ch1 $254 \mathrm{~nm}$
\begin{tabular}{|r|r|r|r|r|l|}
\hline Peak\# & Ret. Time & \multicolumn{1}{|c|}{ Area } & \multicolumn{1}{|c|}{ Height } & Conc. & \\
\hline 1 & 12.886 & 10200023 & 1318793 & 98.849 & \\
\hline 2 & 13.079 & 48310 & 15751 & 0.468 & \\
\hline 3 & 13.307 & 70461 & 18635 & 0.683 & \\
\hline Total & & 10318794 & 1353179 & & \\
\hline
\end{tabular}

Figure S40: RP-HPLC trace of 12. 


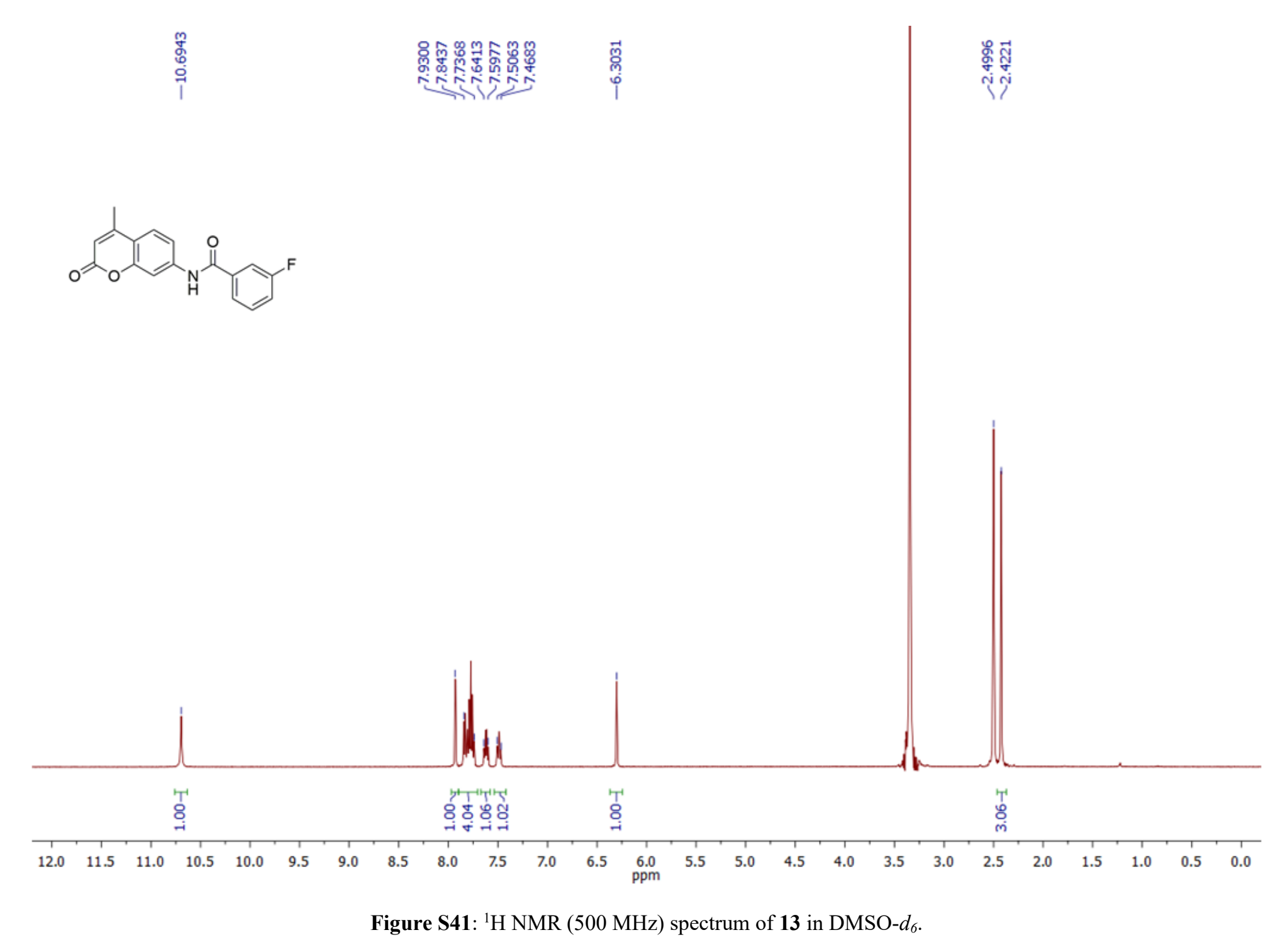




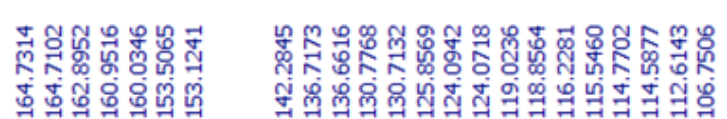

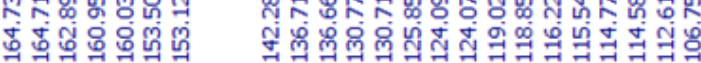
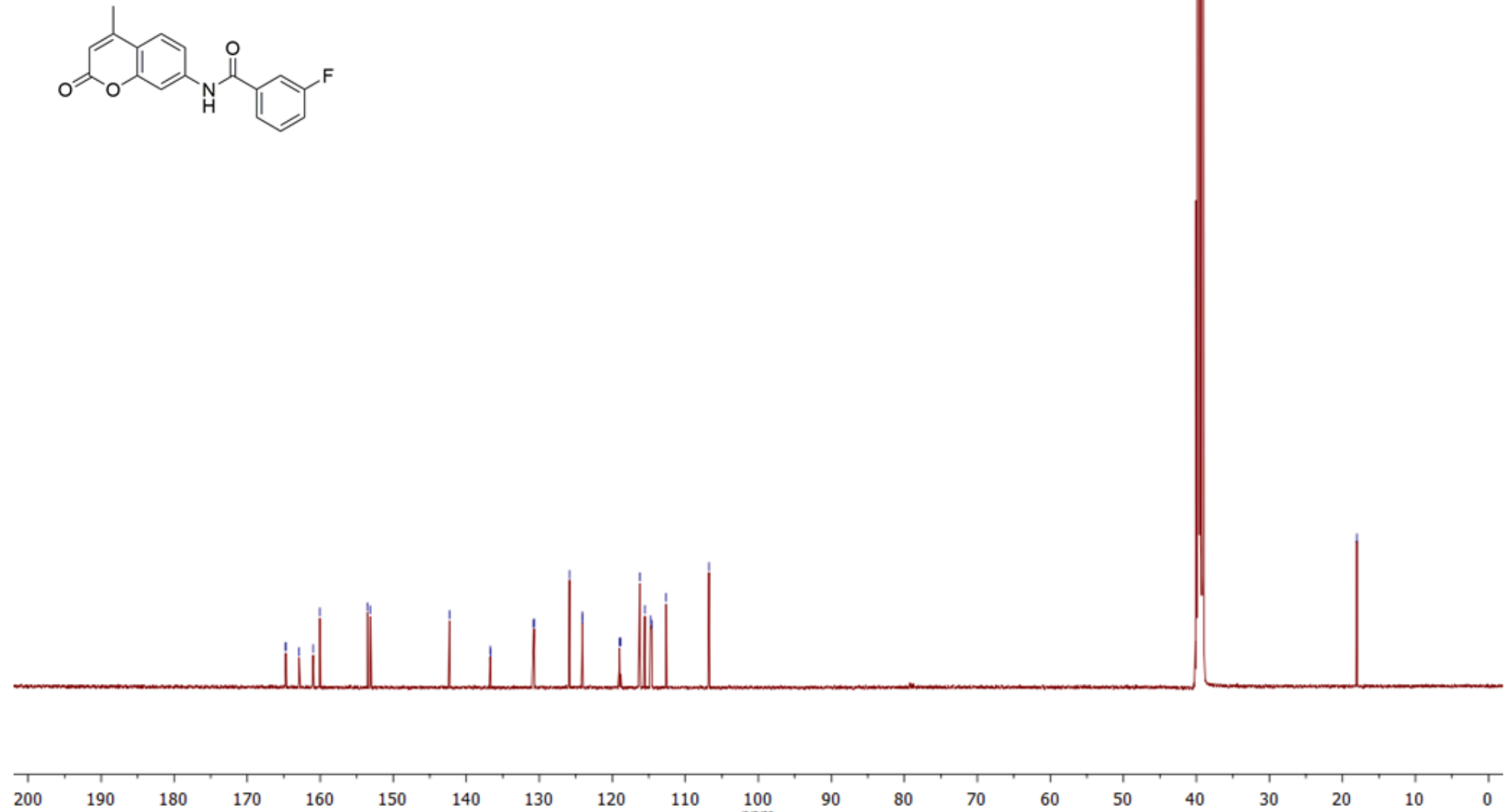

Figure S42: ${ }^{13} \mathrm{C}$ NMR $(125 \mathrm{MHz})$ spectrum of 13 in DMSO- $d_{6}$ 
+TOF MS: Exp 1, 0.1154 to 0.1247 min from Sample 1(SMH11-067A) of SMH11-067A.wiff

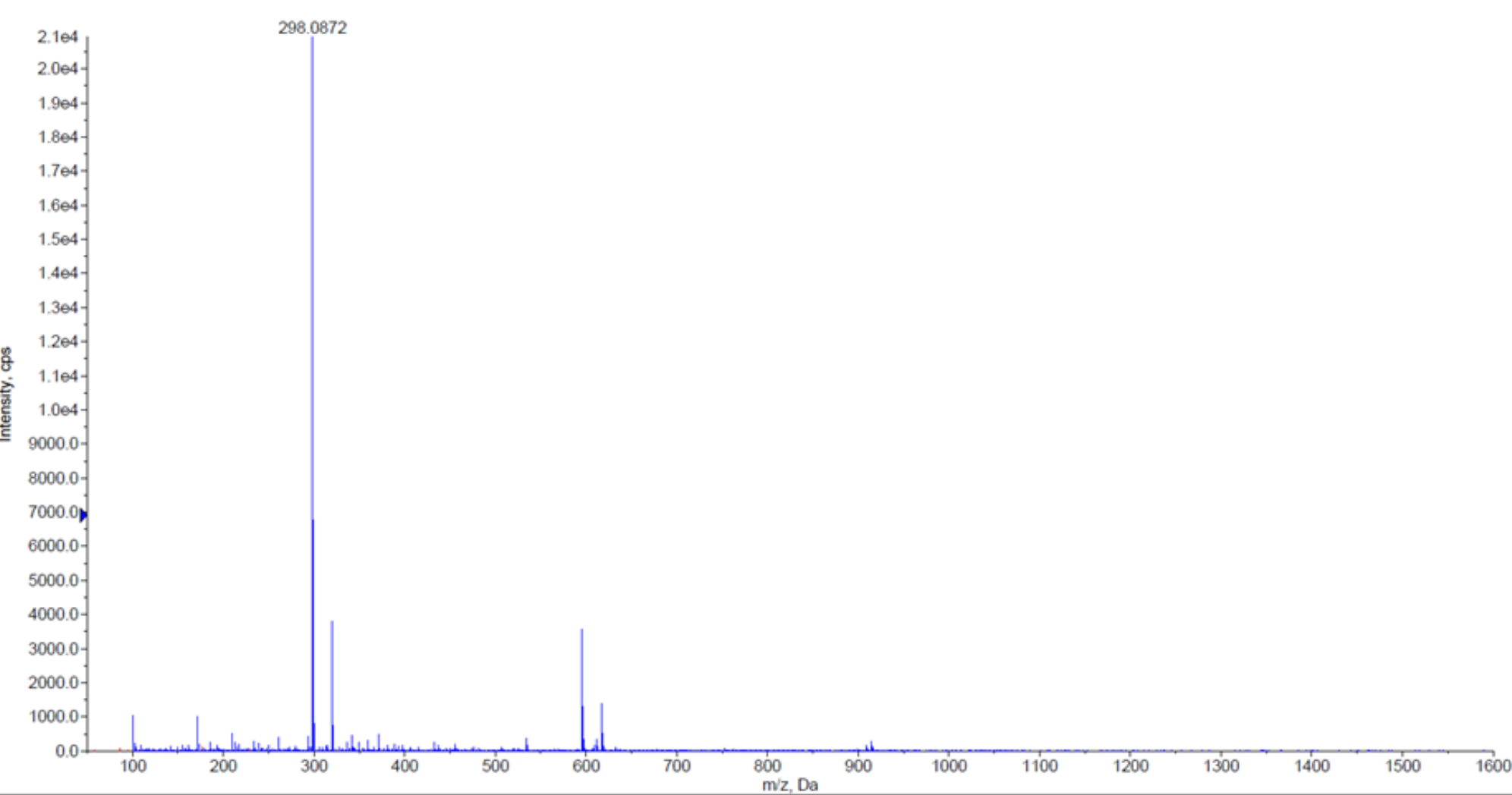

Figure S43: HRMS spectrum of 13. 
Chromatogram

SMH11-067A SMH11-067A.led

mAU

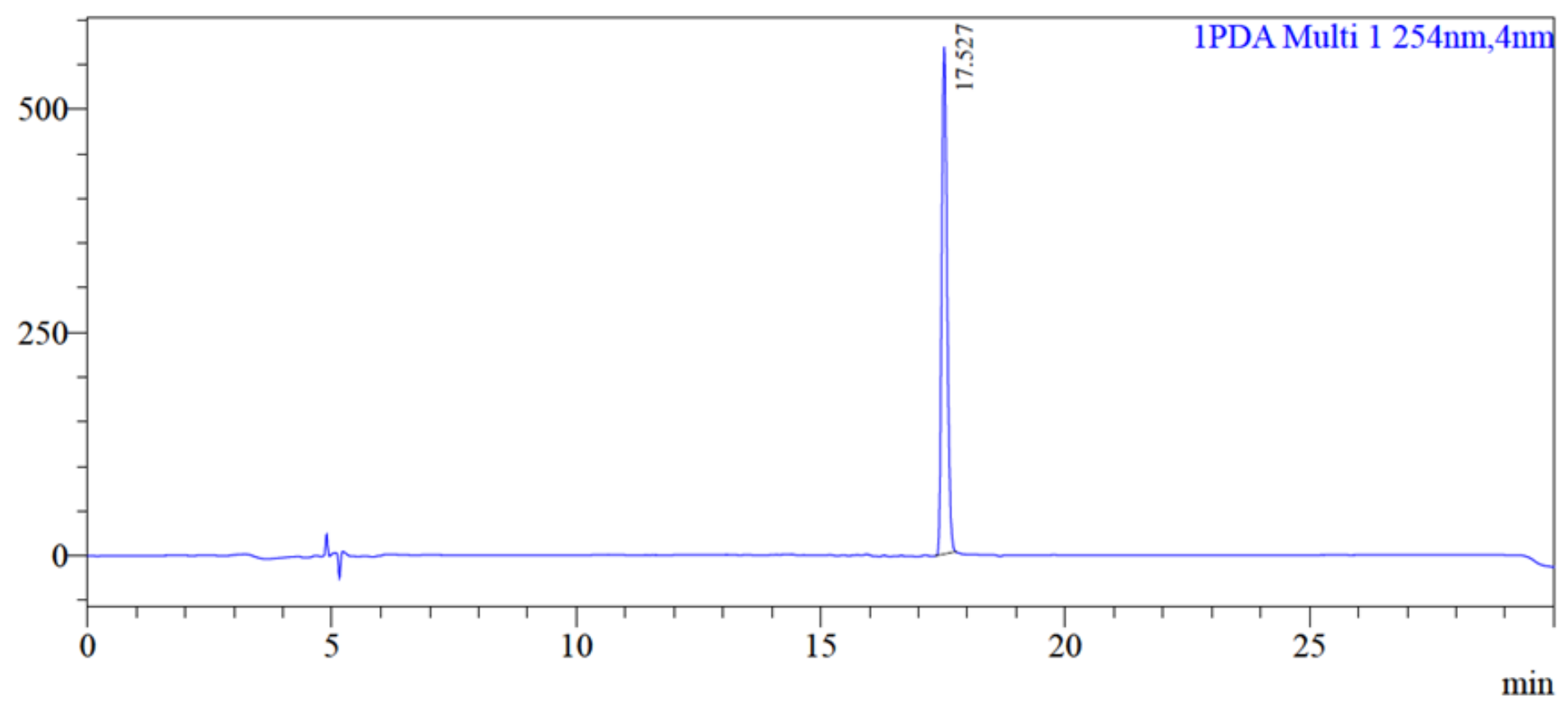

Peak Table

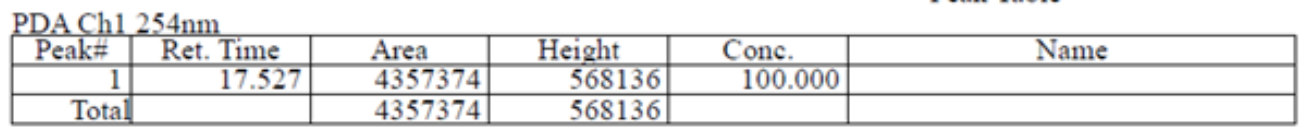

Figure S44: RP-HPLC trace of 13. 


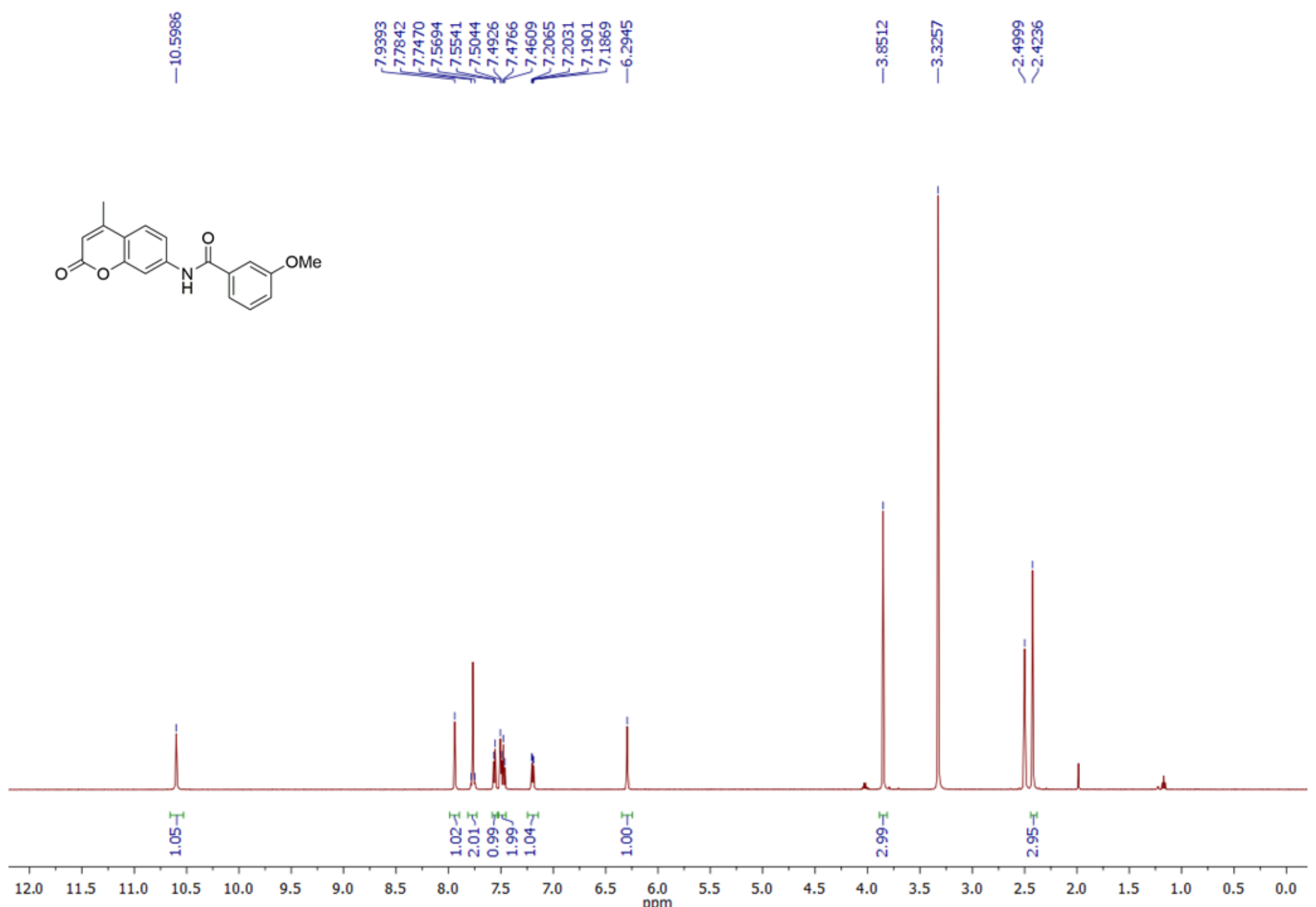

Figure S45: ${ }^{1} \mathrm{H}$ NMR $(500 \mathrm{MHz})$ spectrum of 14 in DMSO- $d_{6}$. 


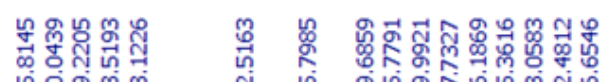

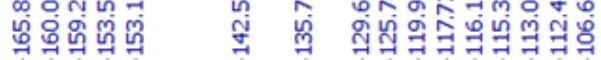

रो $\overrightarrow{1}$ ।
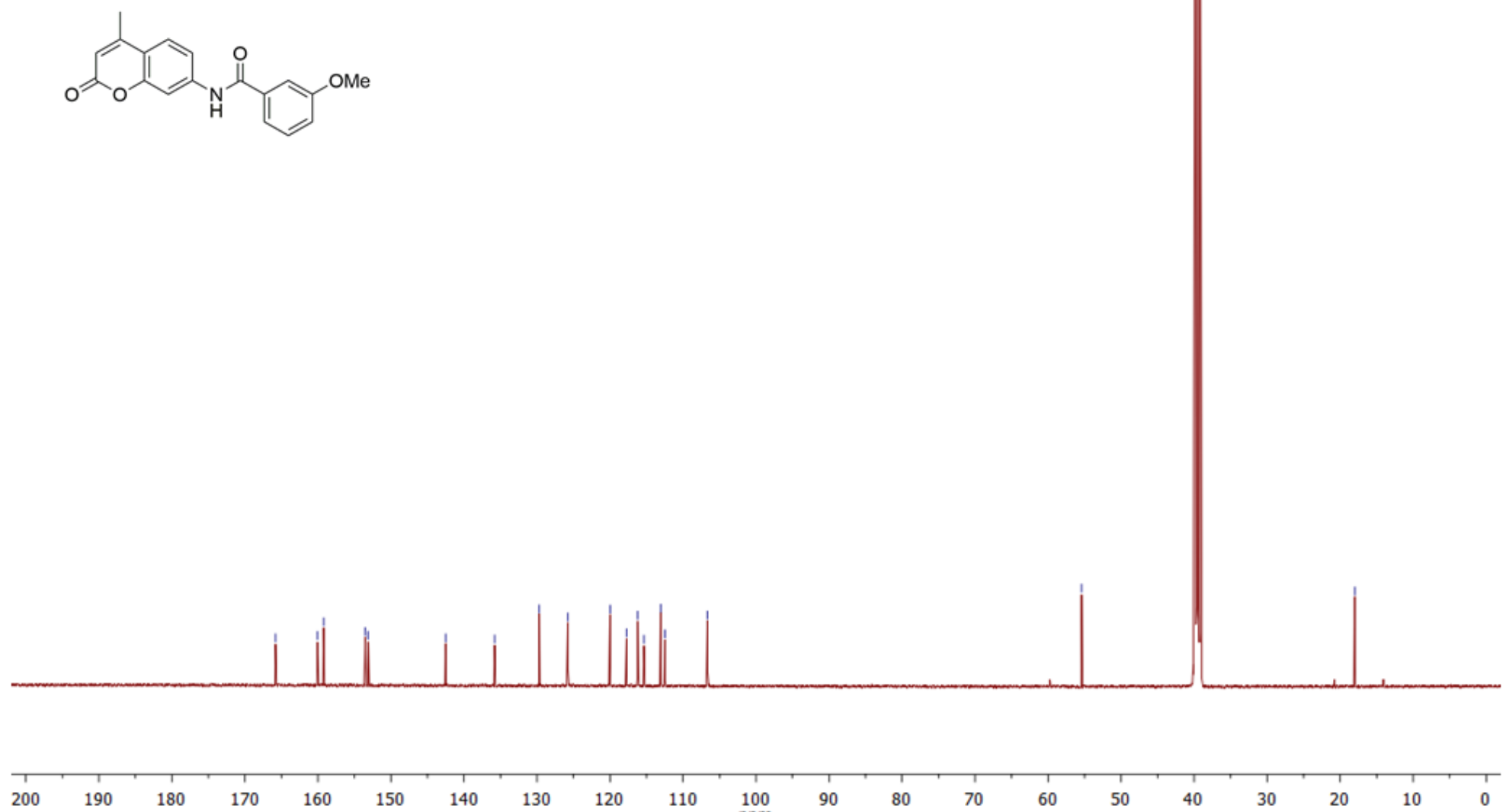

Figure S46: ${ }^{13} \mathrm{C}$ NMR (125 MHz) spectrum of 14 in DMSO- $d_{6}$ 
+TOF MS: 0.1670 to $0.1763 \mathrm{~min}$ from Sample 2 (SMH12-095C) of SMH12-095C. wift

$\mathrm{a}=7.02675052068374500 \mathrm{e}-004,0=7.95711878470257480 \mathrm{e}-001$ (DuoSpray ())

Max $1.6 \mathrm{e} 5 \mathrm{cps}$.

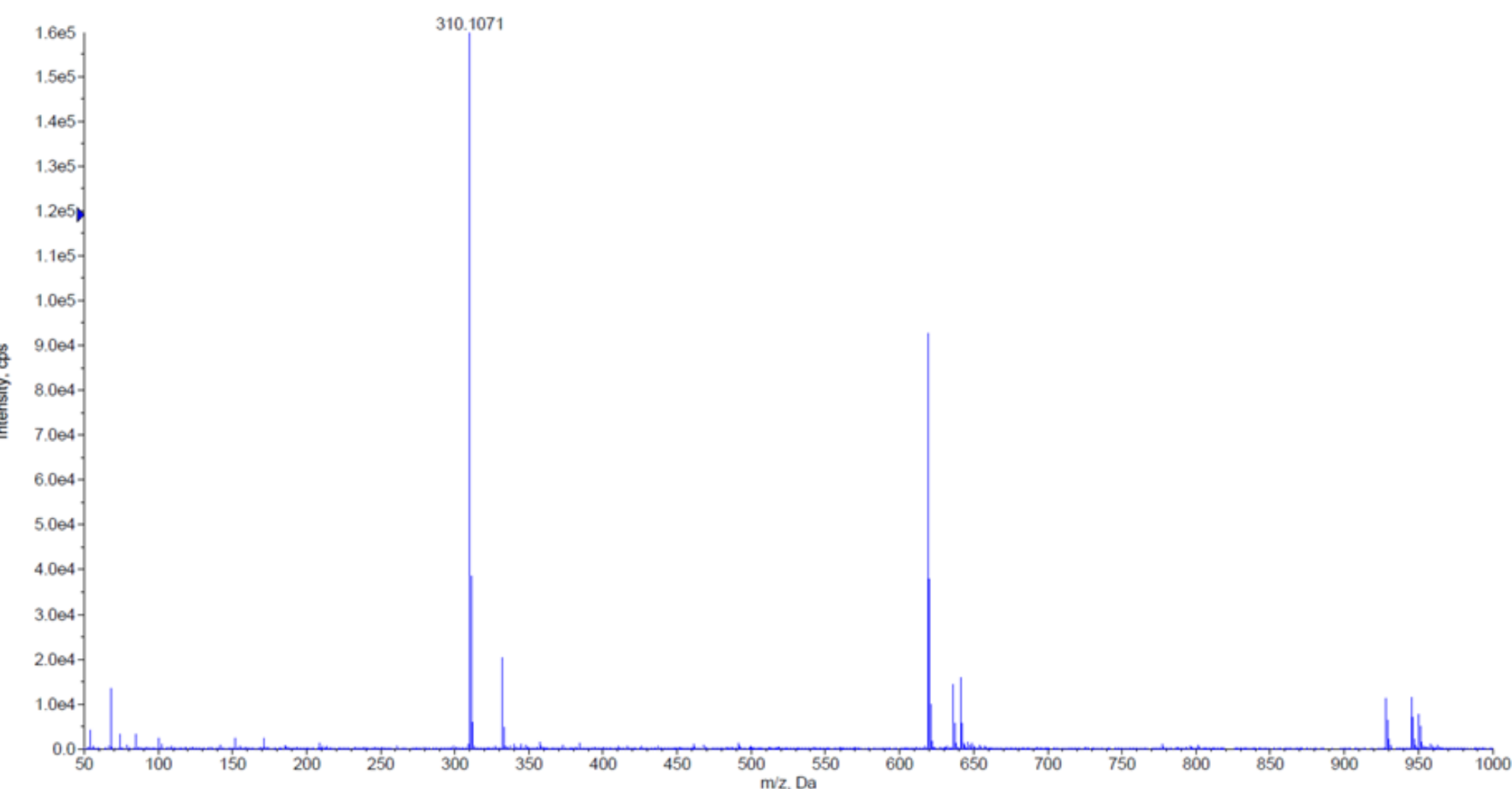

Figure S47: HRMS spectrum of $\mathbf{1 4}$ 
Chromatogram

mAU

SMH12-095C SMH12-095C.lcd

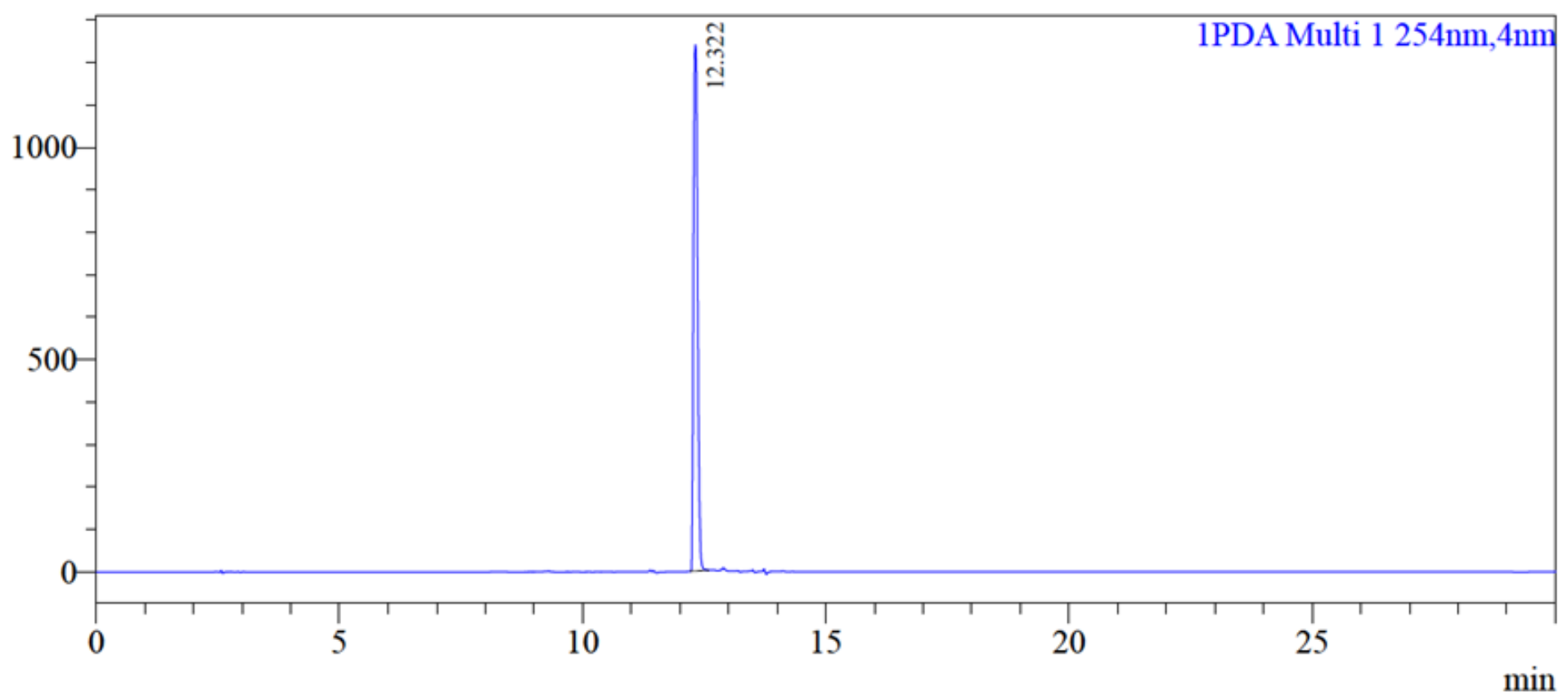

Peak Table

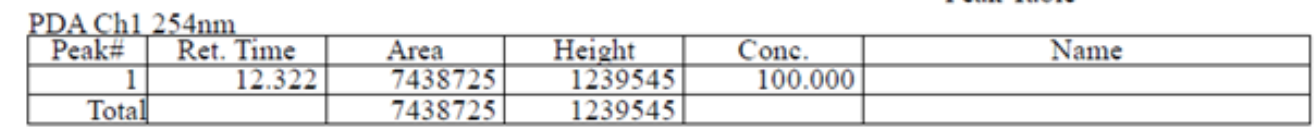

Figure S48: RP-HPLC trace of 14. 


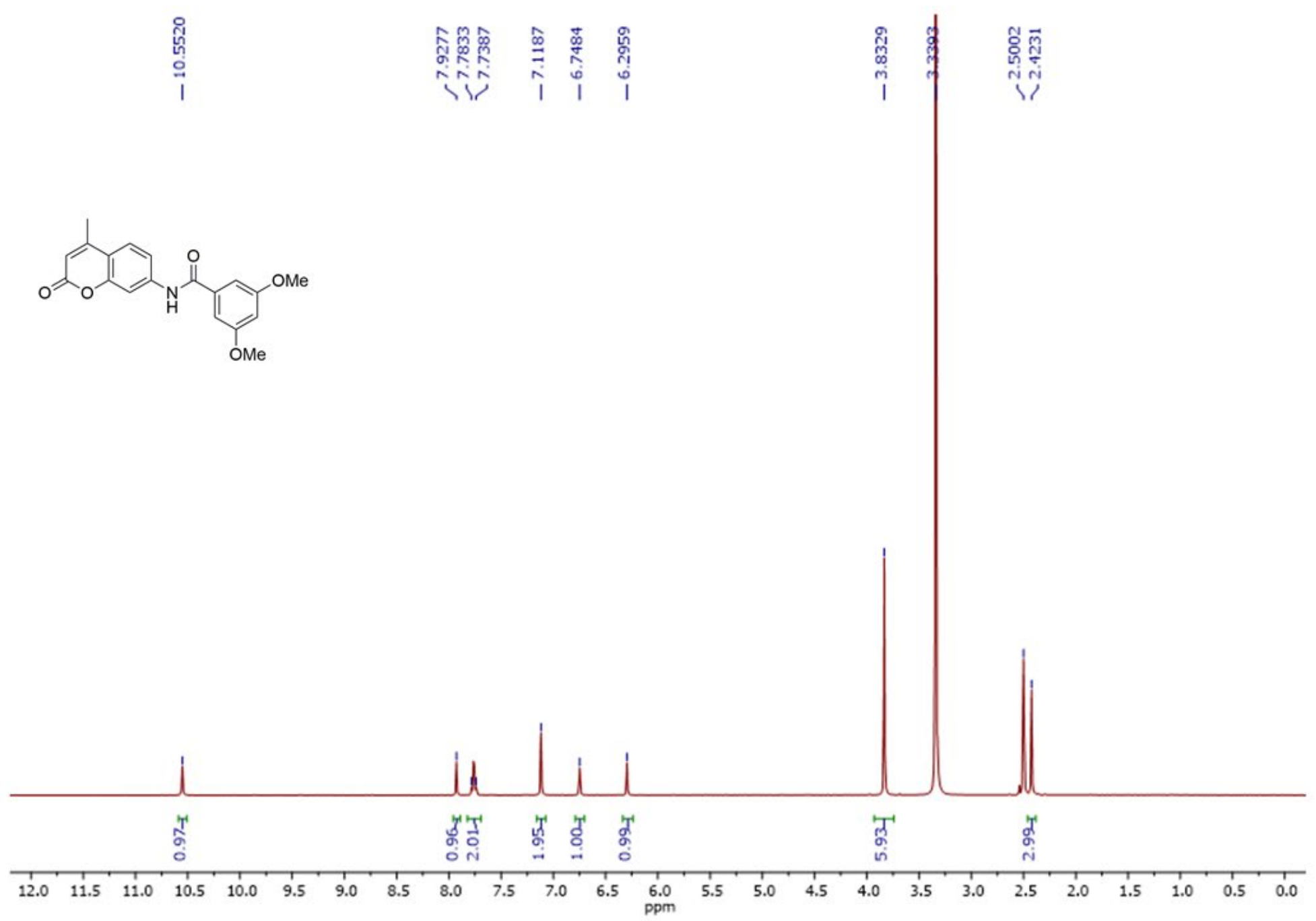

Figure S49: ${ }^{1} \mathrm{H}$ NMR $(500 \mathrm{MHz})$ spectrum of 15 in DMSO- $d_{6}$. 

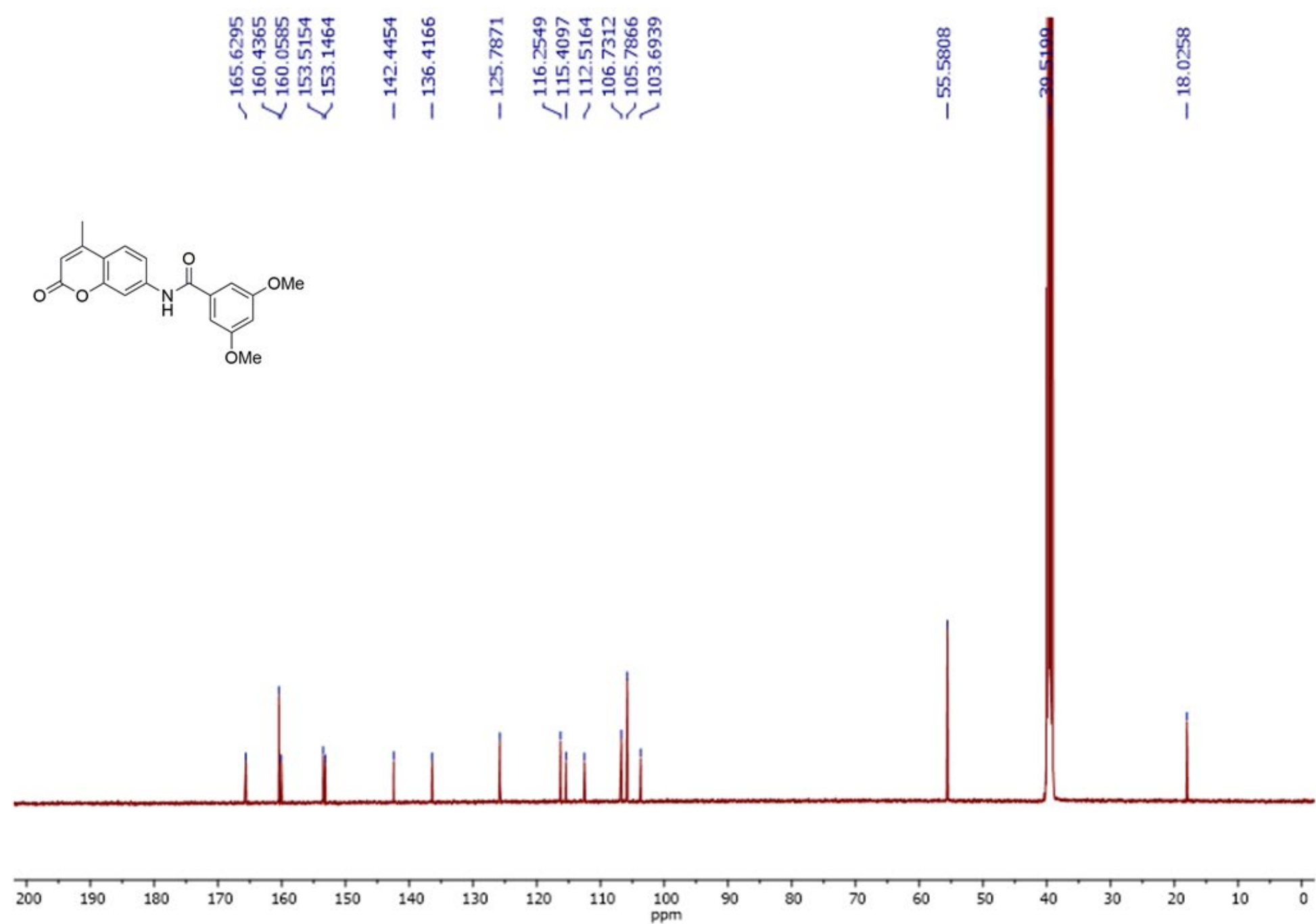

Figure S50: ${ }^{13} \mathrm{C}$ NMR $(500 \mathrm{MHz})$ spectrum of 15 in DMSO- $d_{6}$ 


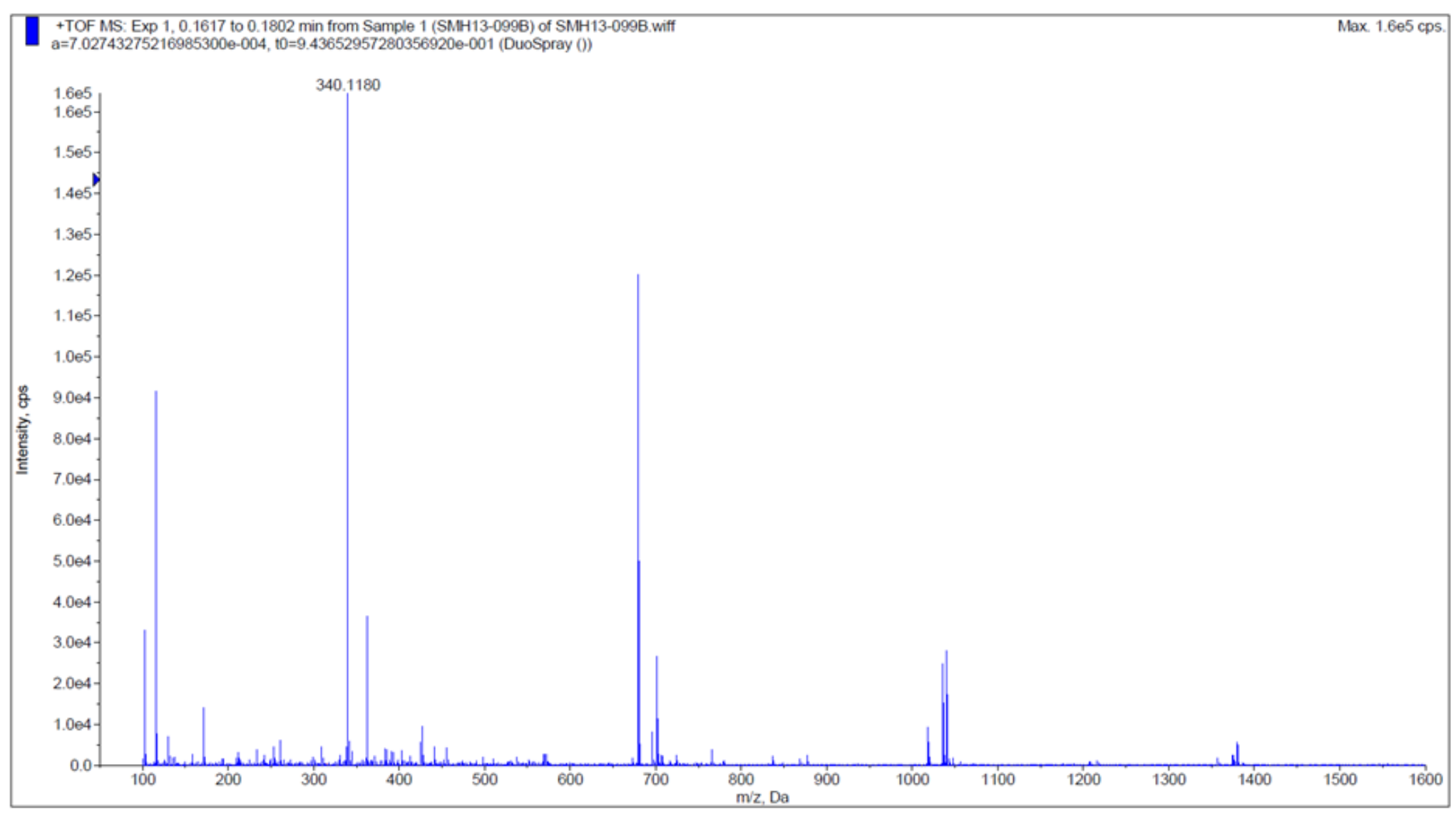

Figure S51: HRMS spectrum for 15. 
Chromatogram

SMH13-099B SMH13-099B.lcd

mAU

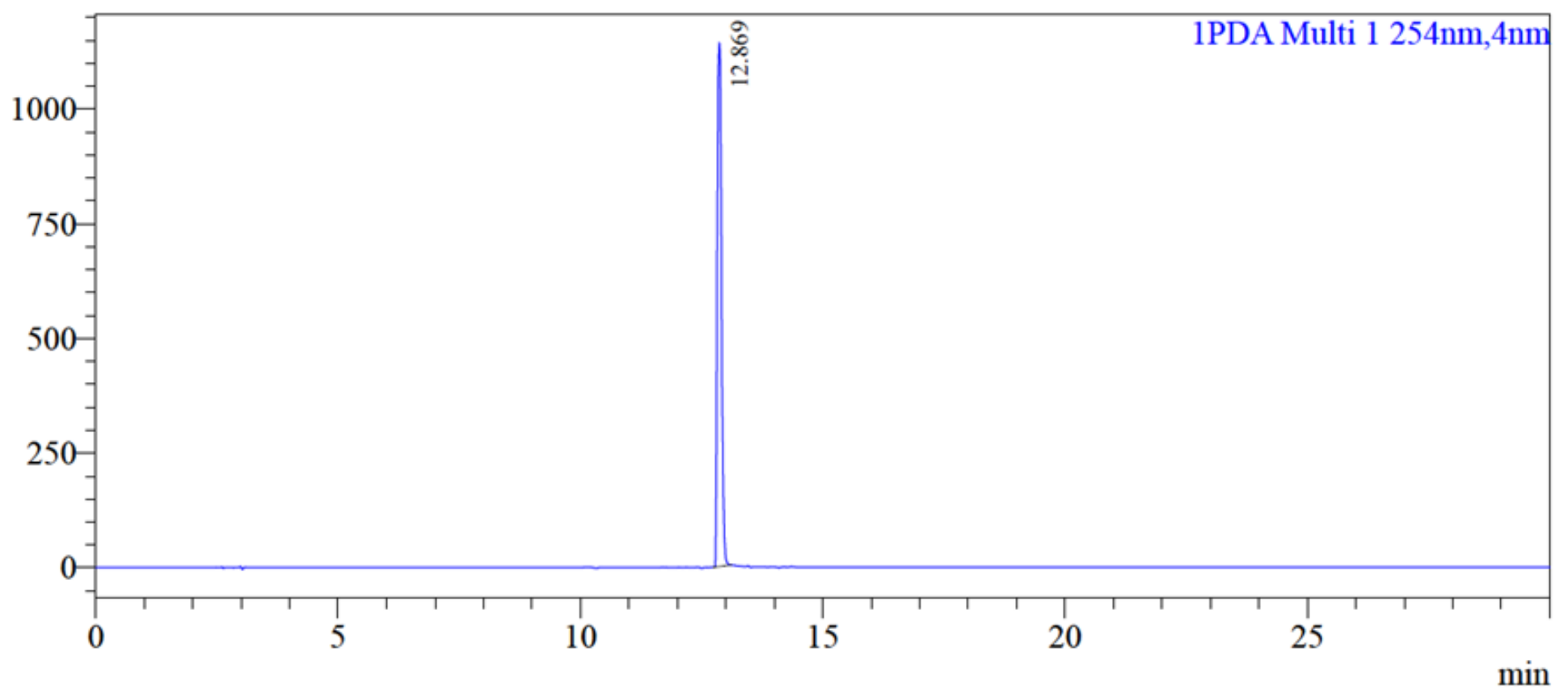

Peak Table

PDA Ch1 $254 \mathrm{~nm}$
\begin{tabular}{|r|r|r|r|r|r|}
\hline Peak $\#$ & Ret. Time & Area & Height & Conc. & Name \\
\hline 1 & 12.869 & 6965575 & 1141278 & 100.000 & \\
\hline Total & & 6965575 & 1141278 & & \\
\hline
\end{tabular}

Figure S52: RP-HPLC trace of $\mathbf{1 5}$ 

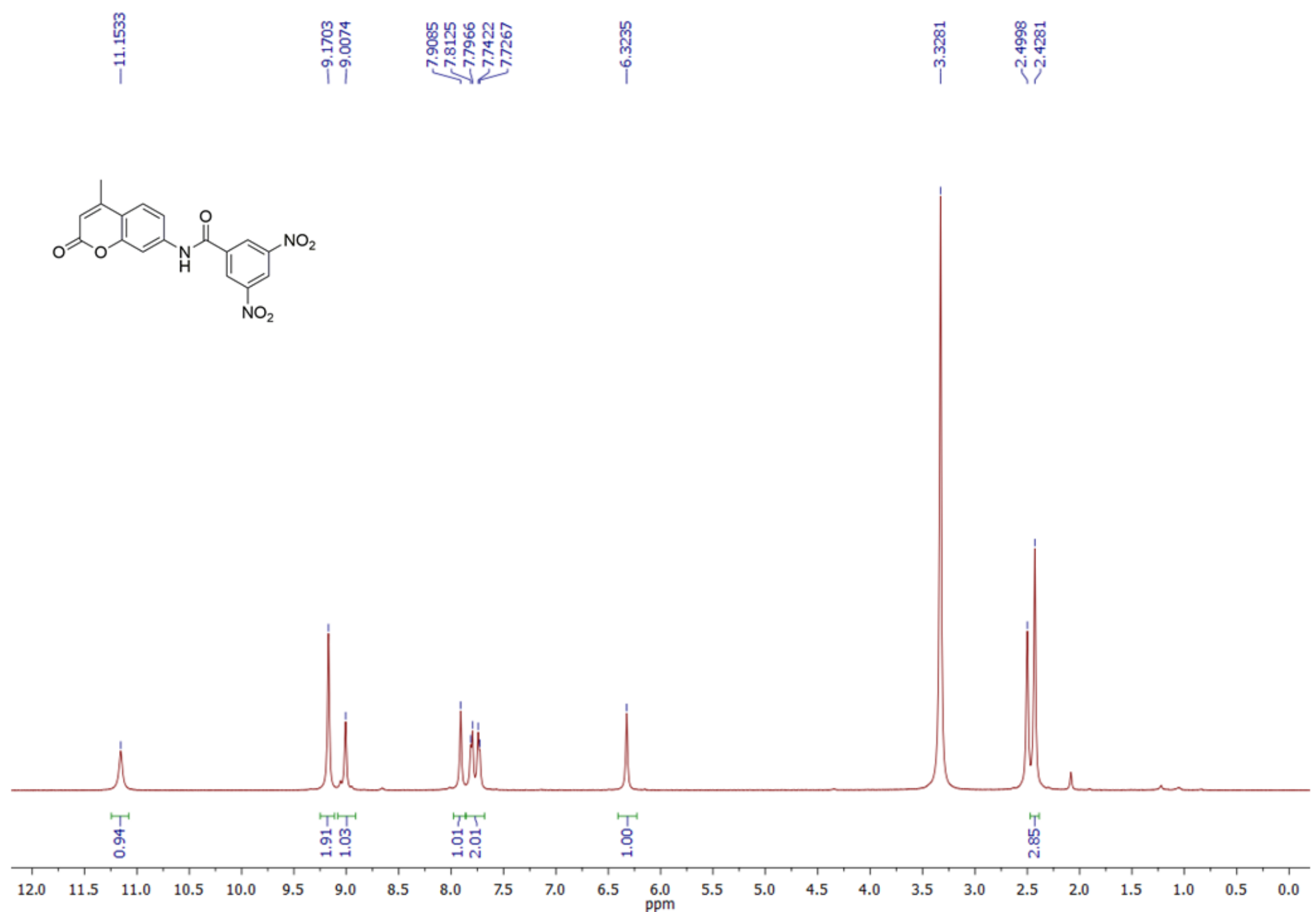

Figure S53: ${ }^{1} \mathrm{H}$ NMR $(500 \mathrm{MHz})$ spectrum of 16 in DMSO- $d_{6}$. 

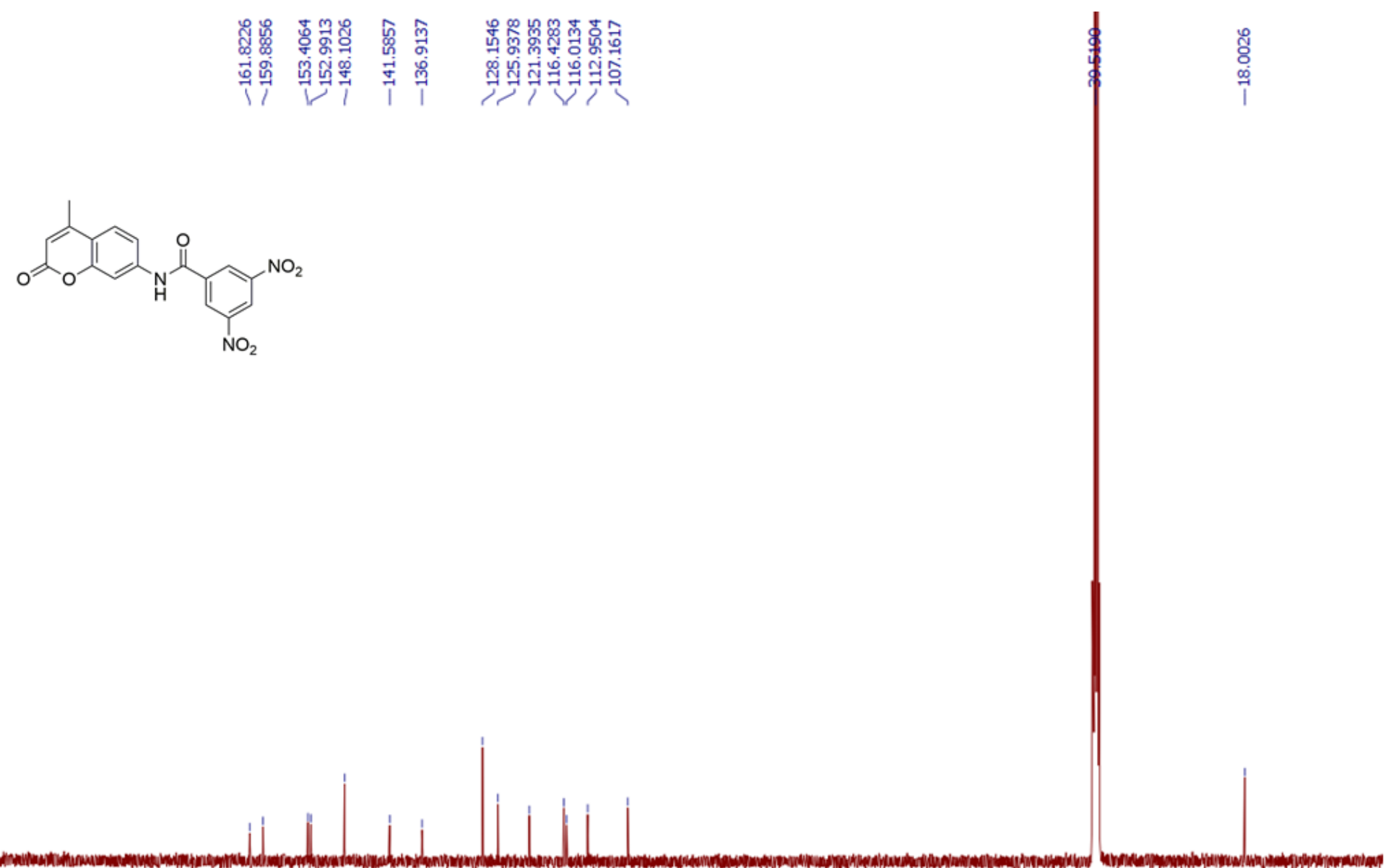

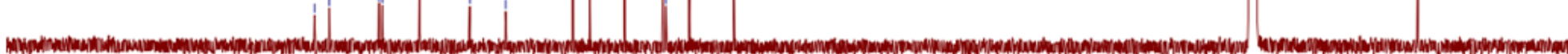

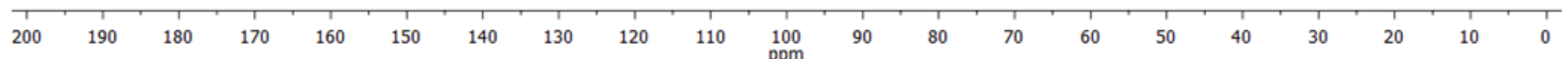

Figure S54: ${ }^{13} \mathrm{C}$ NMR $(125 \mathrm{MHz})$ spectrum of 16 in DMSO- $d_{6}$. 
+TOF MS: 0.3996 to $0.4089 \mathrm{~min}$ from Sample 3 (SON04-037D) of SONO4-037. wiff
$\mathrm{a}=7.02662322672233720 \mathrm{e}-004$, f0

Max. $5.5 e 4 \mathrm{cps}$.

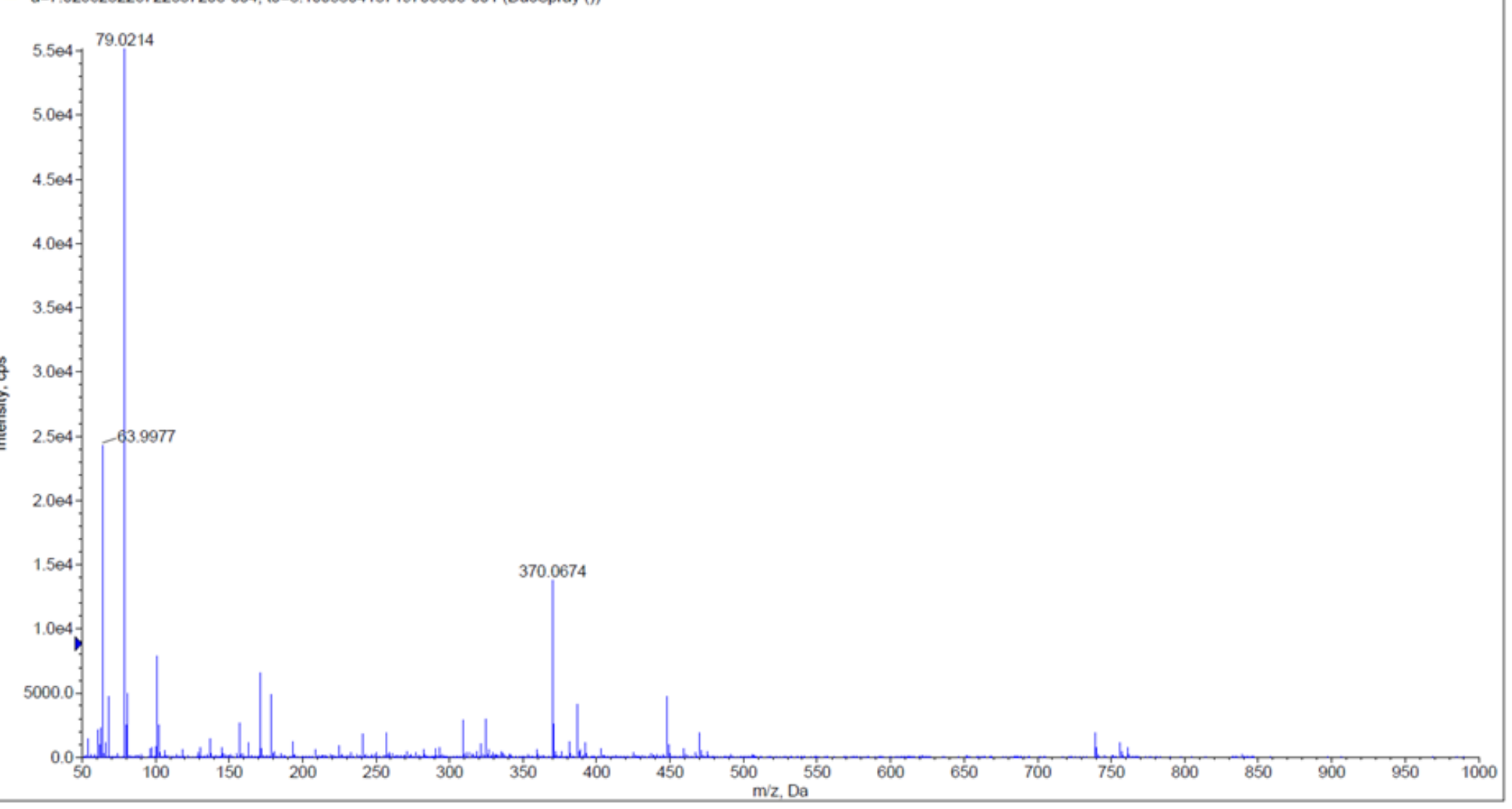

Figure S55: HRMS spectrum for 16. 


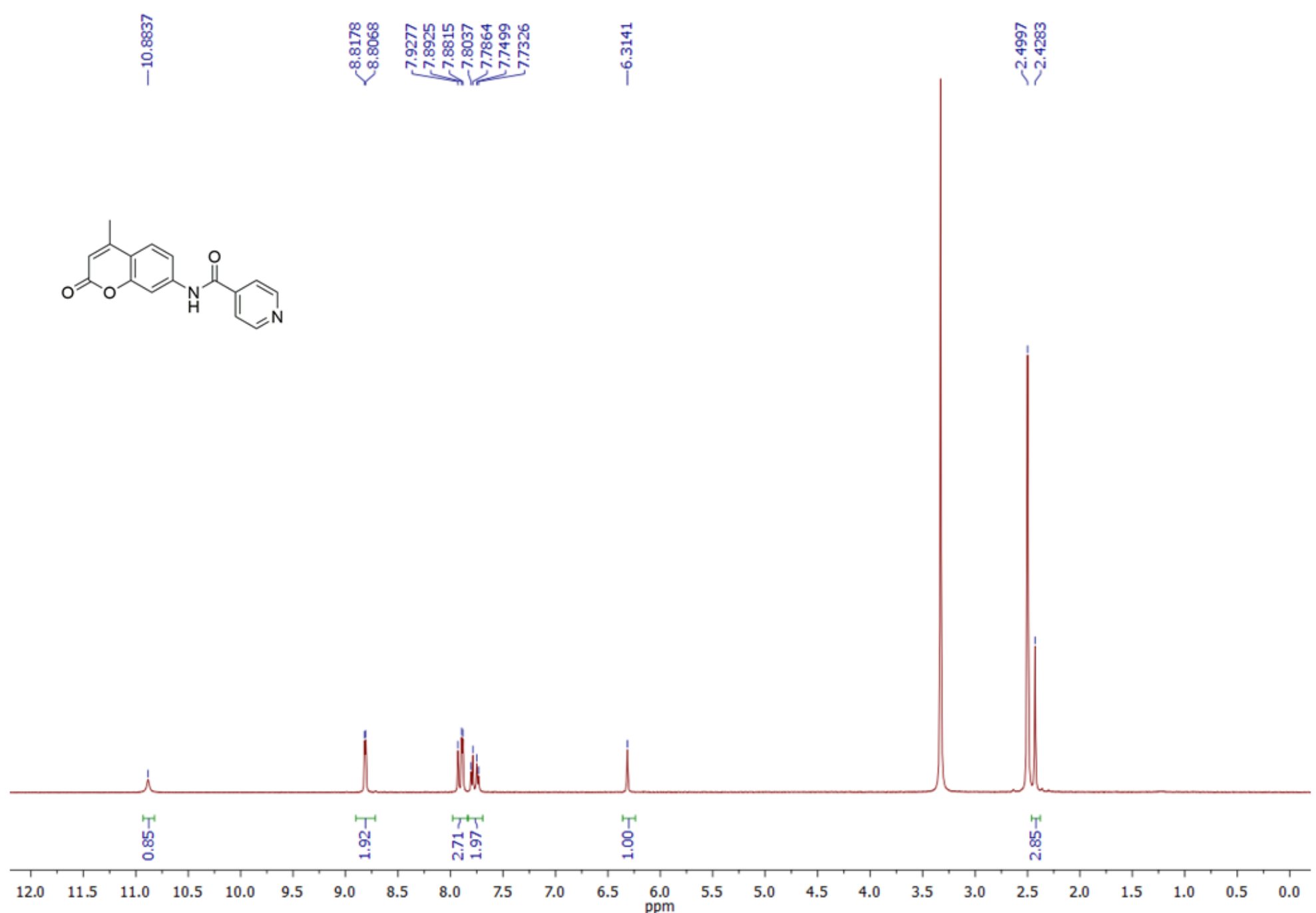

Figure S56: ${ }^{1} \mathrm{H}$ NMR (500 MHz) spectrum of 17 in DMSO- $d_{6}$ 

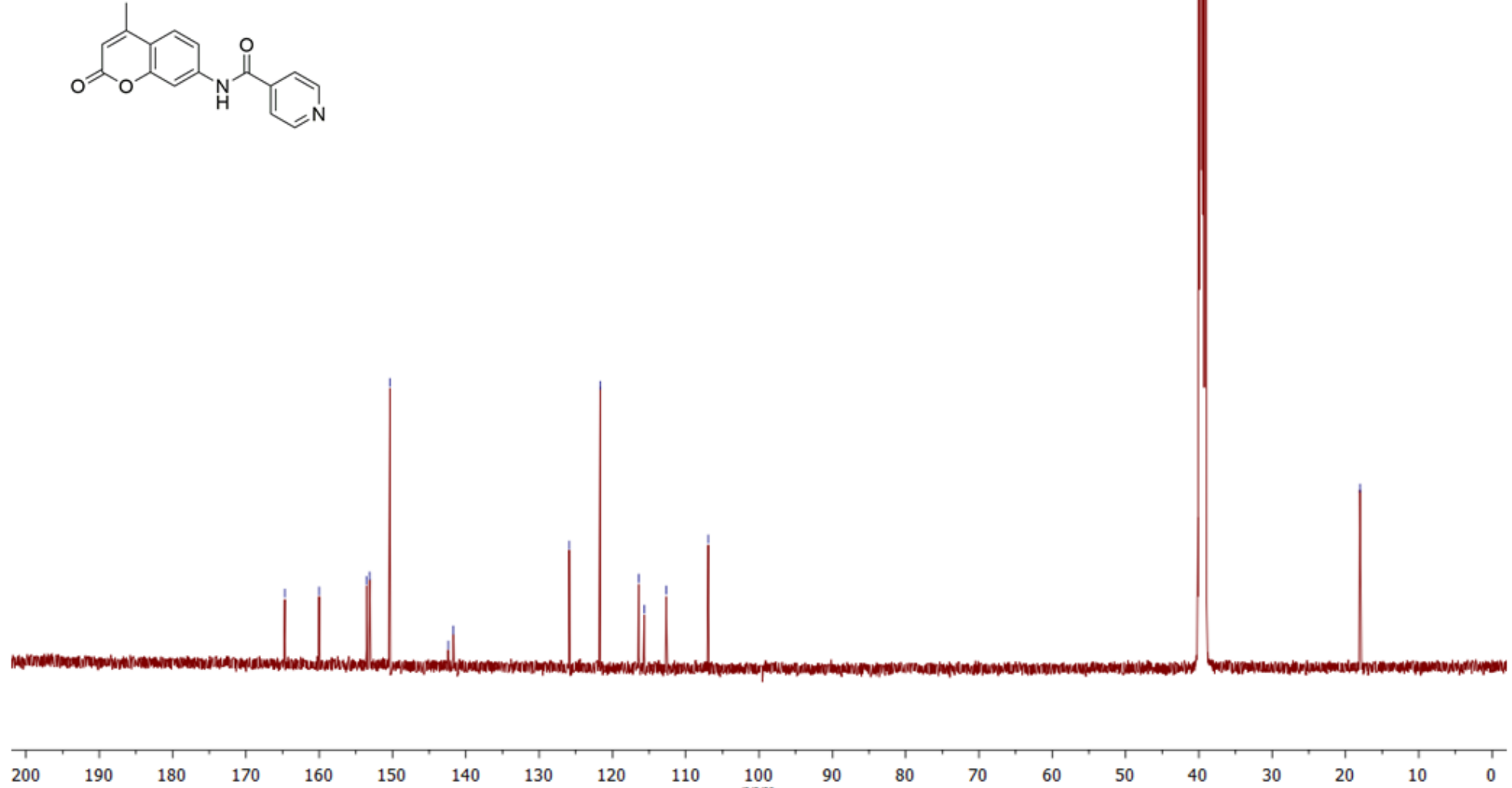

Figure S57: ${ }^{13} \mathrm{C}$ NMR $(125 \mathrm{MHz})$ spectrum of 17 in DMSO- $d_{6}$ 


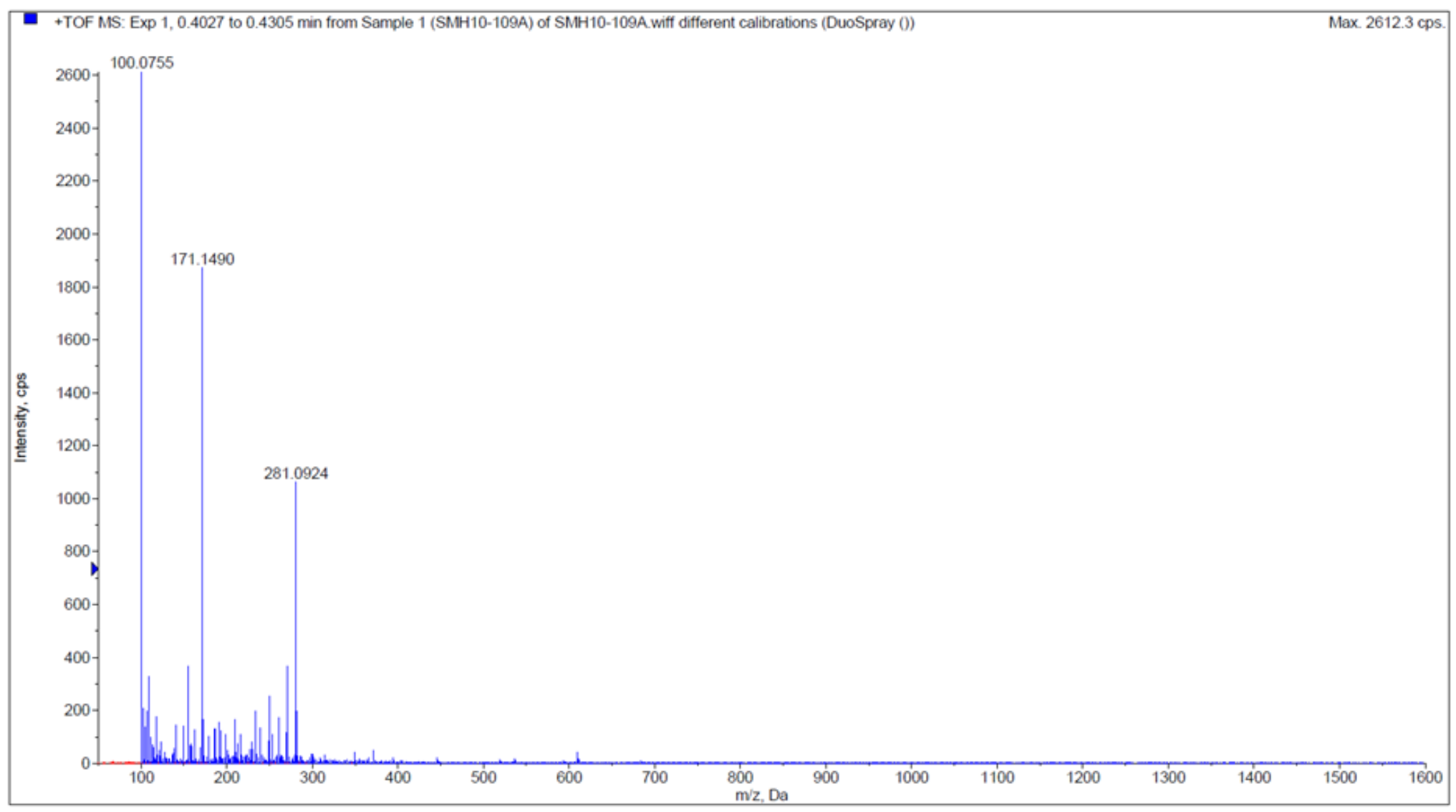

Figure S58: HRMS spectrum of 17. 
Chromatogram

SMH10-109A SMH10-109A.led

mAU

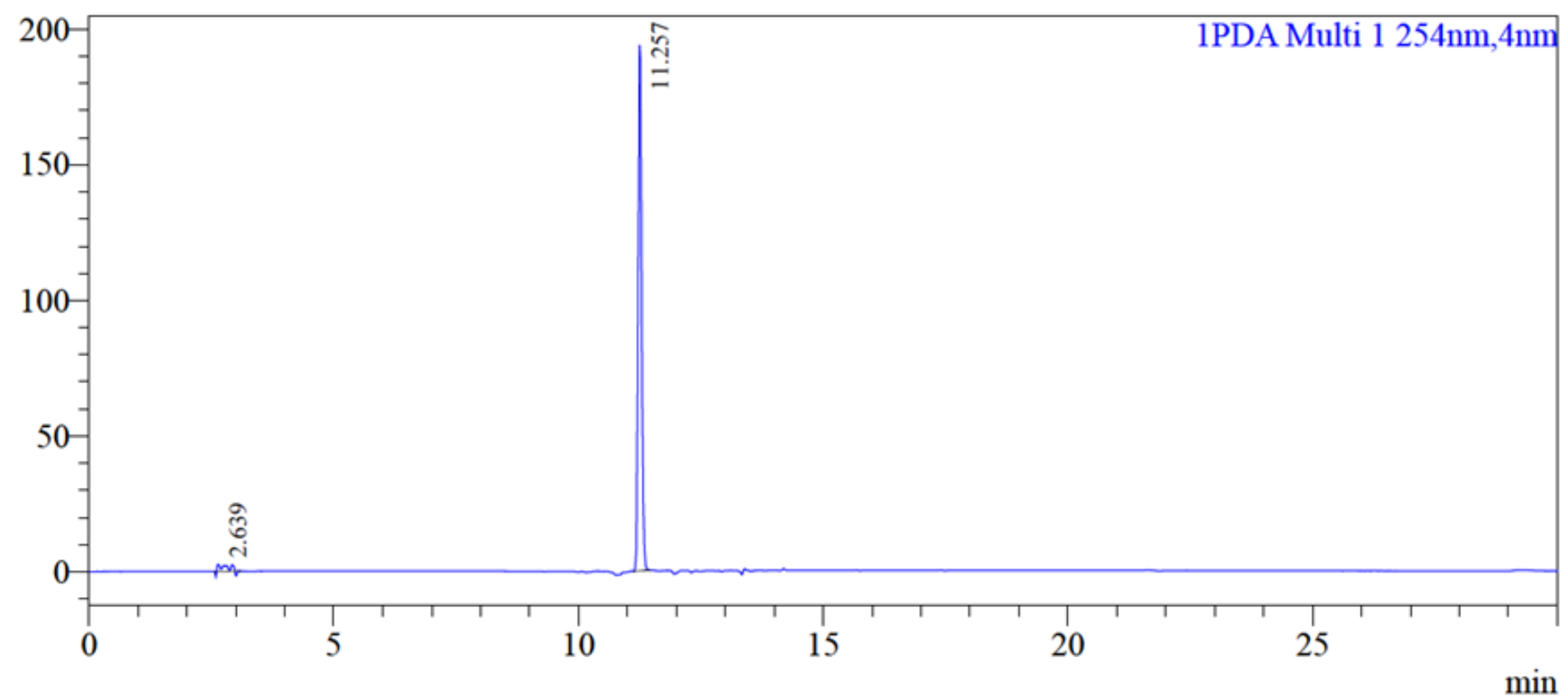

Peak Table

PDA Ch1 $254 \mathrm{~nm}$
\begin{tabular}{|r|r|r|r|r|r|}
\hline Peak\# & Ret. Time & \multicolumn{1}{|c|}{ Area } & Height & \multicolumn{1}{c|}{ Conc. } & Name \\
\hline 1 & 2.639 & 29445 & 2583 & 2.964 & \\
\hline 2 & 11.257 & 963966 & 193693 & 97.036 & \\
\hline Total & & 993410 & 196275 & & \\
\hline
\end{tabular}

Figure S59: RP-HPLC trace of 17. 


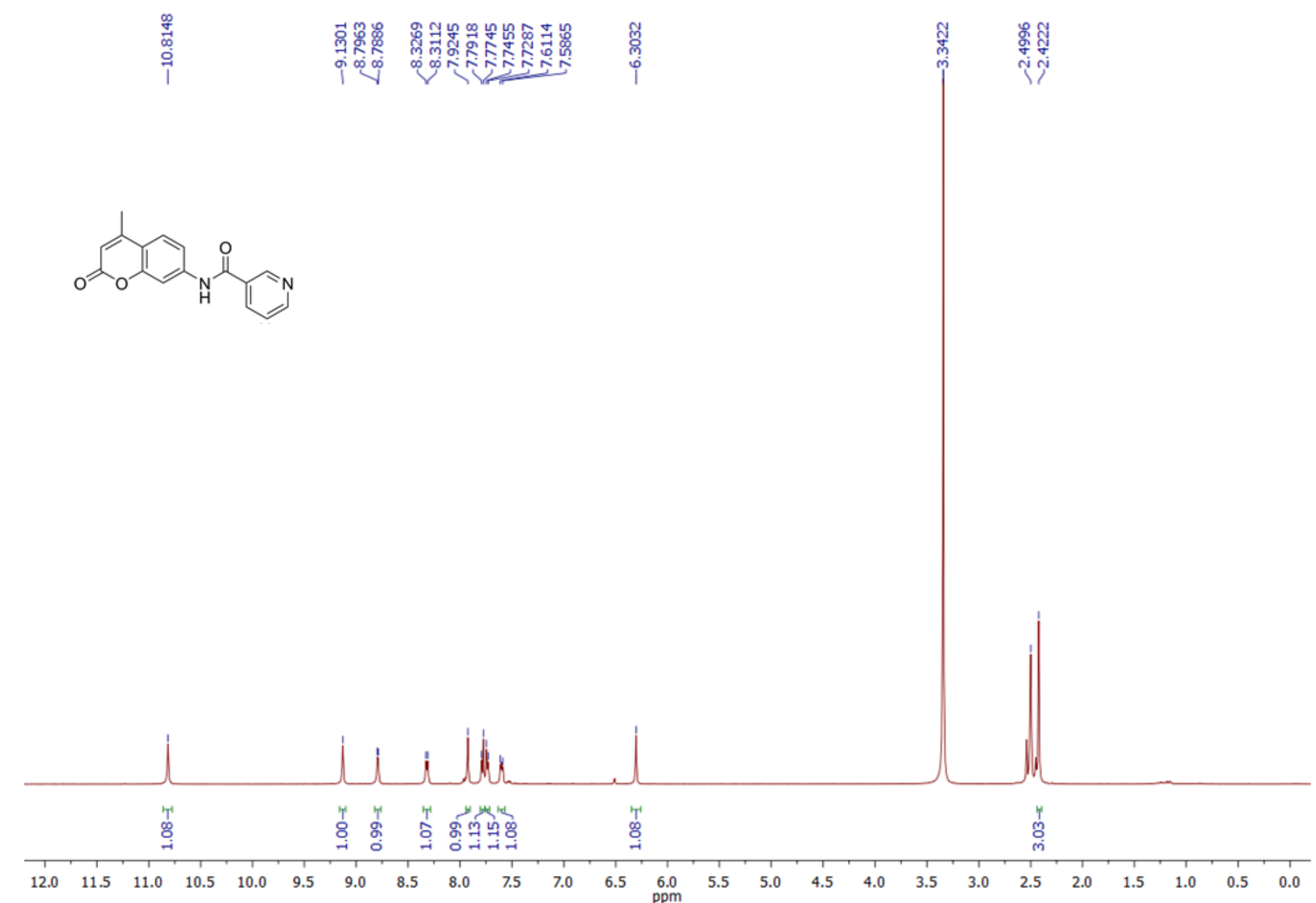

Figure S60: ${ }^{1} \mathrm{H}$ NMR $(500 \mathrm{MHz})$ spectrum of 18 in DMSO- $d_{6}$. 


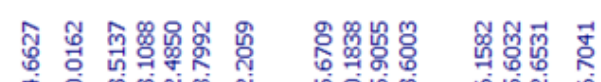

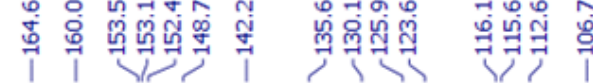
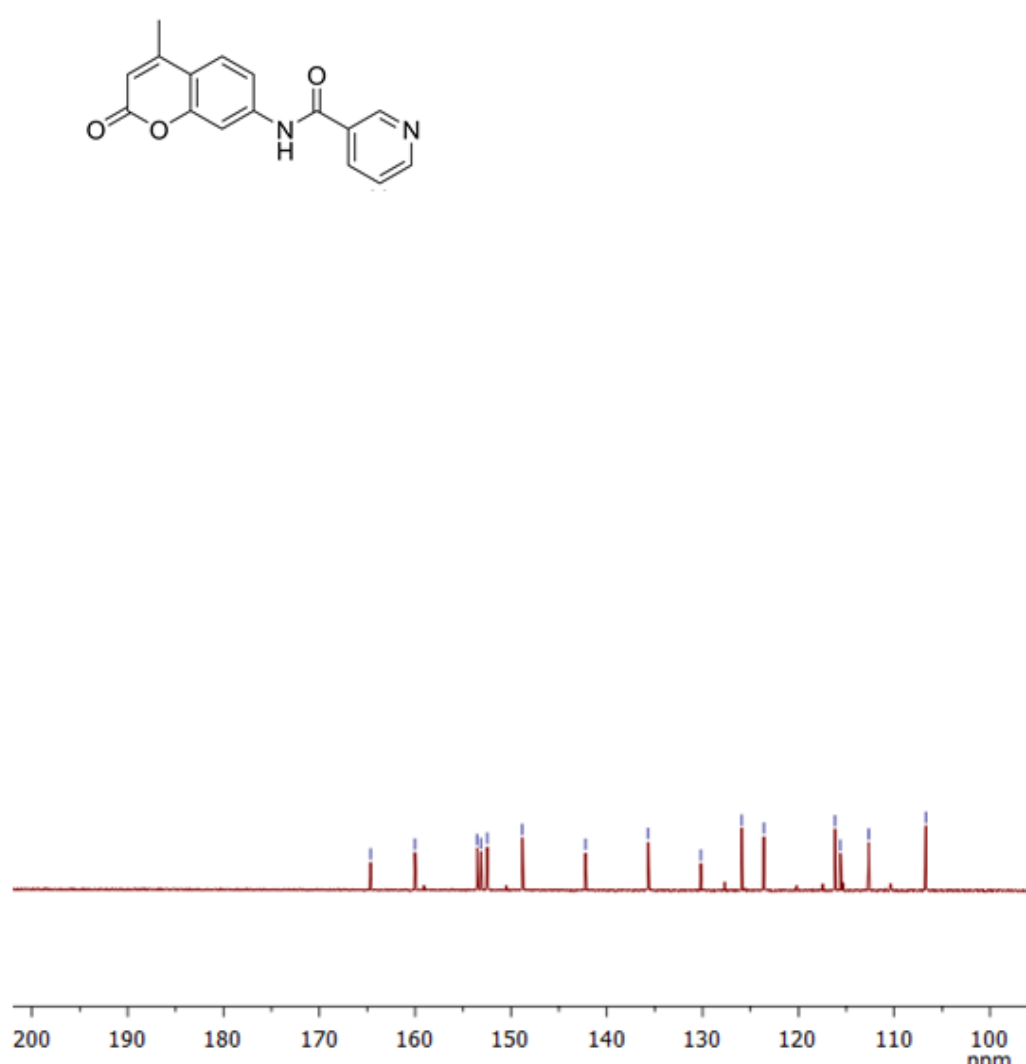

Figure S61: ${ }^{13} \mathrm{C}$ NMR $(125 \mathrm{MHz})$ spectrum of 18 in DMSO- $d_{6}$ 
- +TOF MS: Exp 1, 0.3934 to 0.4212 min from Sample 1 (SMH11-013A) of SMH11-013A.wiff different calibrations (DuoSpray ())

Max. $7287.5 \mathrm{cps}$.

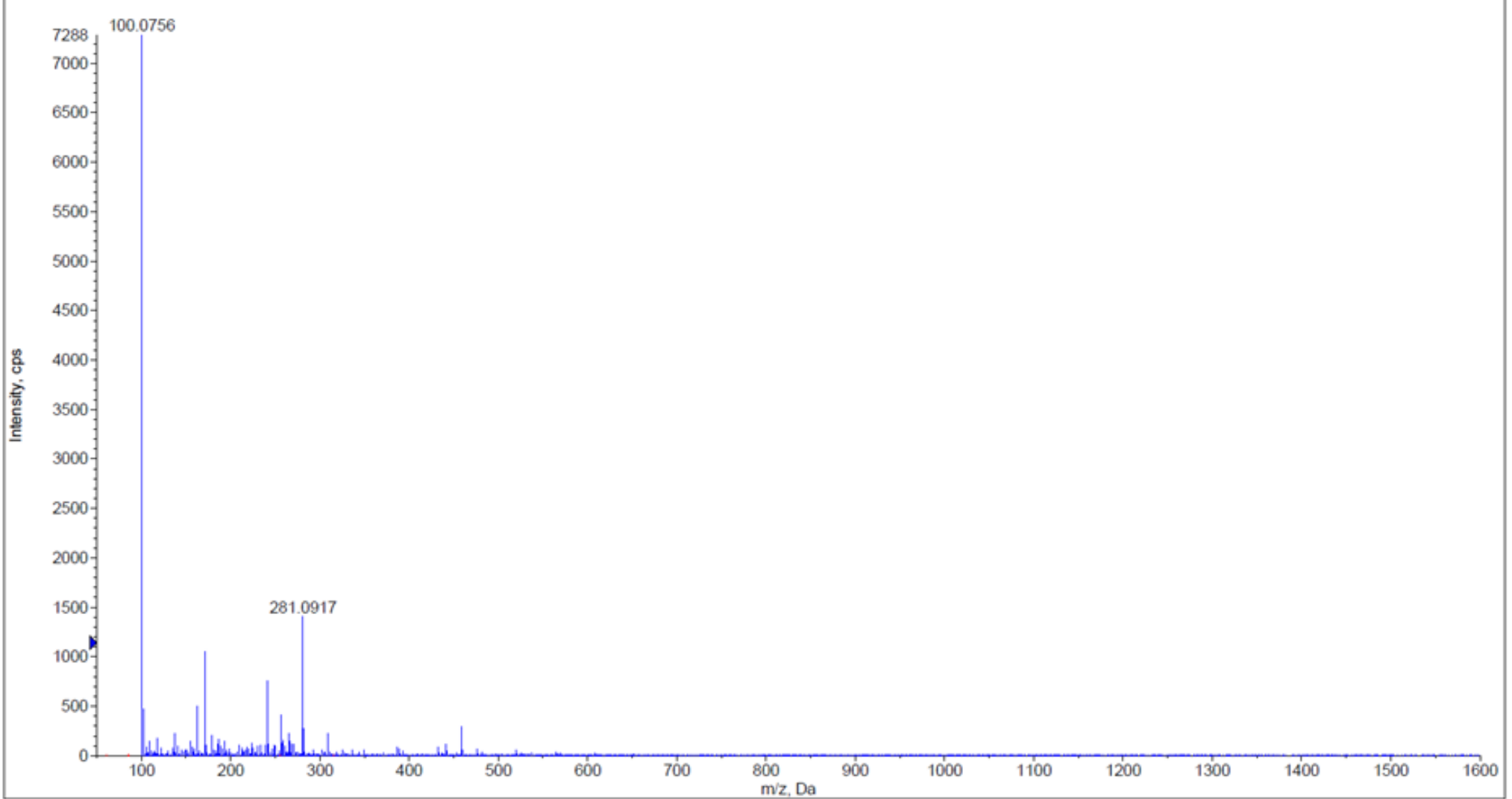

Figure S62: HRMS spectrum of 18. 
Chromatogram

mAU

SMH11-003B SMH11-003B.led

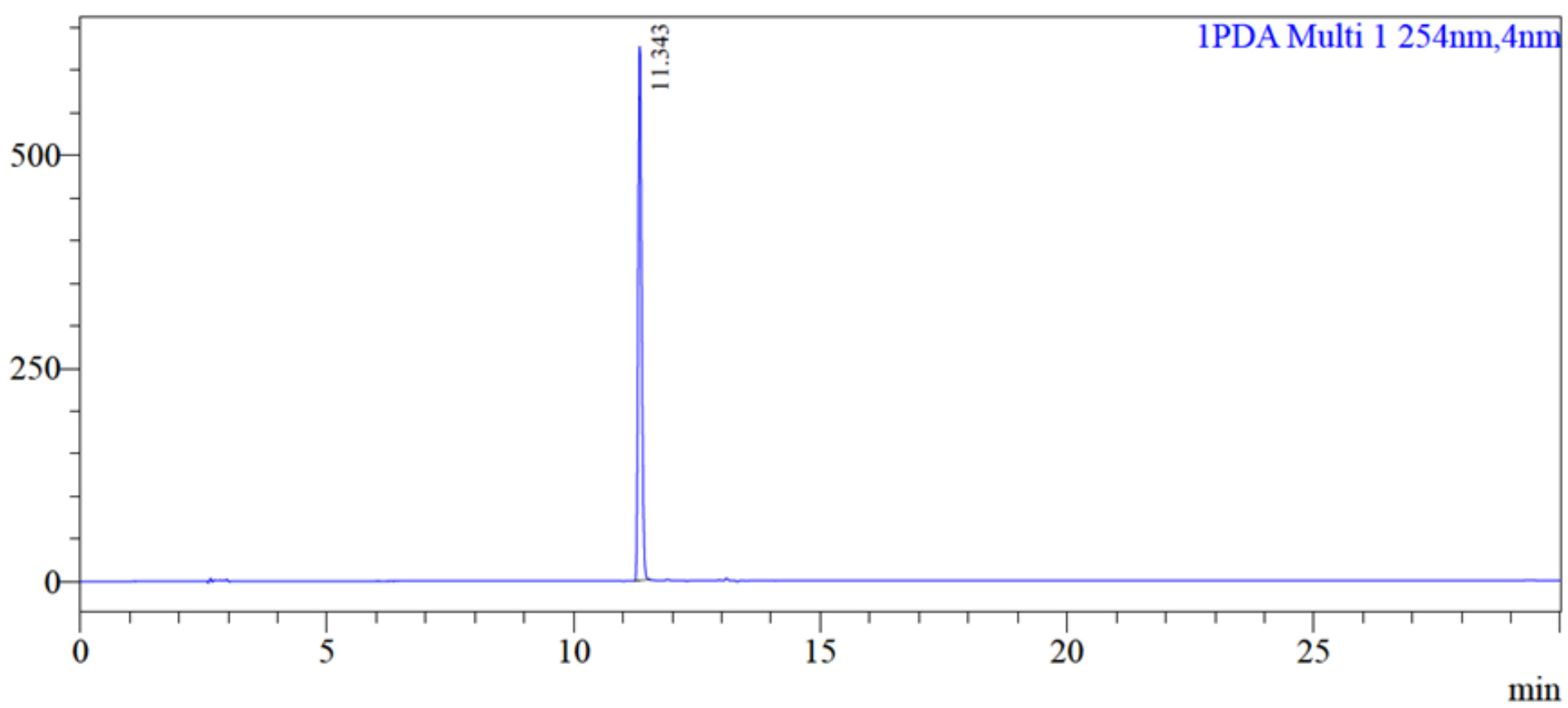

Peak Table

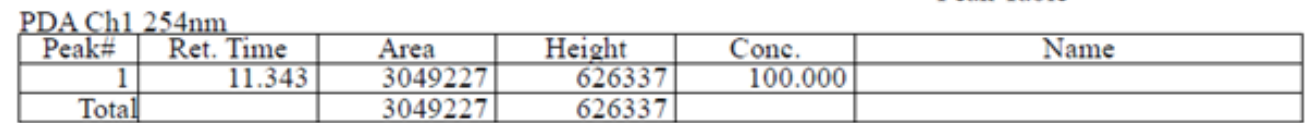

Figure S63: RP-HPLC trace of 18. 


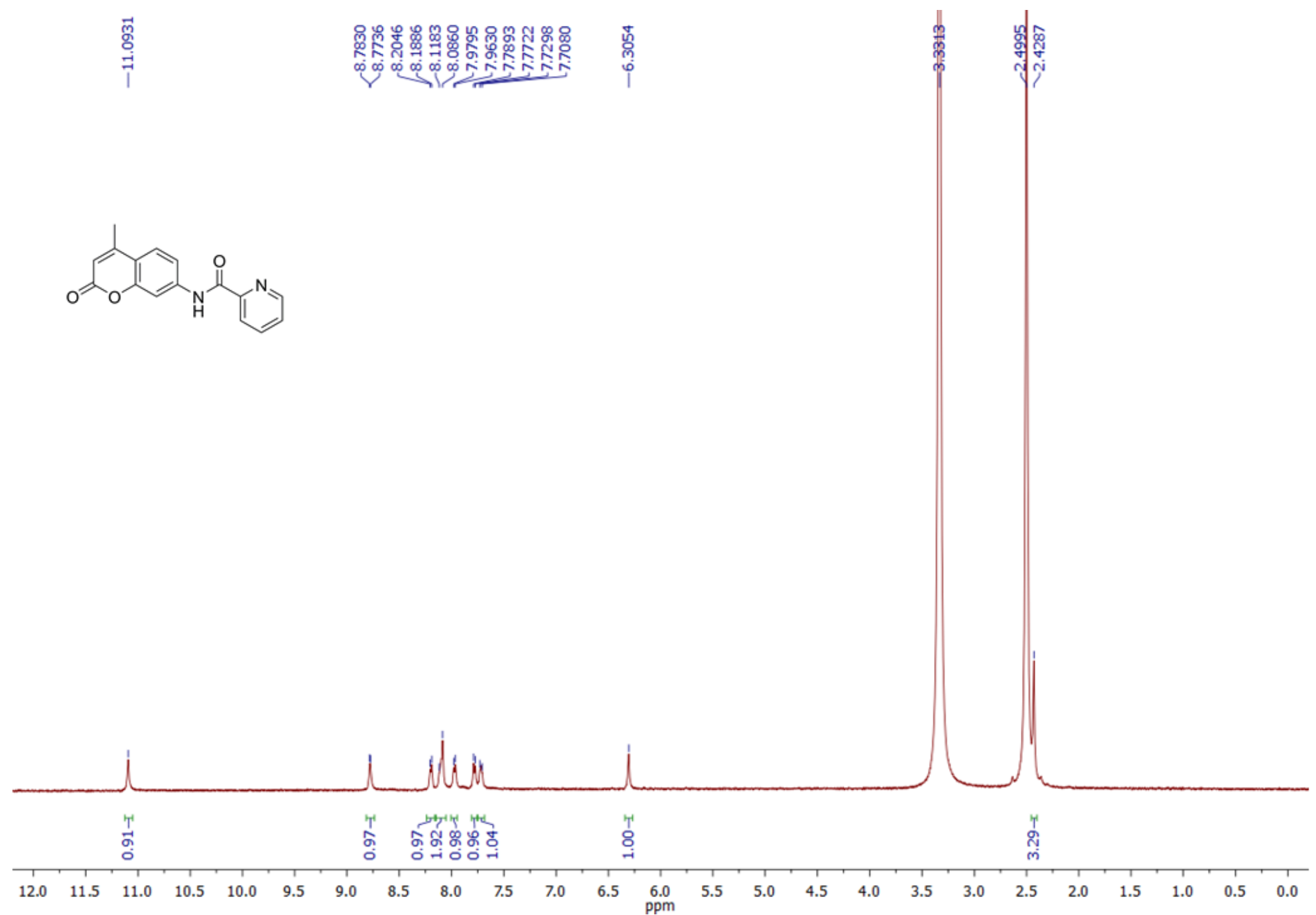

Figure S64: ${ }^{1} \mathrm{H}$ NMR $(500 \mathrm{MHz})$ spectrum of 19 in DMSO- $d_{6}$. 

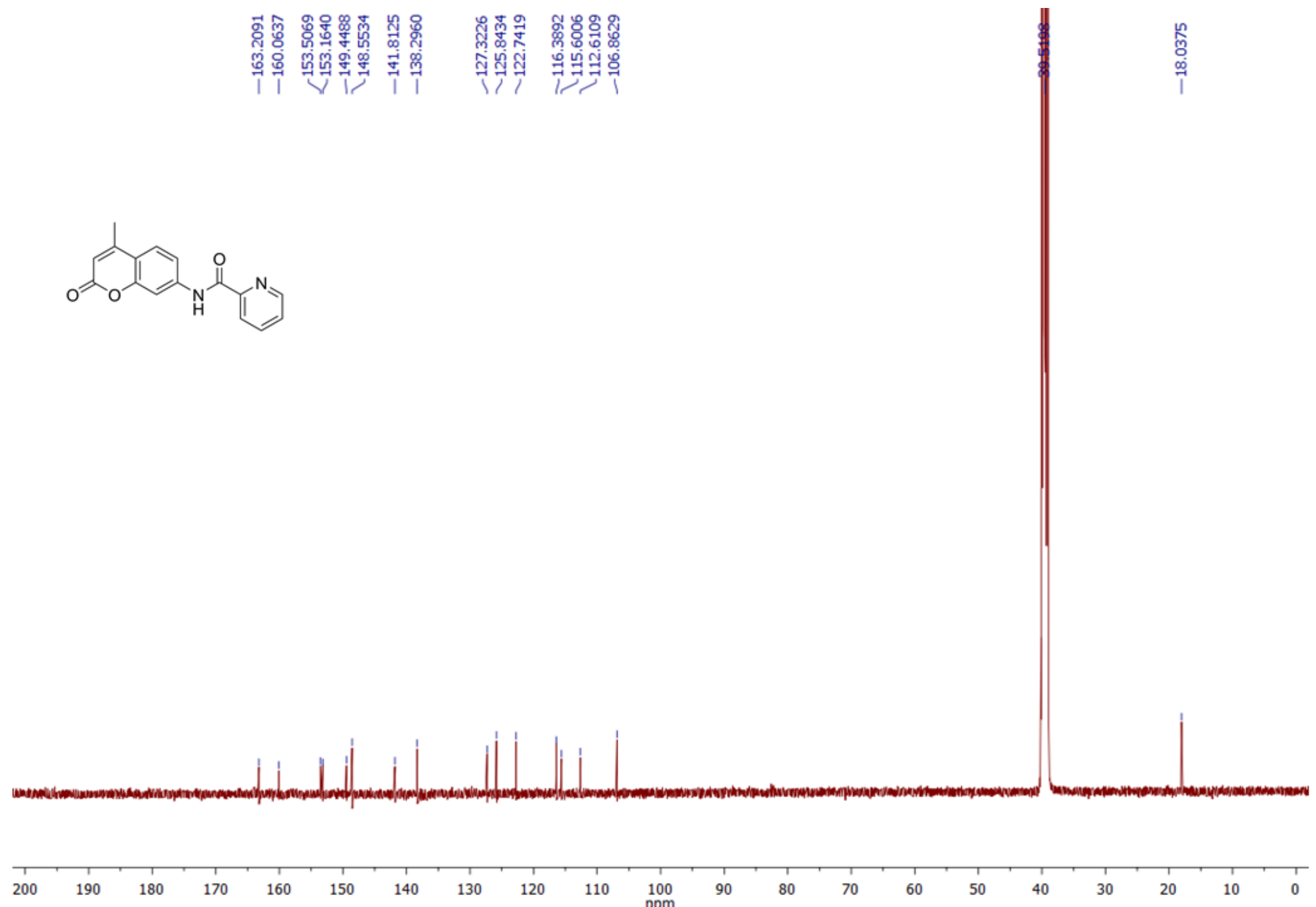

Figure S65: ${ }^{13} \mathrm{C}$ NMR (125 MHz) spectrum of 19 in DMSO- $d_{6}$. 
- +TOF MS: Exp 1, 0.4119 to 0.4490 min from Sample 1 (SMH11-011A) of SMH11-011A.wiff different calibrations (DuoSpray ())

Max. $7193.6 \mathrm{cps}$.

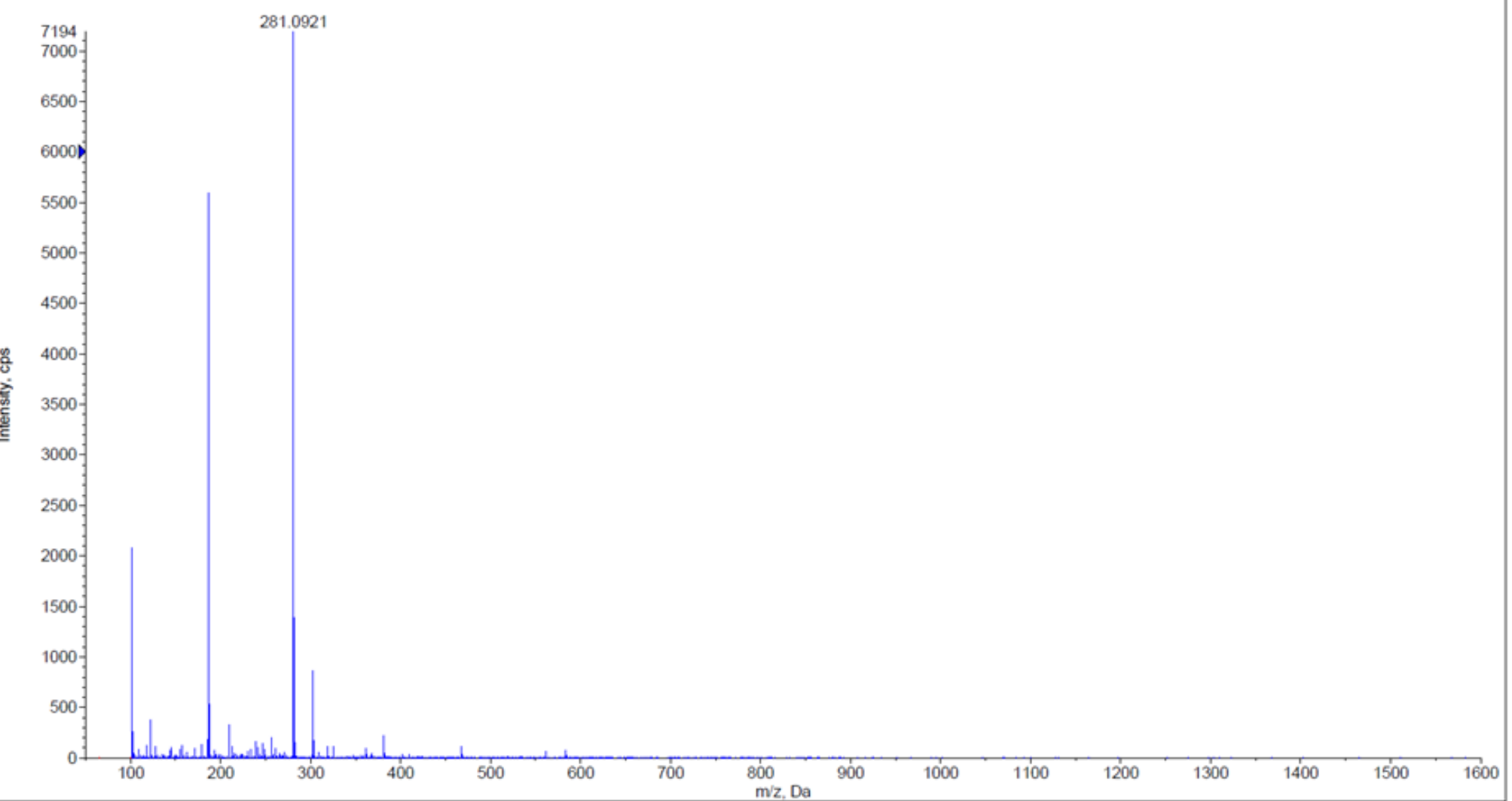

Figure S66: HRMS spectrum of 19. 
Chromatogram

SMH11-011A SMH11-011A.lcd

mAU

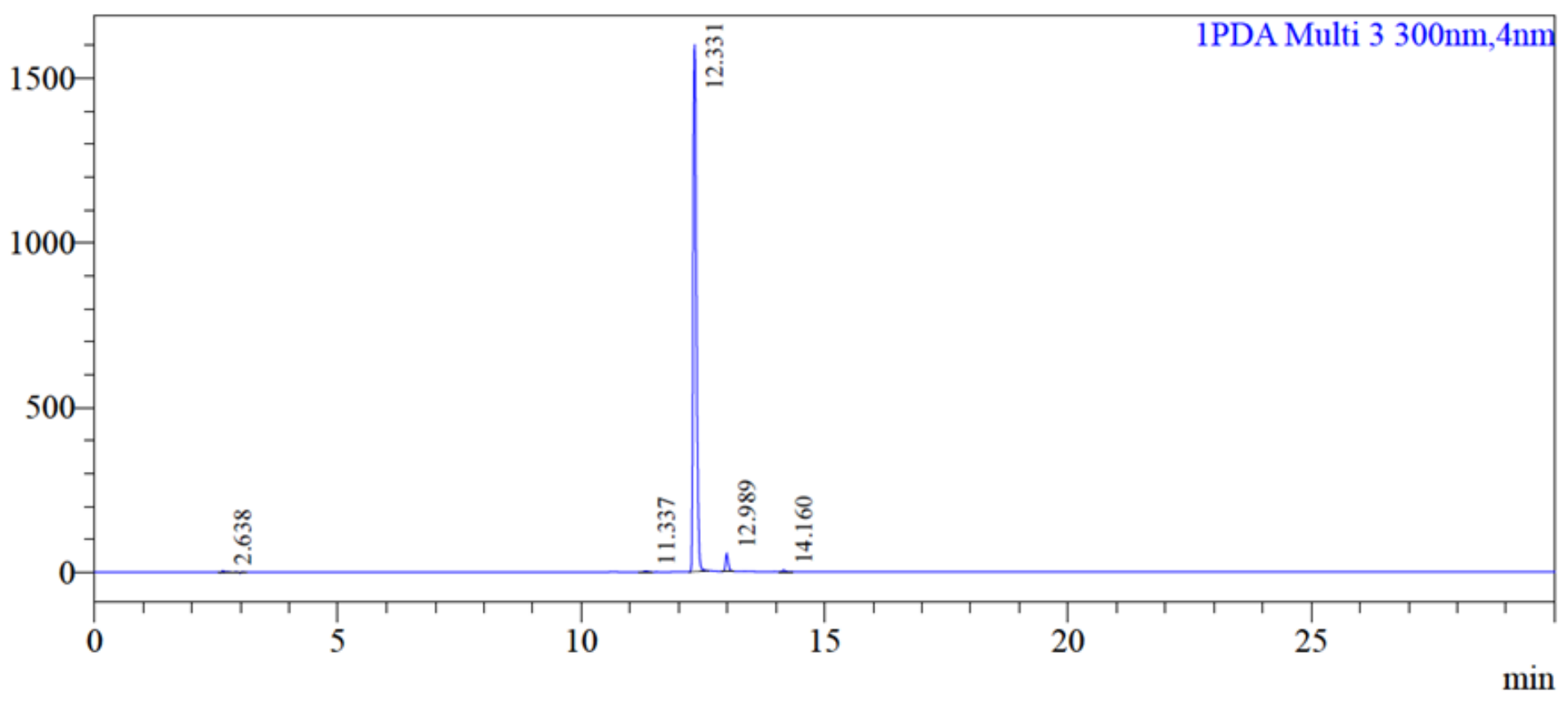

Peak Table

PDA. Ch3 300 nm
\begin{tabular}{|r|r|r|r|r|r|}
\hline Peak $\#$ & Ret. Time & \multicolumn{1}{|c|}{ Area } & Height & Conc. & \\
\hline 1 & 2.638 & 6363 & 4621 & 0.081 & \\
\hline 2 & 11.337 & 13306 & 2871 & 0.170 & \\
\hline 3 & 12.331 & 7586936 & 1601292 & 96.907 & \\
\hline 4 & 12.989 & 204805 & 53368 & 2.616 & \\
\hline 5 & 14.160 & 17706 & 5103 & 0.226 & \\
\hline Total & & 7829117 & 1667255 & & \\
\hline
\end{tabular}

Figure S67: RP-HPLC trace for 19. 


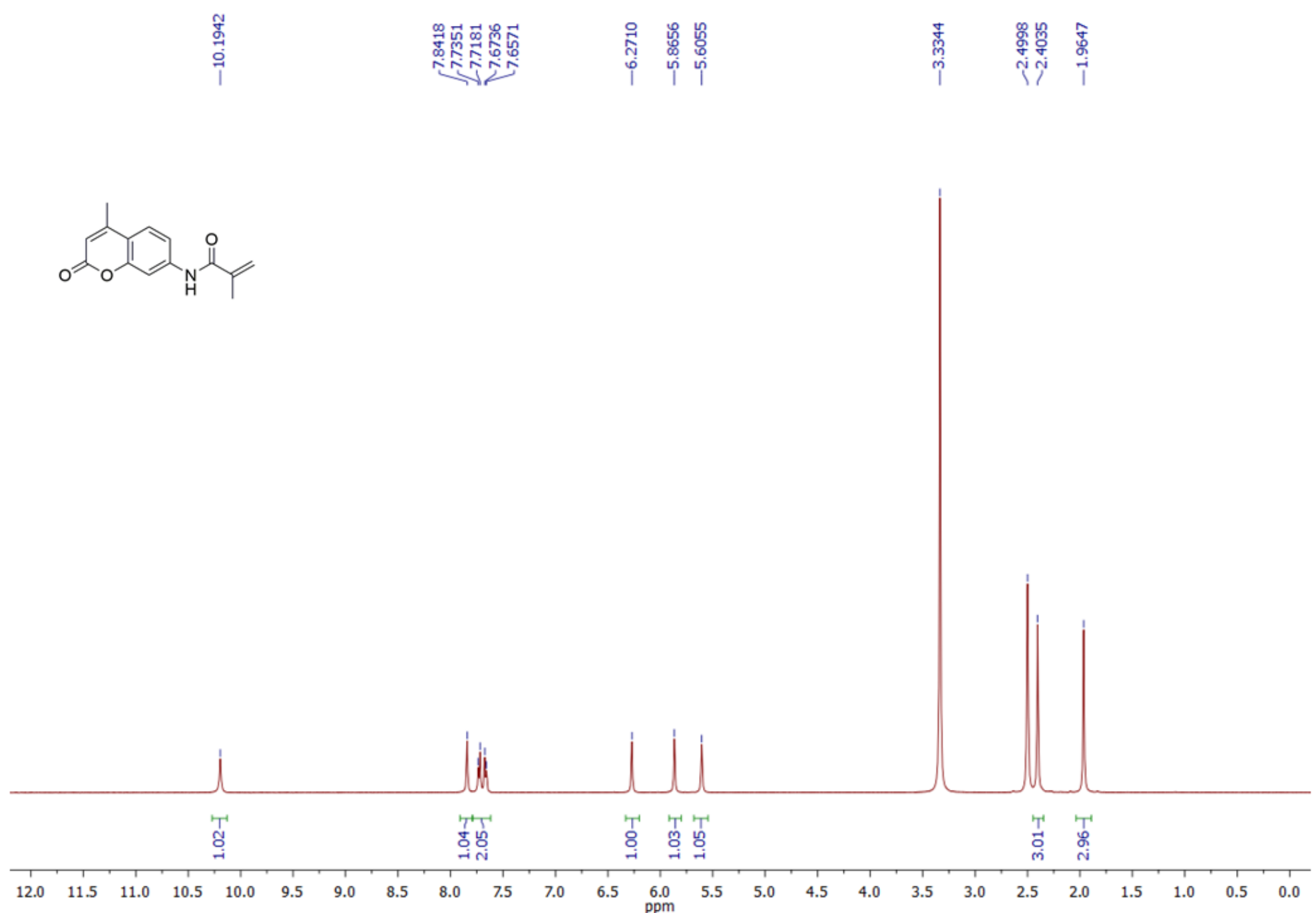

Figure S68: ${ }^{1} \mathrm{H}$ NMR $(500 \mathrm{MHz})$ spectrum of 20 in DMSO- $d_{6}$. 


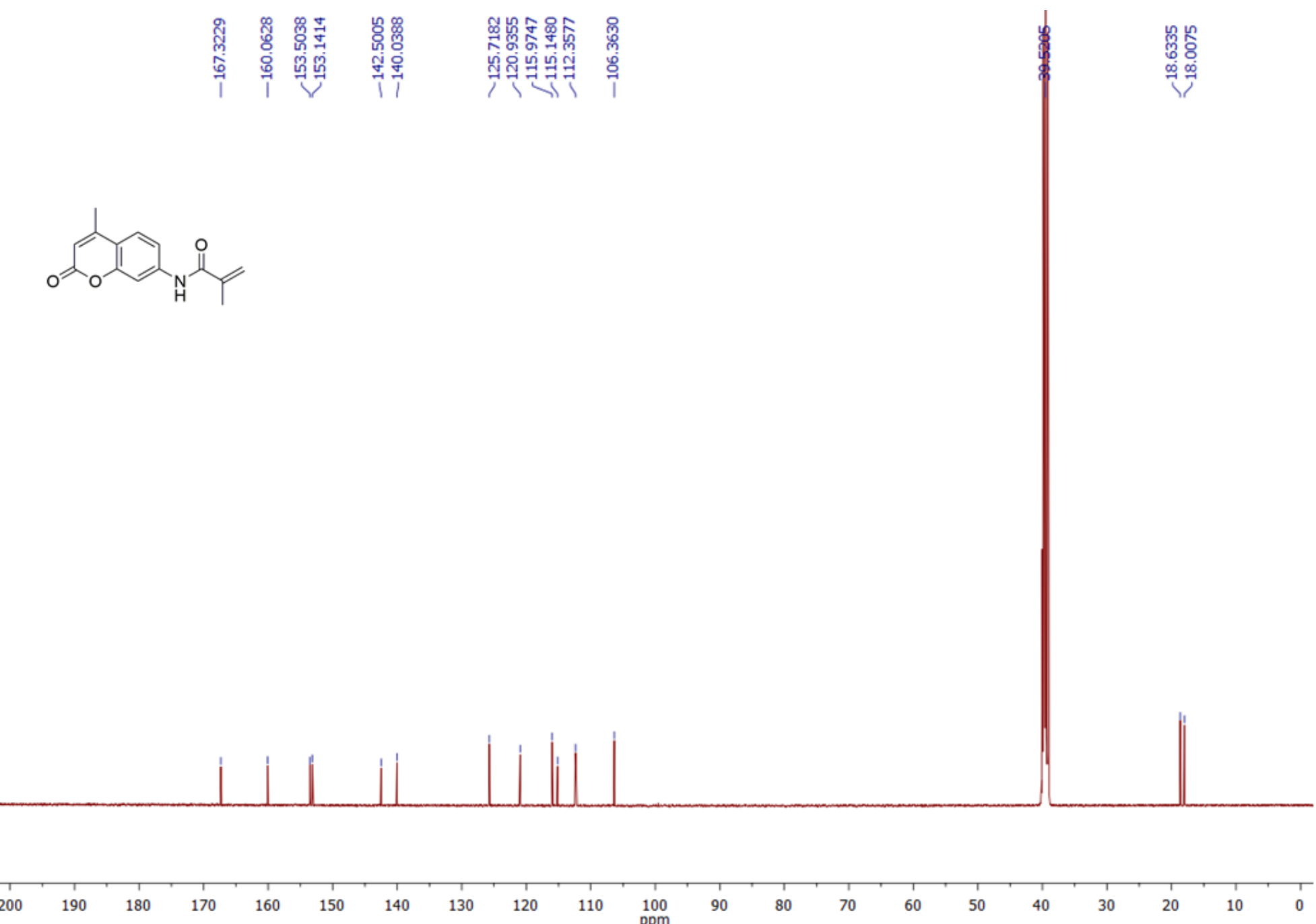

Figure S69: ${ }^{13} \mathrm{C}$ NMR $(125 \mathrm{MHz})$ spectrum of 20 in DMSO- $d_{6}$ 
-TOF MS: Exp 1, 0.3841 to 0.4212 min from Sample 1 (SMH10-087A) of SMH10-087A.wiff different calibrations (DuoSpray ())

Max. 8854.4 cps.

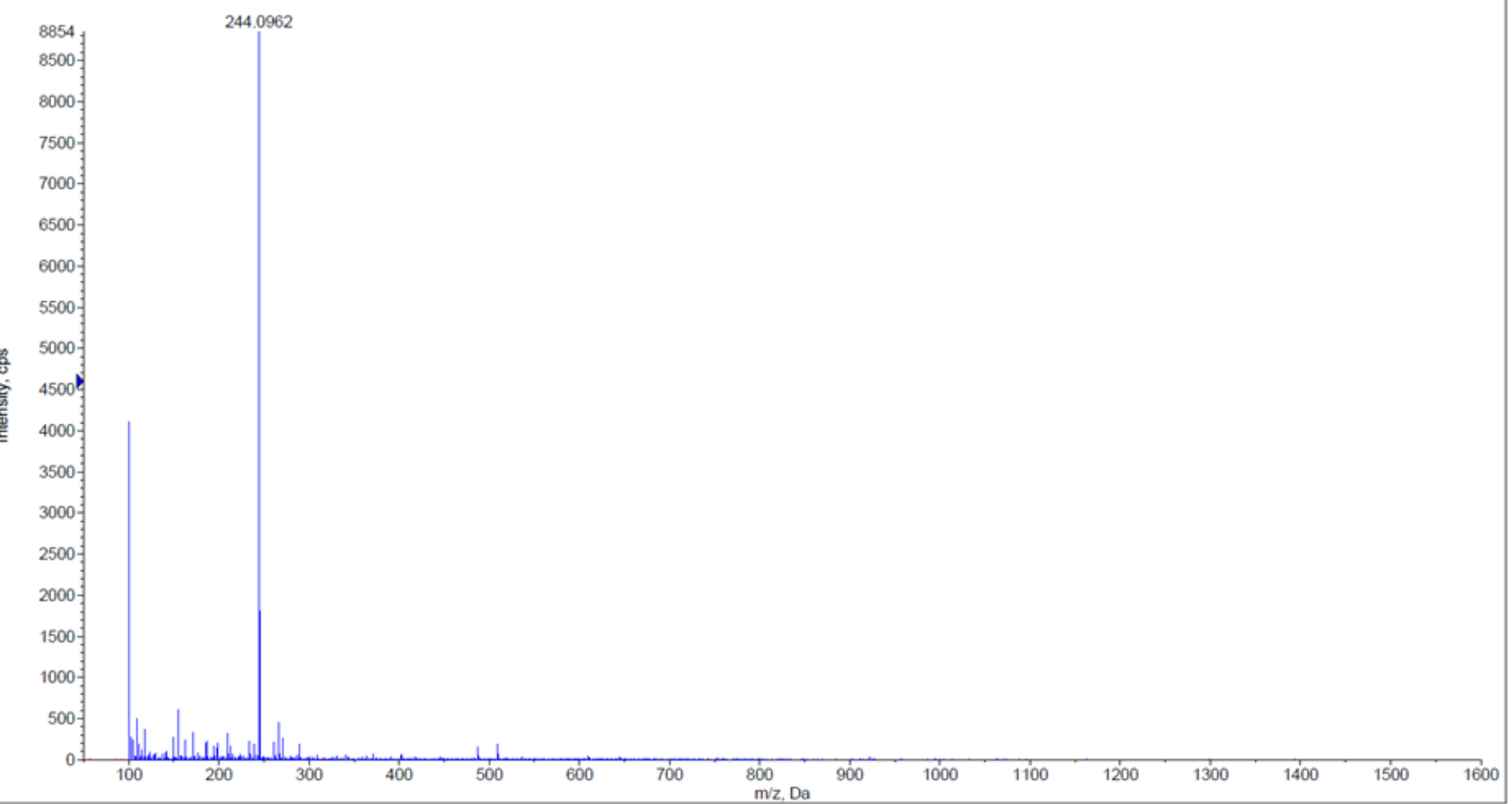

Figure S70: HRMS spectrum of 20. 
Chromatogram

SMH10-087A SMH10-087A.lcd

$\mathrm{mAU}$

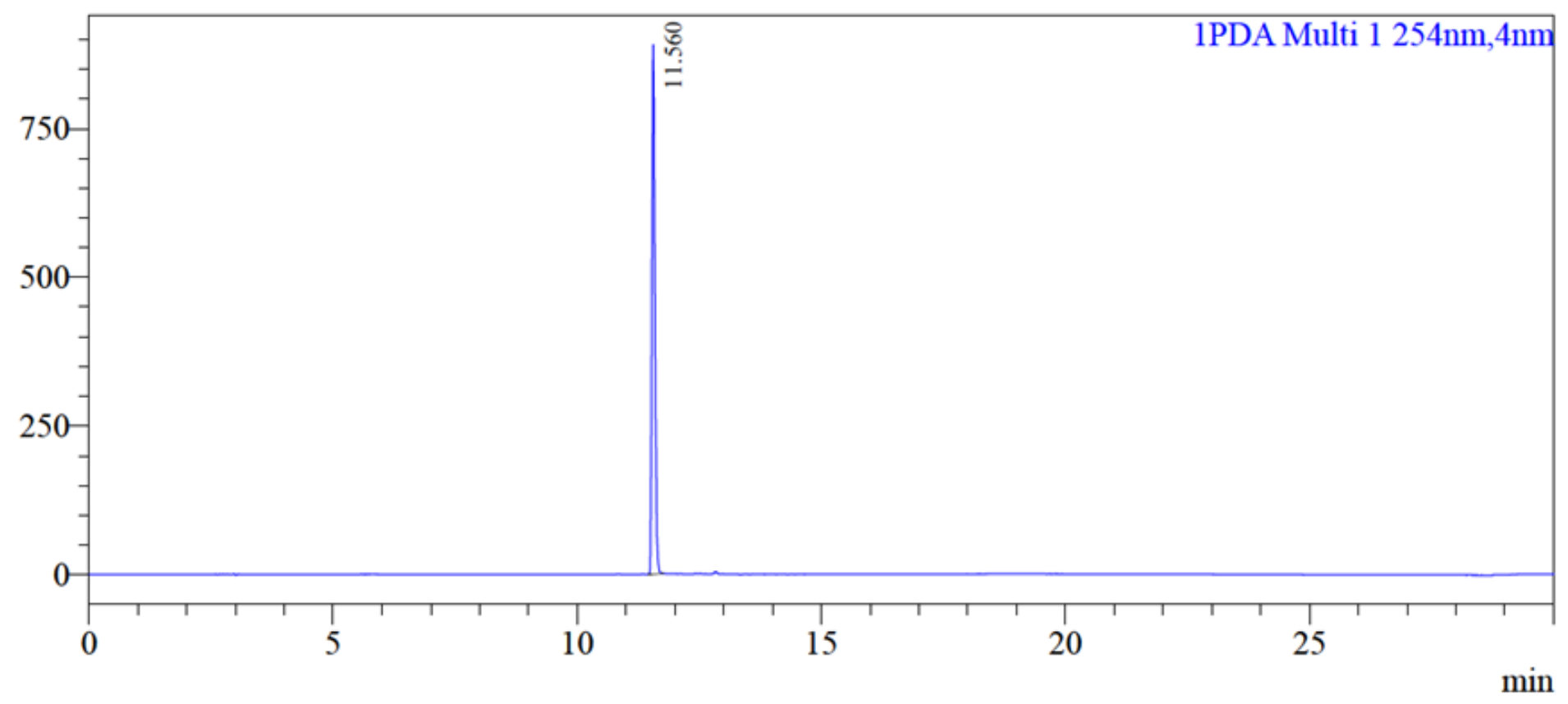

Peak Table

PDA Ch1 $254 \mathrm{~nm}$
\begin{tabular}{|r|r|r|r|r|r|}
\hline Peak= & Ret. Time & \multicolumn{1}{|c|}{ Area } & Height & Conc. & Name \\
\hline 1 & 11.560 & 3790739 & 890641 & 100.000 & \\
\hline Total & & 3790739 & 890641 & & \\
\hline
\end{tabular}

Figure S71: RP-HPLC trace of 20. 


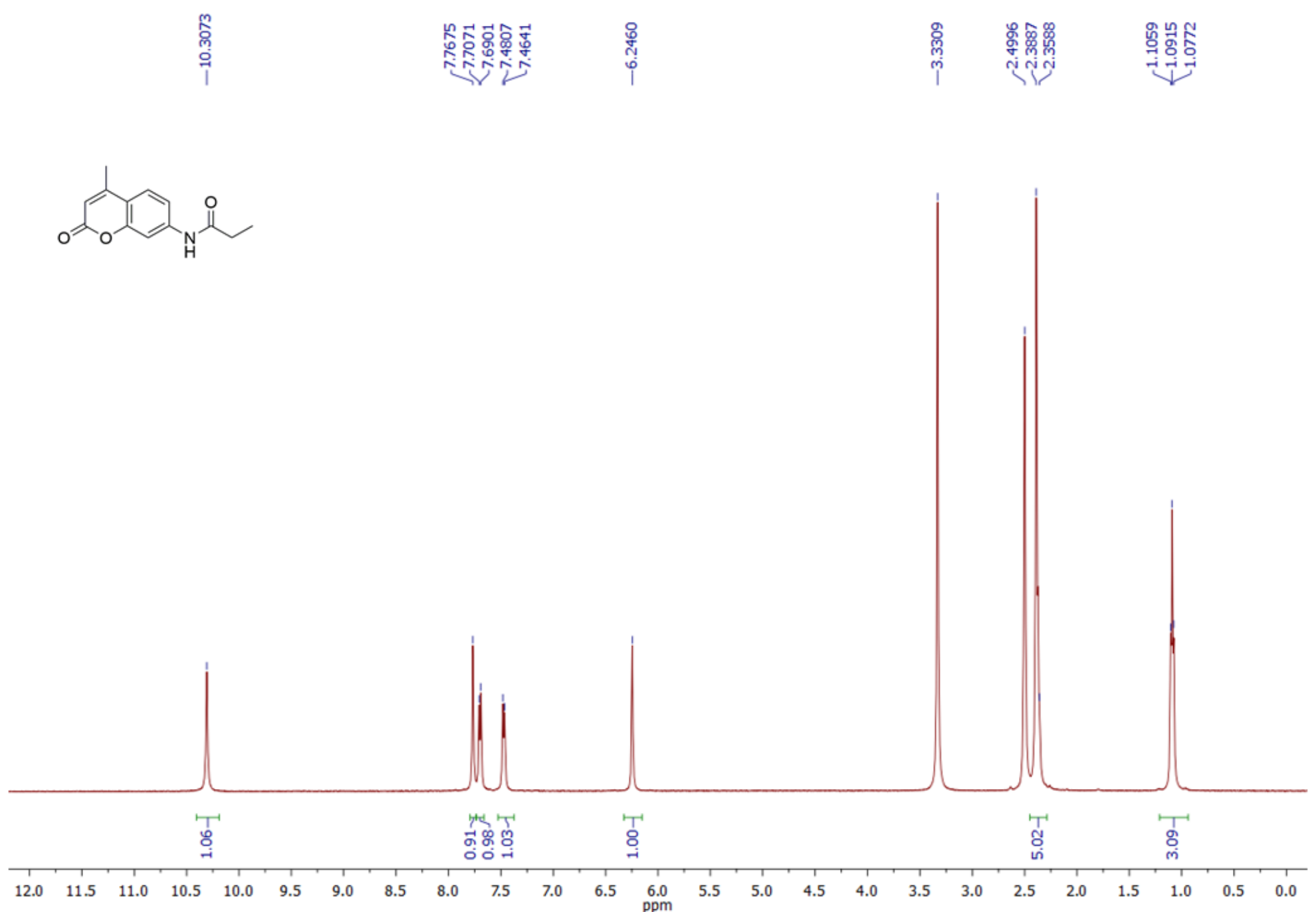

Figure S72: ${ }^{1} \mathrm{H}$ NMR $(500 \mathrm{MHz})$ spectrum of 21 in DMSO- $d_{6}$. 


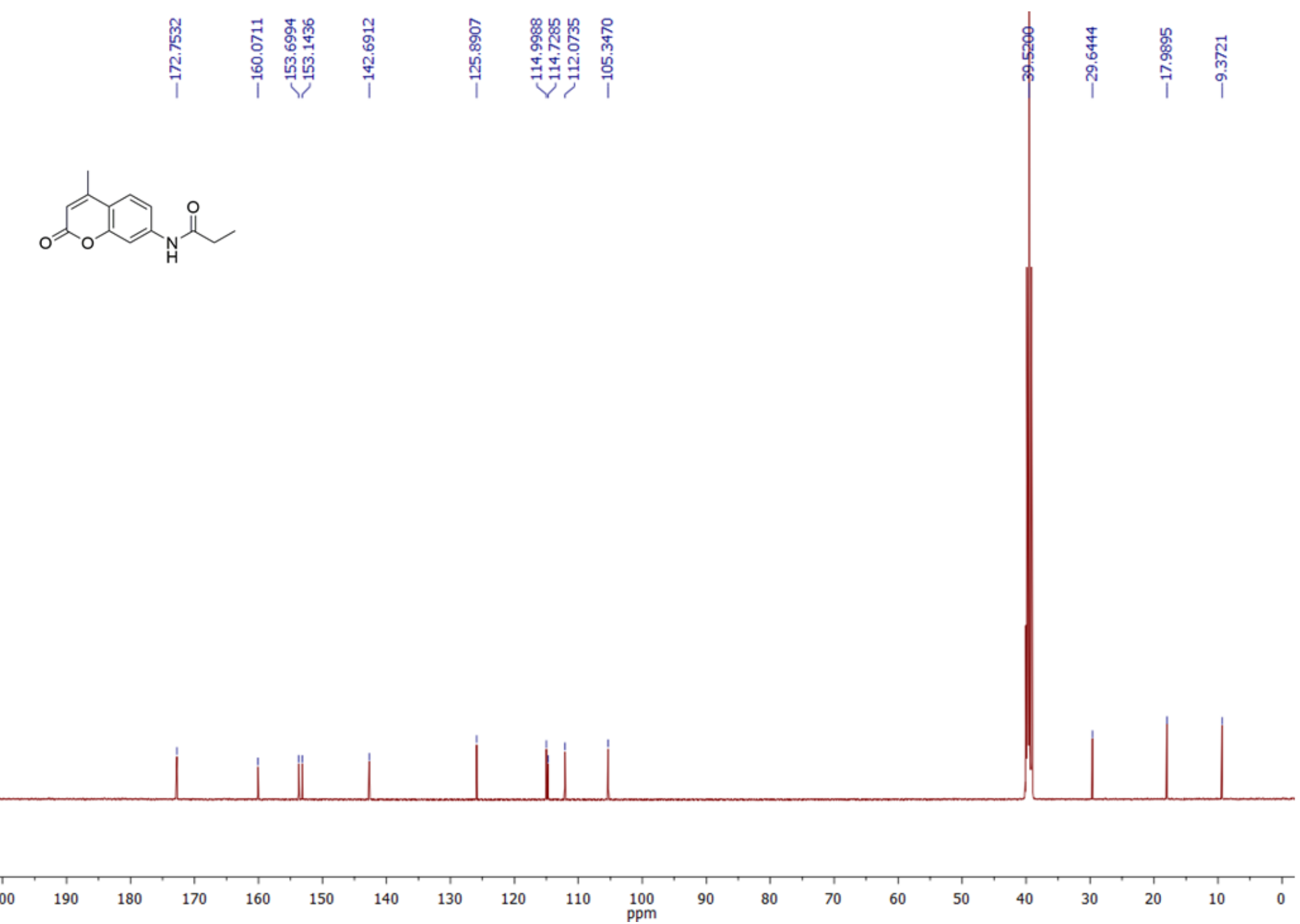

Figure S73: ${ }^{13} \mathrm{C}$ NMR $(125 \mathrm{MHz})$ spectrum of 21 in DMSO- $d_{6}$. 
+TOF MS: Exp 1, 0.2359 to 0.2637 min from Sample 1(SMH10-071A) of SMH10-071A.wift

Max. $1.804 \mathrm{cps}$. $a=7.026702959713593700-004,10=7.511058300265564000-001$ (DuoSpray ())

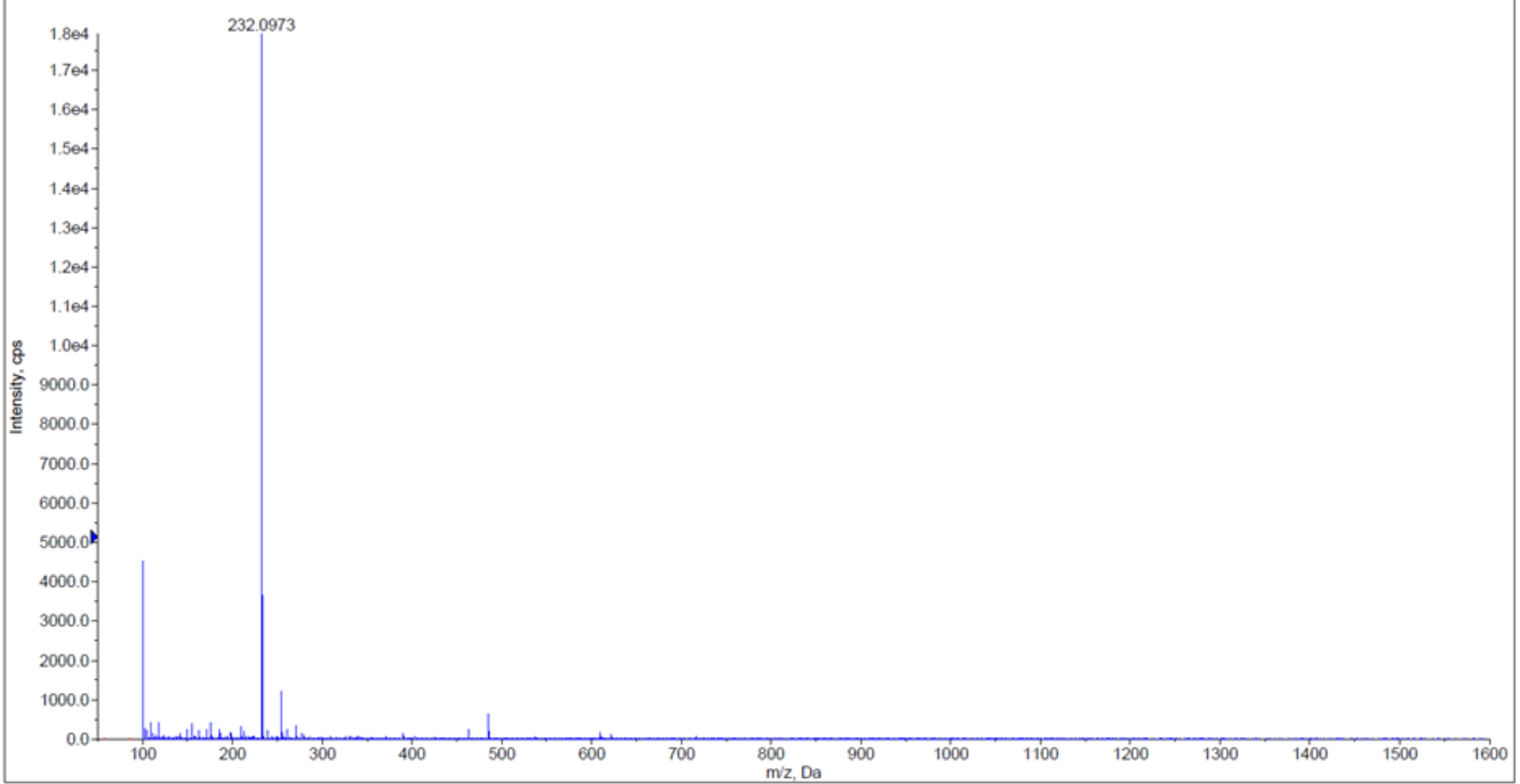

Figure S74: HRMS spectrum of 21. 
Chromatogram

SMH10-071A SMH10-071A.lcd

mAU

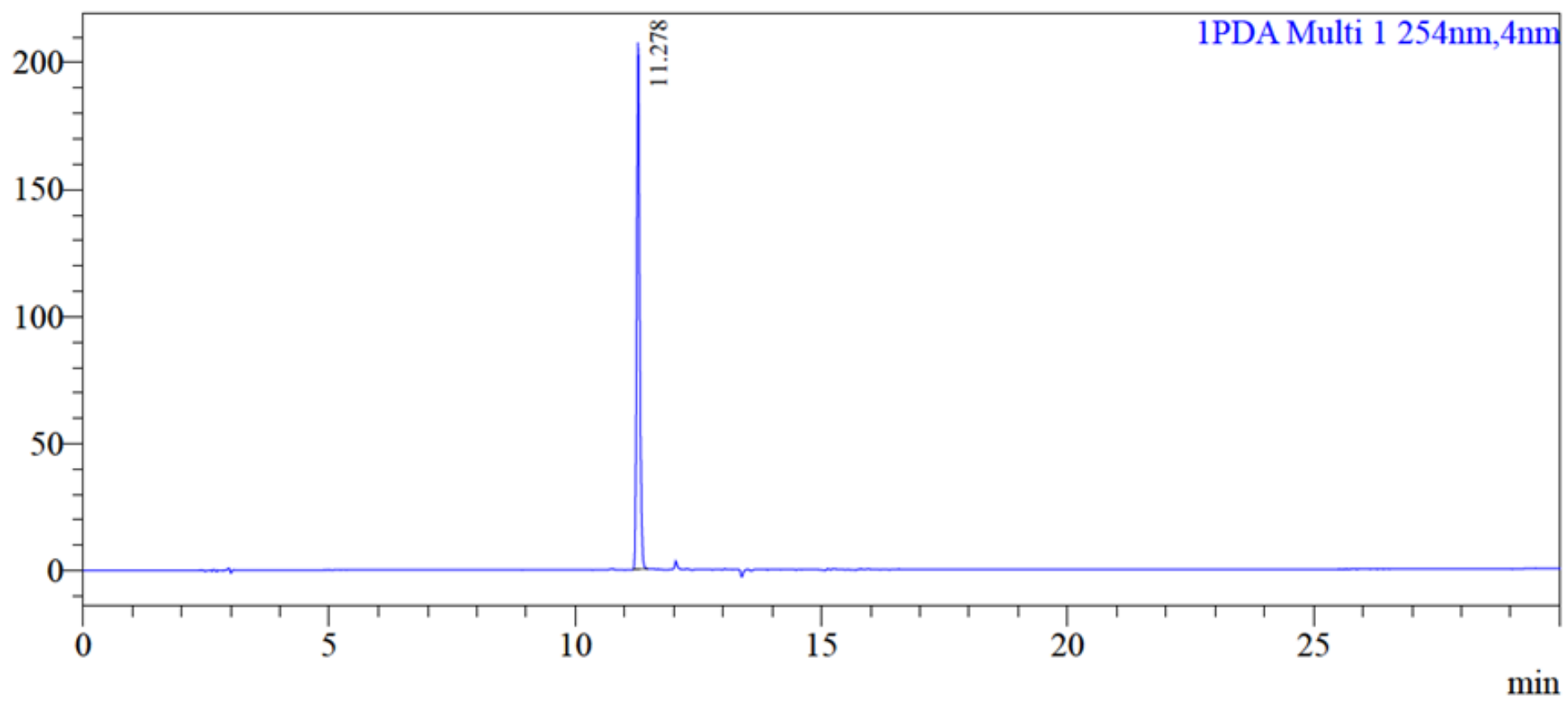

Peak Table

PDA Chl $254 \mathrm{~nm}$

\begin{tabular}{|r|r|r|r|r|r|}
\hline PDA Ceak\# & Ret. Time & Area & Height & Conc. & Name \\
\hline 1 & 11.278 & 898807 & 207242 & 100.000 & \\
\hline Total & & 898807 & 207242 & & \\
\hline
\end{tabular}

Figure S75: RP-HPLC trace of 21. 


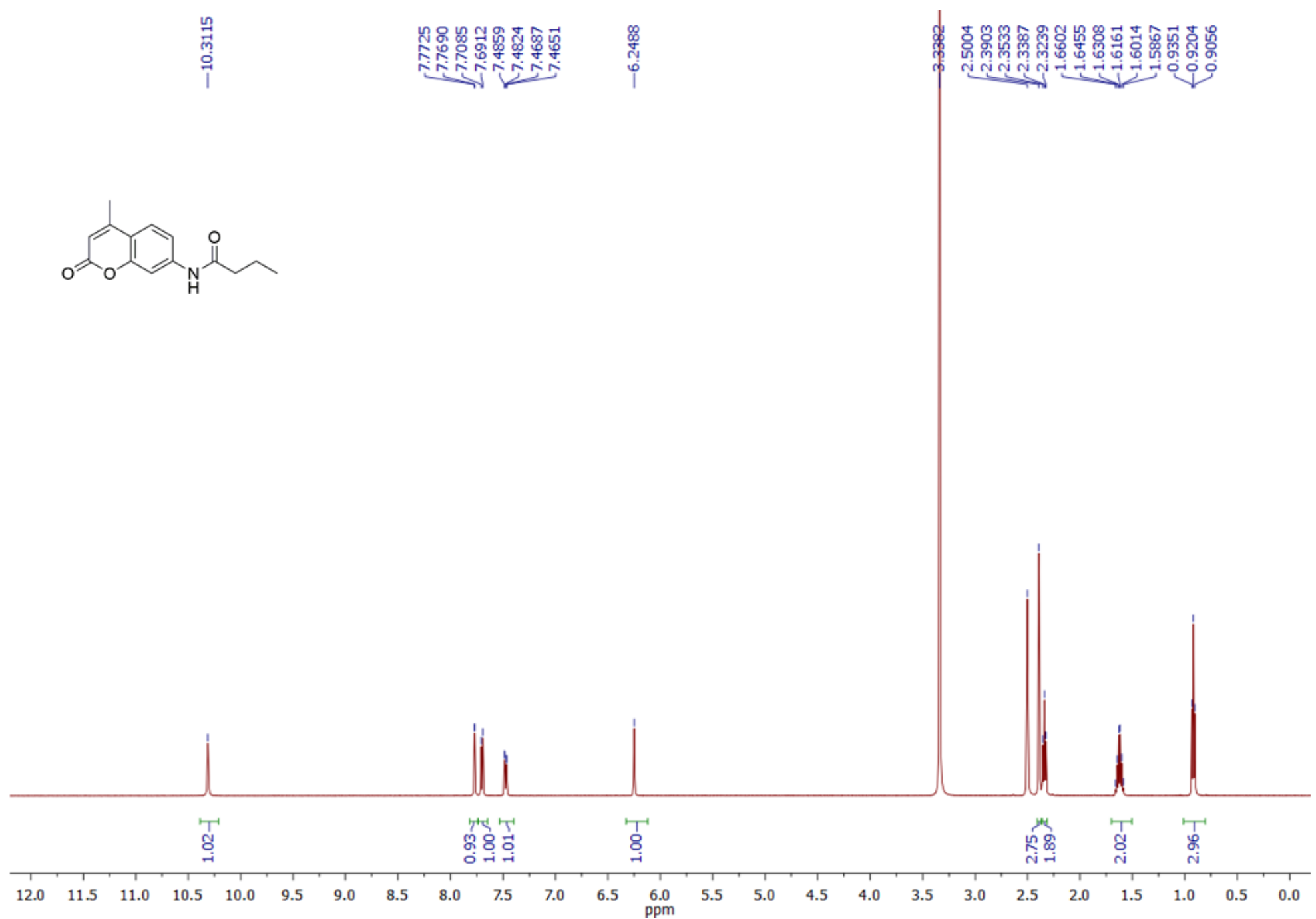

Figure S76: ${ }^{1} \mathrm{H}$ NMR $(500 \mathrm{MHz})$ spectrum of 22 in DMSO- $d_{6}$. 

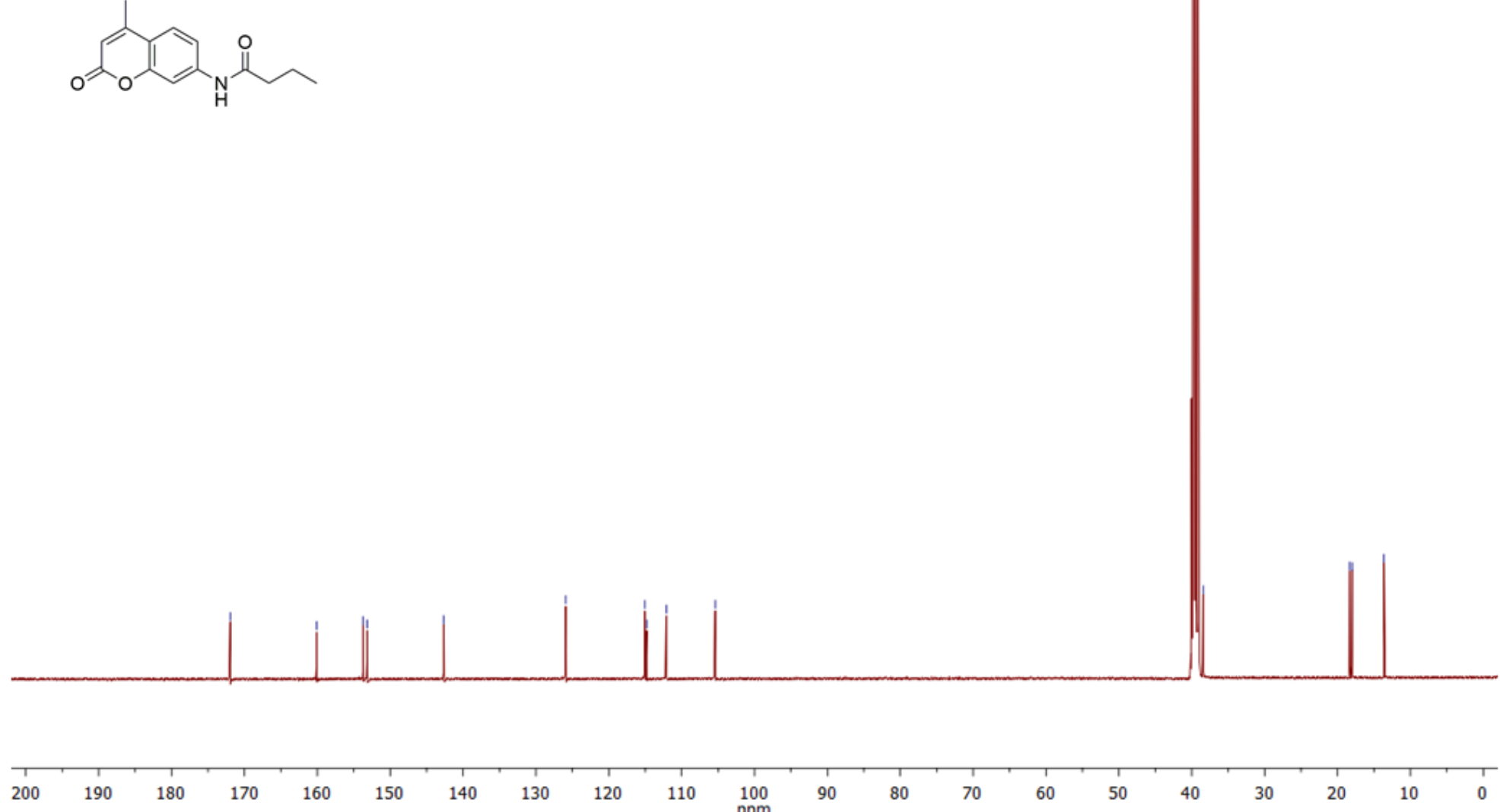

Figure S77: ${ }^{13} \mathrm{C}$ NMR (125 MHz) spectrum of 22 in DMSO- $d_{6}$ 

+TOF MS: Exp 1, 0.3008 to $0.3378 \mathrm{~min}$ from Sample 1 (SMH10-077A) of SMH10-077A.wiff
$\mathrm{a}=7.02675431123211500 \mathrm{e}-004,10=7.41725407137097230 \mathrm{e}-001$ (DuoSpray 0)

Max. $3.604 \mathrm{cps}$.

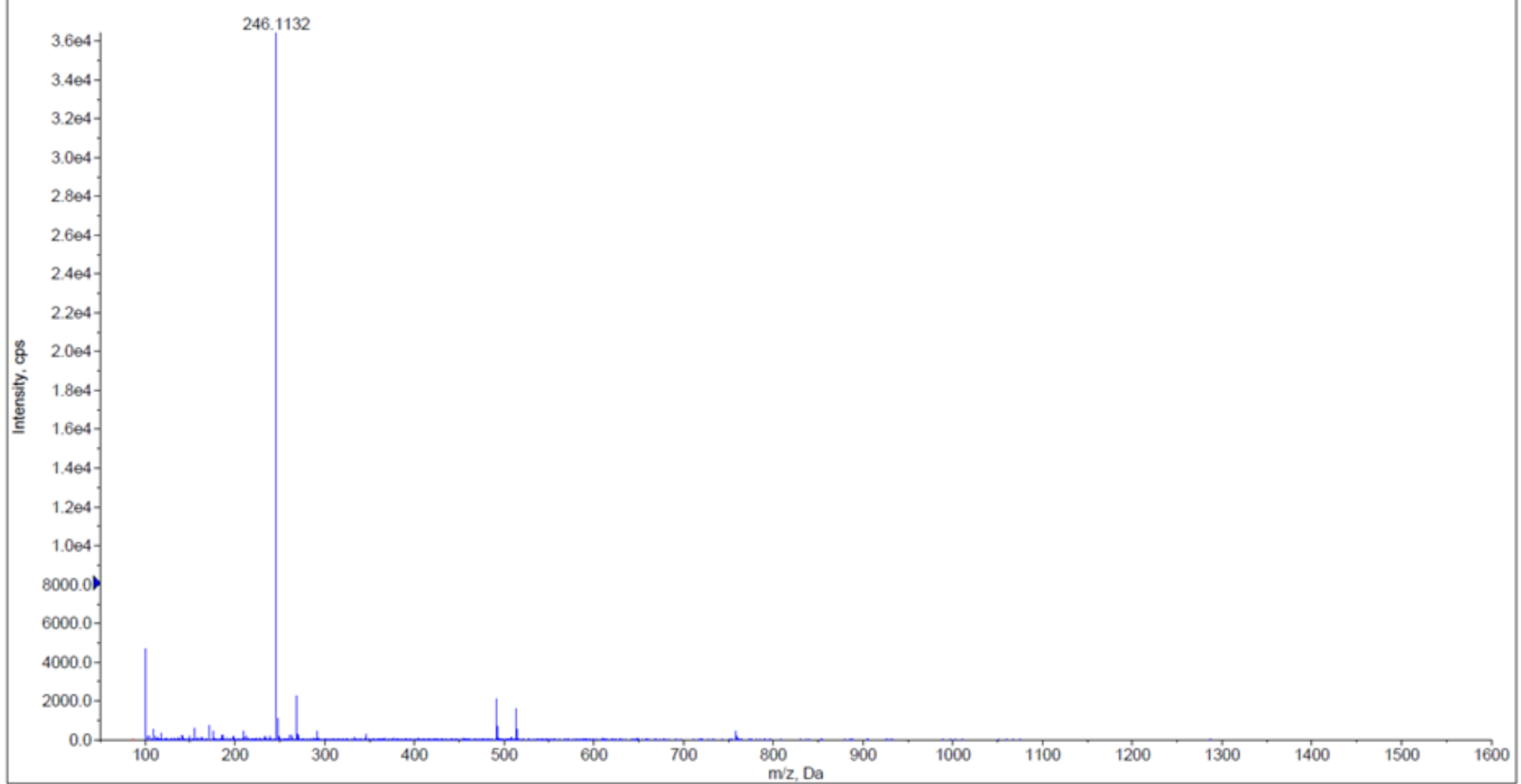

Figure S78: HRMS spectrum of 22. 
Chromatogram

mAU

SMH10-077A SMH10-077A.led

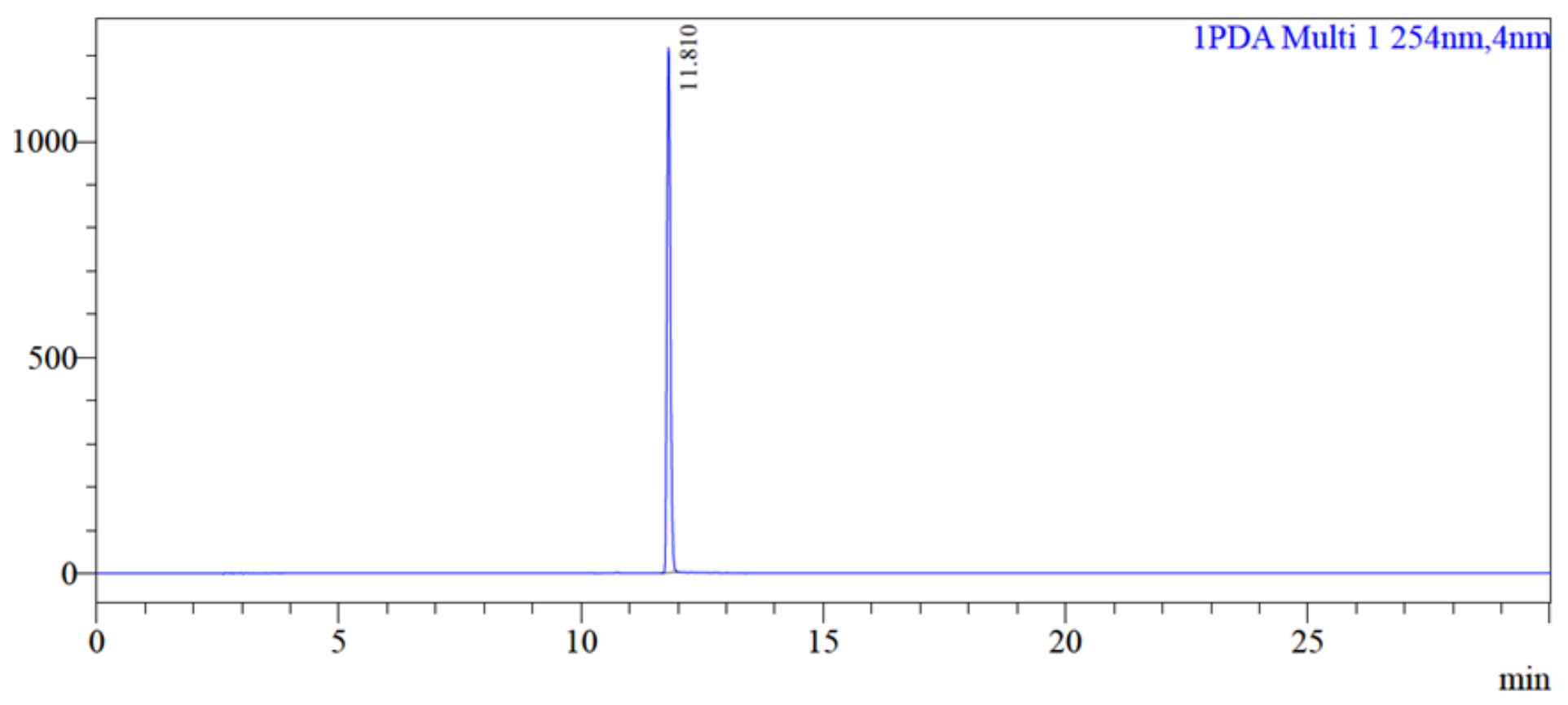

Peak Table

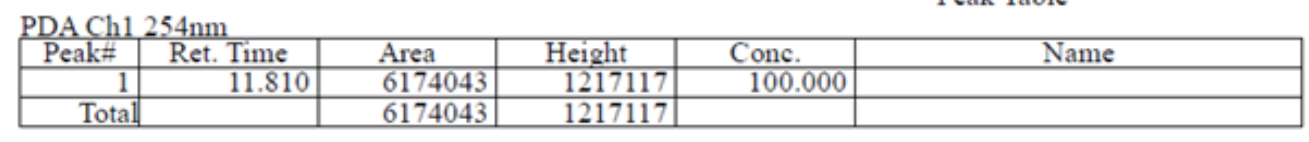

Figure S79: RP-HPLC trace of 22. 


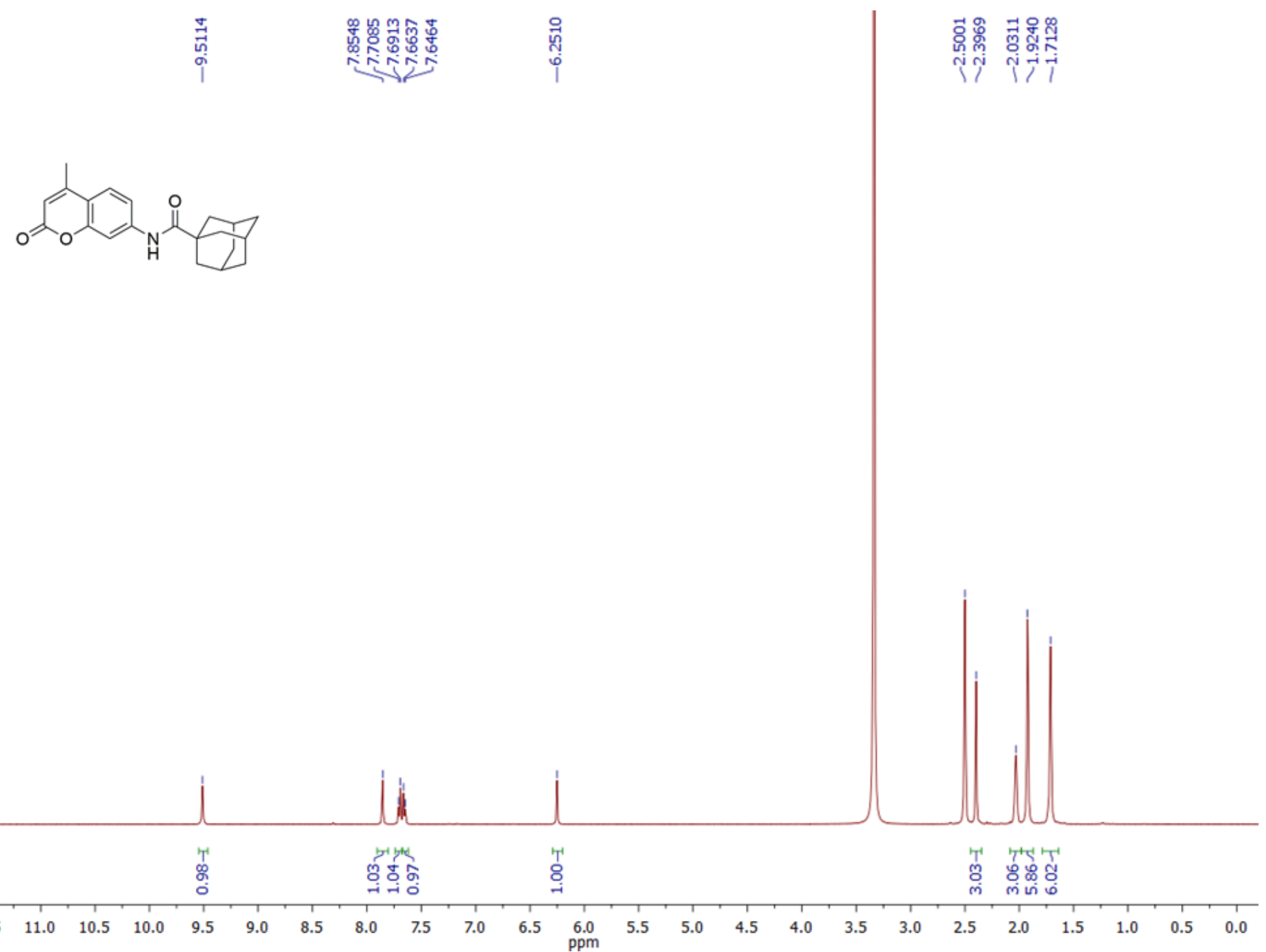

Figure S80: ${ }^{1} \mathrm{H}$ NMR $(500 \mathrm{MHz})$ spectrum of 23 in DMSO- $d_{6}$. 

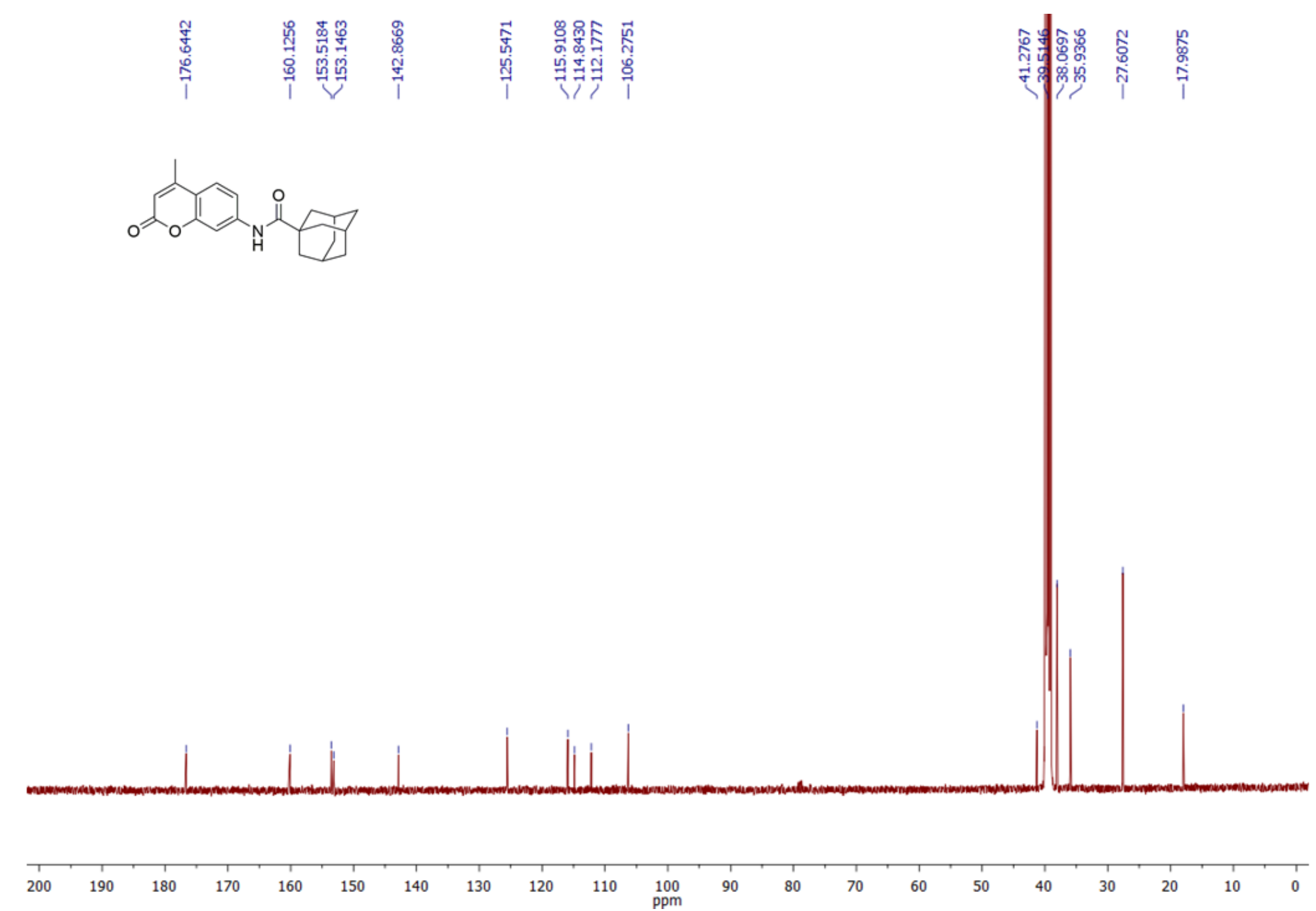

Figure S81: ${ }^{13} \mathrm{C}$ NMR $(125 \mathrm{MHz})$ spectrum of 23 in DMSO- $d_{6}$. 
+TOF MS: 0.1344 to $0.1437 \mathrm{~min}$ from Sample 5 (SON04-091C) of SON04-091. wit

a= $=7.026787697743122000-004,10=9.399056016771610800-001$ (DuoSpray ())

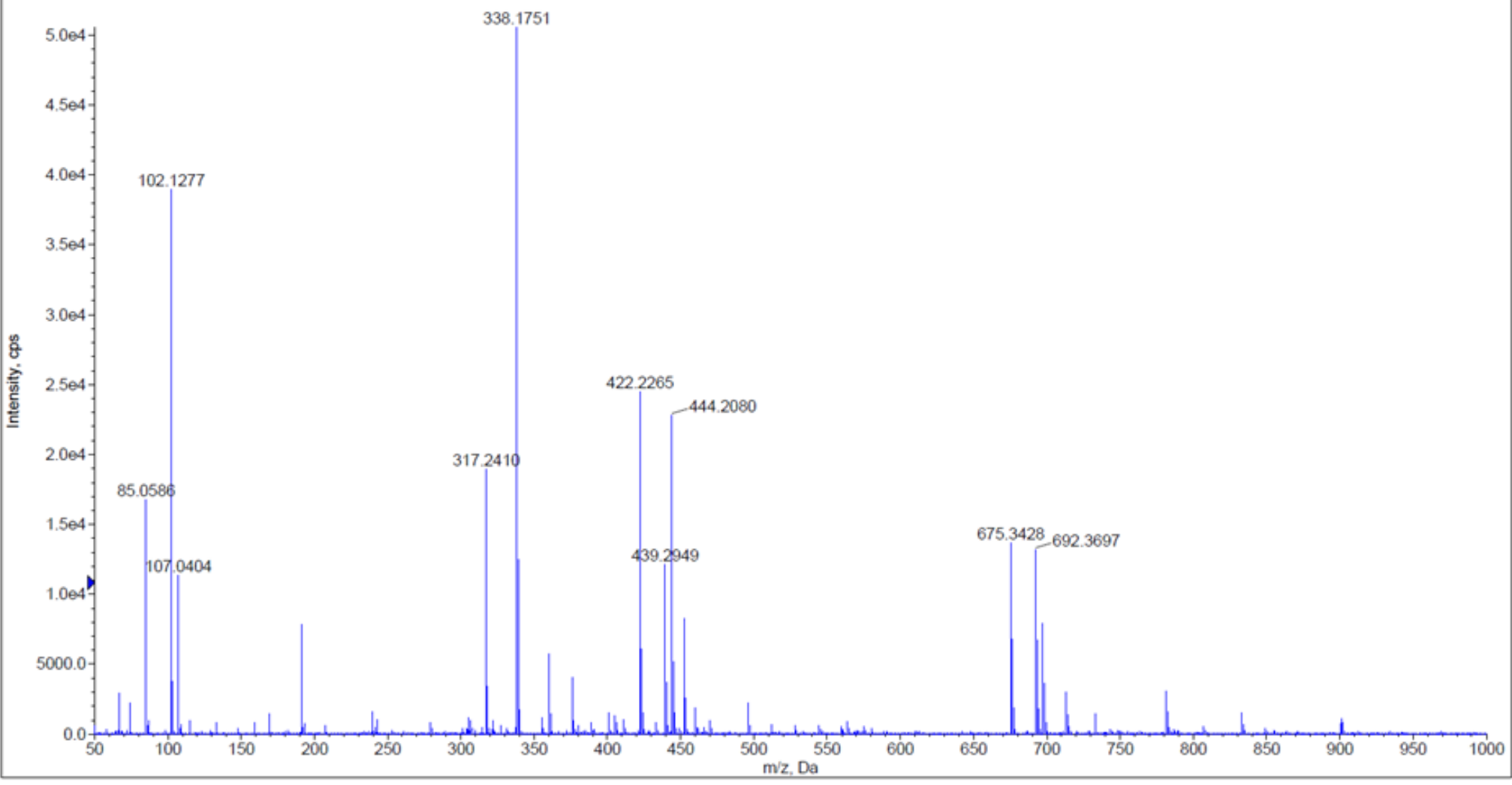

Figure S82: HRMS spectrum for 23. 

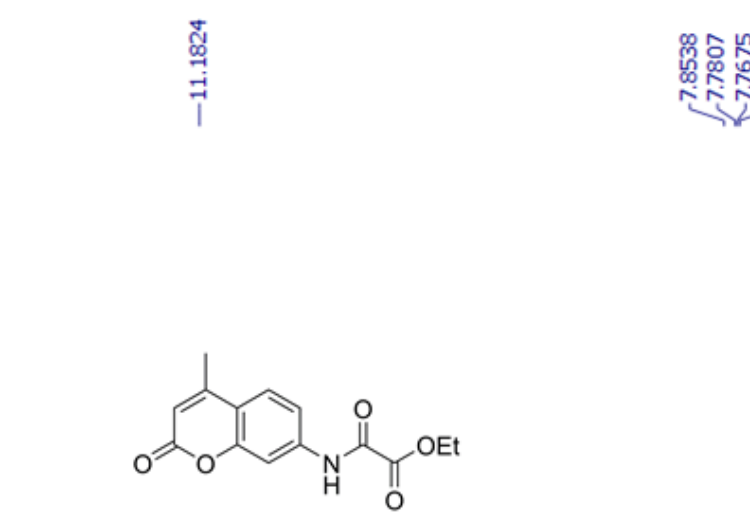

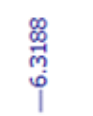
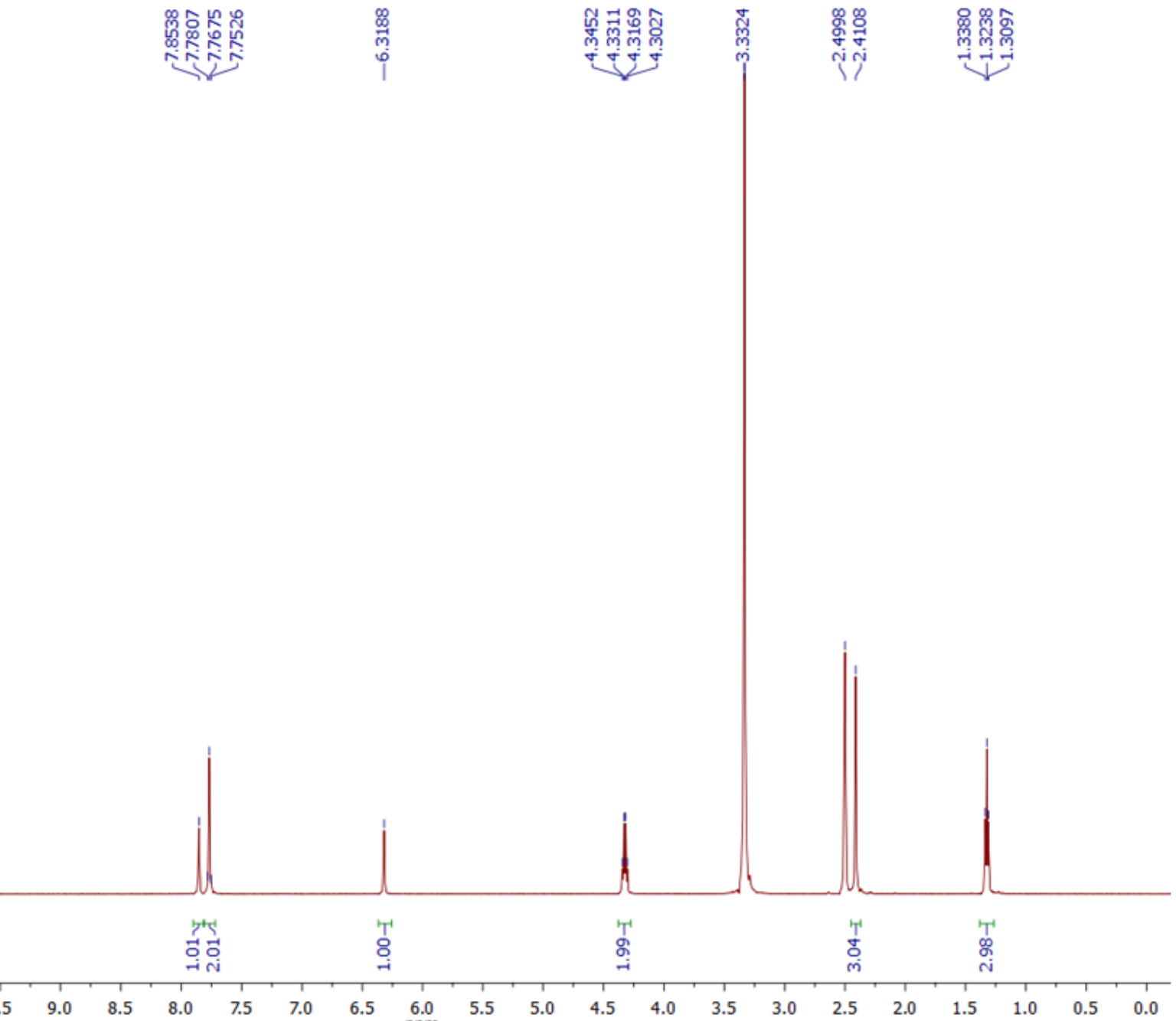

Figure S83: ${ }^{1} \mathrm{H}$ NMR $(500 \mathrm{MHz})$ spectrum of 24 in DMSO- $d_{6}$. 


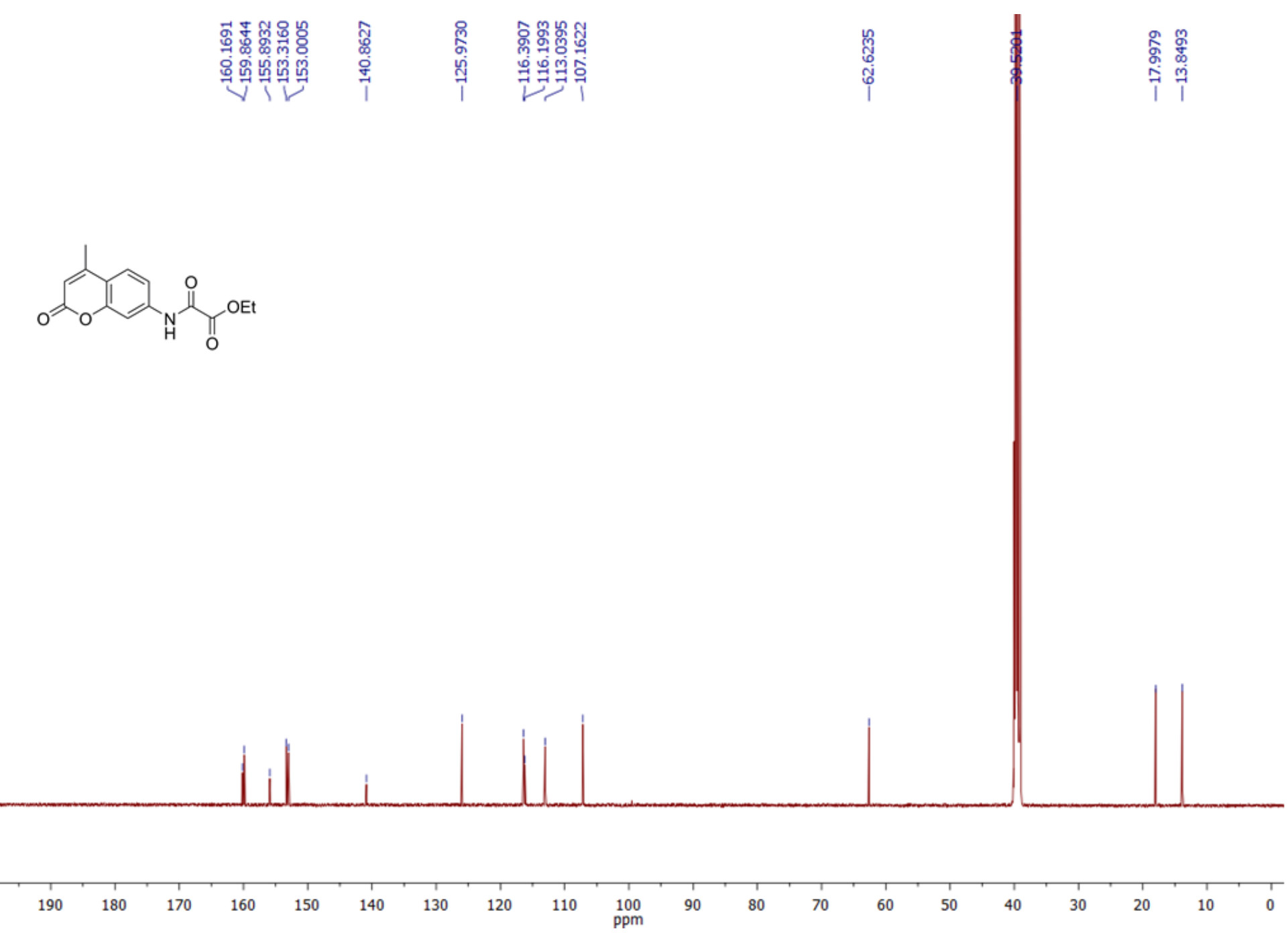

Figure S84: ${ }^{13} \mathrm{C}$ NMR (125 MHz) spectrum of 24 in DMSO- $d_{6}$. 
+TOF MS: Exp 1, 0.0783 to $0.0876 \mathrm{~min}$ from Sample 1(SMH12-077A) of SMH12-077A.wiff
$a=7.02678800778209780 \mathrm{e}-004,10=8.37267332804711110 \mathrm{e}-001$ (DuoSpray 0 )

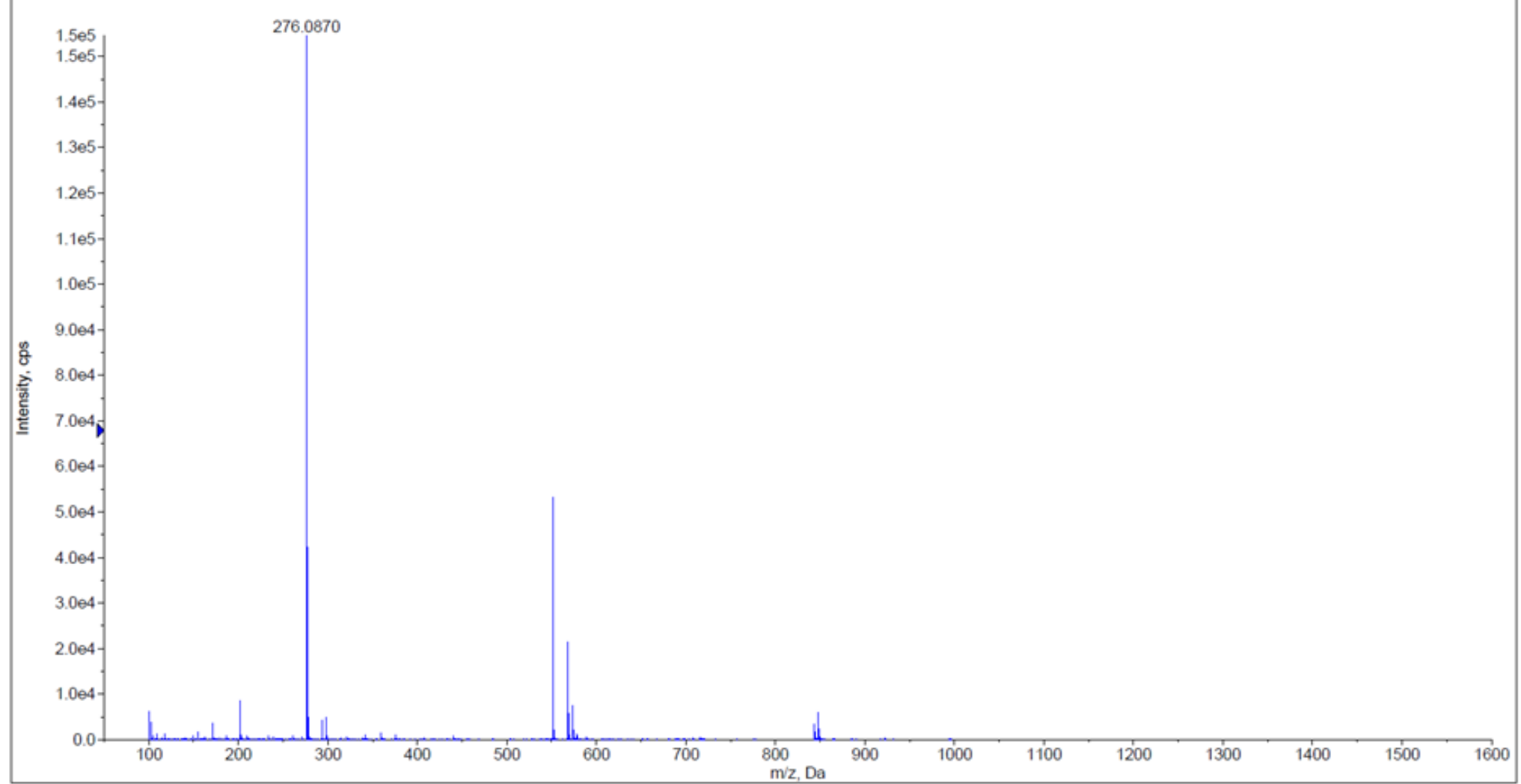

Figure S85: HRMS spectrum of $\mathbf{2 4}$. 
Chromatogram

mAU

SMH12-077A SMH12-077A.lcd

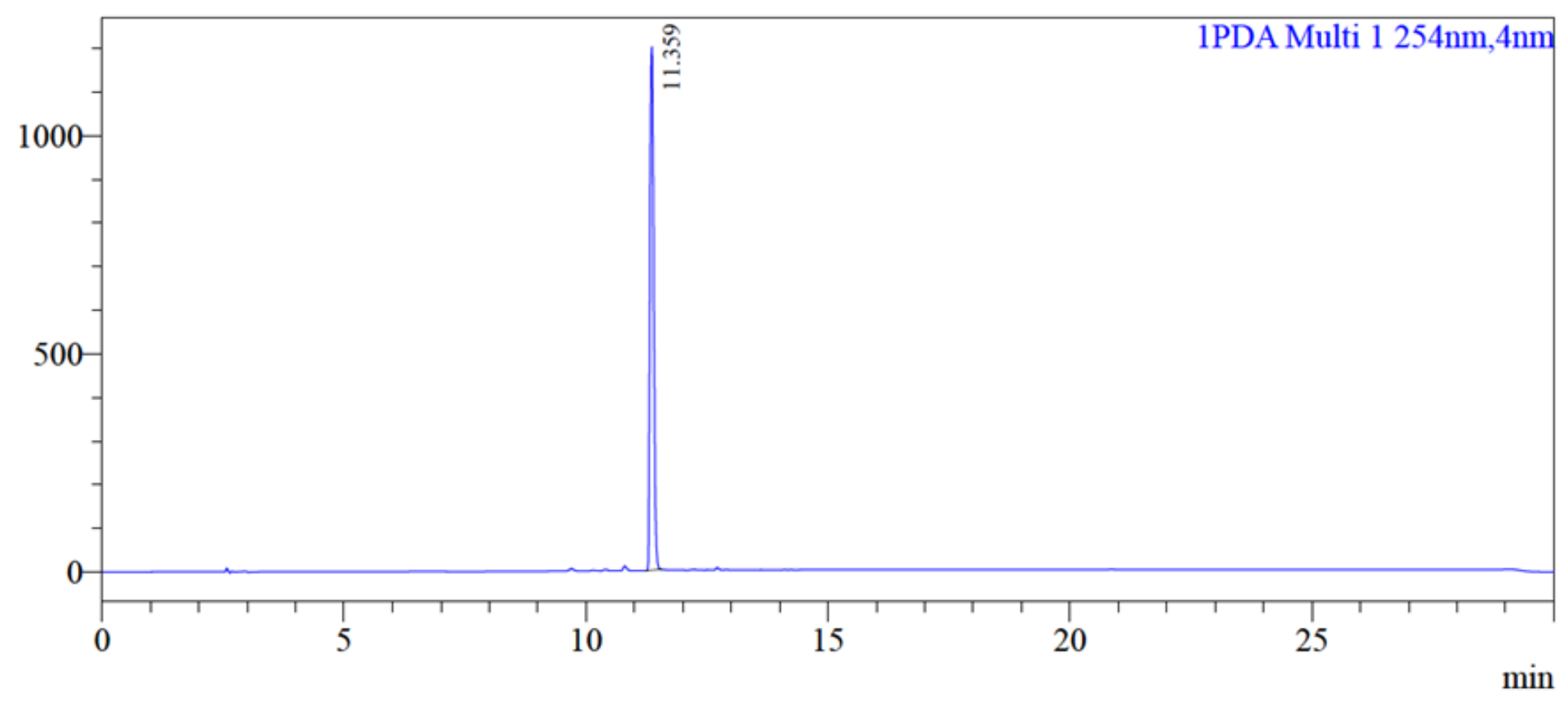

Peak Table

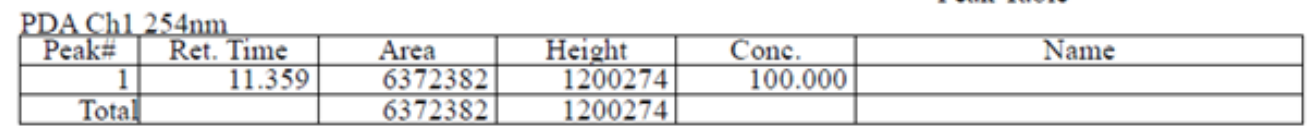

Figure S86: RP-HPLC trace of 24. 


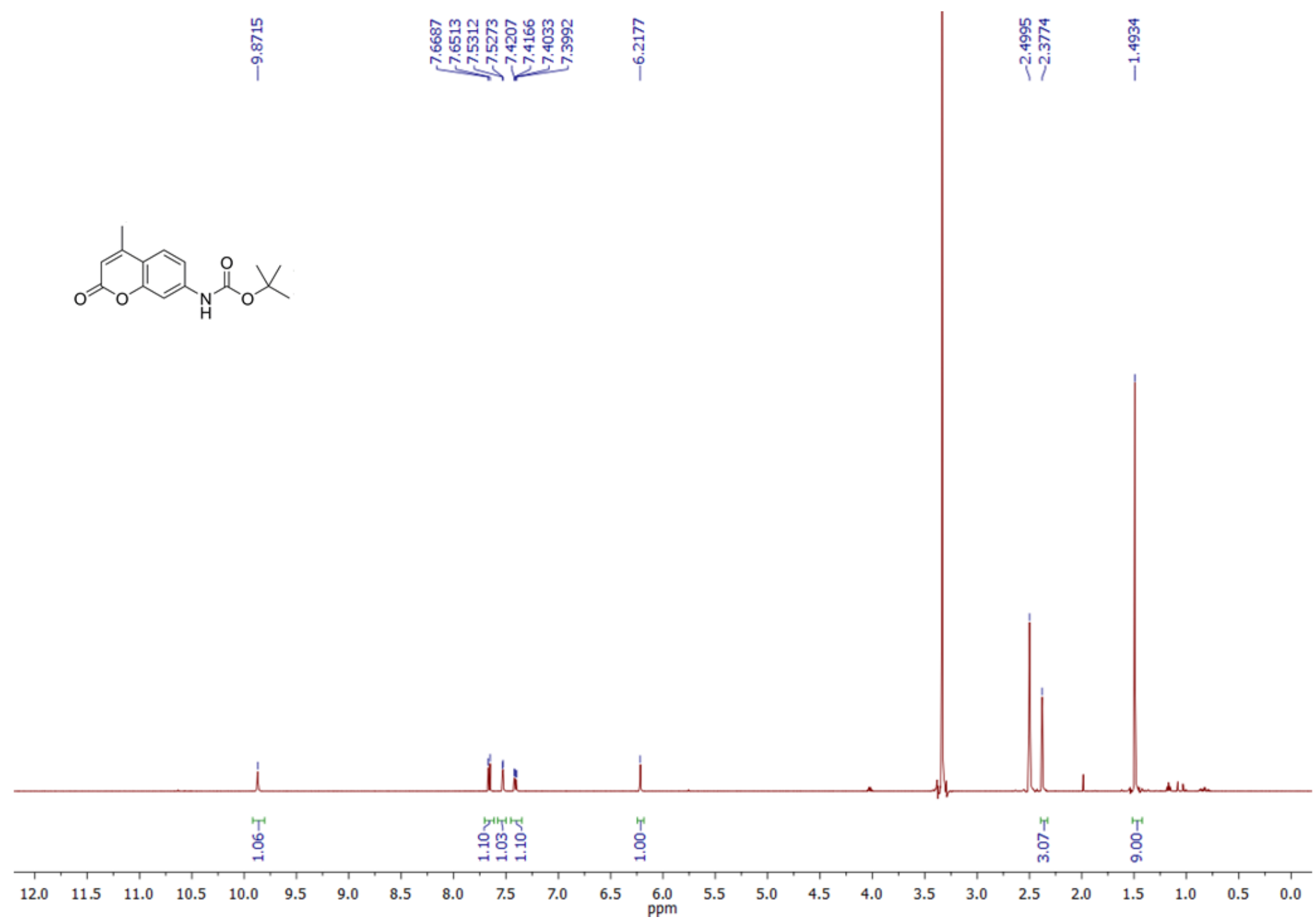

Figure S87: ${ }^{1} \mathrm{H}$ NMR (500 MHz) spectrum of 25 in DMSO- $d_{6}$. 


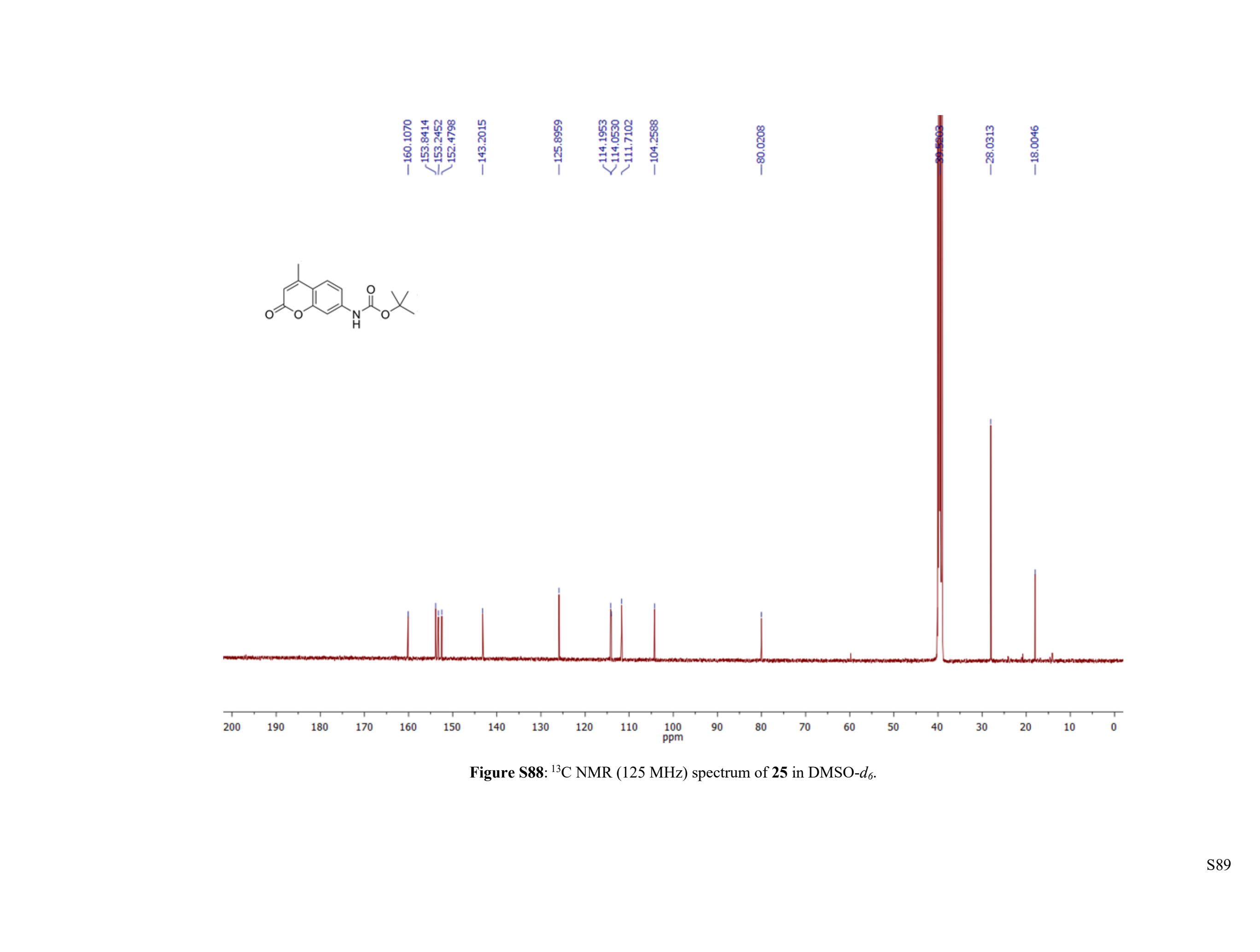


- +TOF MS: Exp 1, 0.3934 to 0.4212 min from Sample 1 (SMH10-0998) of SMH10-099B.wift different calibrations (DuoSpray ())

Max. $7129.5 \mathrm{cps}$.

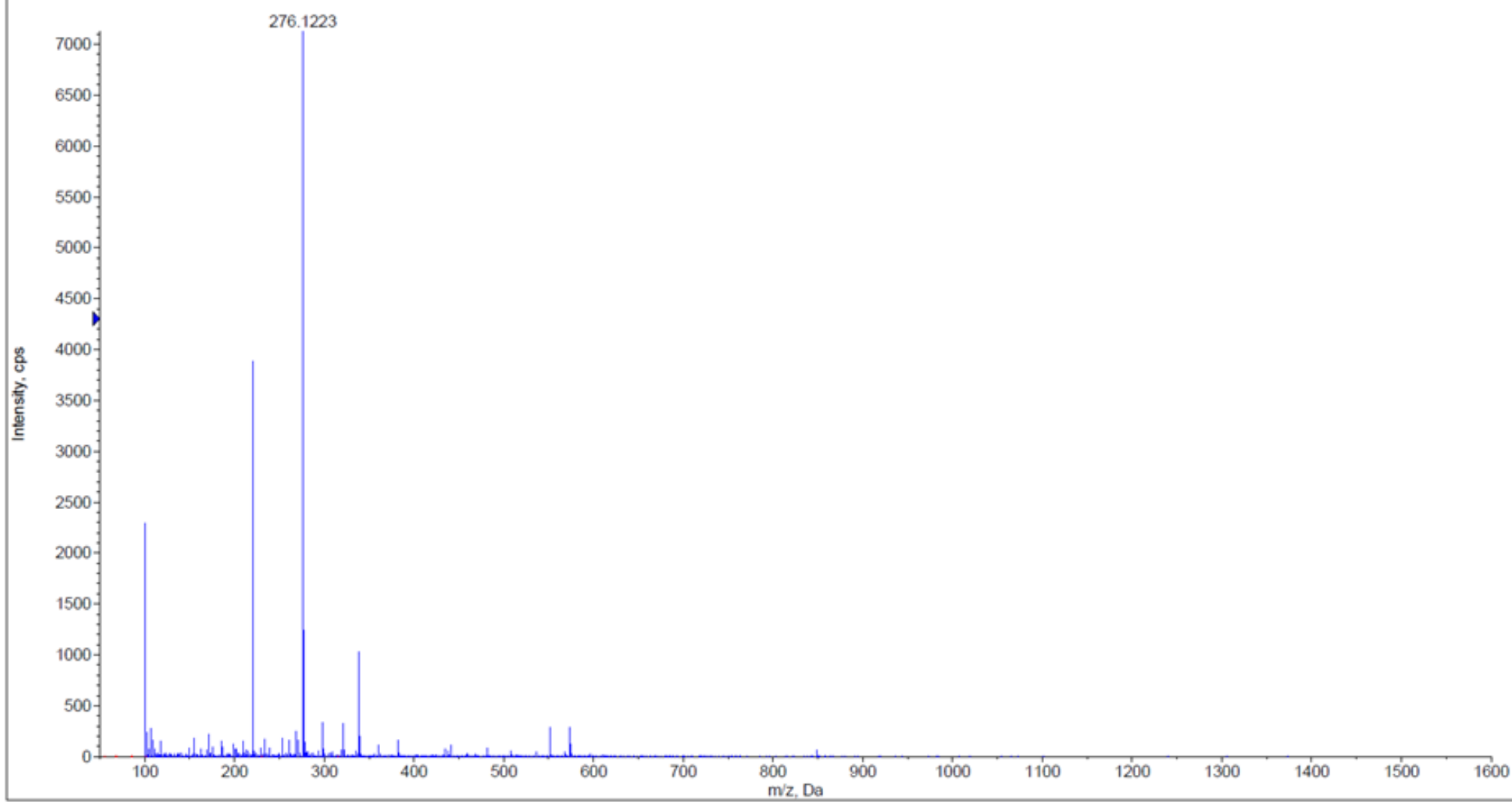

Figure S89: HRMS spectrum of 25. 
Chromatogram

SMH10-099B SMH10-099B.led

mAU

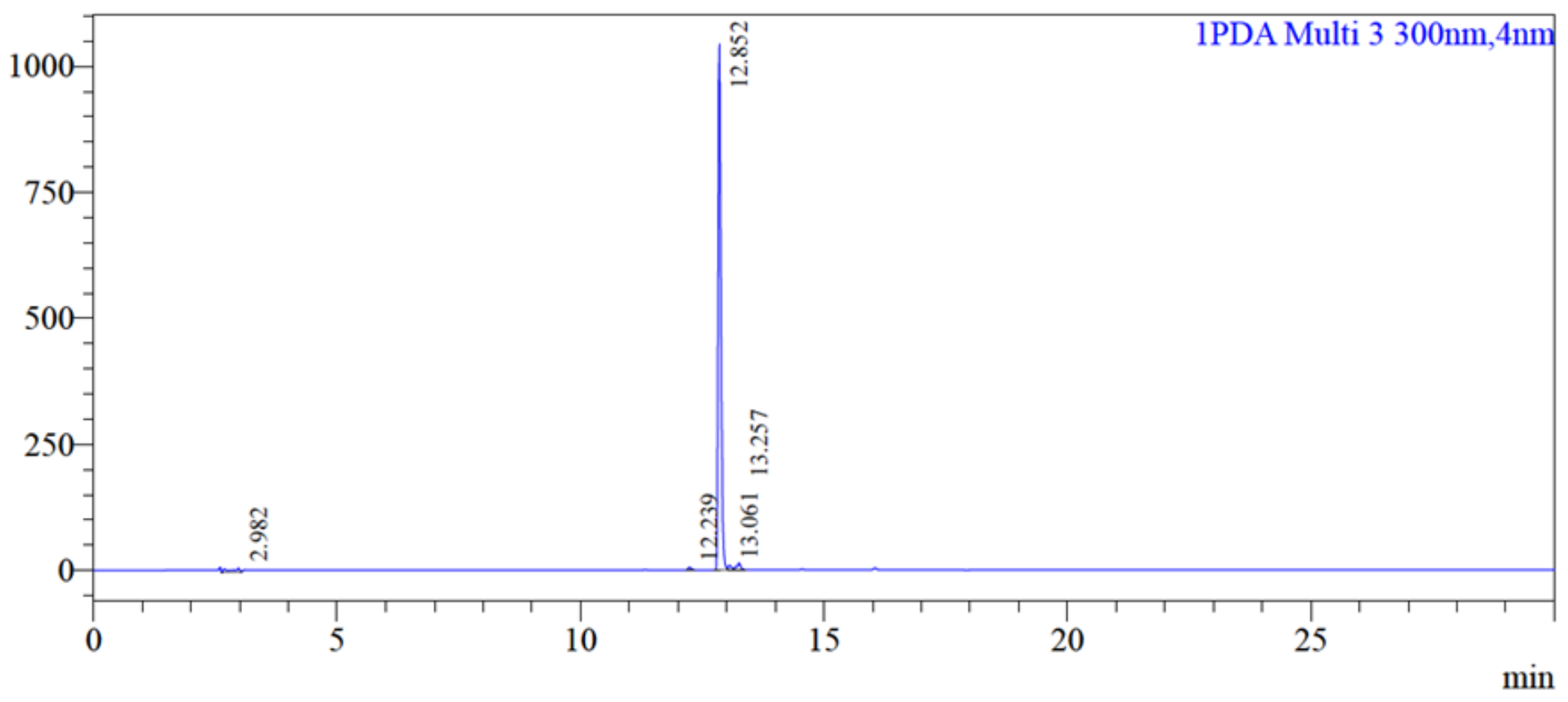

Peak Table

PDA Ch3 $300 \mathrm{~nm}$
\begin{tabular}{|r|r|r|r|r|l|}
\hline Peak\# & Ret. Time & \multicolumn{1}{|c|}{ Area } & Height & \multicolumn{1}{c|}{ Conc. } & \\
\hline 1 & 2.982 & 91409 & 7478 & 2.085 & \\
\hline 2 & 12.239 & 22249 & 5539 & 0.507 & \\
\hline 3 & 12.852 & 4156727 & 1044023 & 94.809 & \\
\hline 4 & 13.061 & 45032 & 8957 & 1.027 & \\
\hline 5 & 13.257 & 68912 & 12823 & 1.572 & \\
\hline Total & & 4384330 & 1078819 & & \\
\hline
\end{tabular}

Figure S90: RP-HPLC trace of $\mathbf{2 5 .}$ 


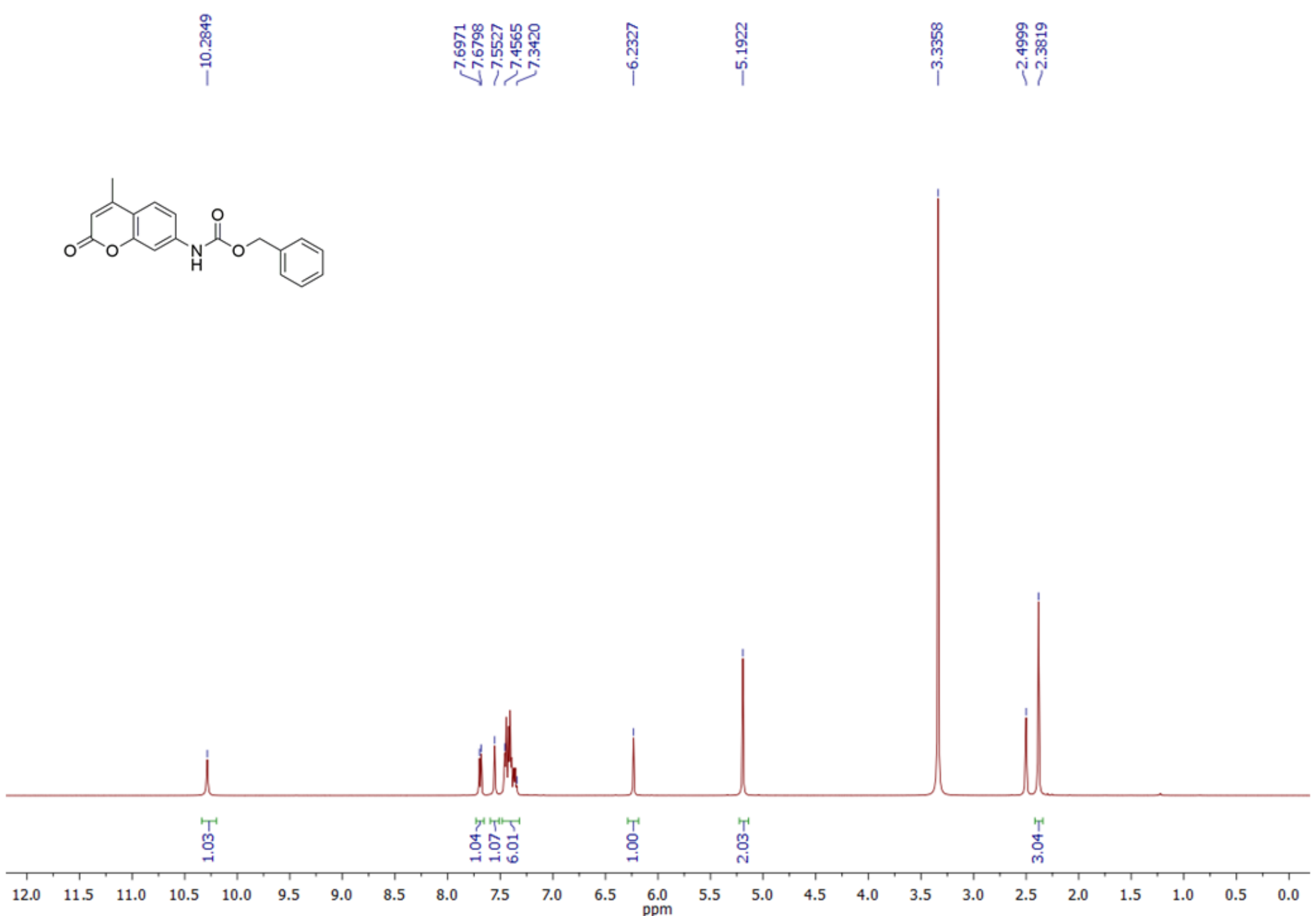

Figure S91: ${ }^{1} \mathrm{H}$ NMR (500 MHz) spectrum of 26 in DMSO- $d_{6}$. 


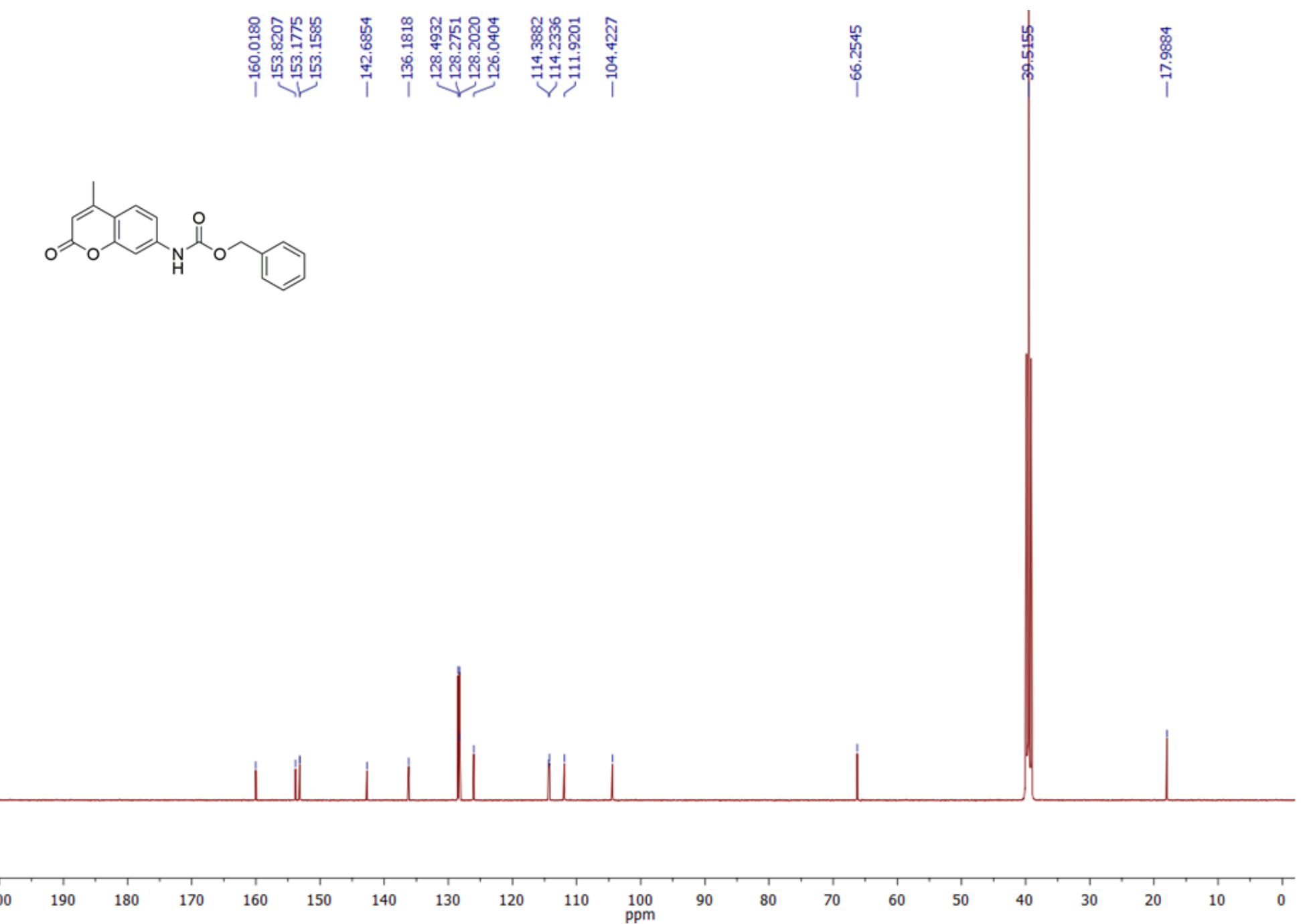

Figure S92: ${ }^{13} \mathrm{C}$ NMR (125 MHz) spectrum of 26 in DMSO- $d_{6}$ 
+TOF MS: 0.0832 to $0.0879 \mathrm{~min}$ from Sample 3 (SON04-031A) of SON04-031.

$\operatorname{Max} 8.004 \mathrm{cps}$.

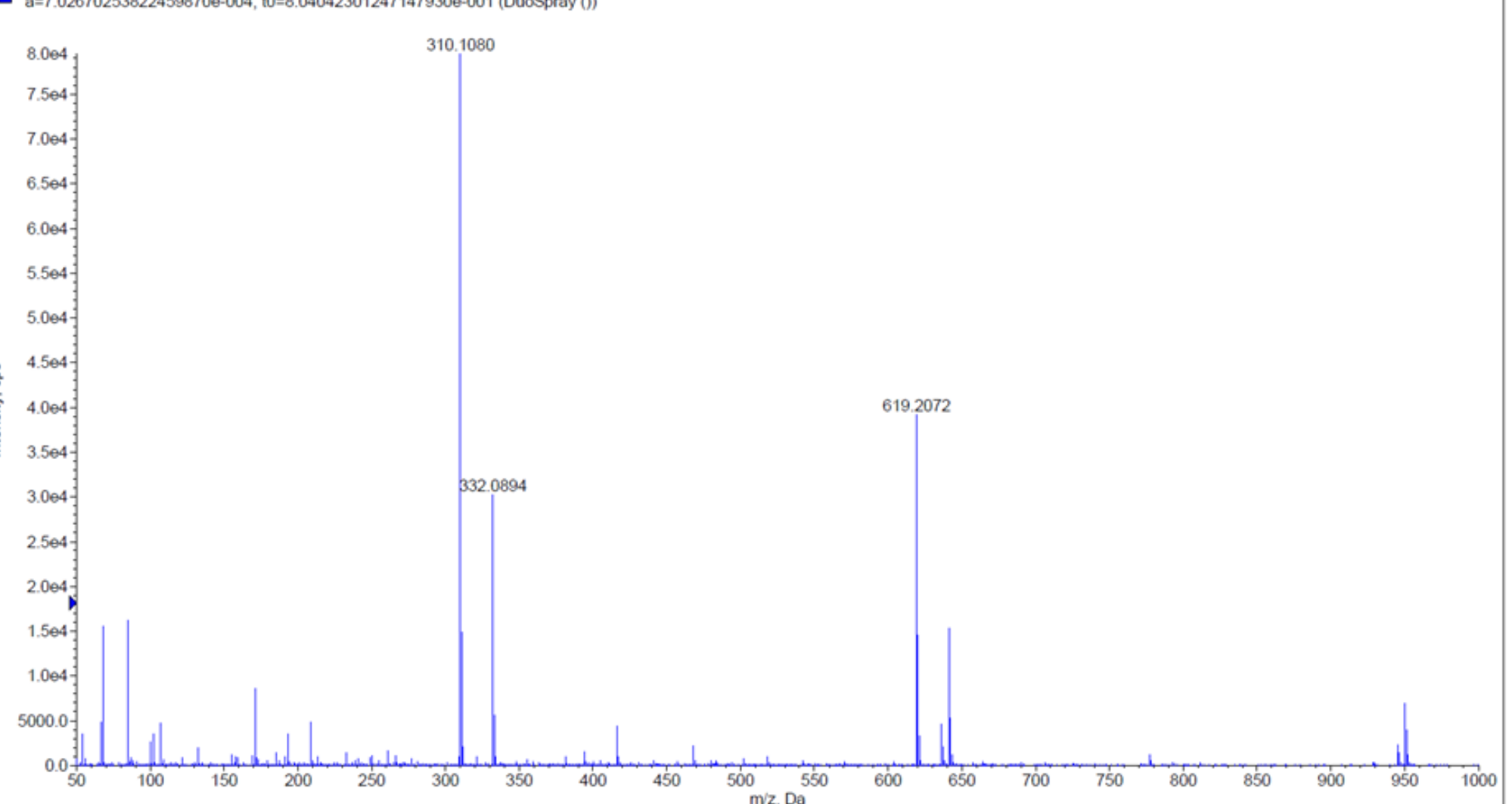

Figure S93: HRMS spectrum for 26. 
Chromatogram

SON04-031A SON04-031A.led

mAU

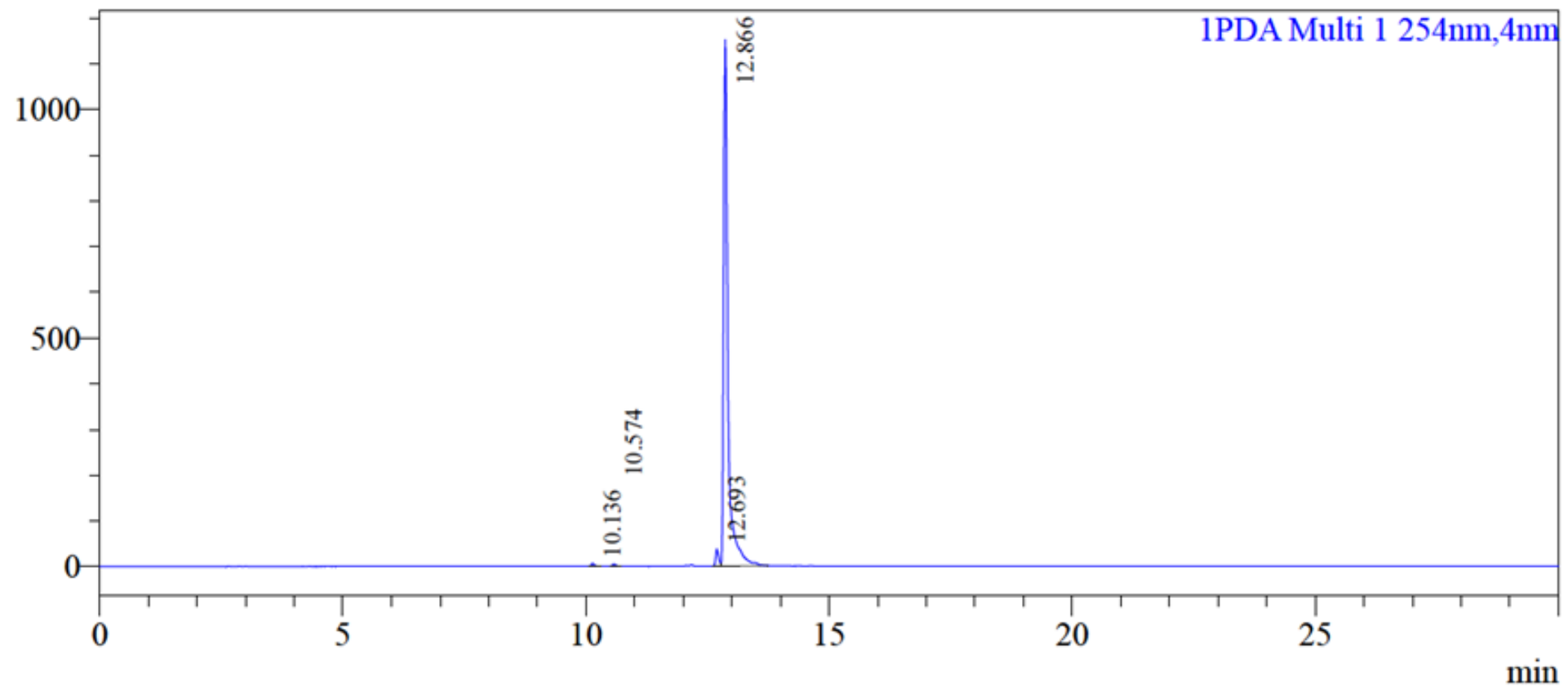

Peak Table

PDA Chl $254 \mathrm{~nm}$
\begin{tabular}{|r|r|r|r|r|r|}
\hline Peak= & Ret. Time & \multicolumn{1}{c|}{ Area } & Height & Conc. & Name \\
\hline 1 & 10.136 & 28155 & 6197 & 0.382 & \\
\hline 2 & 10.574 & 20379 & 4866 & 0.276 & \\
\hline 3 & 12.693 & 160073 & 36571 & 2.171 & \\
\hline 4 & 12.866 & 7162942 & 1152490 & 97.170 & \\
\hline Total & & 7371549 & 1200124 & & \\
\hline
\end{tabular}

Figure S94: RP-HPLC trace for $\mathbf{2 6}$ 


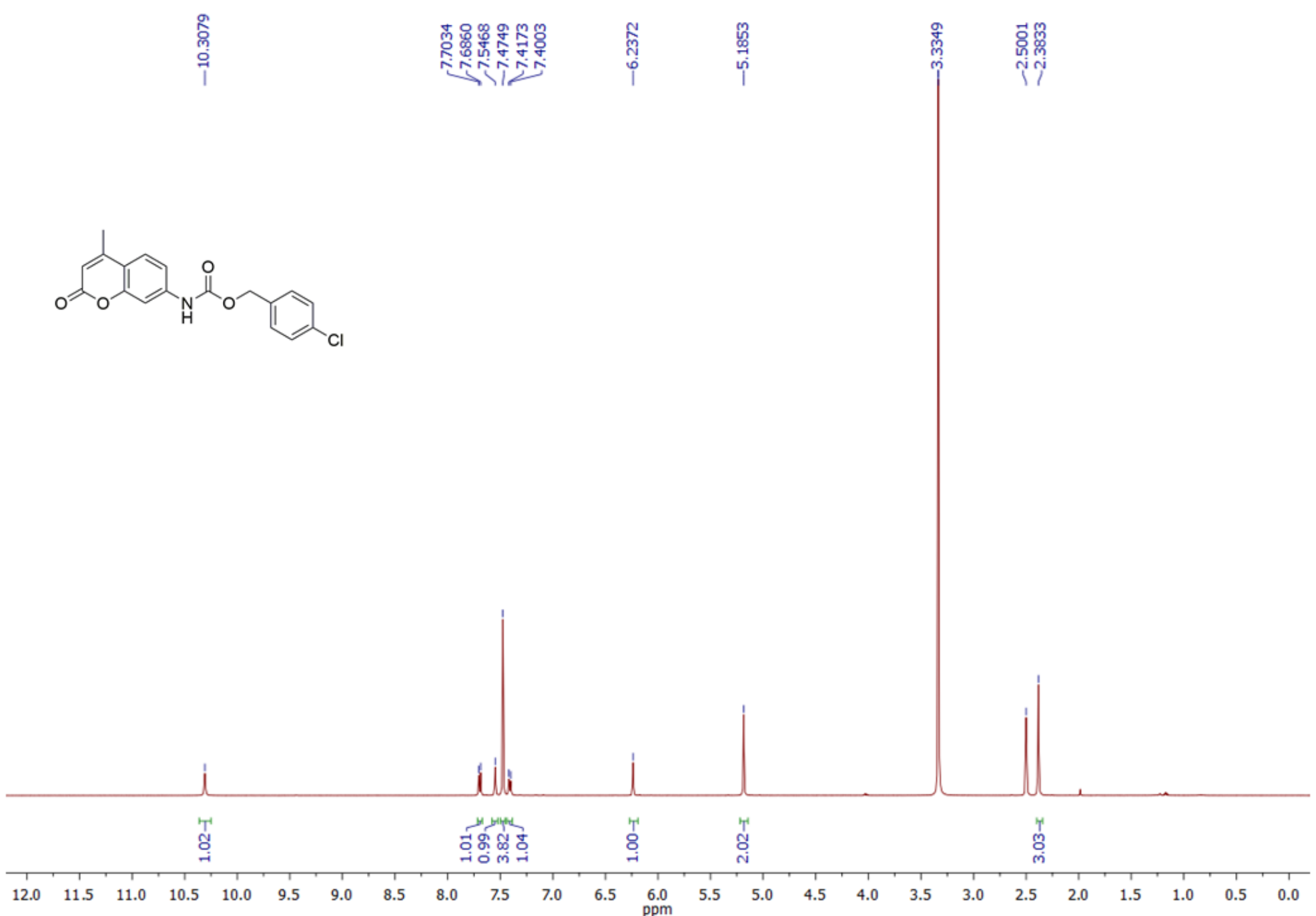

Figure S95: ${ }^{1} \mathrm{H}$ NMR $(500 \mathrm{MHz})$ spectrum of 27 in DMSO- $d_{6}$. 


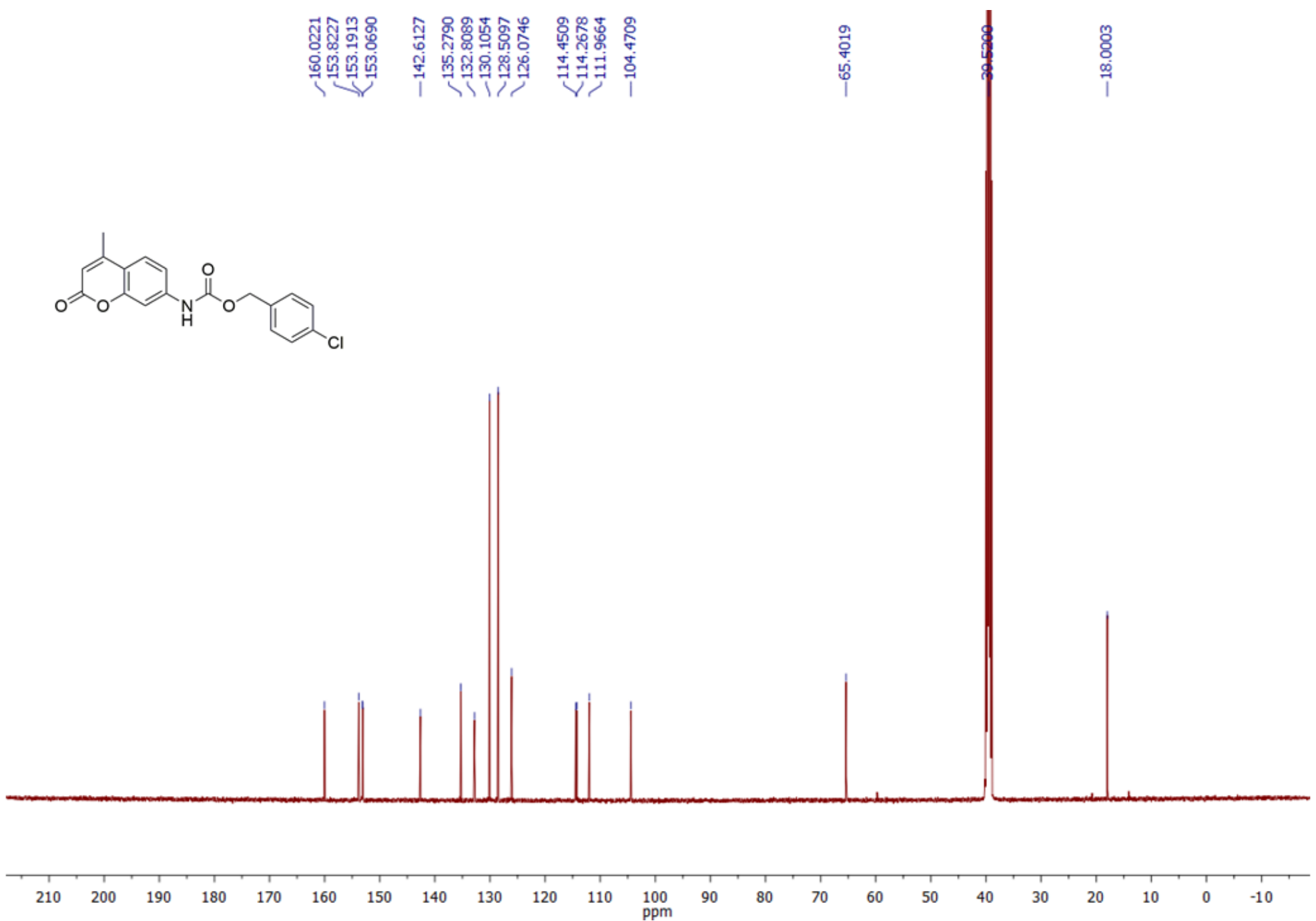

Figure S96: ${ }^{13} \mathrm{C}$ NMR (125 MHz) spectrum of 27 in DMSO- $d_{6}$ 
+TOF MS: Exp 1, 0.1154 to 0.1247 min from Sample 1 (SMH12-117C) of SMH12-117C.wiff

Max. 2.1e4 cps. $\mathrm{a}=7.02678387963701300 \mathrm{e}-004,10=8.687775018092575000-001$ (DuoSpray (0)

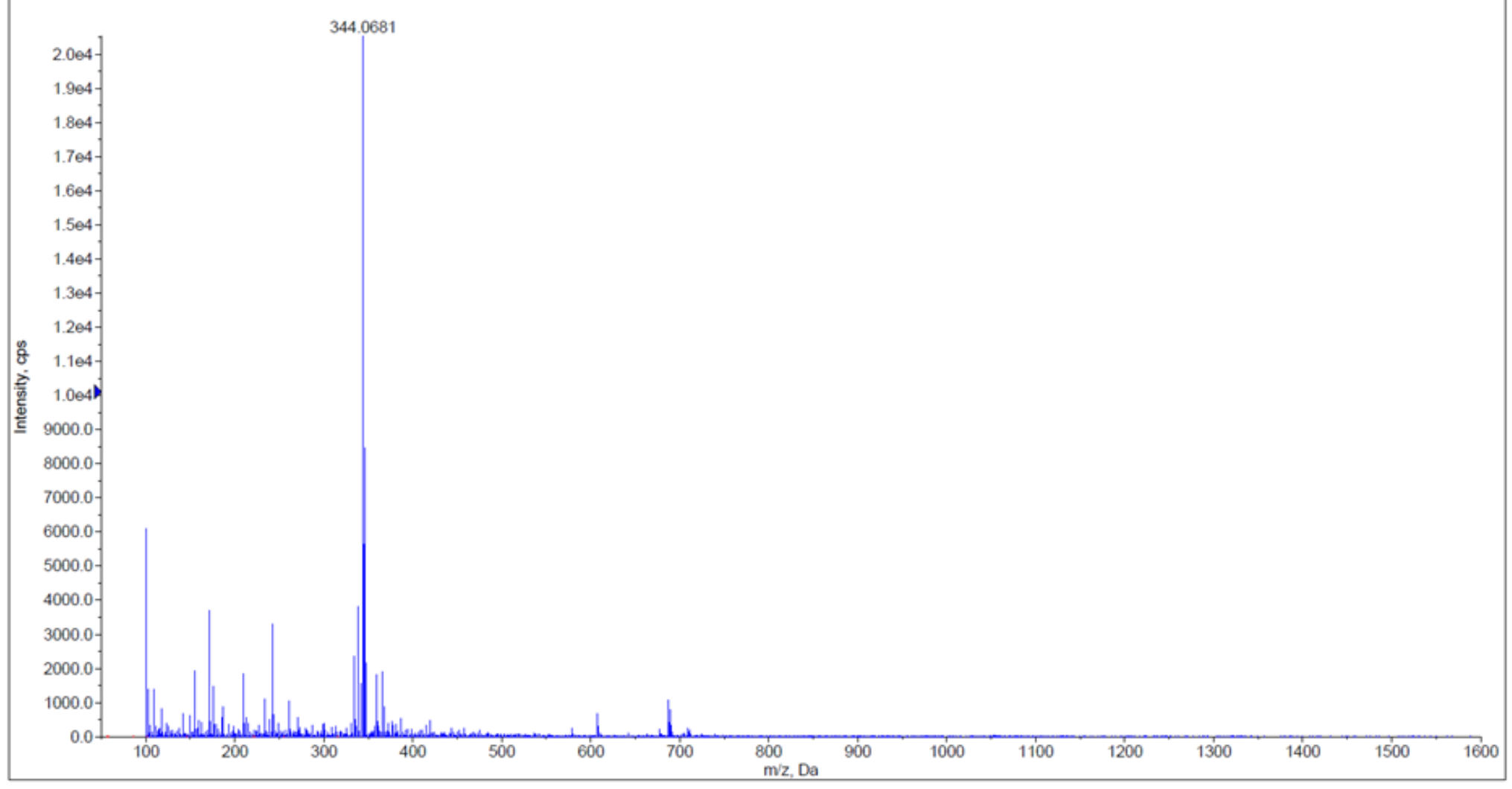

Figure S97: HRMS spectrum of 27. 
Chromatogram

SMH12-117C SMH12-117C.lcd

mAU

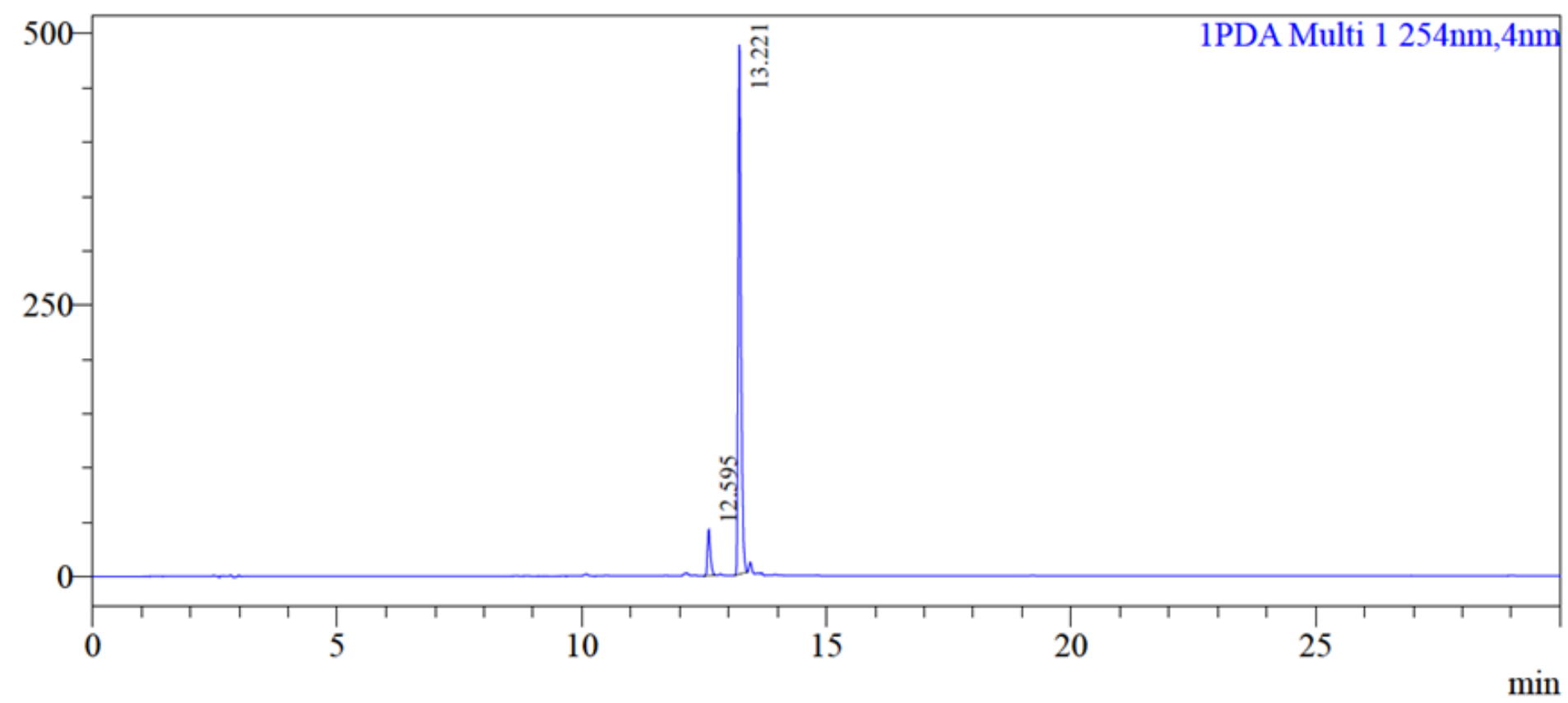

Peak Table

PDA Ch1 $254 \mathrm{~nm}$
\begin{tabular}{|r|r|r|r|r|r|}
\hline Peak\# & Ret. Time & \multicolumn{1}{|c|}{ Area } & \multicolumn{1}{c|}{ Height } & \multicolumn{1}{c|}{ Conc. } & Name \\
\hline 1 & 12.595 & 176025 & 42229 & 8.098 & \\
\hline 2 & 13.221 & 1997666 & 487422 & 91.902 & \\
\hline Total & & 2173691 & 529651 & & \\
\hline
\end{tabular}

Figure S98: RP-HPLC trace of 27. 


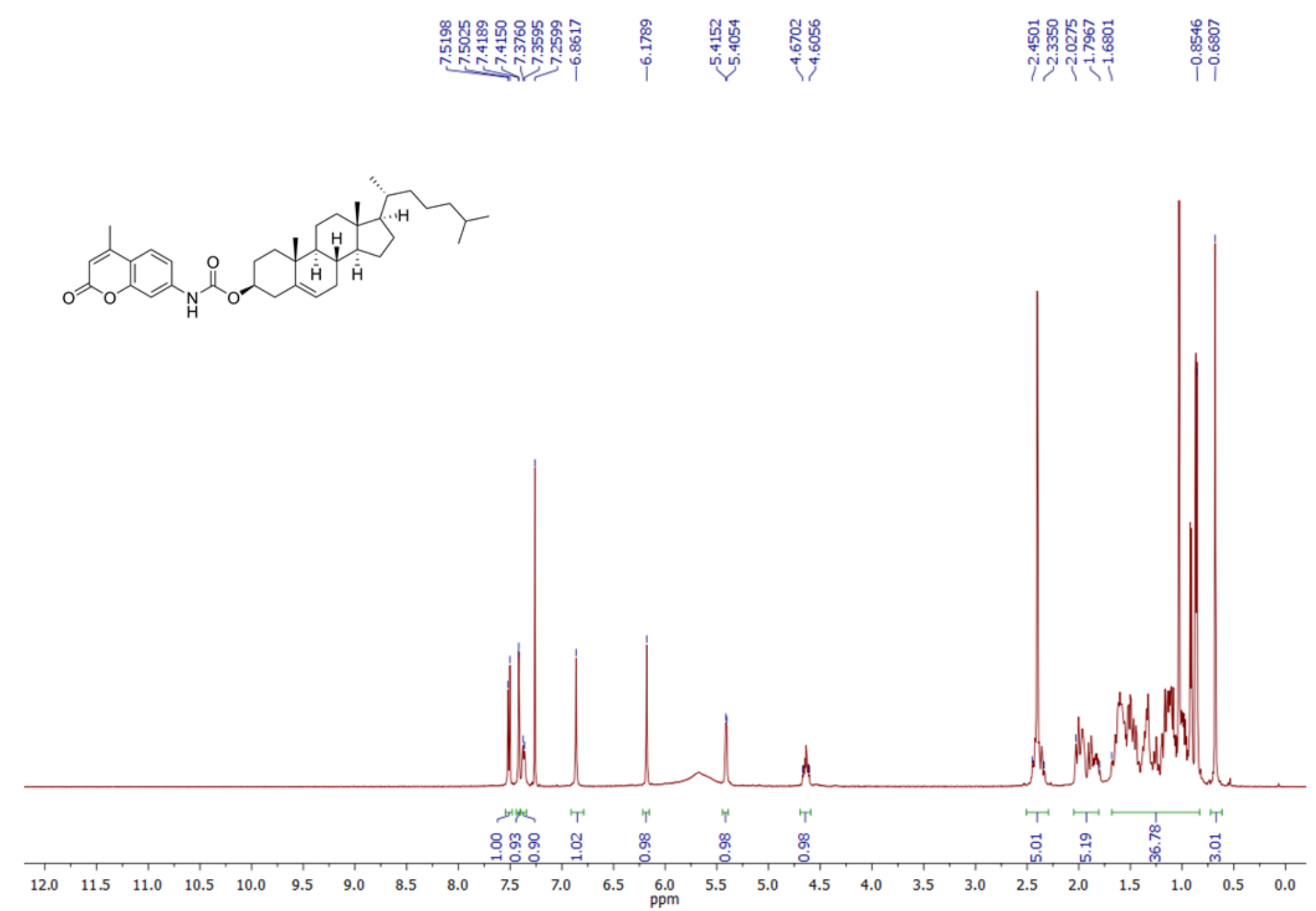

Figure S99: ${ }^{1} \mathrm{H}$ NMR $(500 \mathrm{MHz})$ spectrum of 28 in $\mathrm{CDCl}_{3}$. 


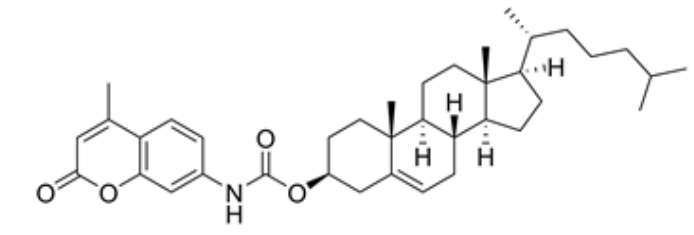

Figure S100: ${ }^{13} \mathrm{C}$ NMR (125 MHz) spectrum of 28 in $\mathrm{CDCl}_{3}$. 

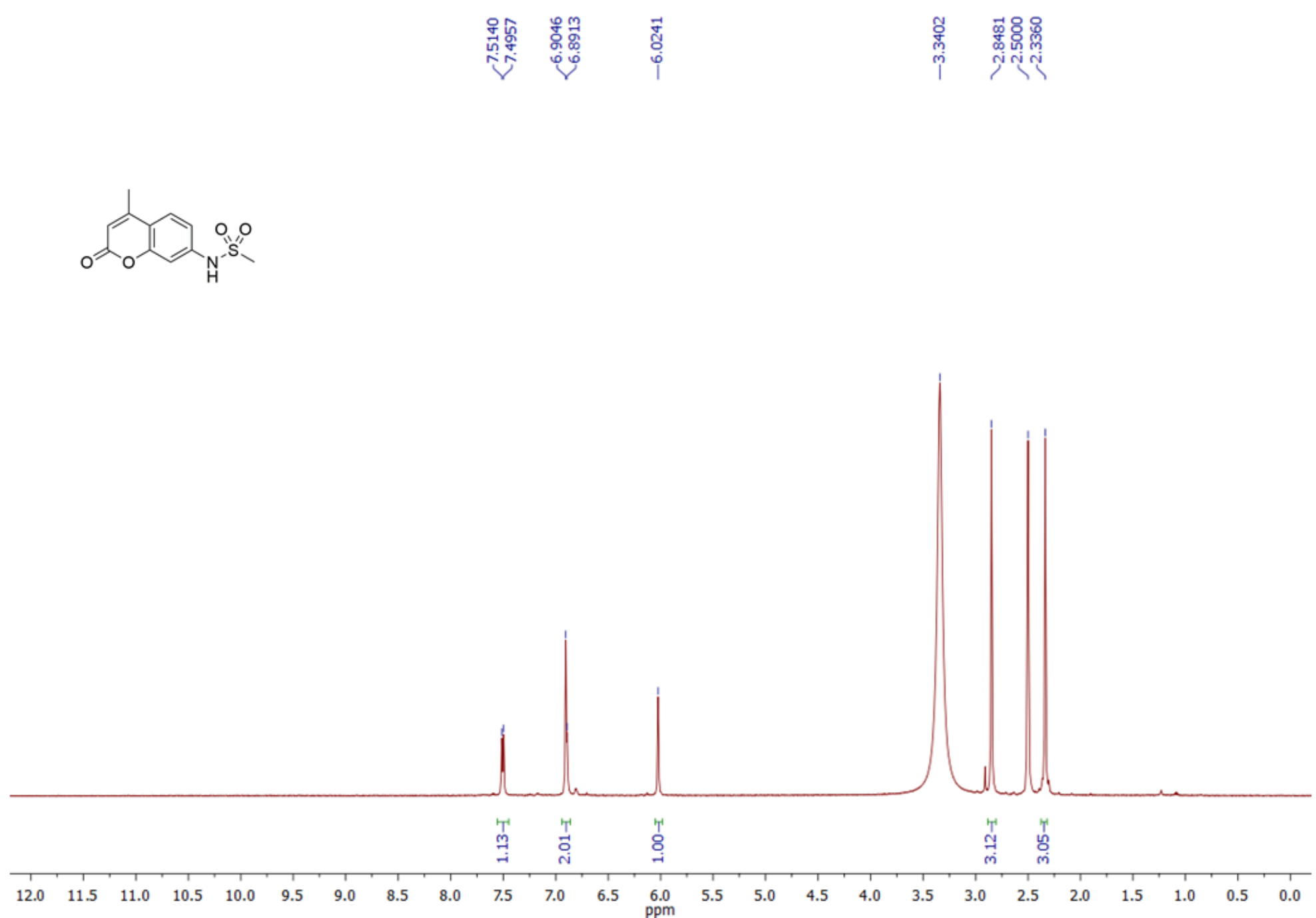

Figure S101: ${ }^{1} \mathrm{H}$ NMR $(500 \mathrm{MHz})$ spectrum of 29 in DMSO- $d_{6}$ 


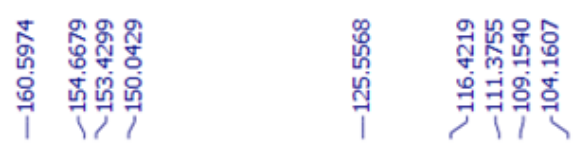

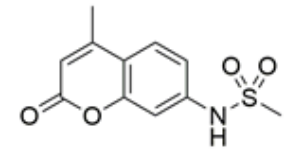
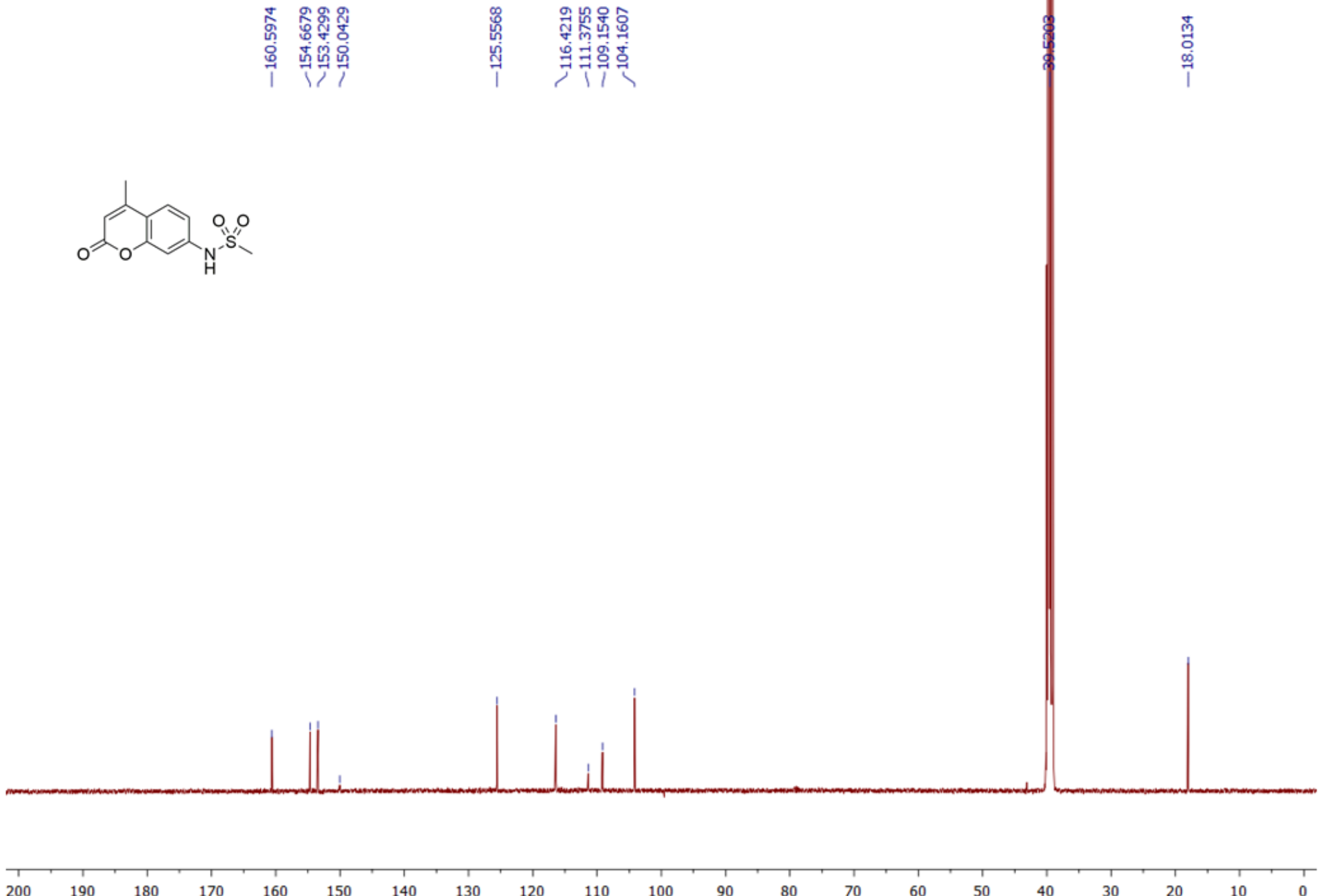

100

80

60

50

40

Figure S102: ${ }^{13} \mathrm{C}$ NMR $(125 \mathrm{MHz})$ spectrum of 29 in DMSO- $d_{6}$. 
+TOF MS: Exp 1, 0.4027 to 0.4119 min from Sample 1(SMH12-085A) of SMH12-085A.wiff

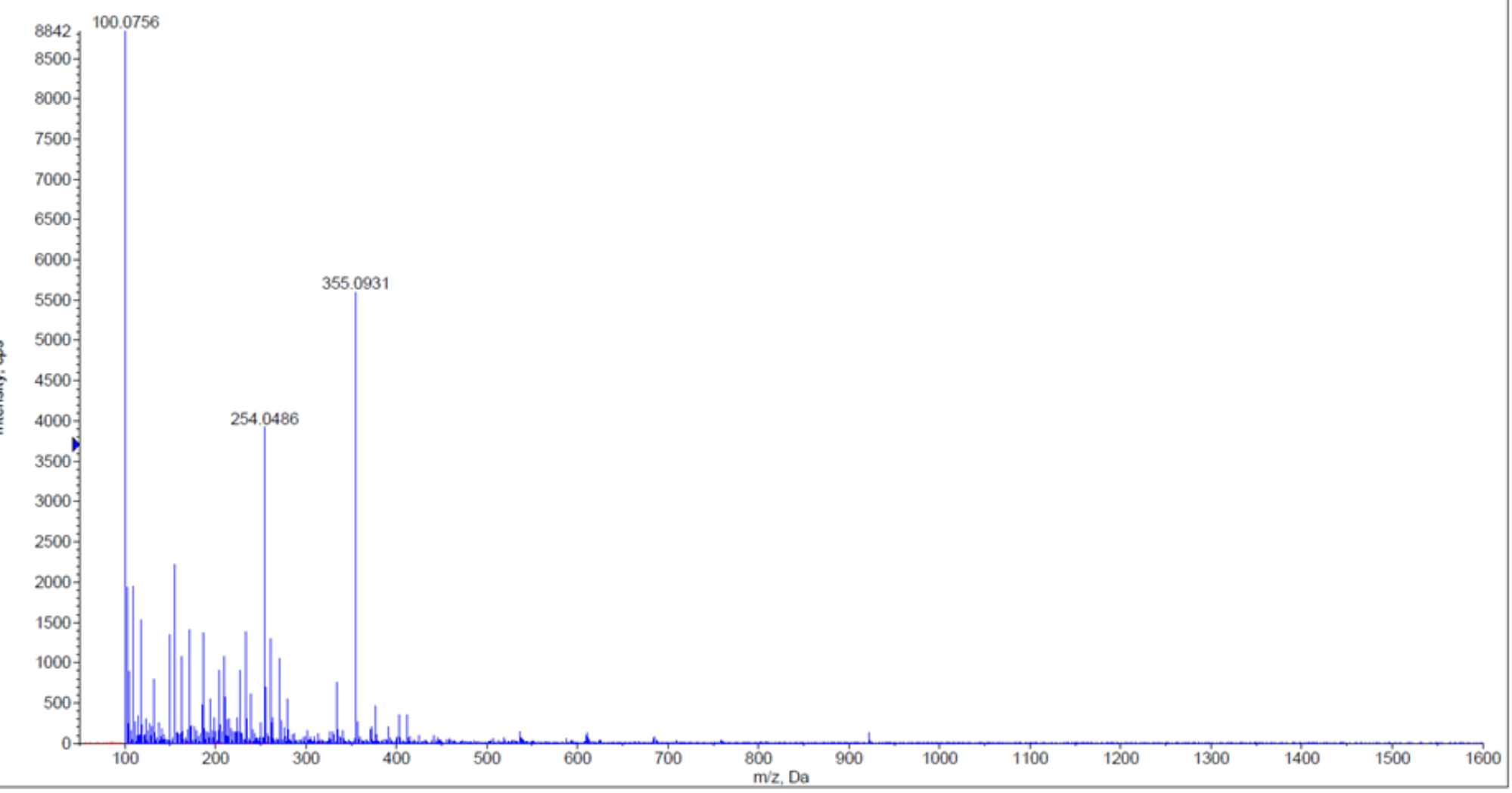

Figure S103: HRMS spectrum of 29. 
Chromatogram

SMH12-085A SMH12-085A.lcd

$\mathrm{mAU}$

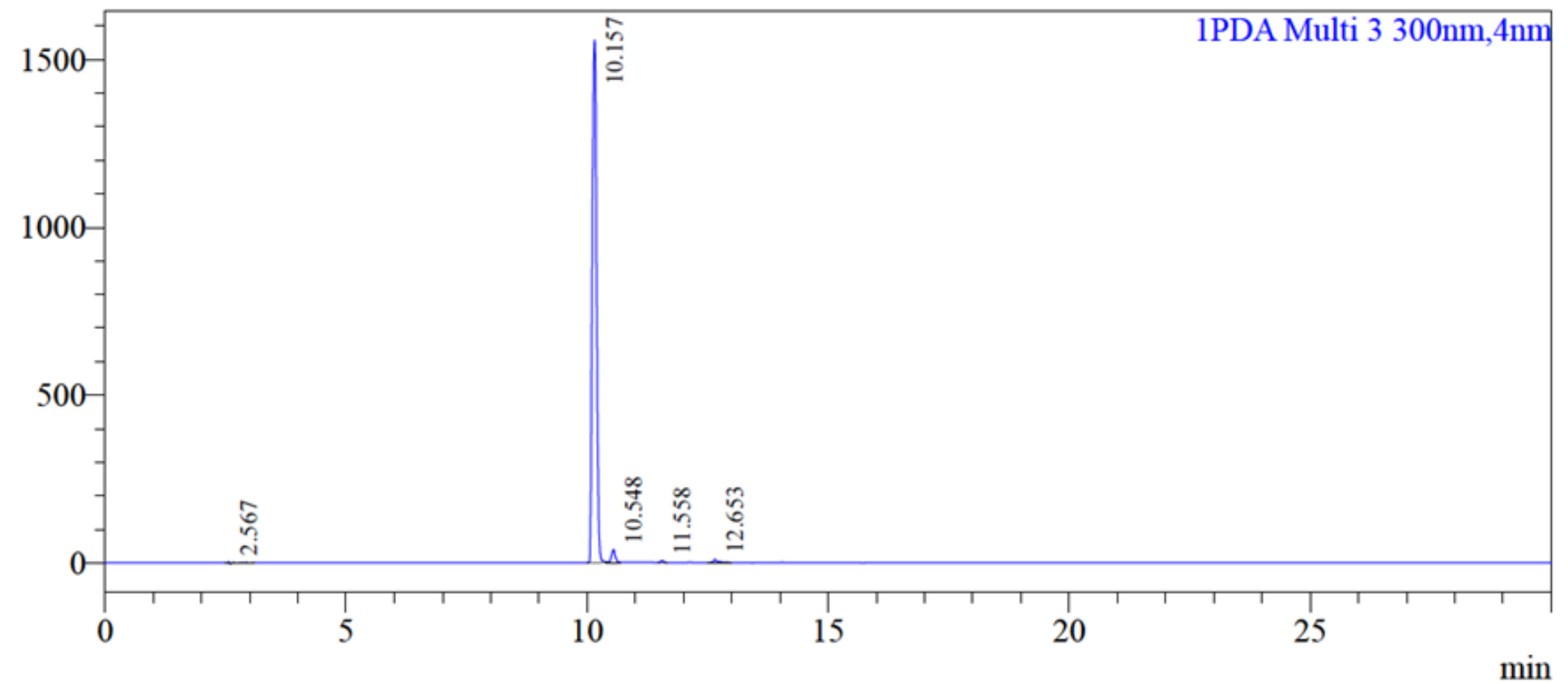

Peak Table

PDA Ch3 300nm
\begin{tabular}{|r|r|r|r|r|l|}
\hline \multicolumn{1}{|c|}{ Peak $\#$} & Ret. Time & \multicolumn{1}{c|}{ Area } & Height & \multicolumn{1}{c|}{ Conc. } & \\
\hline 1 & 2.567 & -1672 & 3520 & -0.017 & \\
\hline 2 & 10.157 & 9760576 & 1558261 & 97.113 & \\
\hline 3 & 10.548 & 202440 & 37752 & 2.014 & \\
\hline 4 & 11.558 & 24485 & 6355 & 0.244 & \\
\hline 5 & 12.653 & 64932 & 9904 & 0.646 & \\
\hline Total & & 10050761 & 1615791 & & \\
\hline
\end{tabular}

Figure S104: RP-HPLC trace of 29. 


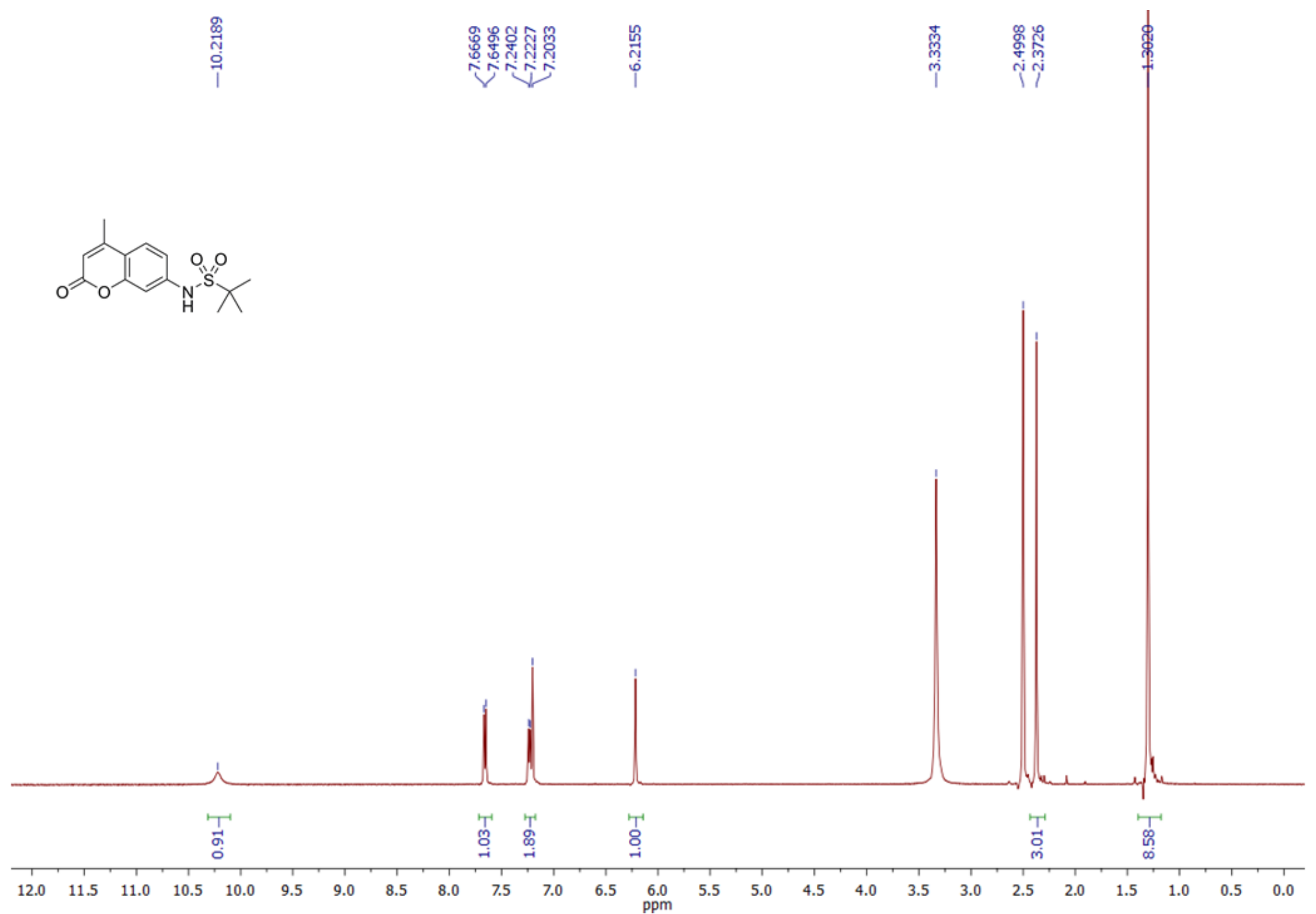

Figure S105: ${ }^{1} \mathrm{H}$ NMR $(500 \mathrm{MHz})$ spectrum of 30 in DMSO- $d_{6}$. 

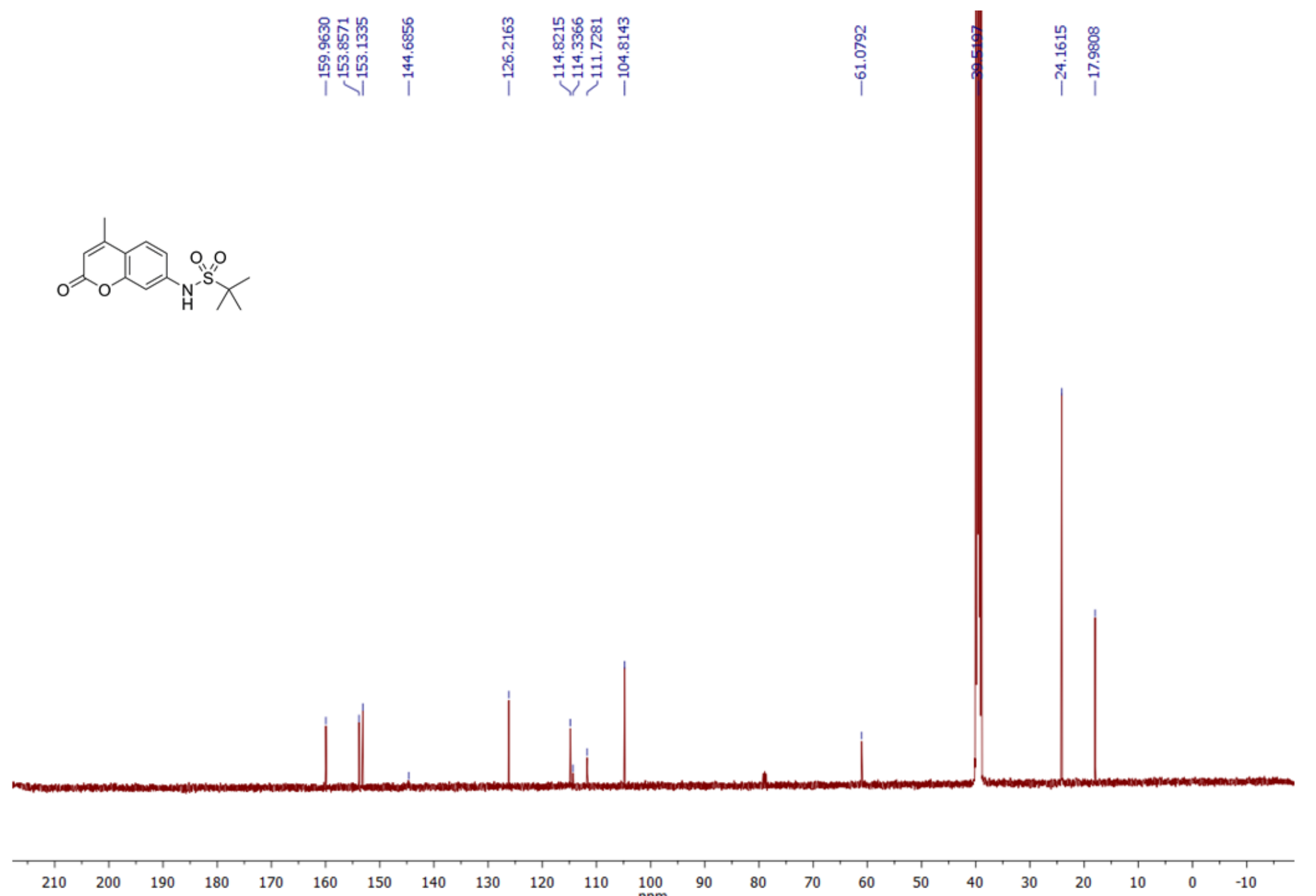

Figure S106: ${ }^{13} \mathrm{C}$ NMR (125 MHz) spectrum of 30 in DMSO- $d_{6}$ 
+TOF MS: Exp 1, 0.1154 to 0.1339 min from Sample 1 (SMHH12-087A) of SMH12-087A.wiff
$a=7.02672166248776460 \theta-004,10=8.60491553706196450 \theta-001$ (DuoSpray ())

$\operatorname{Max} 2.4 \mathrm{e} 5 \mathrm{cps}$

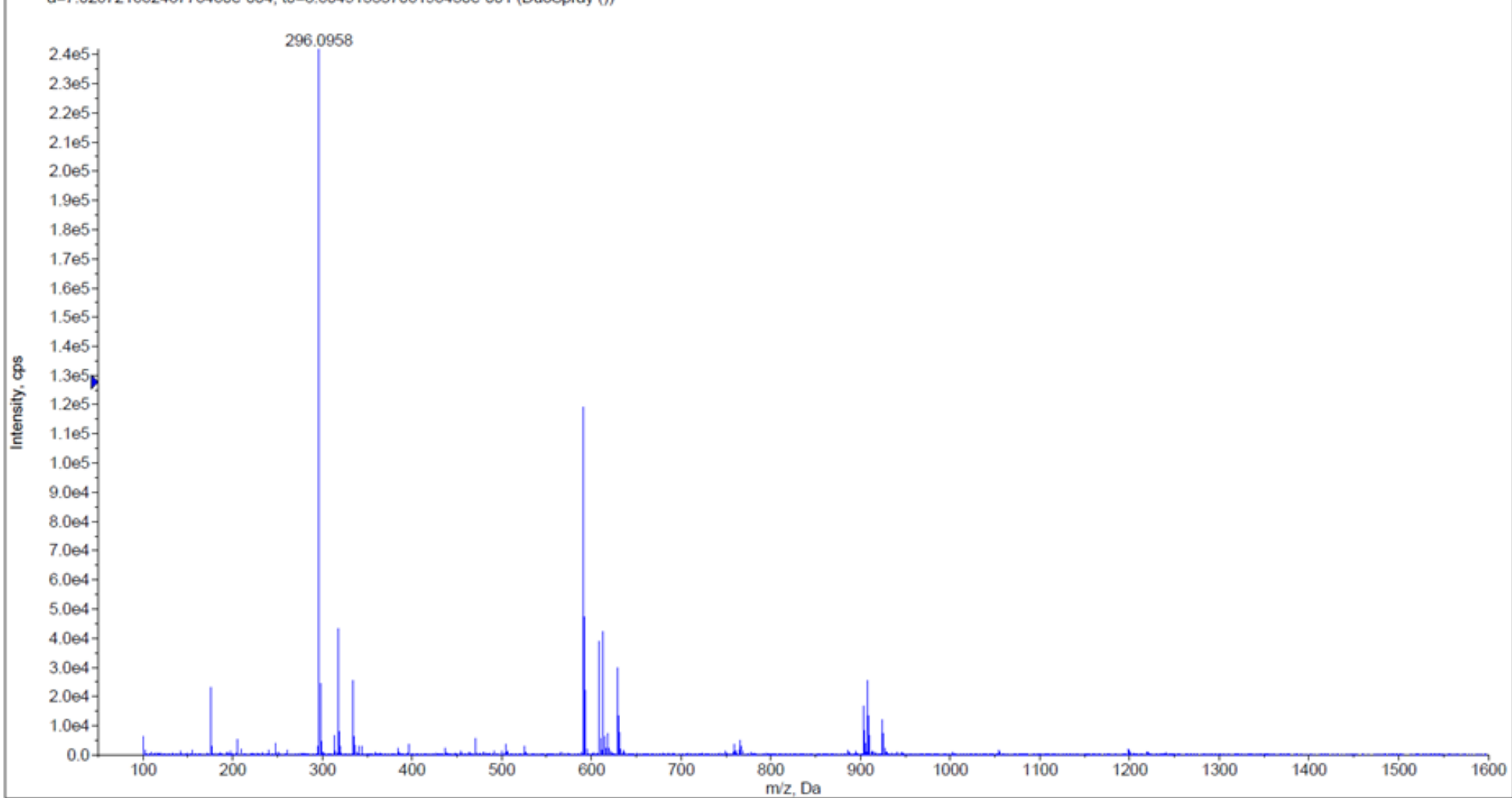

Figure S107: HRMS spectrum of $\mathbf{3 0}$. 
Chromatogram

mAU

SMH12-087A SMH12-087A.led

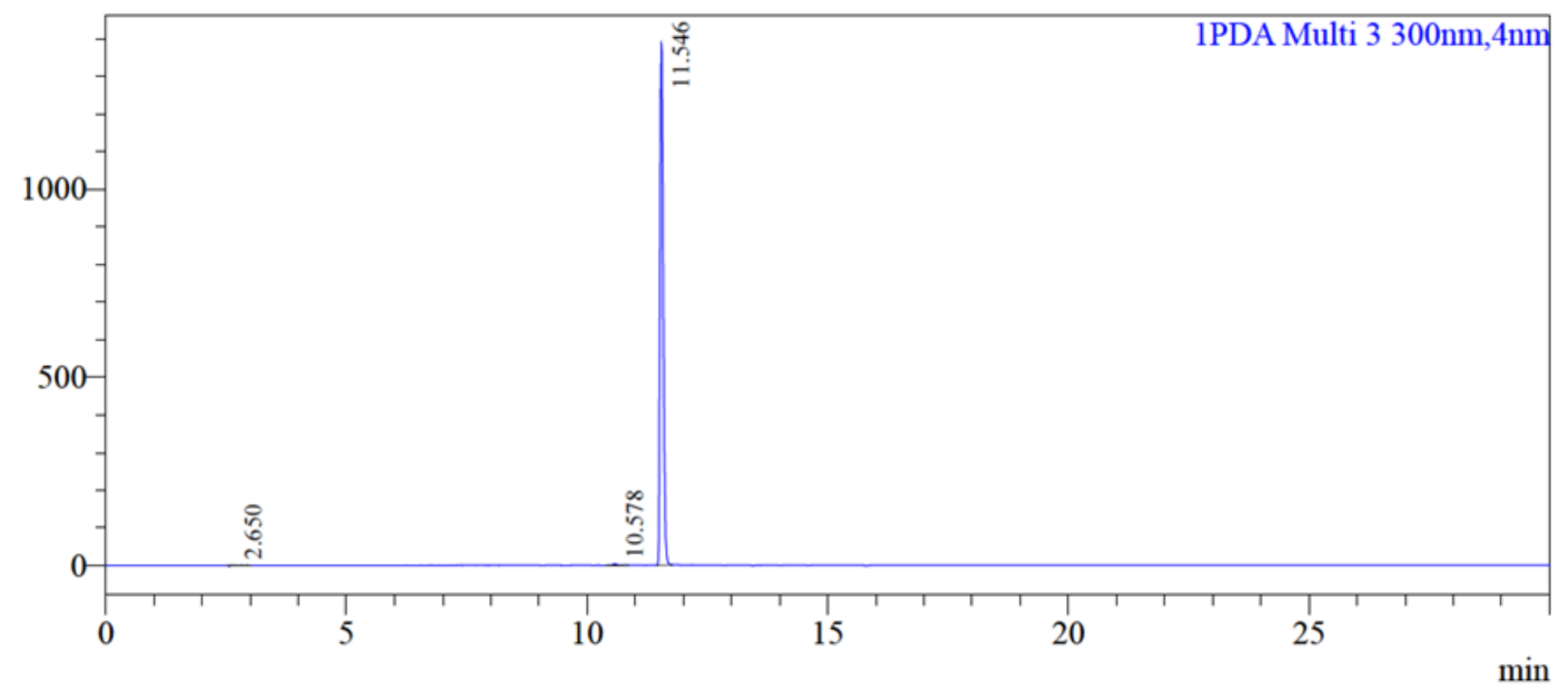

Peak Table

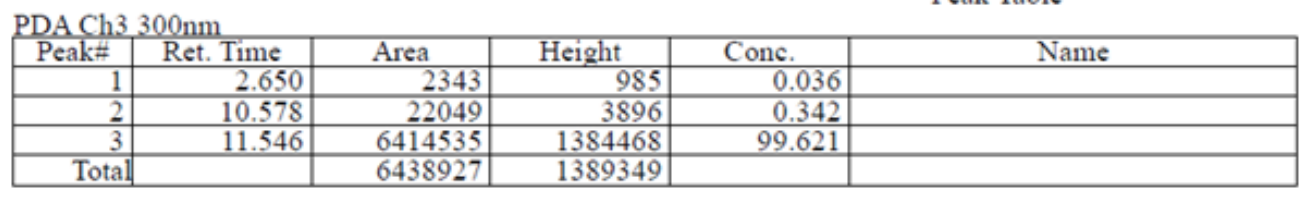

Figure S108: RP-HPLC trace of $\mathbf{3 0 .}$ 


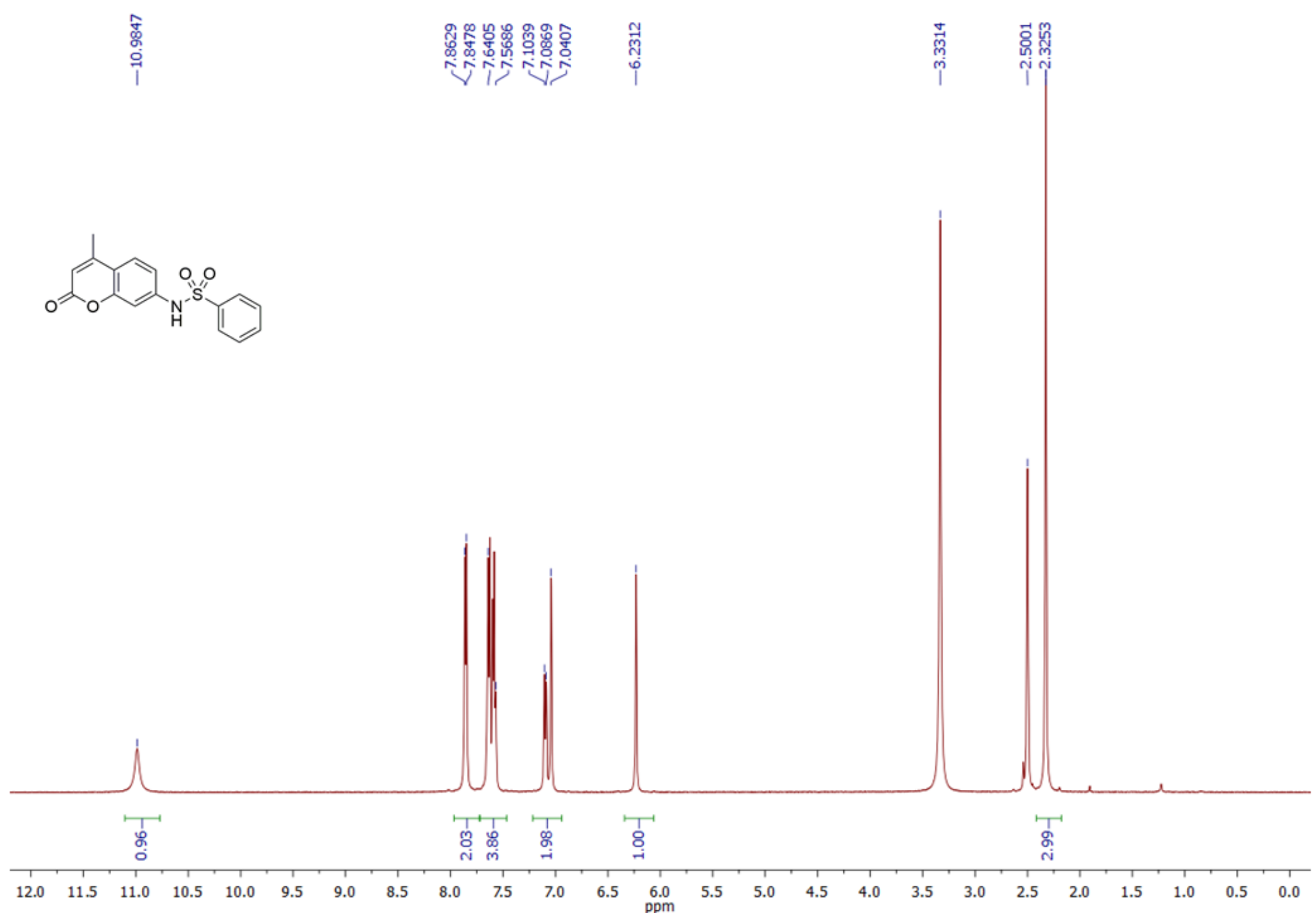

Figure S109: ${ }^{1} \mathrm{H}$ NMR (500 MHz) spectrum of 31 in DMSO- $d_{6}$ 


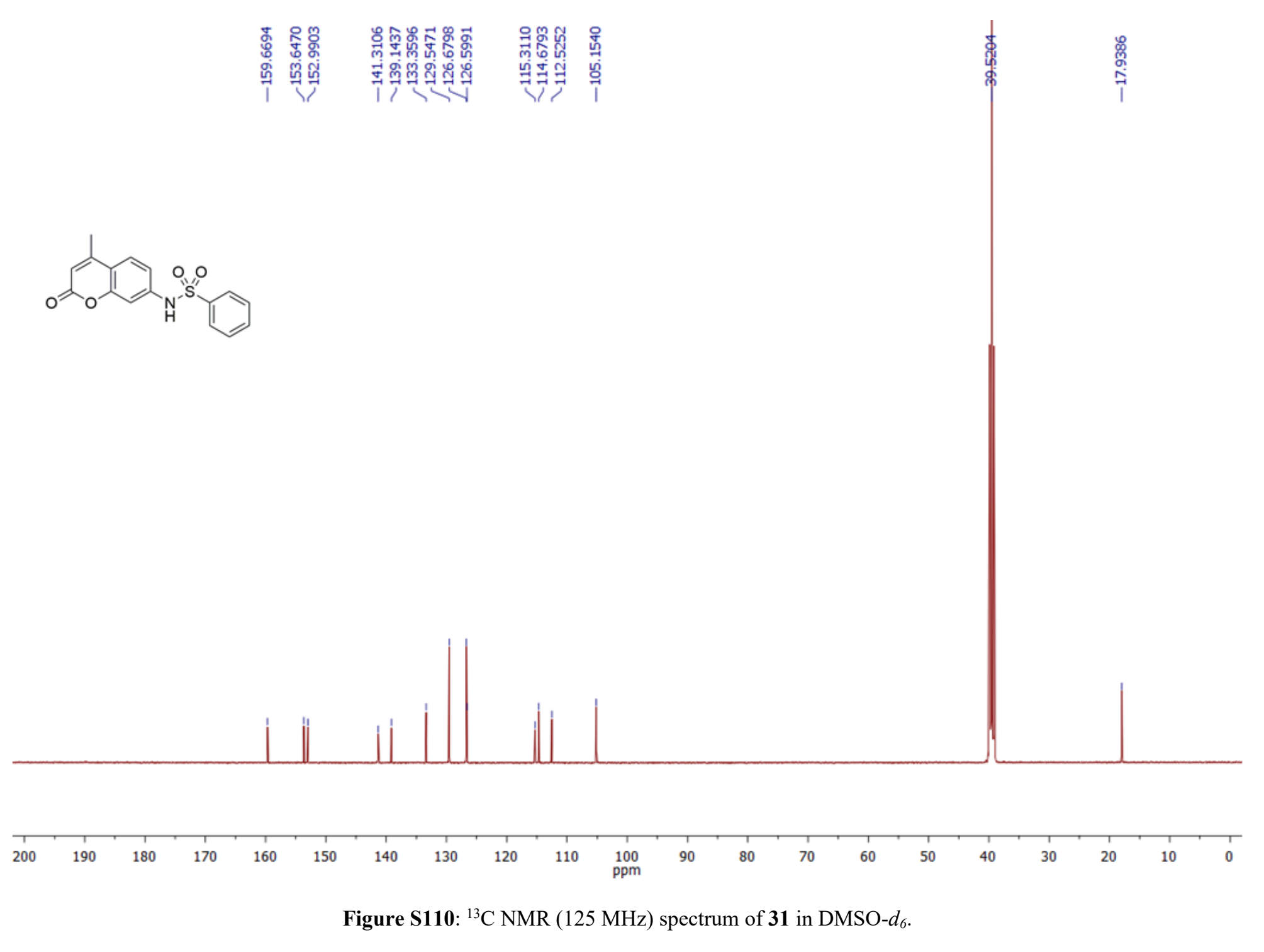


+TOF MS: Exp 1, 0.3470 to 0.3563 min from Sample 1(SMH12-103B) of SMH12-1038. wiff

Max. $9.504 \mathrm{cps}$. (Duospray ())

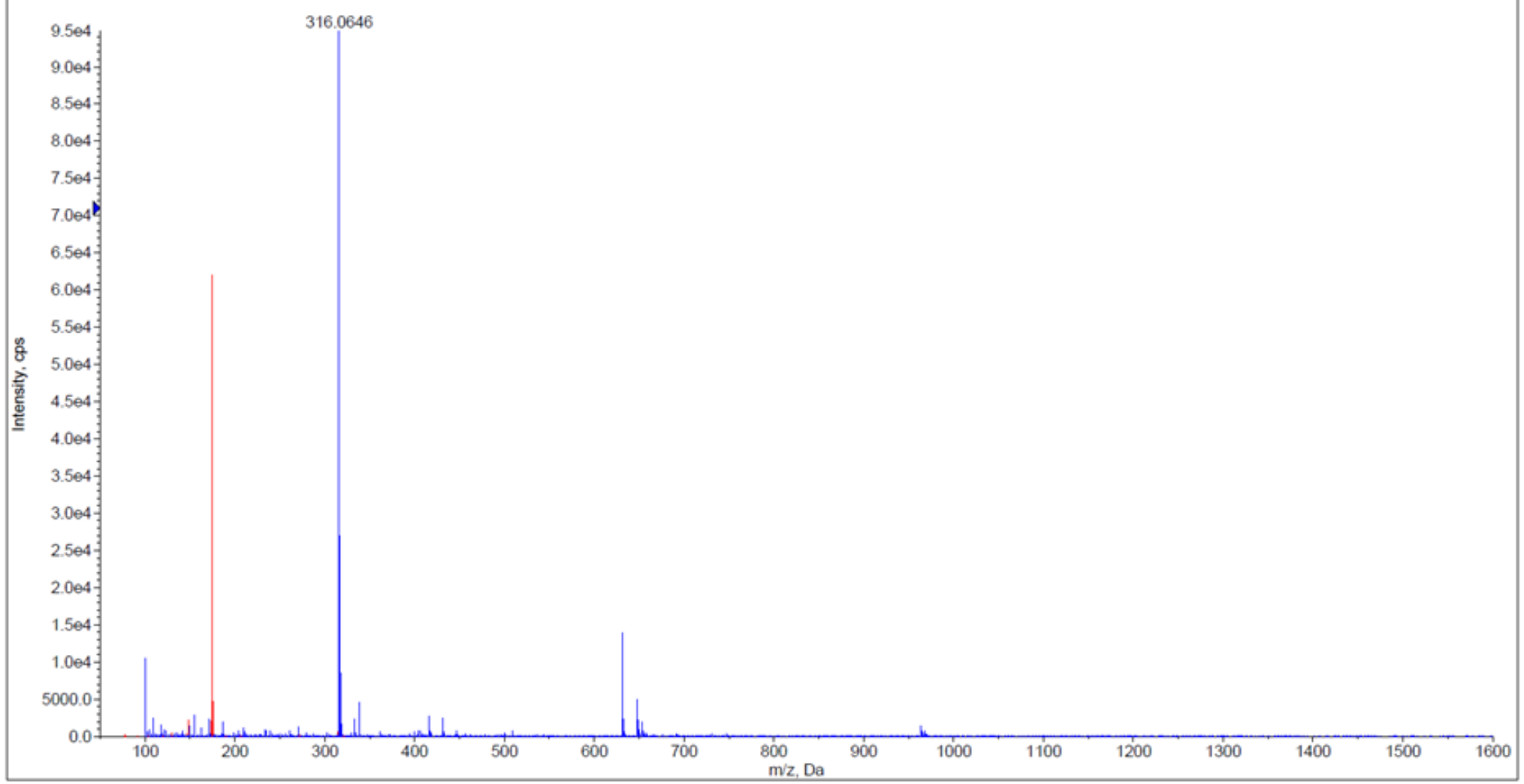

Figure S111: HRMS spectrum of 31. 
Chromatogram

SMH12-103B SMH12-103B.lcd

mAU

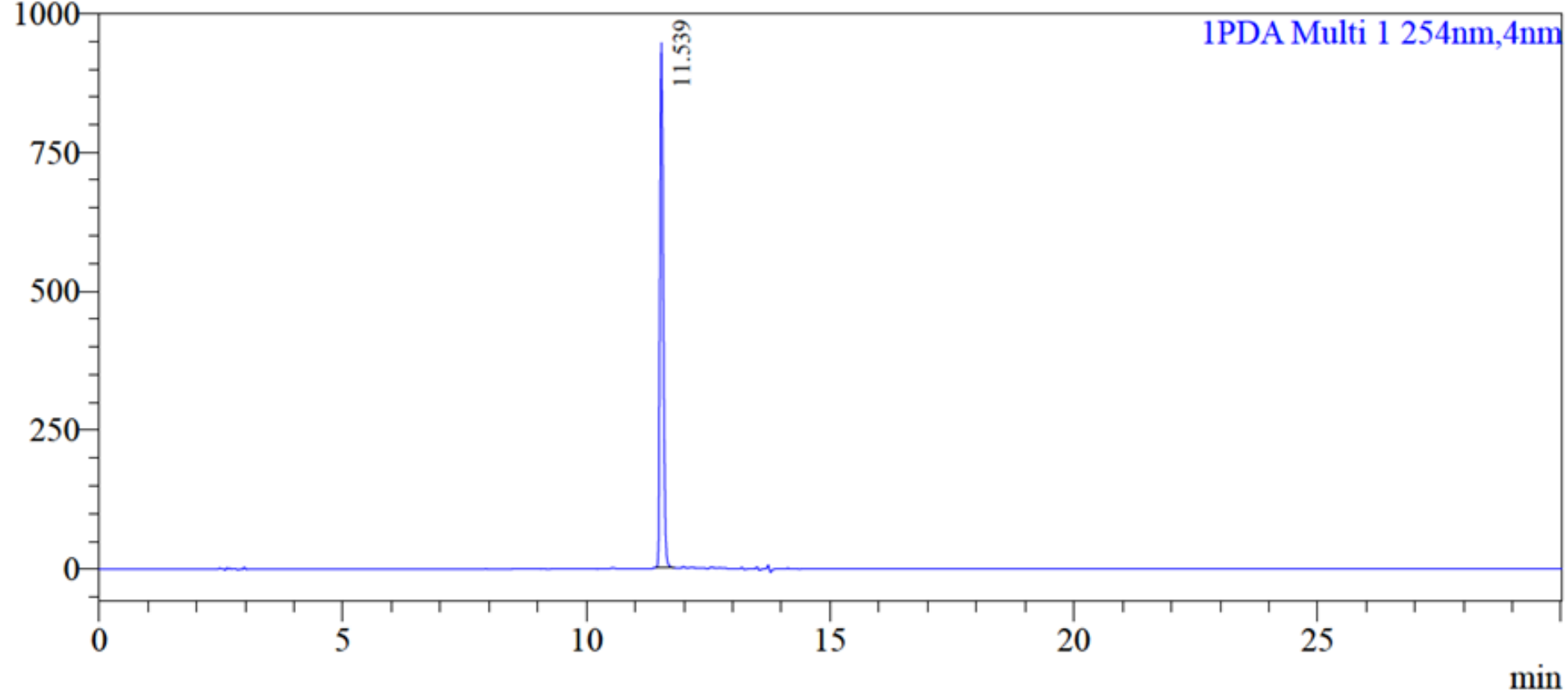

Peak Table

PDA Ch1 $254 \mathrm{~nm}$
\begin{tabular}{|r|r|r|r|r|r|}
\hline Peak\# & Ret. Time & \multicolumn{1}{|c|}{ Area } & \multicolumn{1}{c|}{ Height } & Conc. & Name \\
\hline 1 & 11.539 & 4423211 & 945689 & 100.000 & \\
\hline Total & & 4423211 & 945689 & & \\
\hline
\end{tabular}

Figure S112: RP-HPLC trace of 31. 


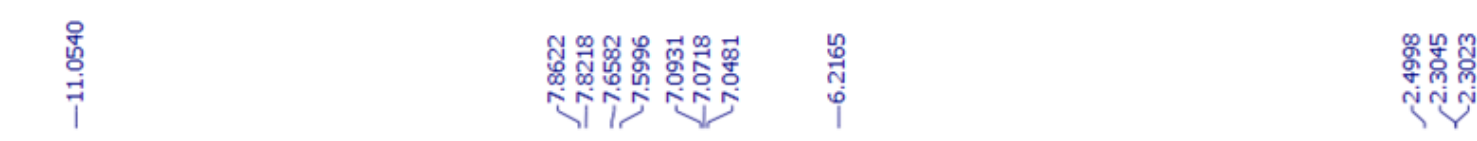
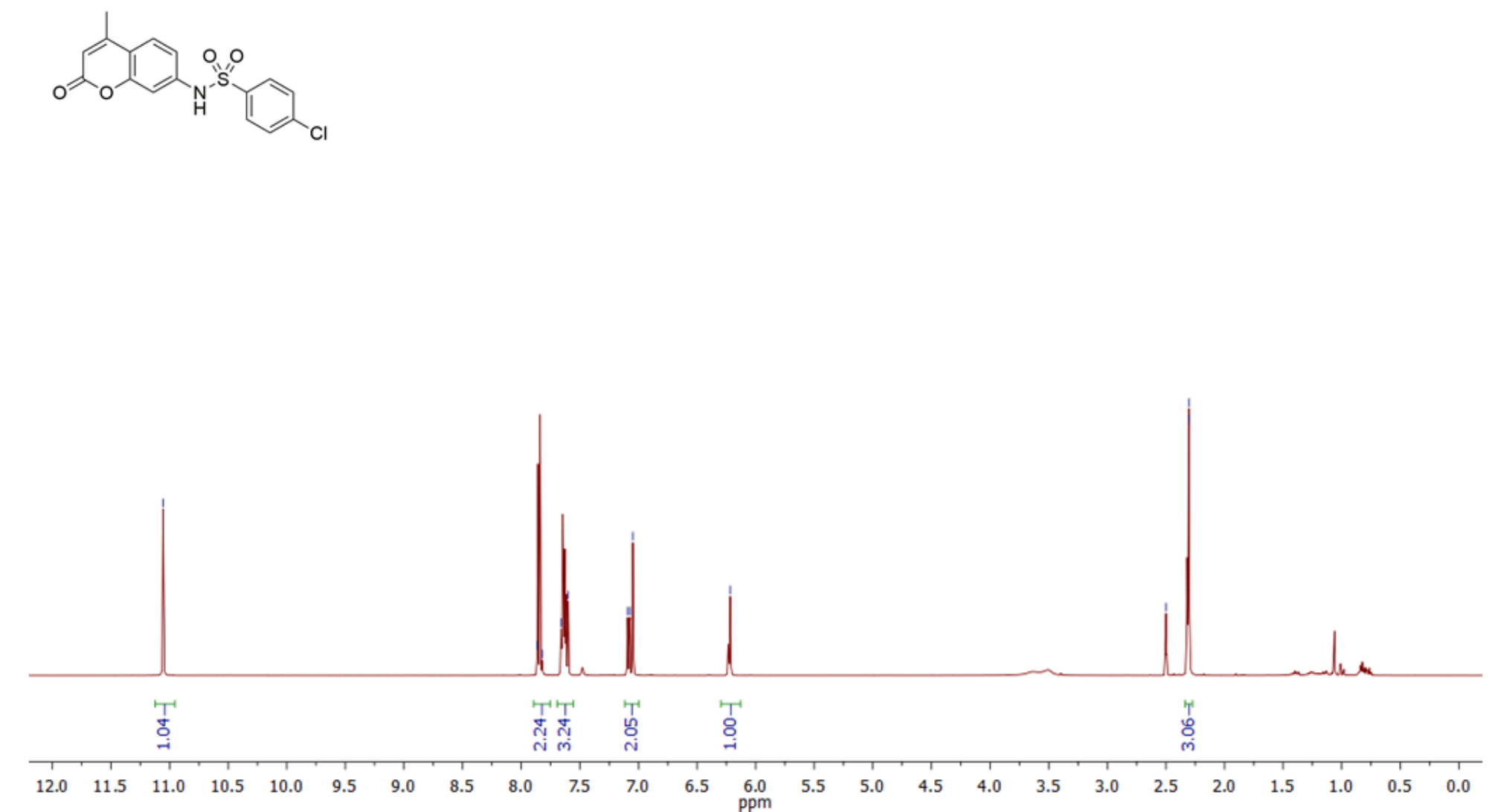

Figure S113: ${ }^{1} \mathrm{H}$ NMR (500 MHz) spectrum of 32 in DMSO- $d_{6}$. 

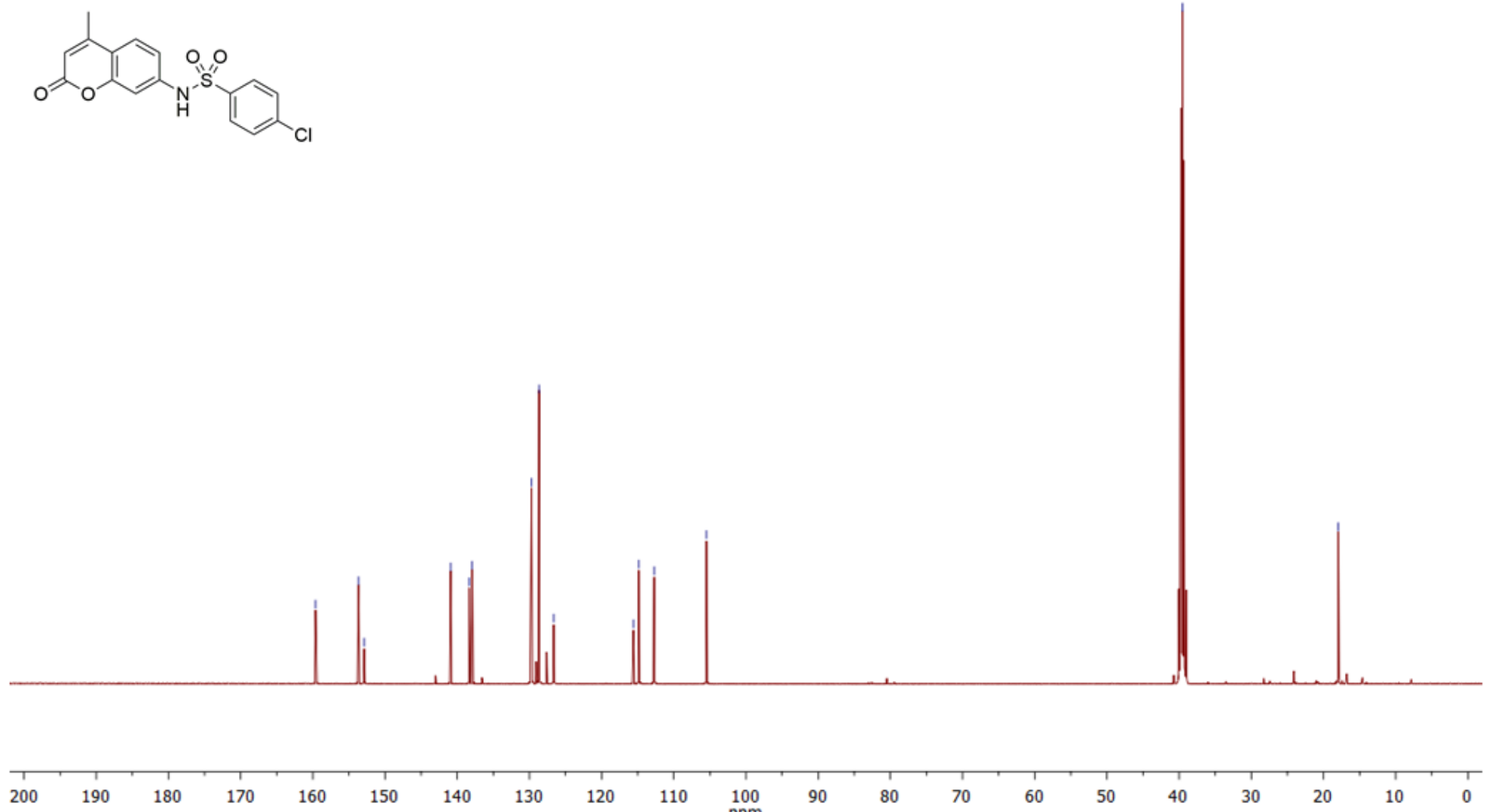

Figure S114: ${ }^{13} \mathrm{C}$ NMR (125 MHz) spectrum of 32 in DMSO- $d_{6}$ 
- +TOF MS: Exp 1, 0.3934 to 0.4120 min from Sample 1 (SMH10-1018) of SMH 10-1018 wiff different calibrabons (DuoSpray ())

Max $5.704 \mathrm{cps}$

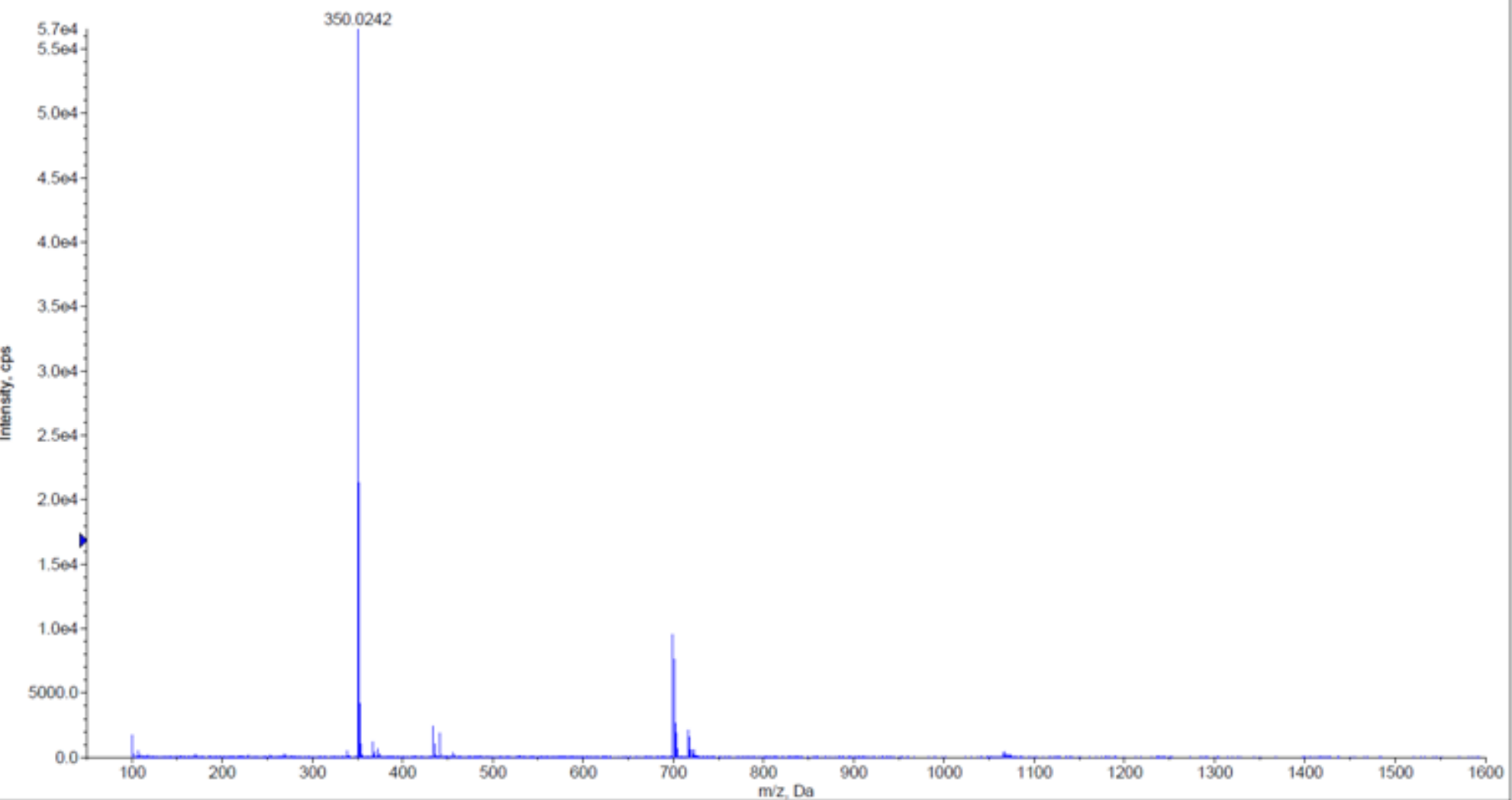

Figure S115: HRMS spectrum of 32. 
Chromatogram

mAU

SMH10-101B SMH10-101B.led

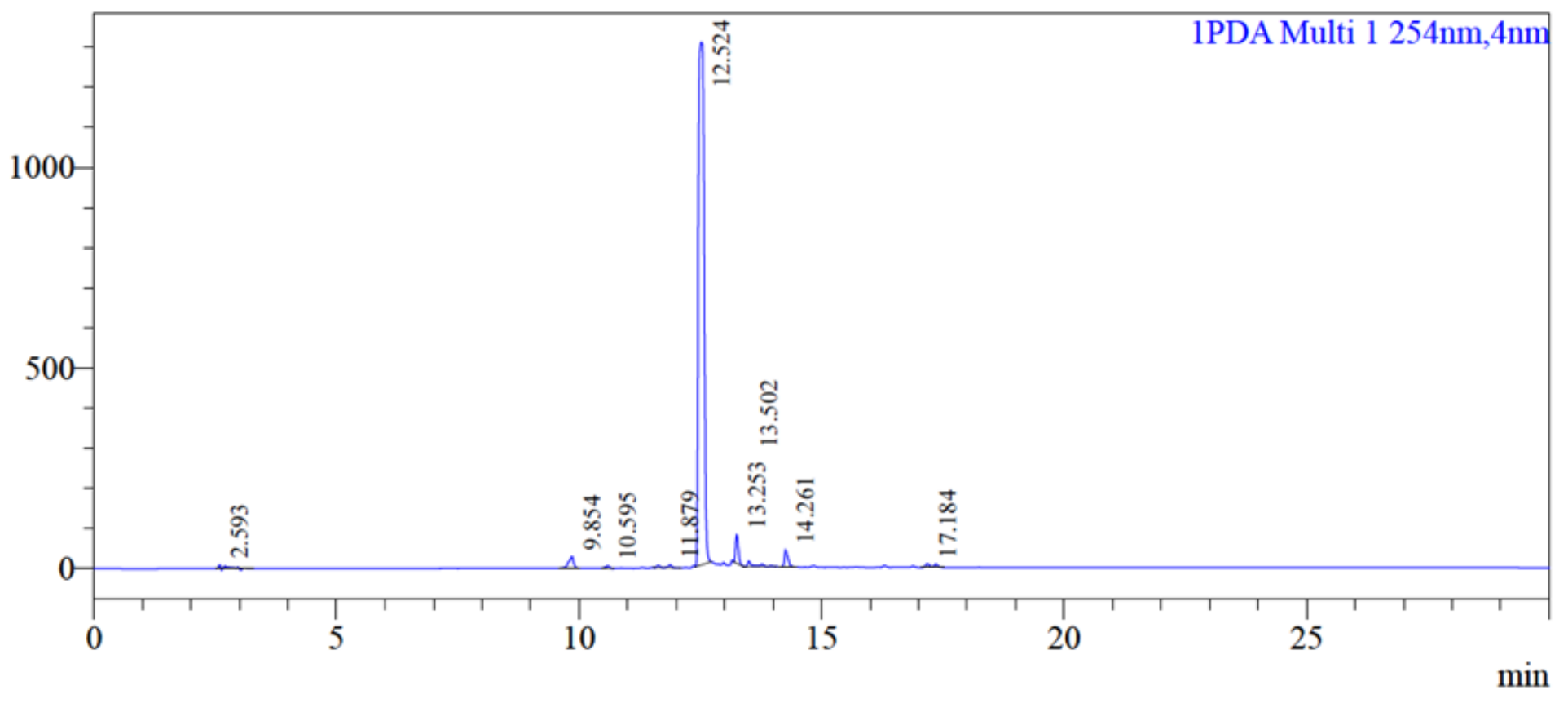

Peak Table

PDA Ch1 $254 \mathrm{~nm}$
\begin{tabular}{|r|r|r|r|r|l|}
\hline Peak= & Ret. Time & \multicolumn{1}{c|}{ Area } & \multicolumn{1}{c|}{ Height } & Conc. & \\
\hline 1 & 2.593 & 61172 & 8789 & 0.531 & \\
\hline 2 & 9.854 & 208182 & 29661 & 1.806 & \\
\hline 3 & 10.595 & 34298 & 6009 & 0.298 & \\
\hline 4 & 11.879 & 54416 & 7028 & 0.472 & \\
\hline 5 & 12.524 & 10480644 & 1302296 & 90.920 & \\
\hline 6 & 13.253 & 278559 & 70612 & 2.417 & \\
\hline 7 & 13.502 & 111459 & 13676 & 0.967 & \\
\hline 8 & 14.261 & 204580 & 41658 & 1.775 & \\
\hline 9 & 17.184 & 94017 & 9259 & 0.816 & \\
\hline Total & & 11527328 & 1488989 & & \\
\hline
\end{tabular}

Figure S116: RP-HPLC trace of 32. 


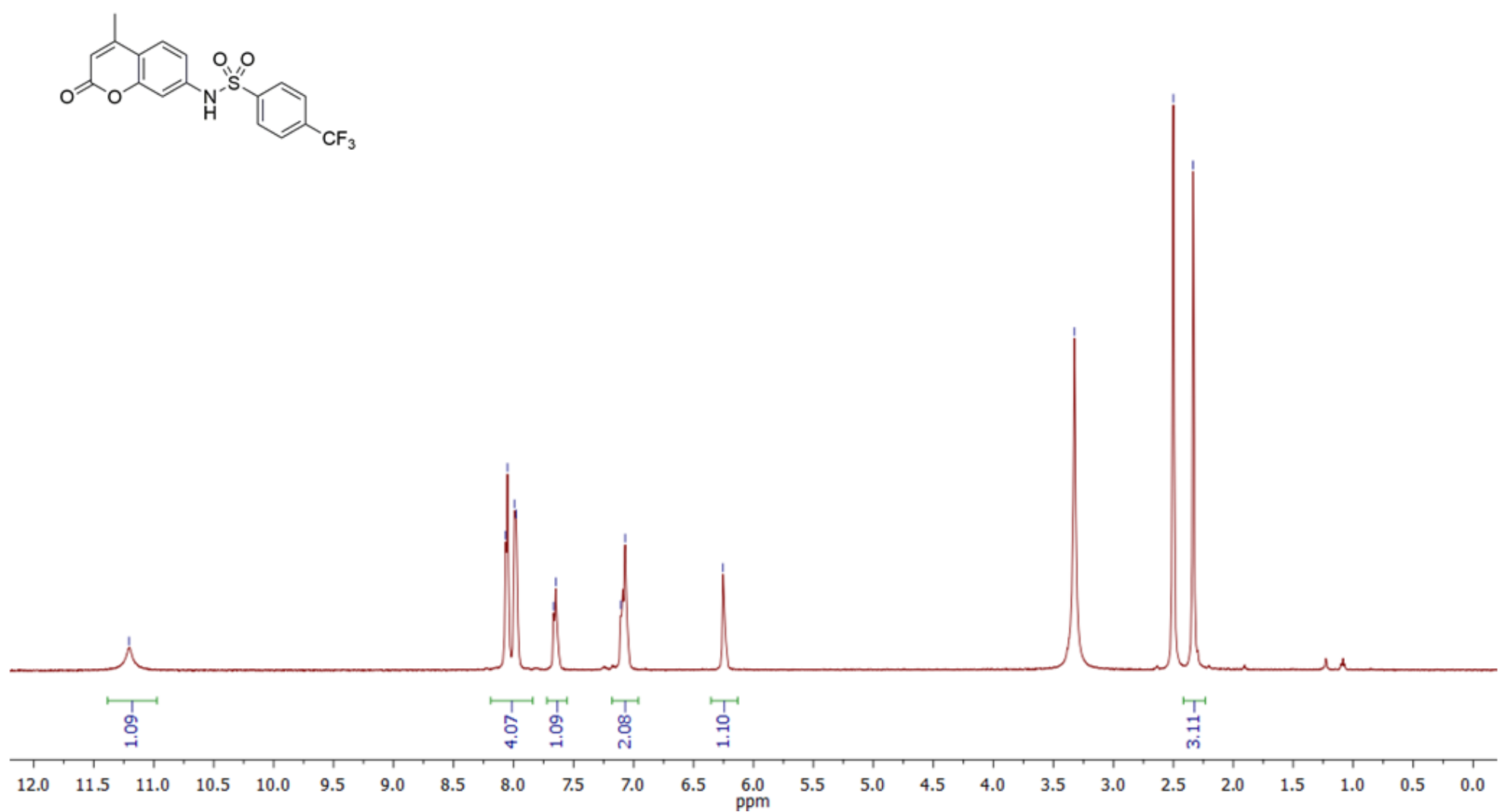

Figure S117: ${ }^{1} \mathrm{H}$ NMR (500 MHz) spectrum of 33 in DMSO- $d_{6}$. 


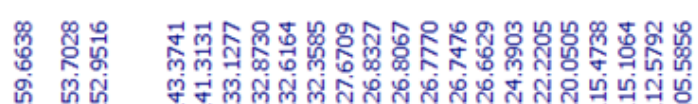

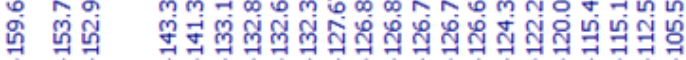

1 र
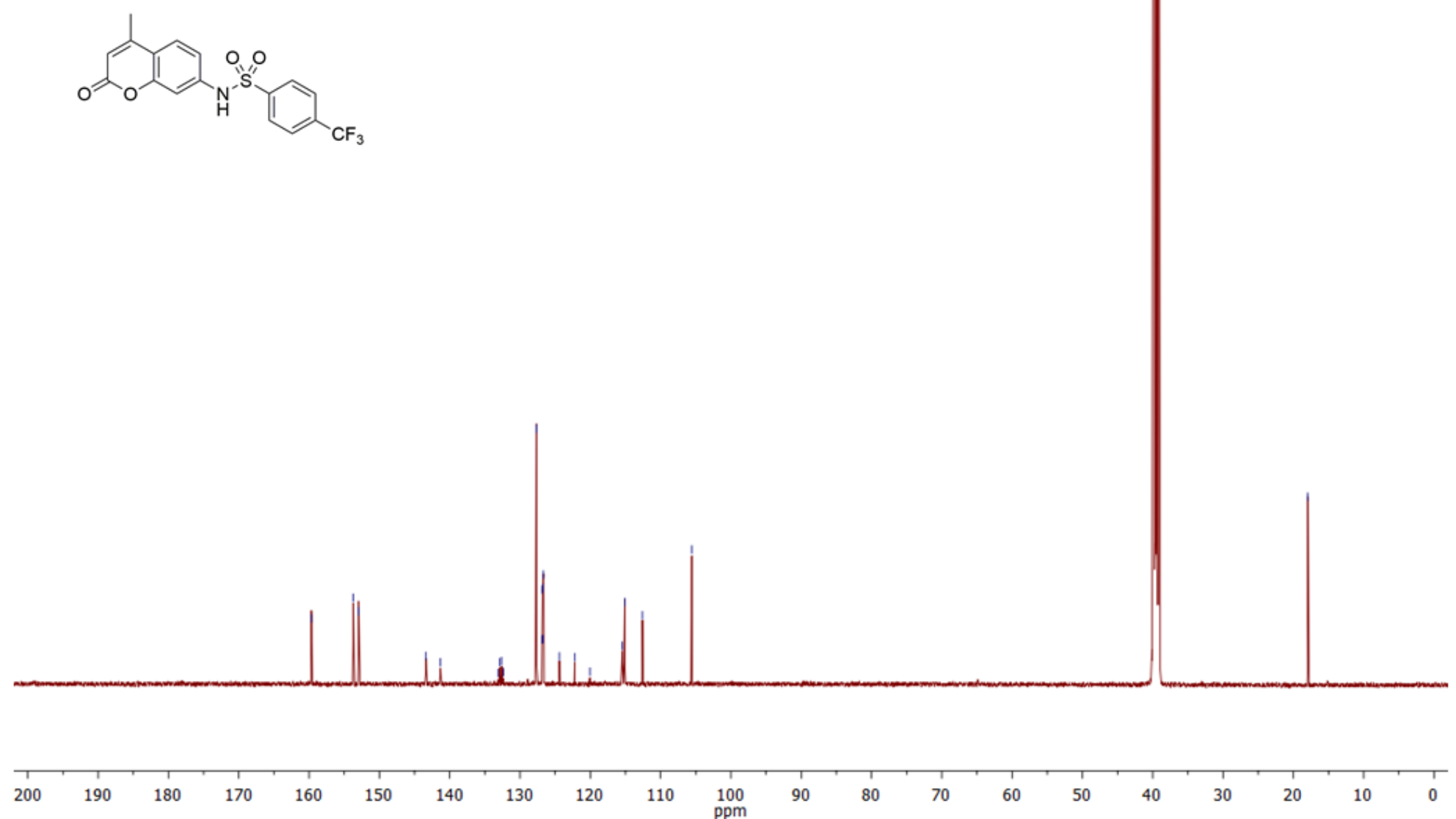

Figure S118: ${ }^{13} \mathrm{C}$ NMR (125 MHz) spectrum of 33 in DMSO- $d_{6}$. 
+TOF MS: Exp 1, 0.1246 to 0.1339 min from Sample 1 (SMH12-105A) of SMH12-105A.wiff

Max. $2.005 \mathrm{cps}$. (DuoSpray ())

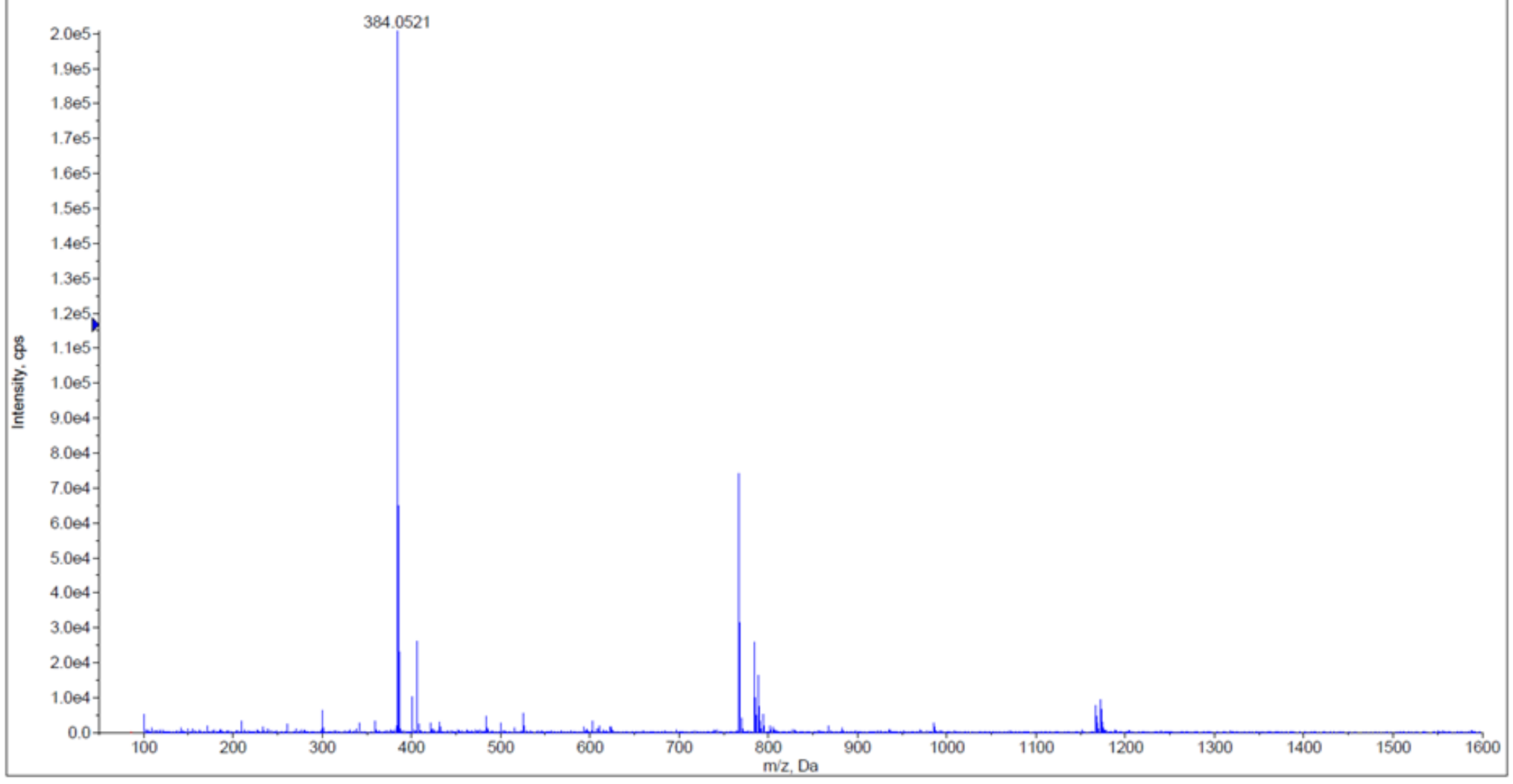

Figure S119: HRMS spectrum of $\mathbf{3 3}$ 
Chromatogram

$\mathrm{mAU}$

SMH12-105A SMH12-105A.lcd

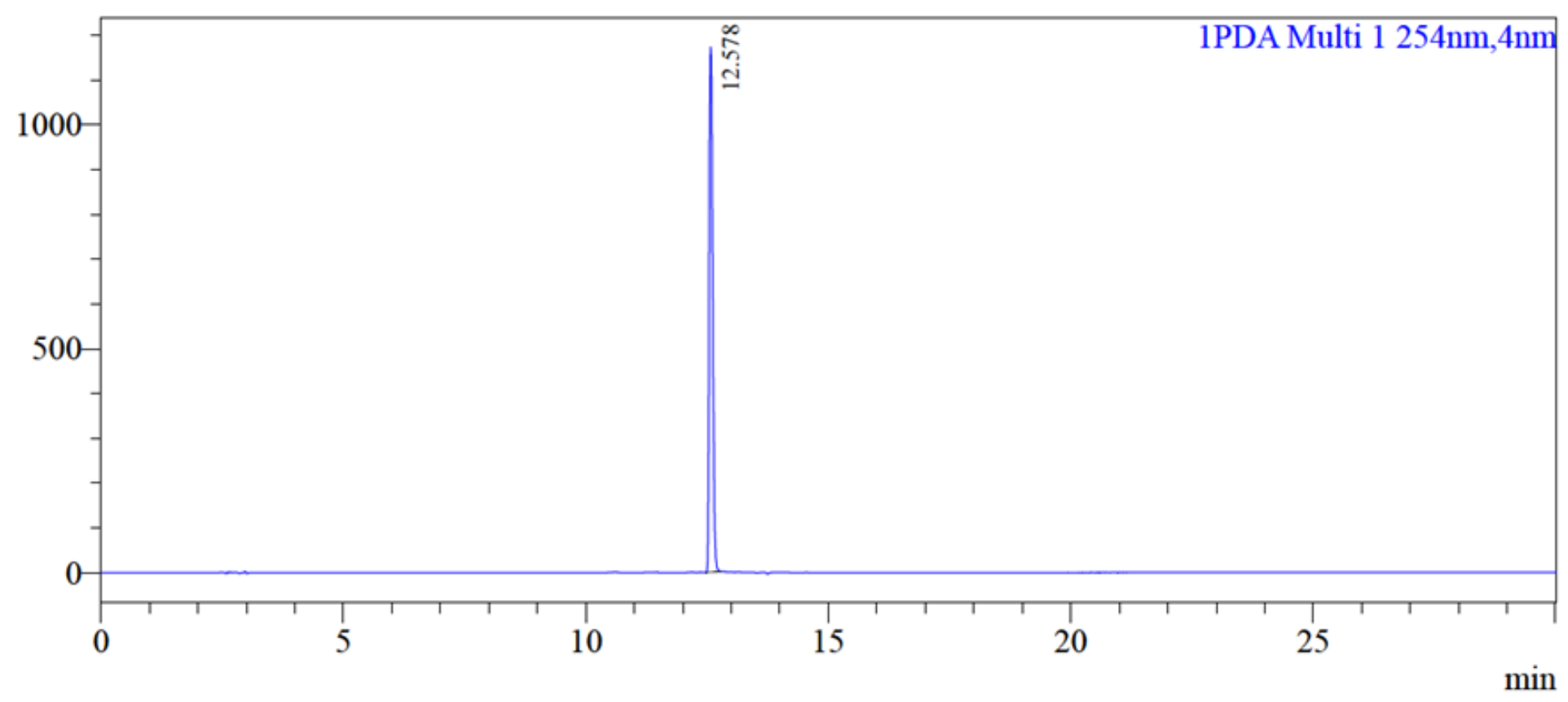

Peak Table

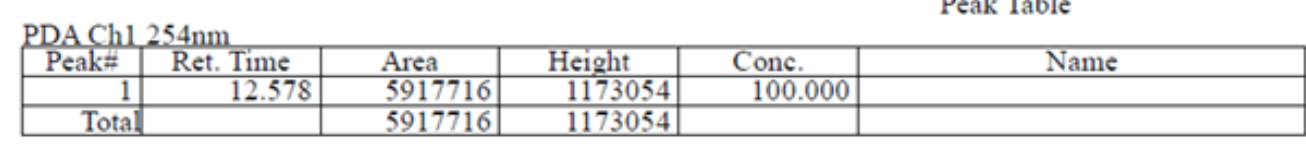

Figure S120: RP-HPLC trace of 33. 


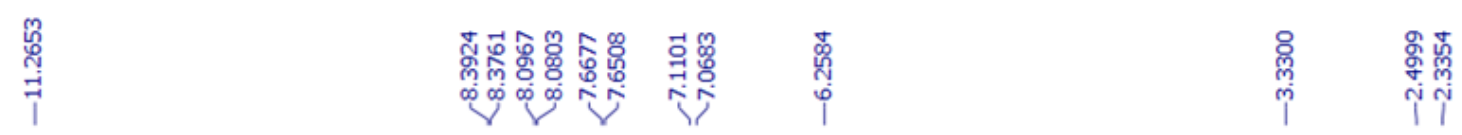

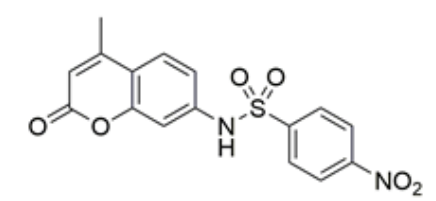

$\mathrm{NO}_{2}$

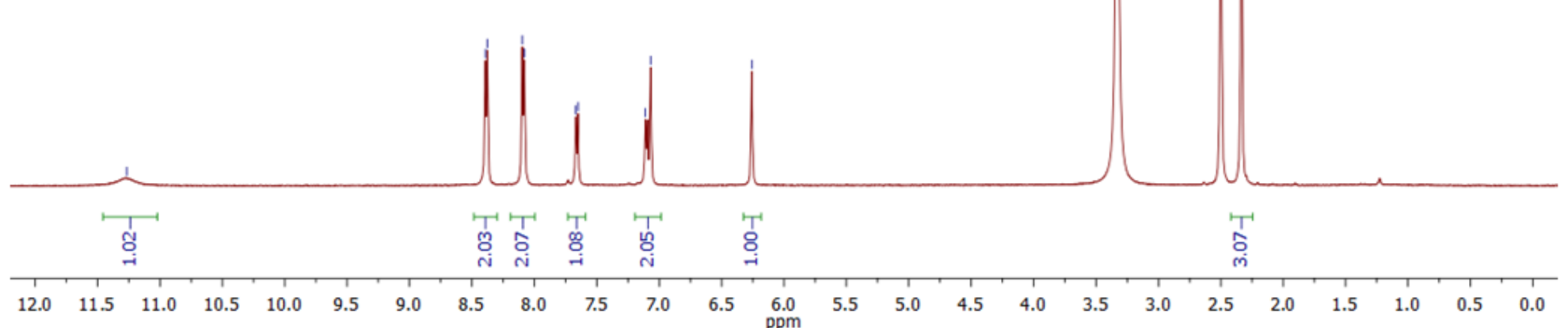

Figure S121: ${ }^{1} \mathrm{H}$ NMR (500 MHz) spectrum of 34 in DMSO- $d_{6}$. 


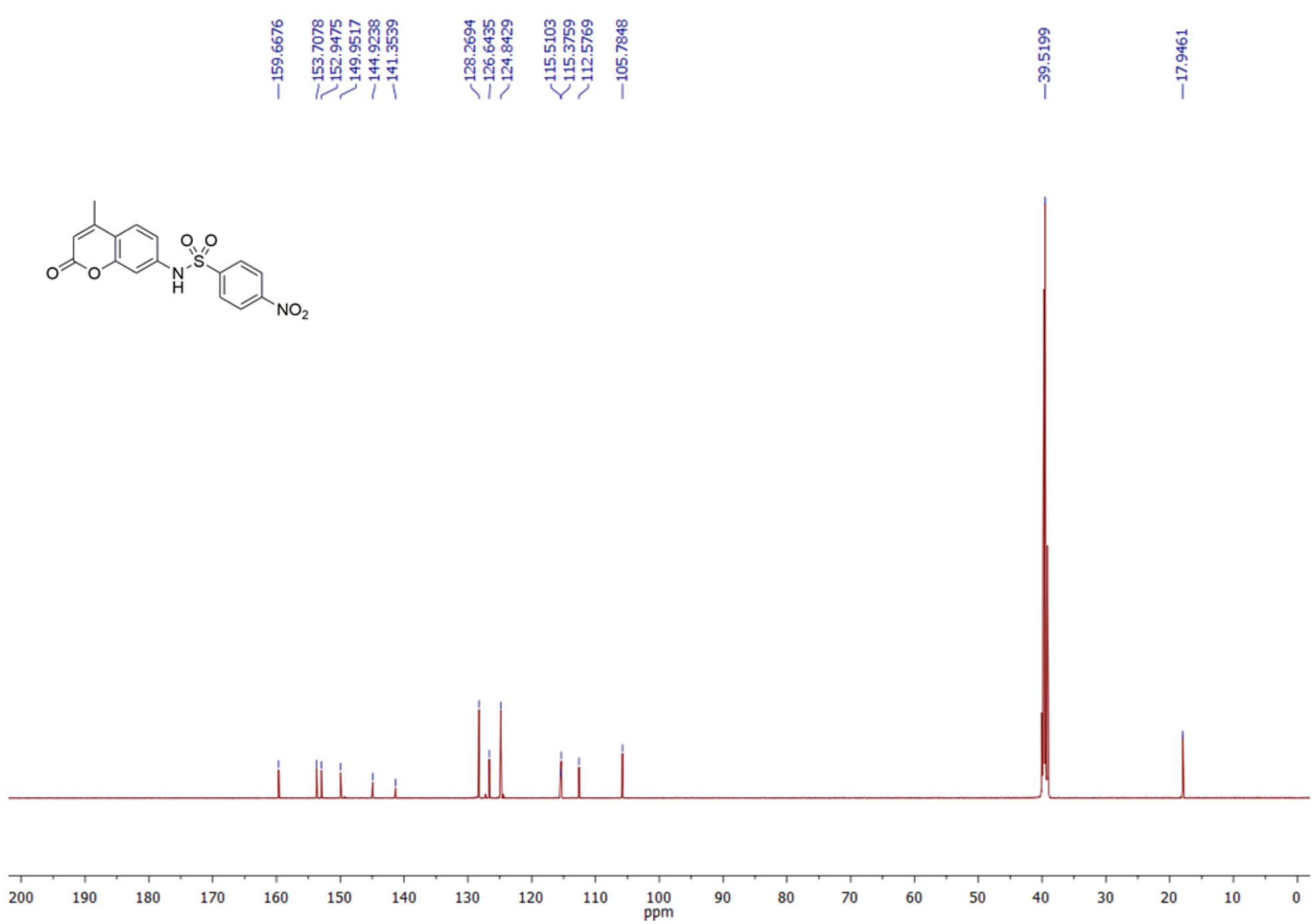

Figure S122: ${ }^{13} \mathrm{C}$ NMR (125 MHz) spectrum of 34 in DMSO- $d_{6}$. 
+TOF MS: Exp 1, 0.2914 min from Sample 1(SMH12-107A) of SMH12-107A.wift

Max. $1.2 e 5 \mathrm{cps}$ $\mathrm{a}=7.02666393403577630 \mathrm{e}-004,10=8.59351478661157690 \mathrm{e}-001$ (DuoSpray ())

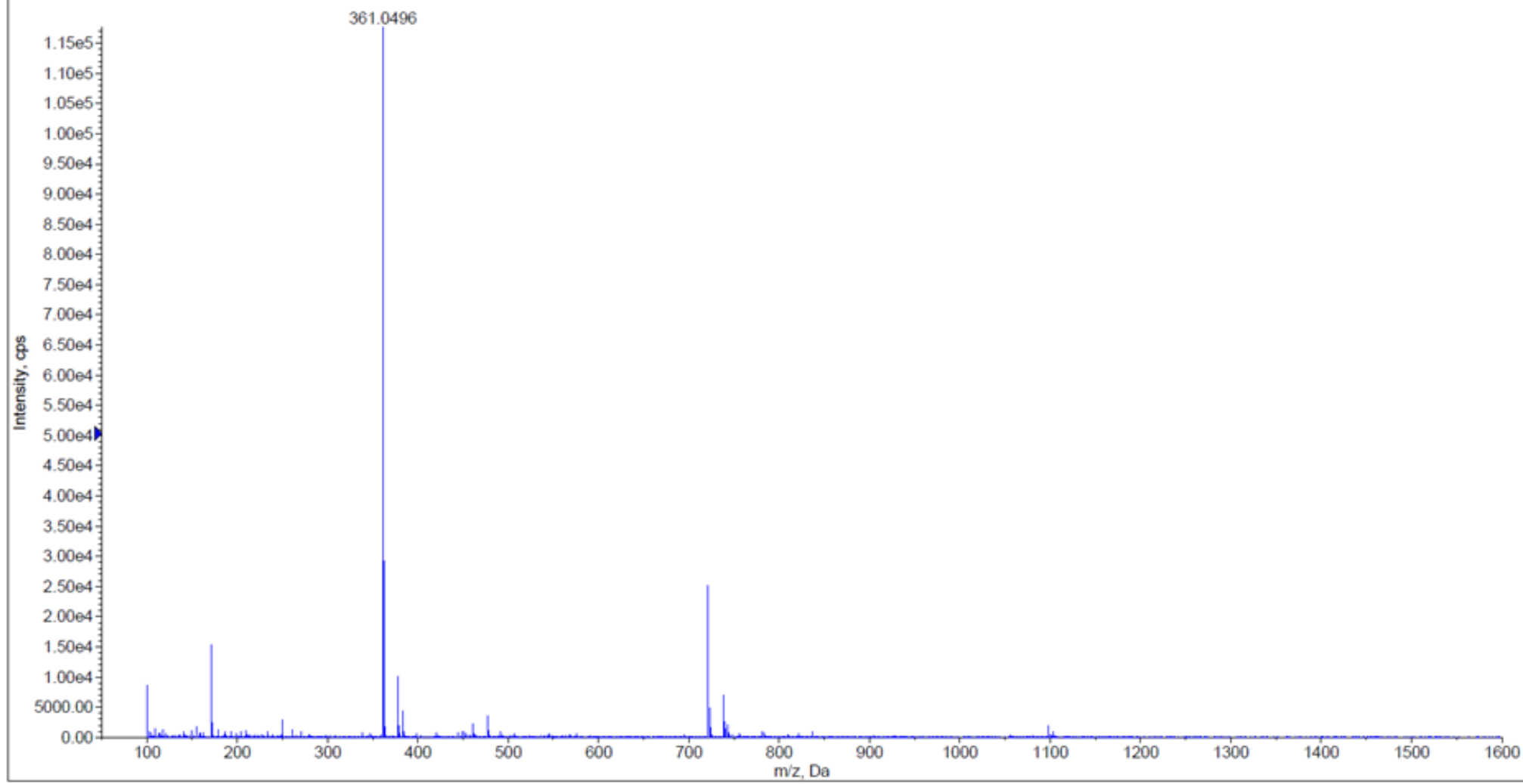

Figure S123: HRMS spectrum of 34. 
Chromatogram

SMH12-107A SMH12-107A.led

mAU

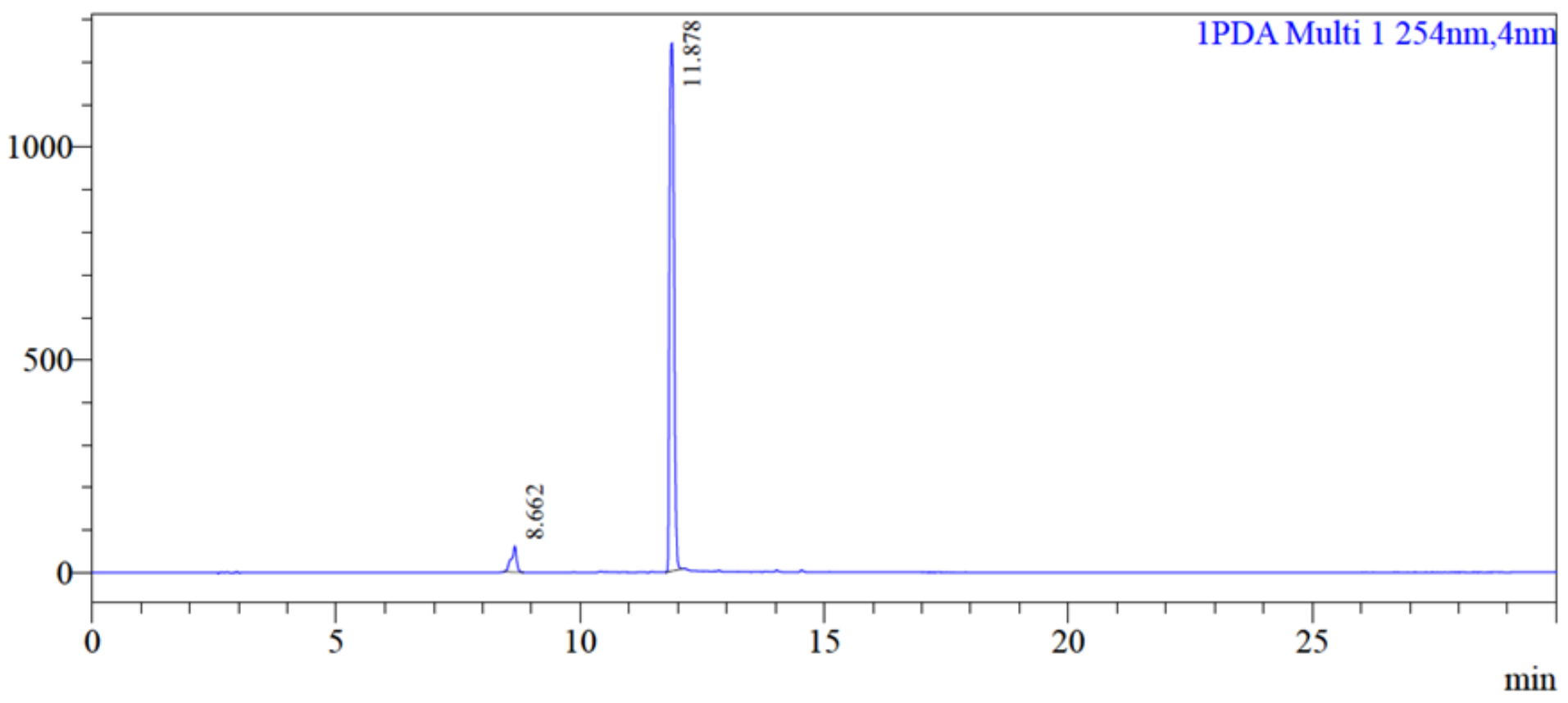

Peak Table

PDA Ch1 $254 \mathrm{~nm}$
\begin{tabular}{|r|r|r|r|r|r|}
\hline Peak $\#$ & Ret. Time & \multicolumn{1}{c|}{ Area } & \multicolumn{1}{c|}{ Height } & Conc. & Name \\
\hline 1 & 8.662 & 460253 & 60506 & 5.427 & \\
\hline 2 & 11.878 & 8020906 & 1241020 & 94.573 & \\
\hline Total & & 8481160 & 1301526 & & \\
\hline
\end{tabular}

Figure S124: RP-HPLC trace of 34. 

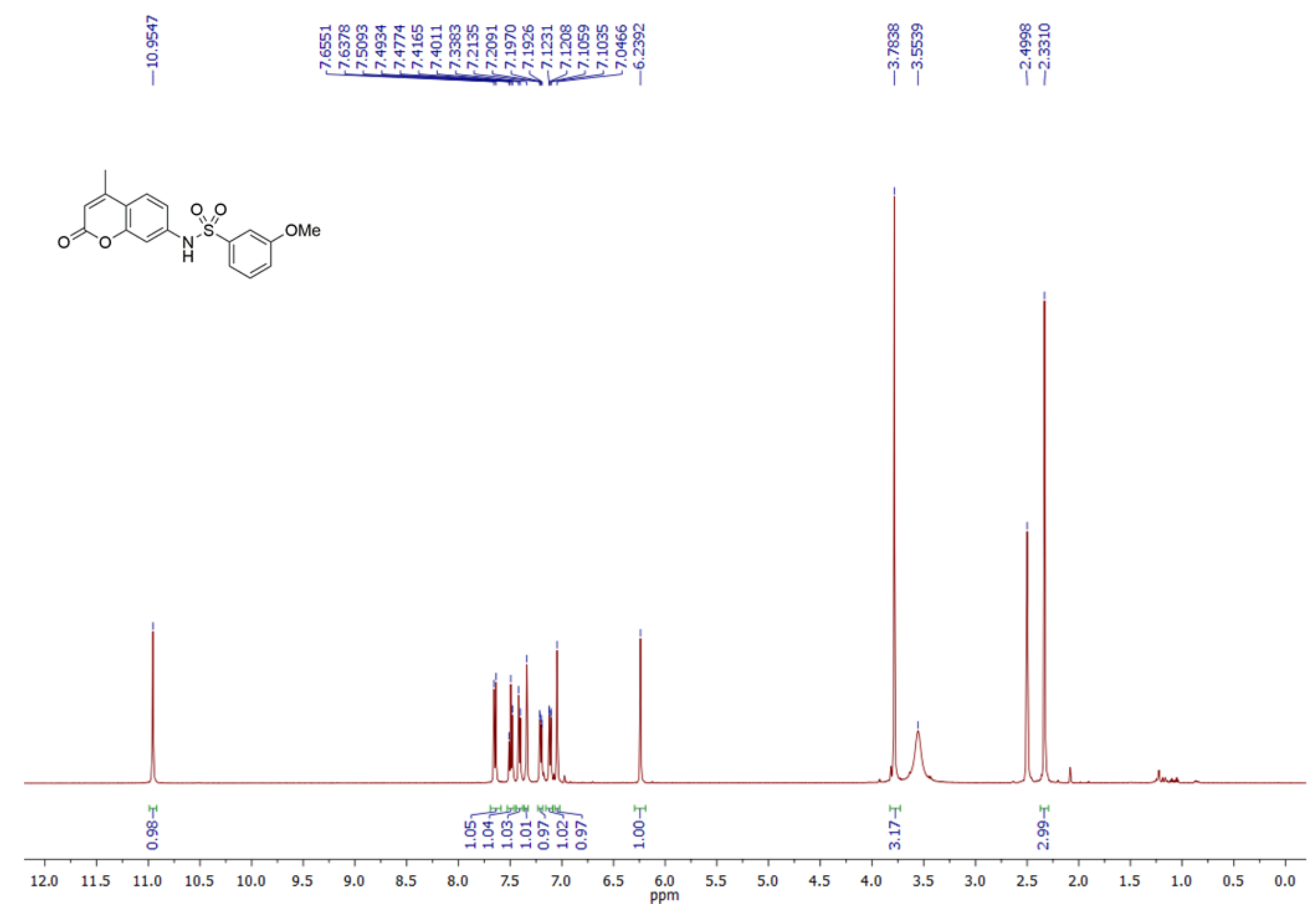

Figure S125: ${ }^{1} \mathrm{H}$ NMR $(500 \mathrm{MHz})$ spectrum of 35 in DMSO- $d_{6}$. 


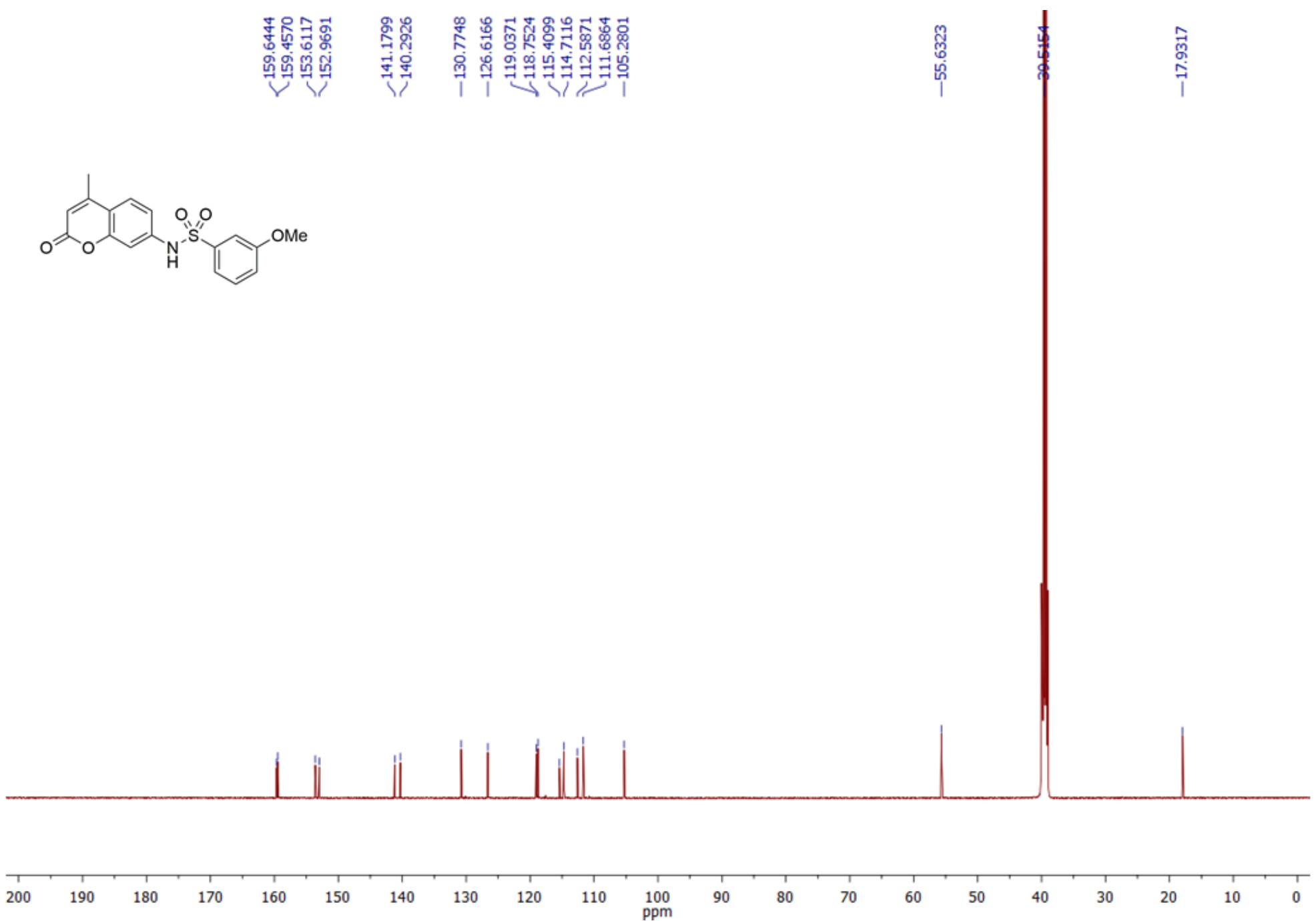

Figure S126: ${ }^{13} \mathrm{C}$ NMR (125 MHz) spectrum of 35 in DMSO- $d_{6}$ 
+TOF MS: 0.1344 to 0.1437 min from Sample 5 (SON04-029B) of SON04-029. wiff
$\mathrm{a}=7.02674672275230960 \mathrm{e}-004$, t0 $=8.46711940862357680 \mathrm{e}-001$ (DuoSpray ())

Max $3.3 e 5 \mathrm{cps}$.

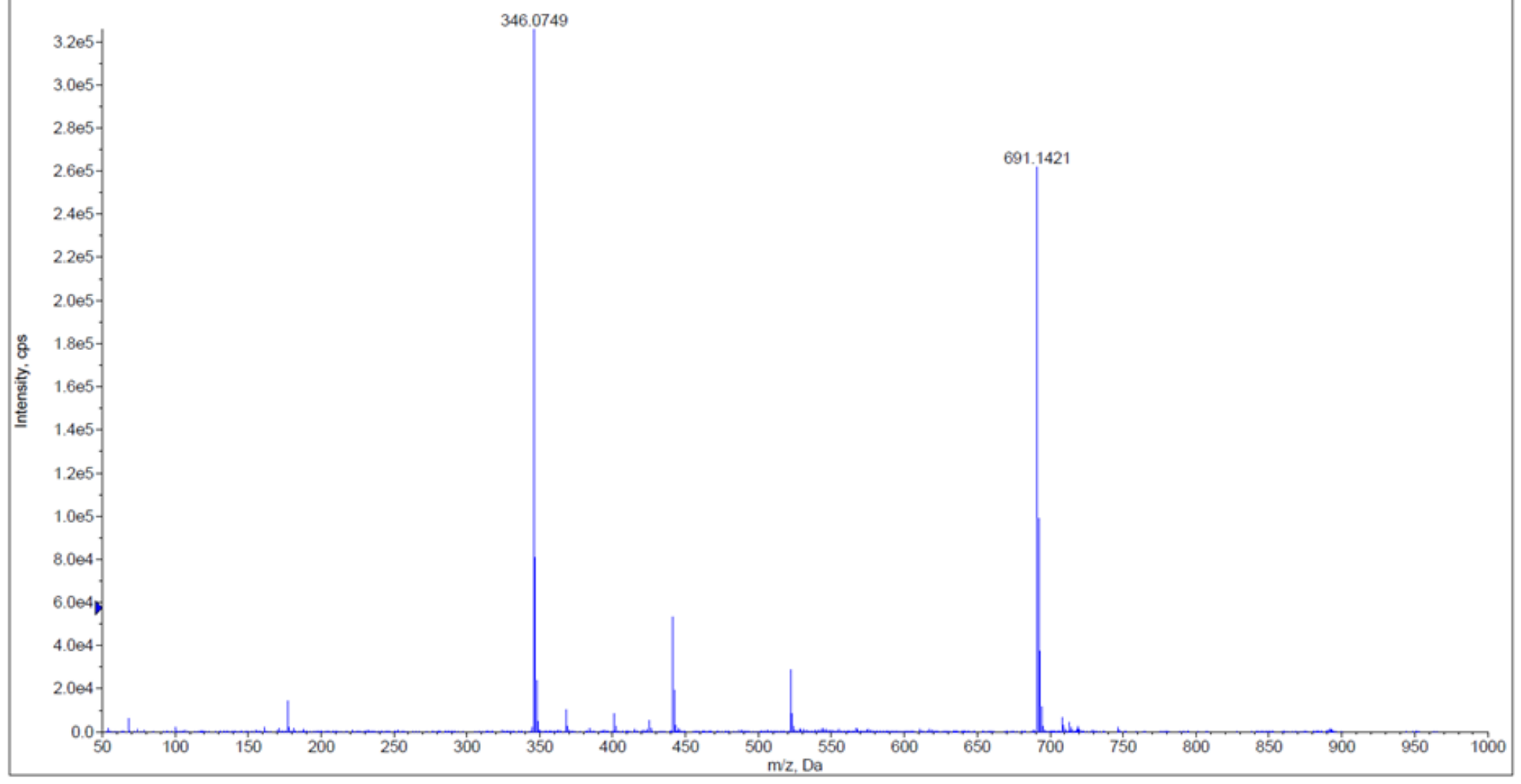

Figure S127: HRMS spectrum for 35 . 
Chromatogram

SON04-029A SON04-029A.led

mAU

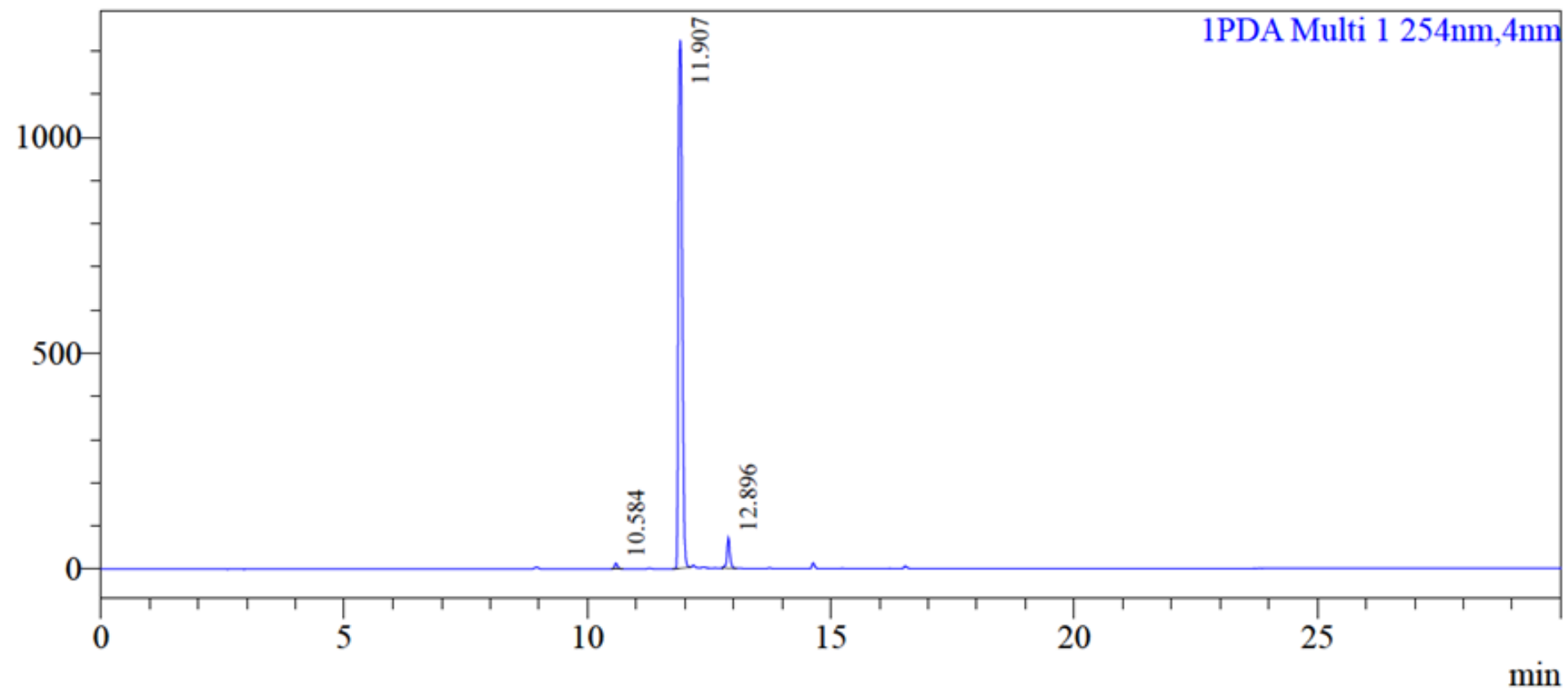

Peak Table

PDA Ch. $254 \mathrm{~nm}$
\begin{tabular}{r|r|r|r|r|l|}
\hline Peak= & Ret. Time & Area & Height & Conc. & Name \\
\hline 1 & 10.584 & 56832 & 13479 & 0.803 & \\
\hline 2 & 11.907 & 6732344 & 1223354 & 95.079 & \\
\hline 3 & 12.896 & 291579 & 69970 & 4.118 & \\
\hline Total & & 7080755 & 1306804 & & \\
\hline
\end{tabular}

Figure S128: RP-HPLC trace for 35. 

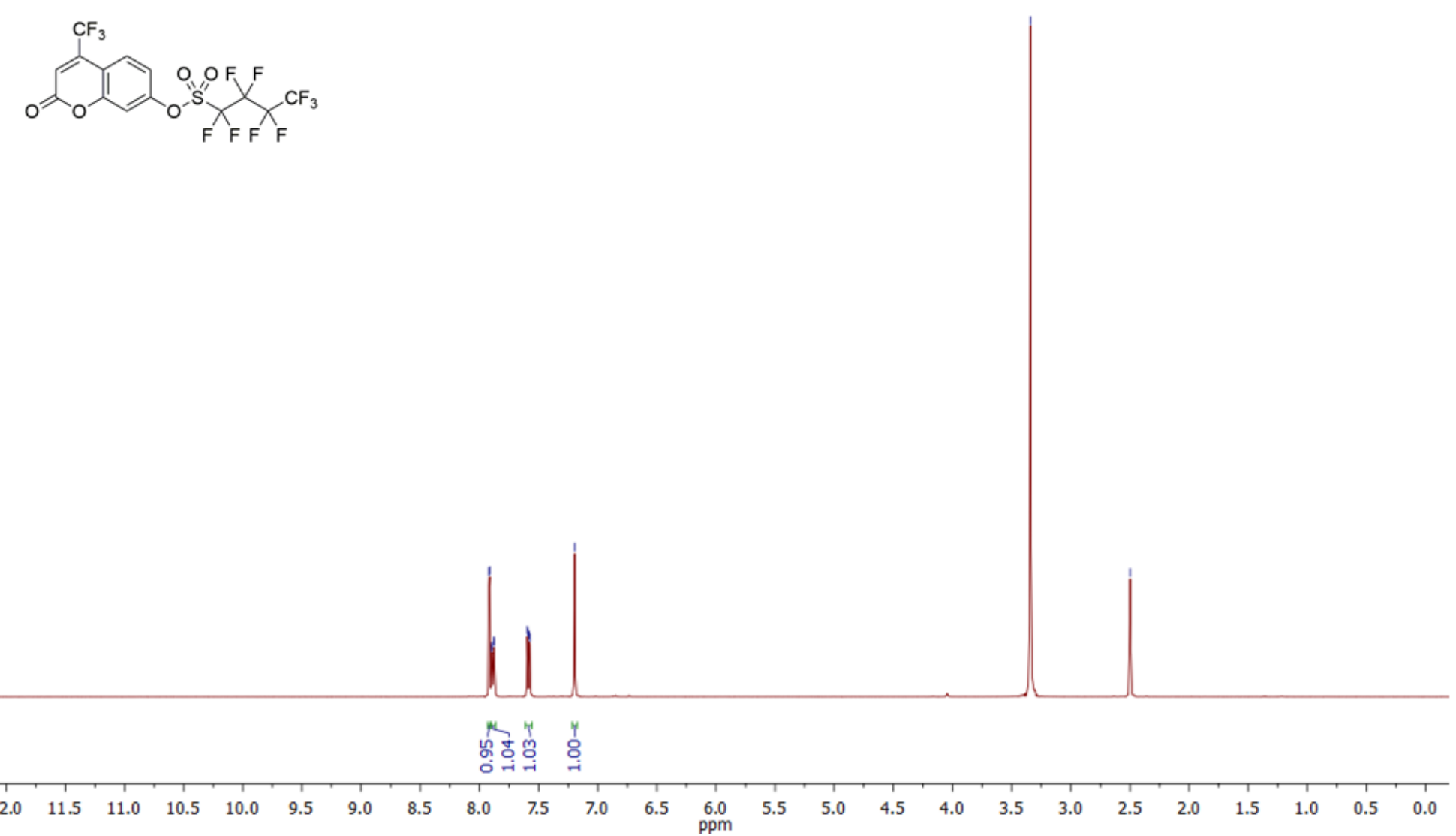

Figure S129: ${ }^{1} \mathrm{H}$ NMR (500 MHz) spectrum of 36 in DMSO- $d_{6}$. 


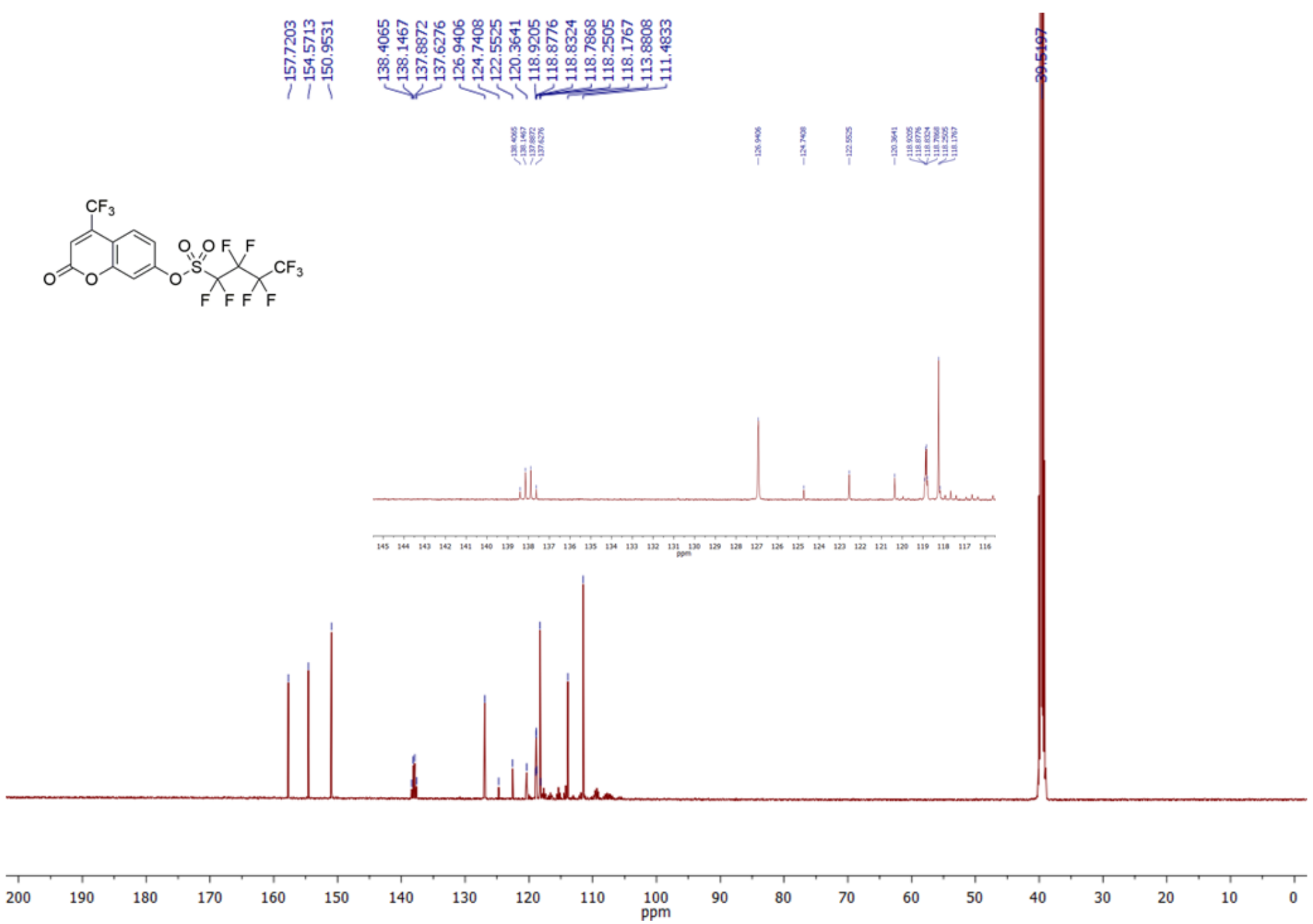

Figure S130: ${ }^{13} \mathrm{C}$ NMR (125 MHz) spectrum of 36 in DMSO- $d_{6}$. Inset: Blown up region (145-116 ppm). 
+TOF MS: Exp 1, 0.0968 to 0.1154 min from Sample 1(SMH11-059A) of SMH11-059A.wiff

Max. 8257.7 cps.

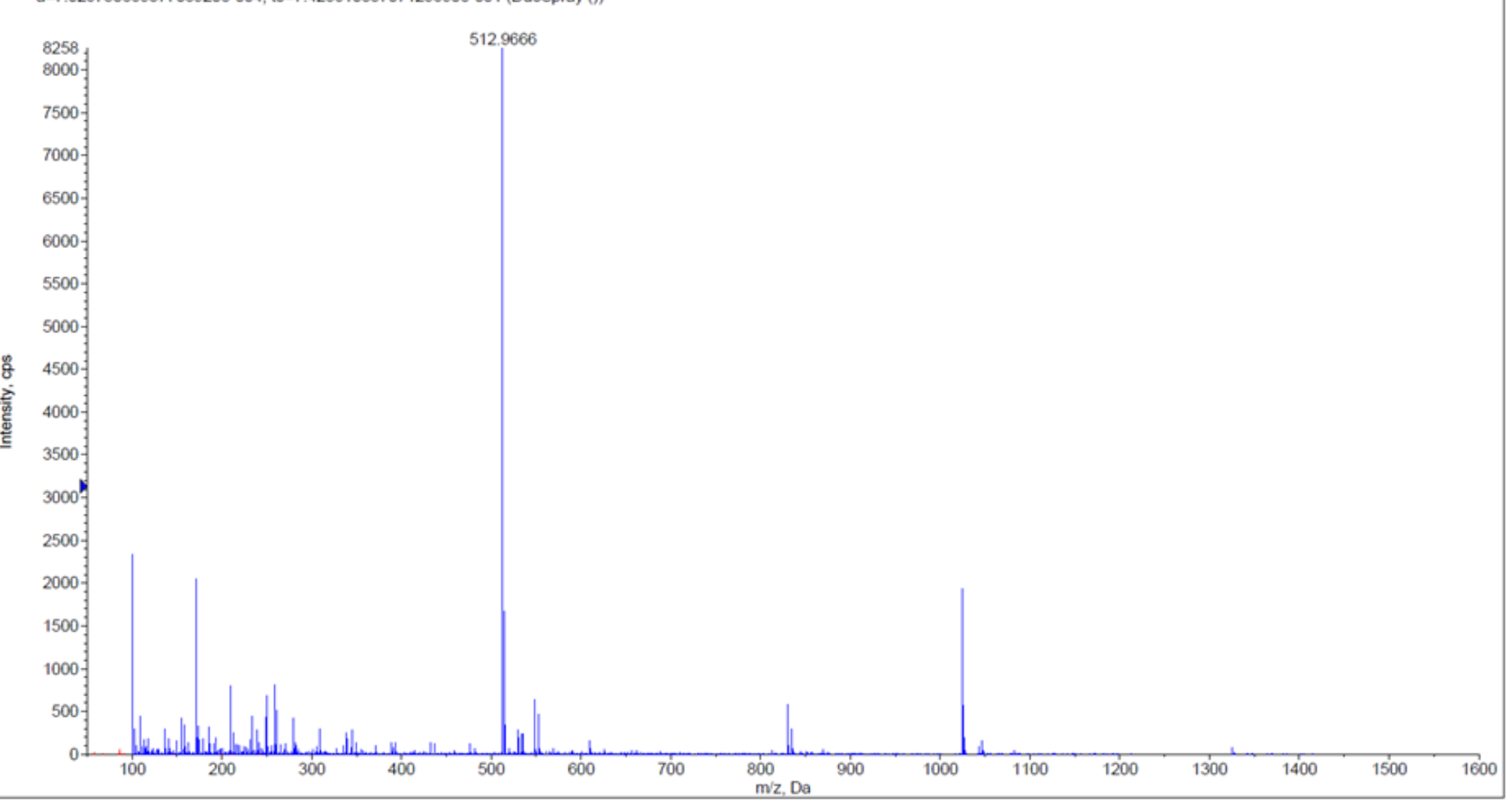

Figure S131: HRMS spectrum for 36 
Chromatogram

SMH11-059A SMH11-059A.lcd

mAU

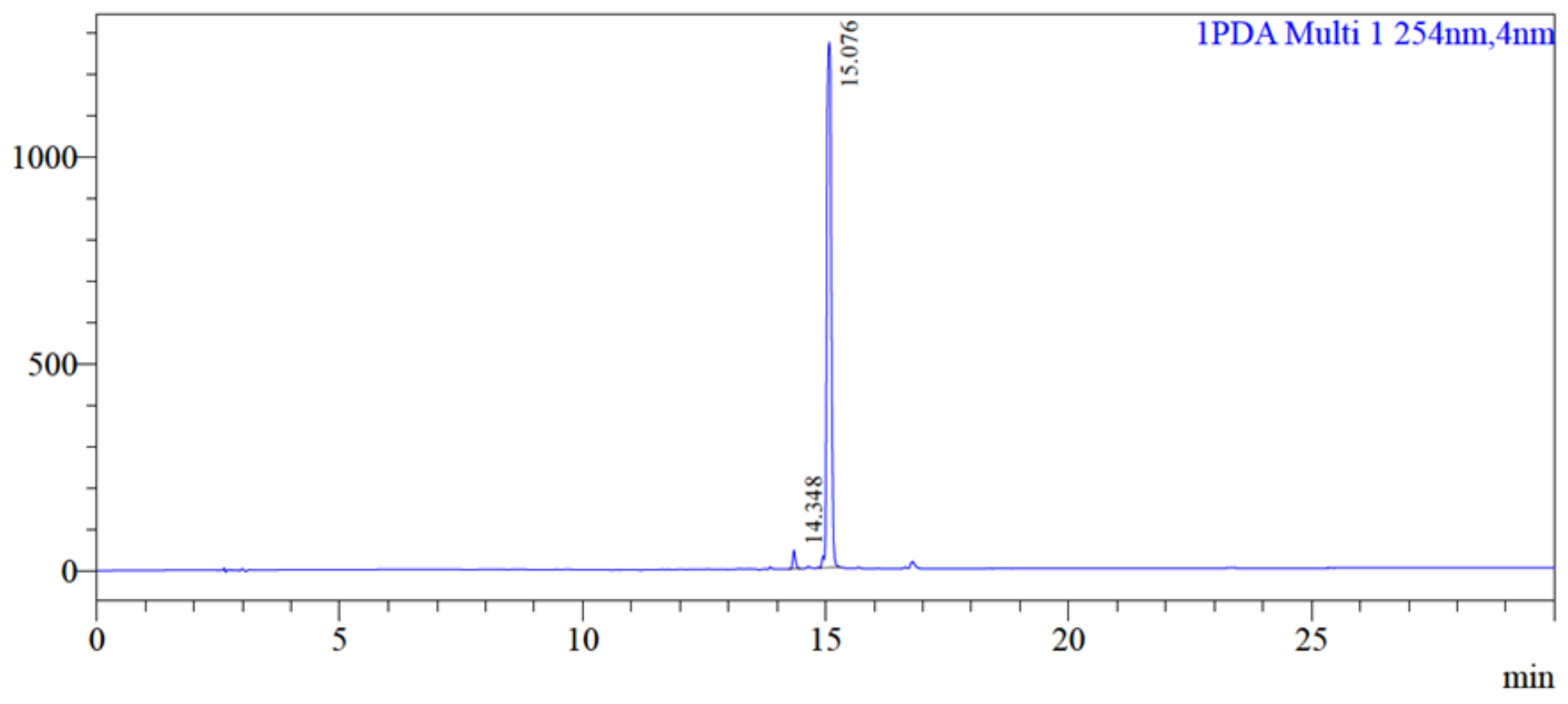

Peak Table

PDA Ch. $254 \mathrm{~nm}$
\begin{tabular}{|r|r|r|r|r|r|}
\hline Peak= & Ret. Time & \multicolumn{1}{|c|}{ Area } & Height & \multicolumn{1}{c|}{ Conc. } & Name \\
\hline 1 & 14.348 & 168756 & 43947 & 2.062 & \\
\hline 2 & 15.076 & 8013746 & 1268118 & 97.938 & \\
\hline Total & & 8182502 & 1312064 & & \\
\hline
\end{tabular}

Figure S132: RP-HPLC trace of $\mathbf{3 6}$. 

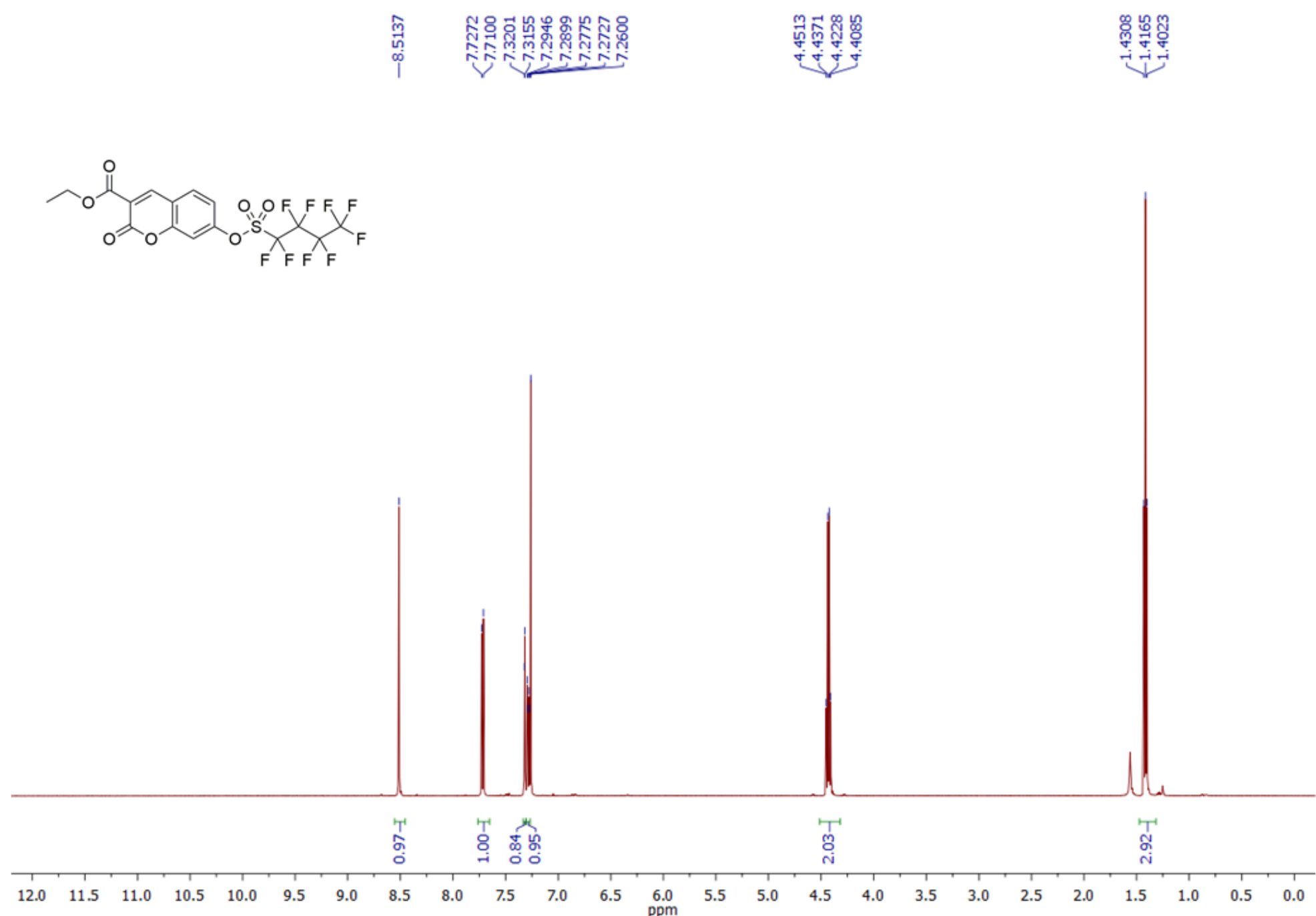

Figure S133: ${ }^{1} \mathrm{H}$ NMR $(500 \mathrm{MHz})$ spectrum of 37 in DMSO- $d_{6}$ 


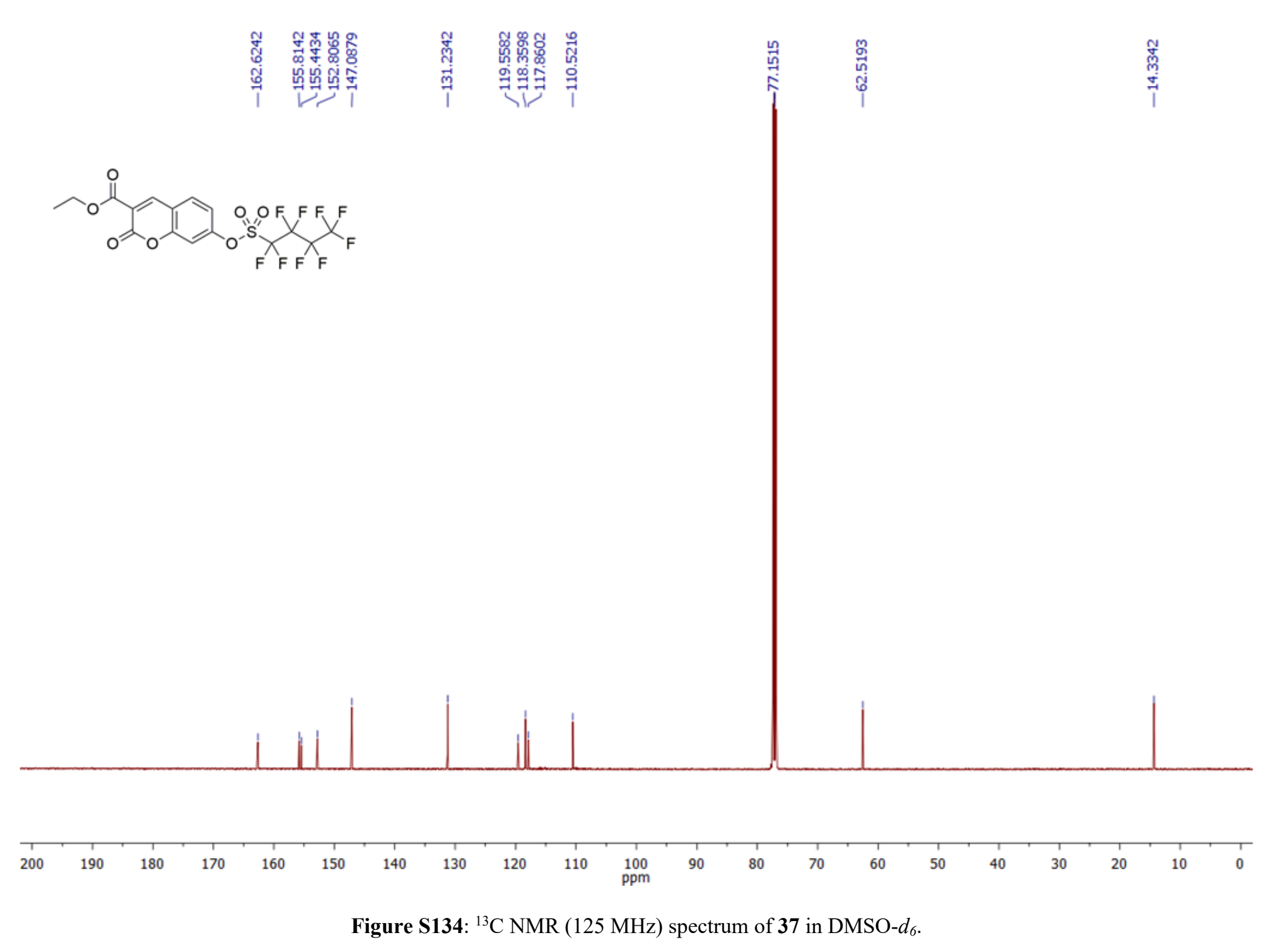


+TOF MS: 0.0925 to $0.0972 \mathrm{~min}$ from Sample 10 (SON03-099C) of SON03-099.wifl

Max. $1.206 \mathrm{cps}$.

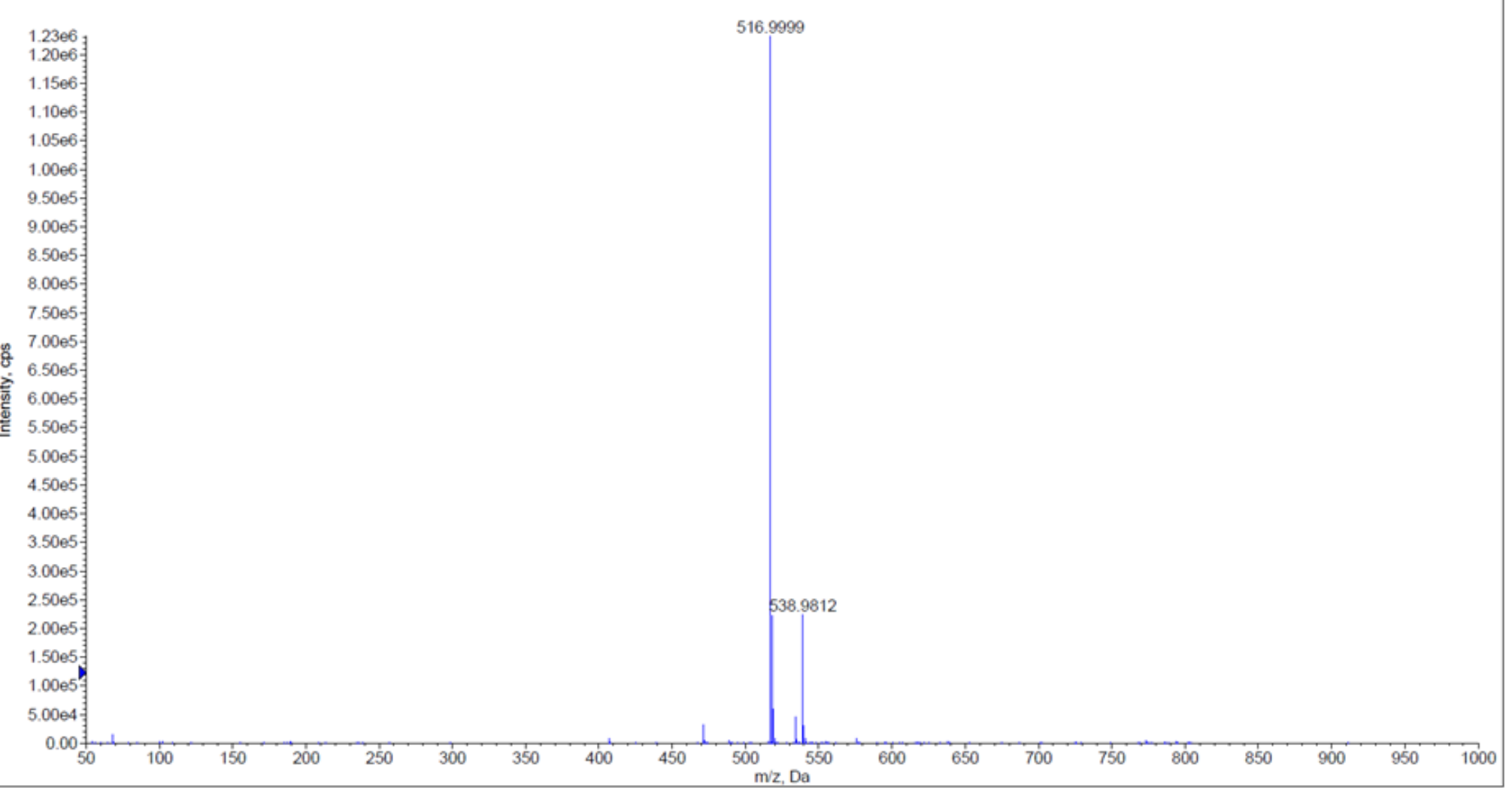

Figure S135: HRMS spectrum for 37. 
Chromatogram

SON03-099C SON03-099C.led

mAU

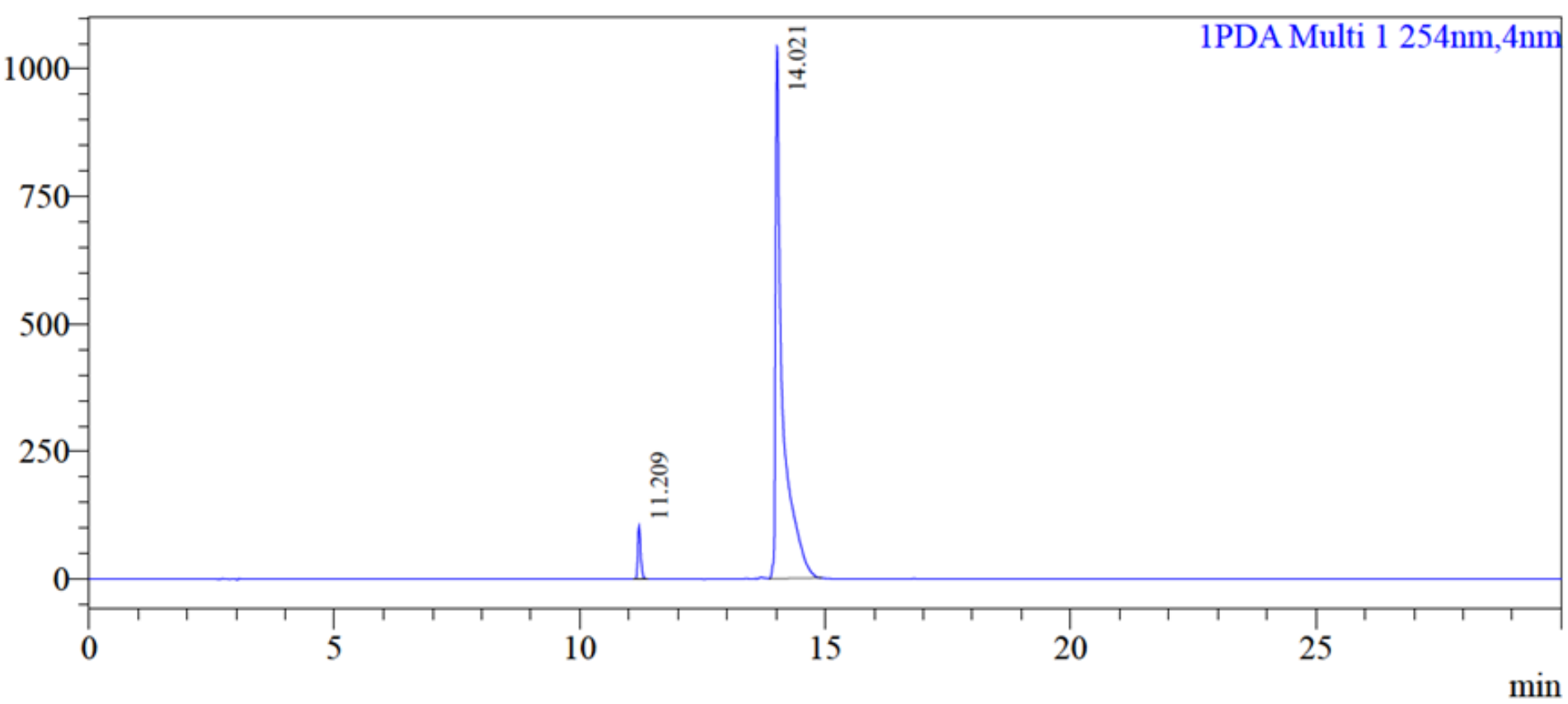

Peak Table

PDA Ch1 $254 \mathrm{~nm}$
\begin{tabular}{|r|r|r|r|r|r|}
\hline Peak $\#$ & Ret. Time & \multicolumn{1}{|c|}{ Area } & \multicolumn{1}{c|}{ Height } & \multicolumn{1}{c|}{ Conc. } & Name \\
\hline 1 & 11.209 & 425525 & 103657 & 3.888 & \\
\hline 2 & 14.021 & 10518902 & 1042903 & 96.112 & \\
\hline Total & & 10944426 & 1146560 & & \\
\hline
\end{tabular}

Figure S136: RP-HPLC trace for 37. 


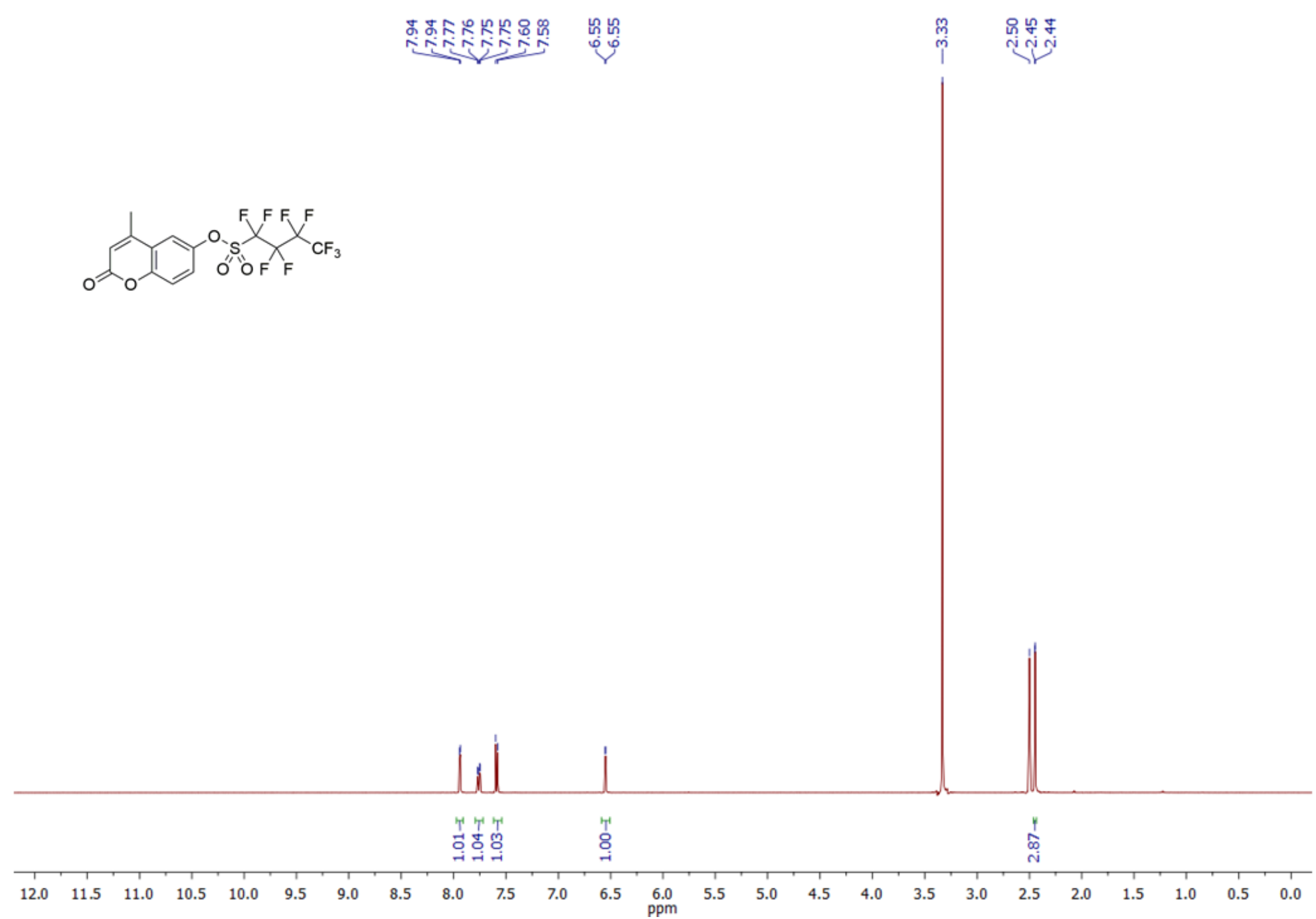

Figure S137: ${ }^{1} \mathrm{H}$ NMR (500 MHz) spectrum of 38 in DMSO- $d_{6}$. 


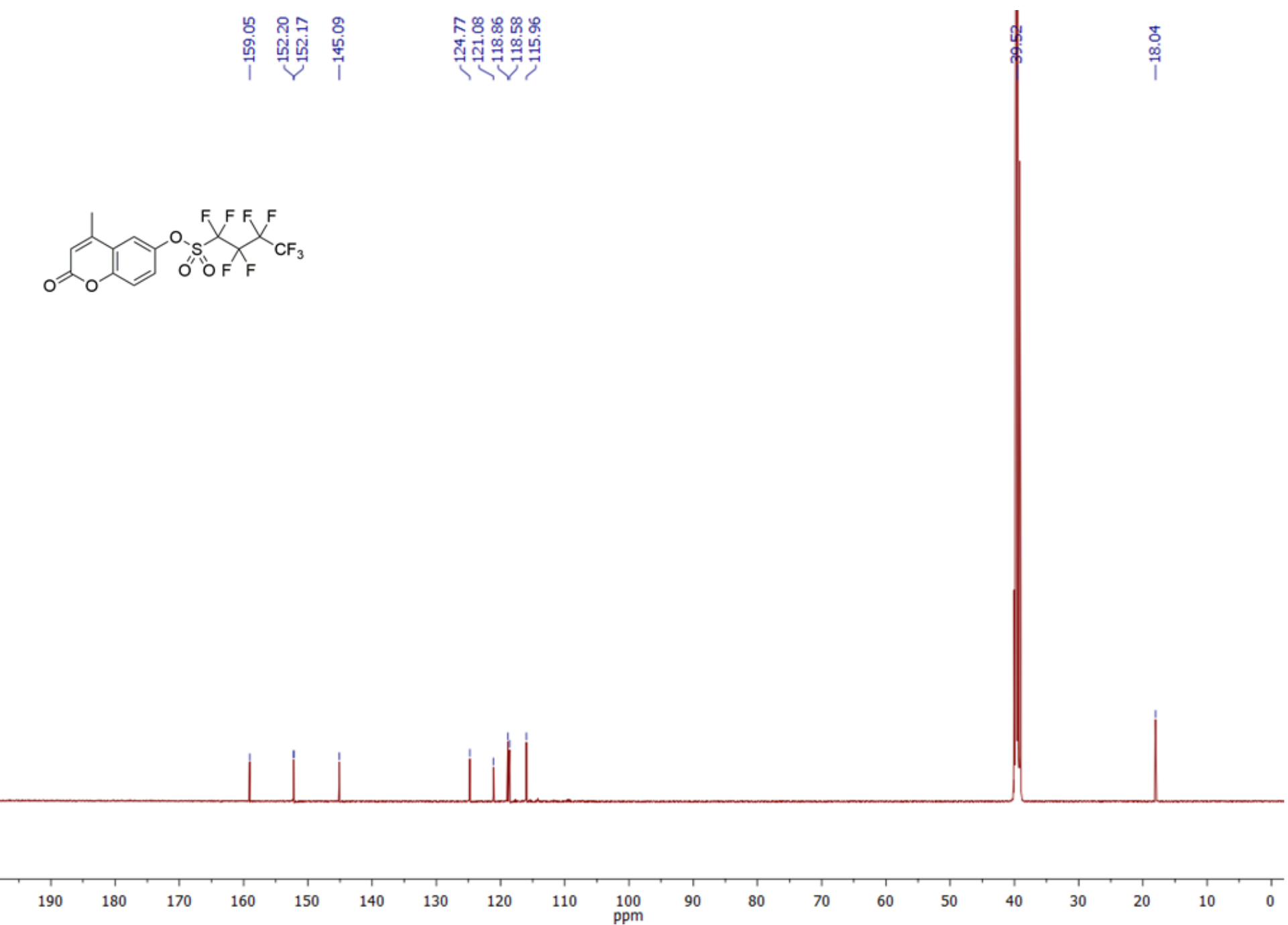

Figure S138: ${ }^{13} \mathrm{C}$ NMR $(125 \mathrm{MHz})$ spectrum of 38 in DMSO- $d_{6}$. 
+TOF MS: Exp 1, 0.0969 to $0.1154 \mathrm{~min}$ from Sample 1(SMH11-069A) of SMH11-069A wiff
$\mathrm{a}=7.02658808542675190 \theta-004,10=7.219876610982957100-001$ (DuoSpray 0 )

Max. $2.8 \mathrm{es} \mathrm{cps}$.

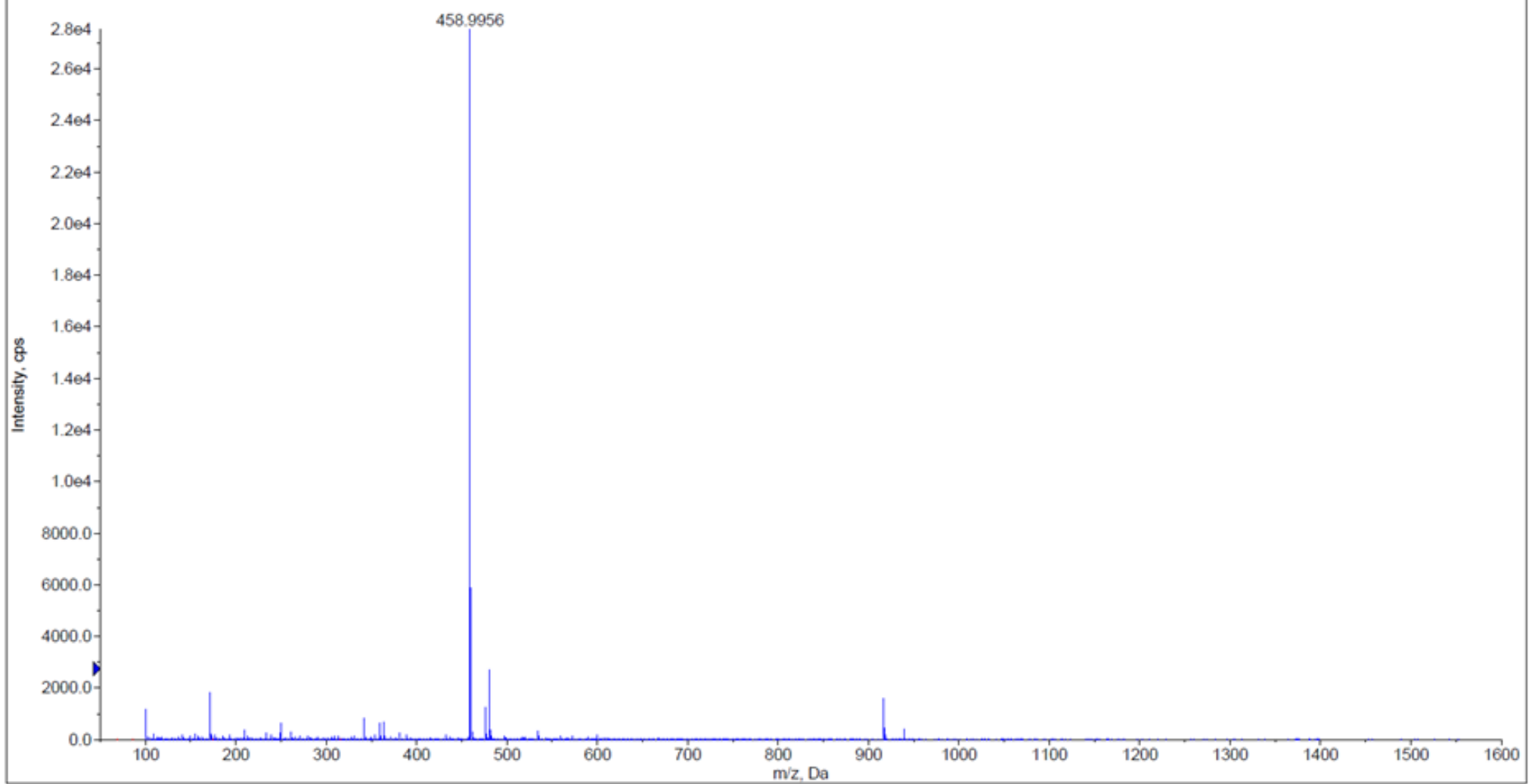

Figure S139: HRMS spectrum of 38. 
Chromatogram

mAU

SMH11-069A SMH11-069A.led

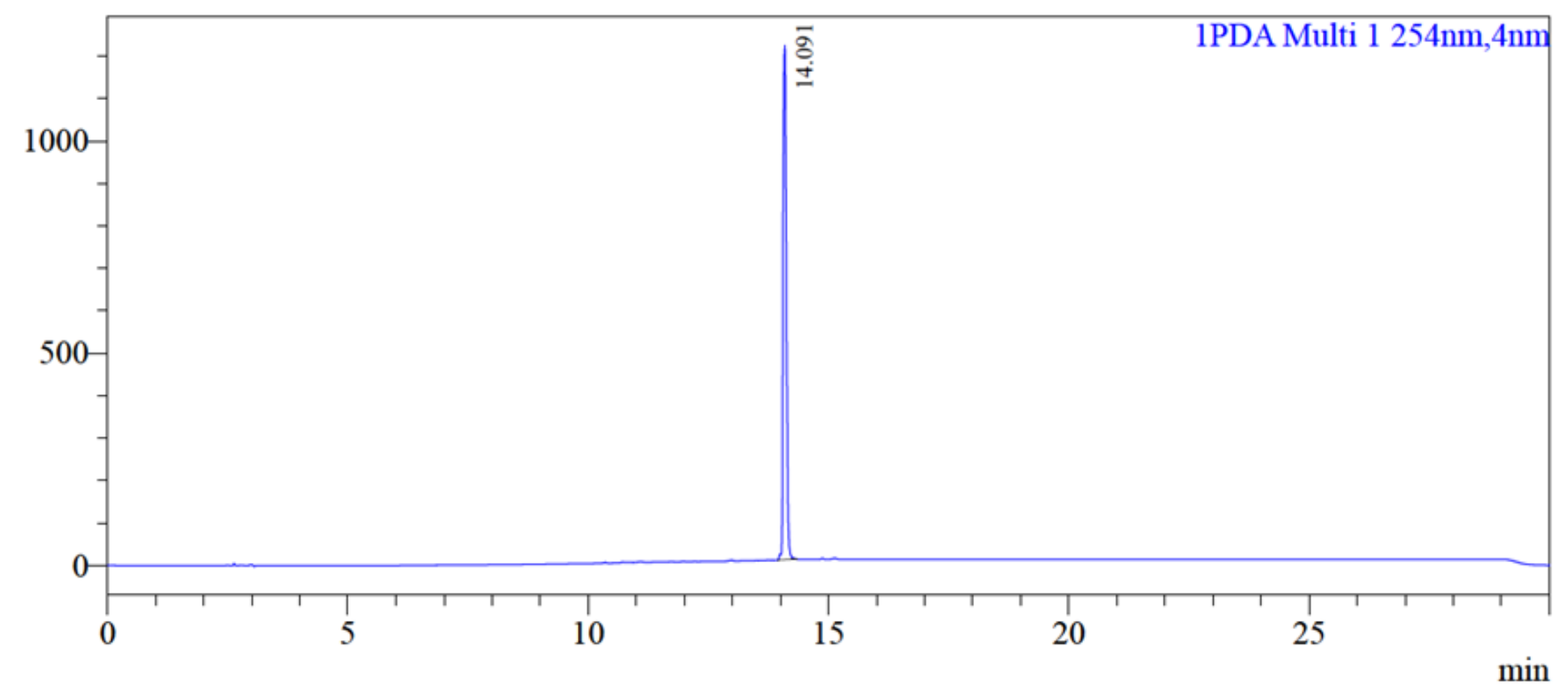

Peak Table

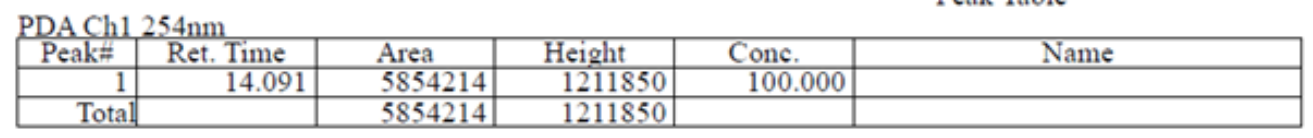

Figure S140: RP-HPLC trace of $\mathbf{3 8}$. 


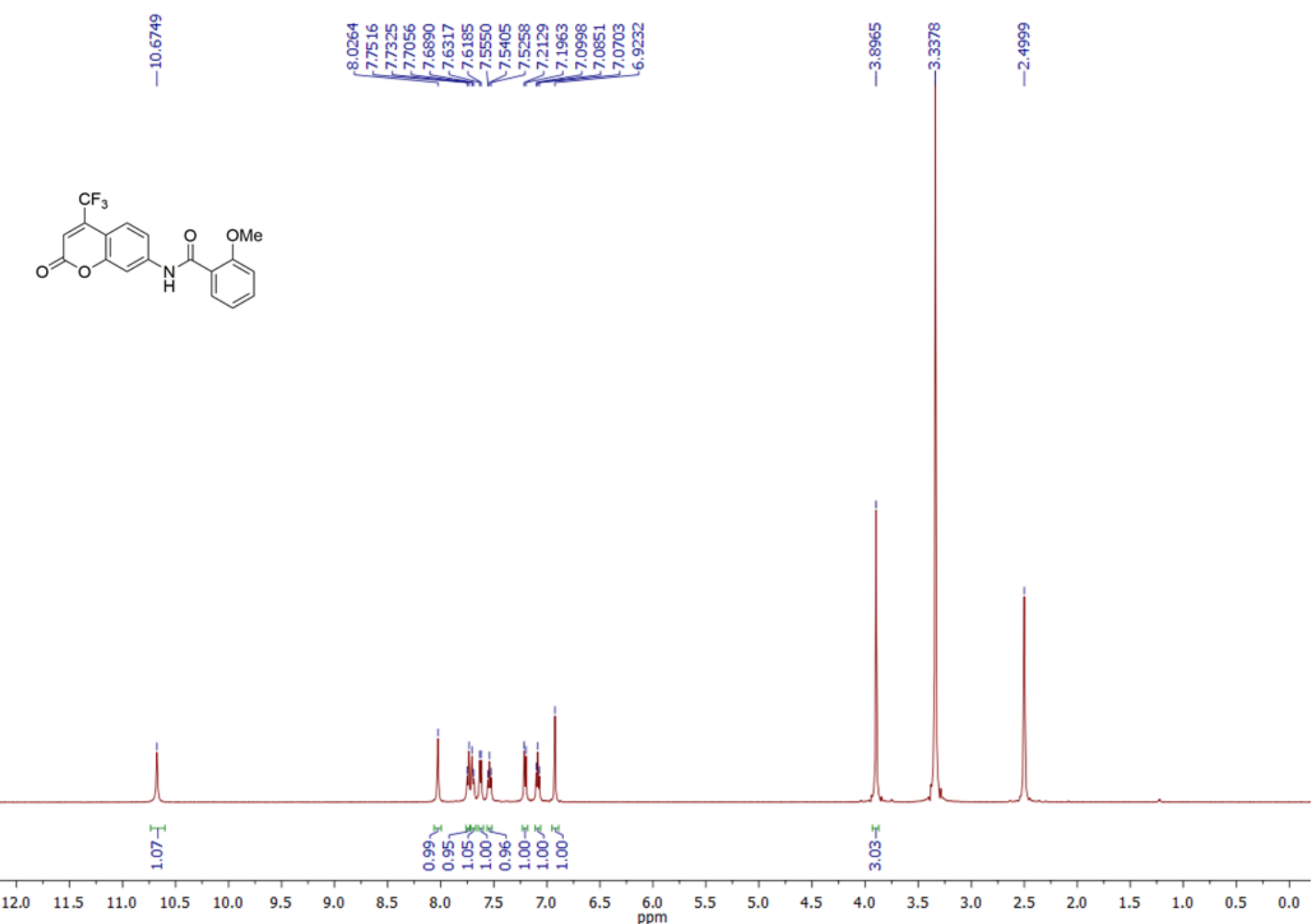

Figure S141: ${ }^{1} \mathrm{H}$ NMR (500 MHz) spectrum of 39 in DMSO- $d_{6}$. 
苗

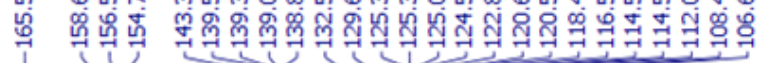

离 क

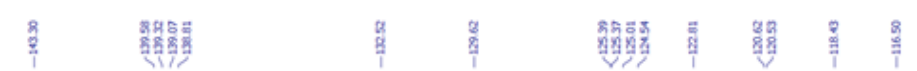
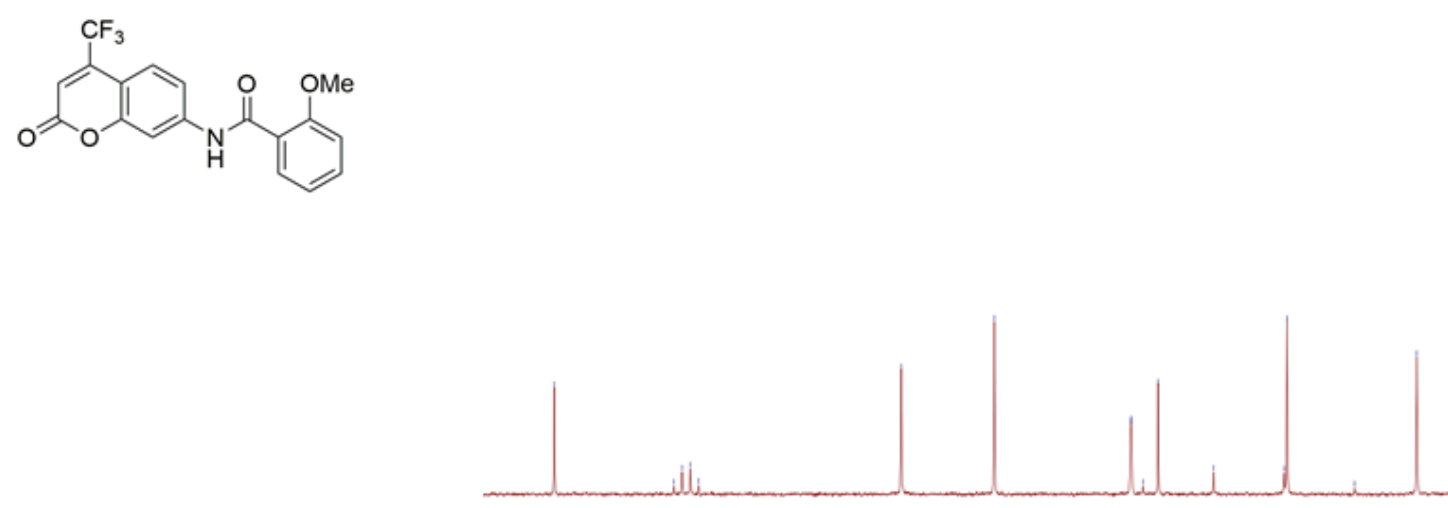

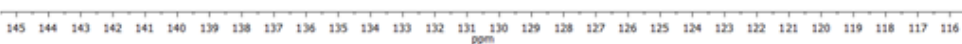

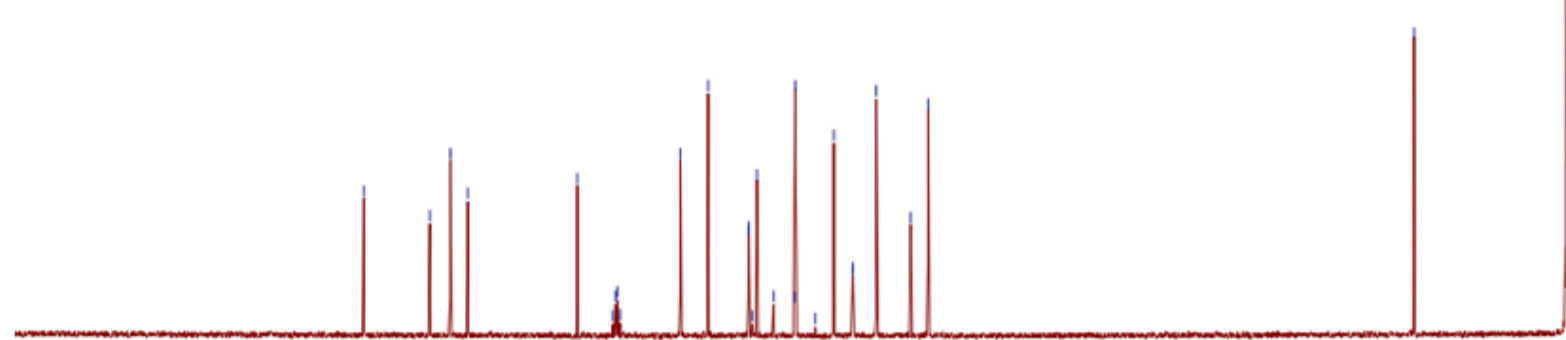

$\begin{array}{rrr} & 1 & \\ 200 & 190 & 180\end{array}$

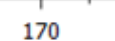

$160 \quad 150$

$140 \quad 130$
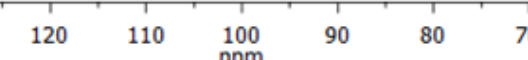

igure S142: ${ }^{13} \mathrm{C}$ NMR $(125 \mathrm{MHz})$ spectrum of 39 in DMSO- $d_{6}$. Inset: Blown up region (145-116 ppm). 
+TOF MS: Exp 1, 0.0875 to 0.1061 min from Sample 2 (SMH11-065A) of SMH11-065A.wiff

Max $4.404 \mathrm{cps}$. a $7.026713223627168000-004,10=7.525936730891346100-001$ (Duospray ())

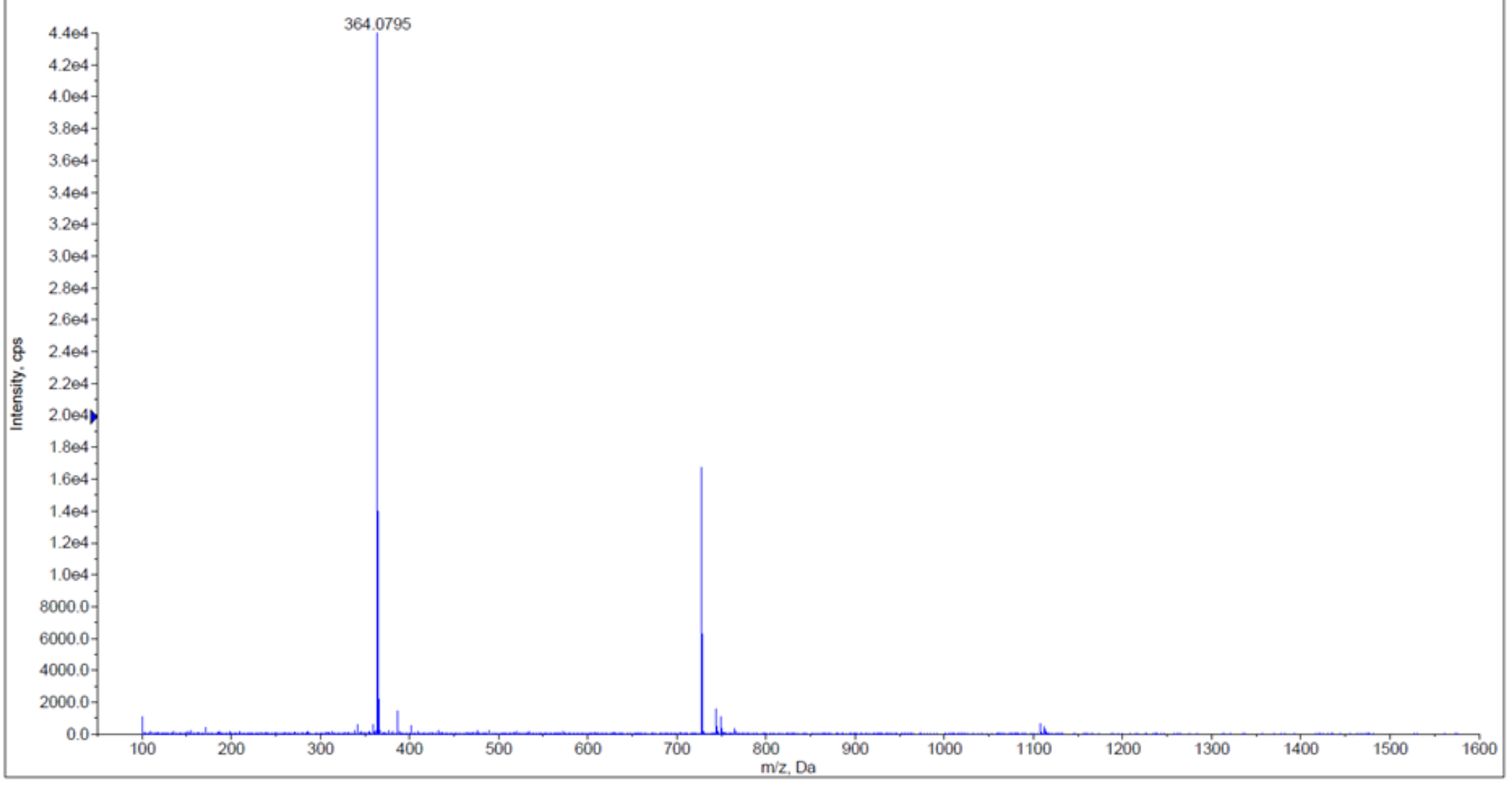

Figure S143: HRMS spectrum of 39. 
Chromatogram

SMH11-065A SMH11-065A.lcd

mAU

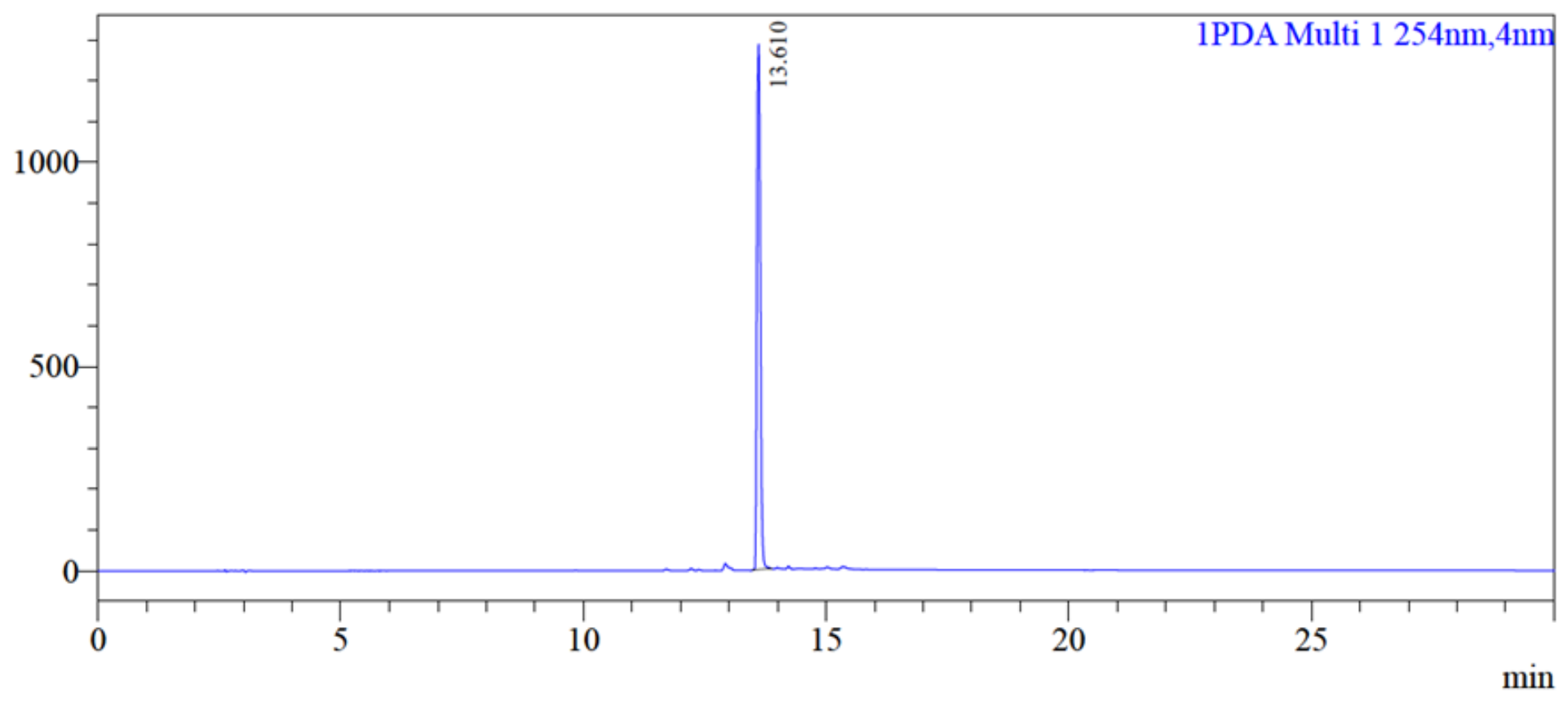

Peak Table

PDA Chl $254 \mathrm{~nm}$
\begin{tabular}{|r|r|r|r|r|r|}
\hline Peak: $\#$ & Ret. Time & Area & Height & Conc. & Name \\
\hline 1 & 13.610 & 6352355 & 1285952 & 100.000 & \\
\hline Total & & 6352355 & 1285952 & & \\
\hline
\end{tabular}

Figure S144: RP-HPLC trace of 39. 


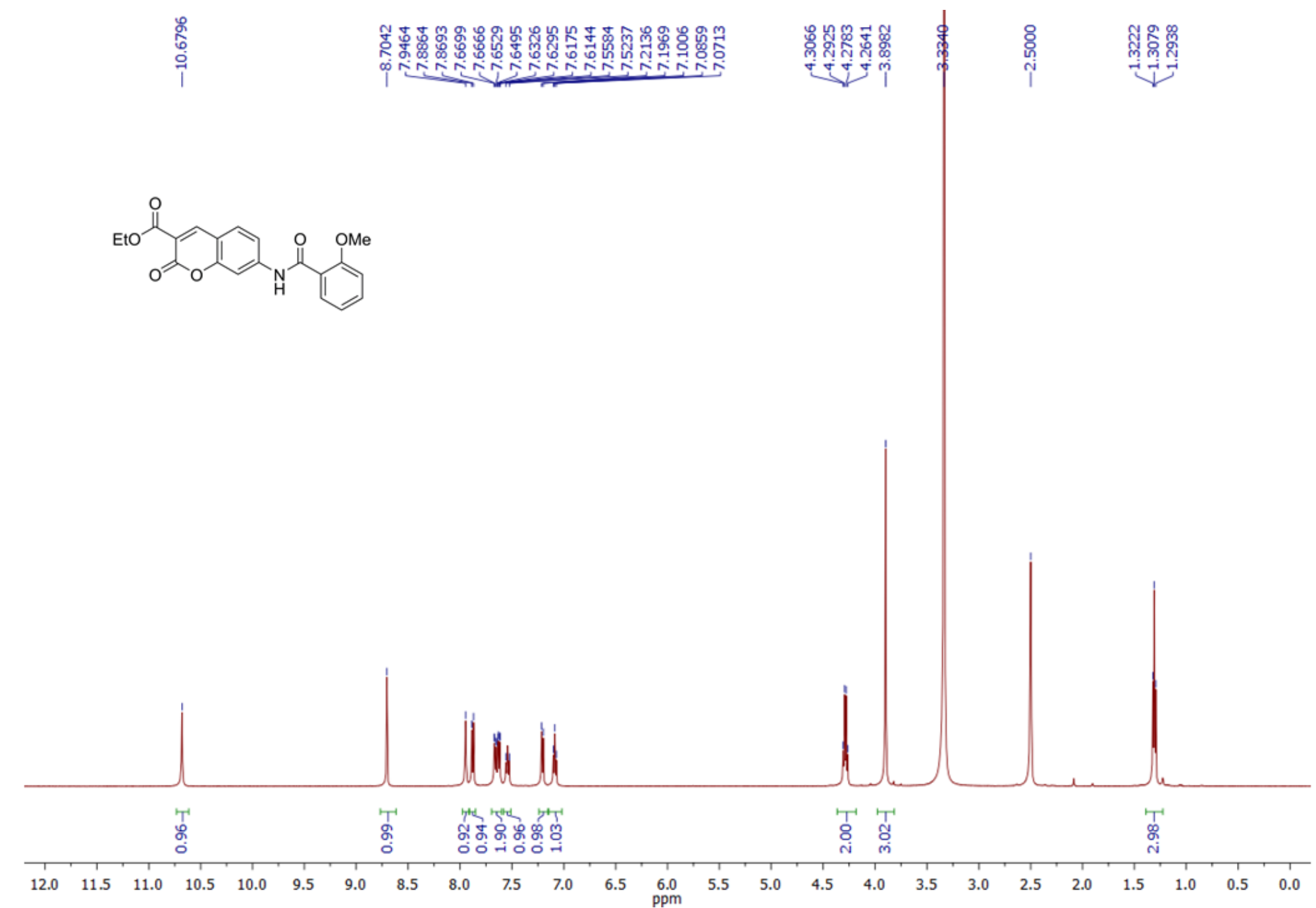

Figure S145: ${ }^{1} \mathrm{H}$ NMR $(500 \mathrm{MHz})$ spectrum of 40 in DMSO- $d_{6}$. 


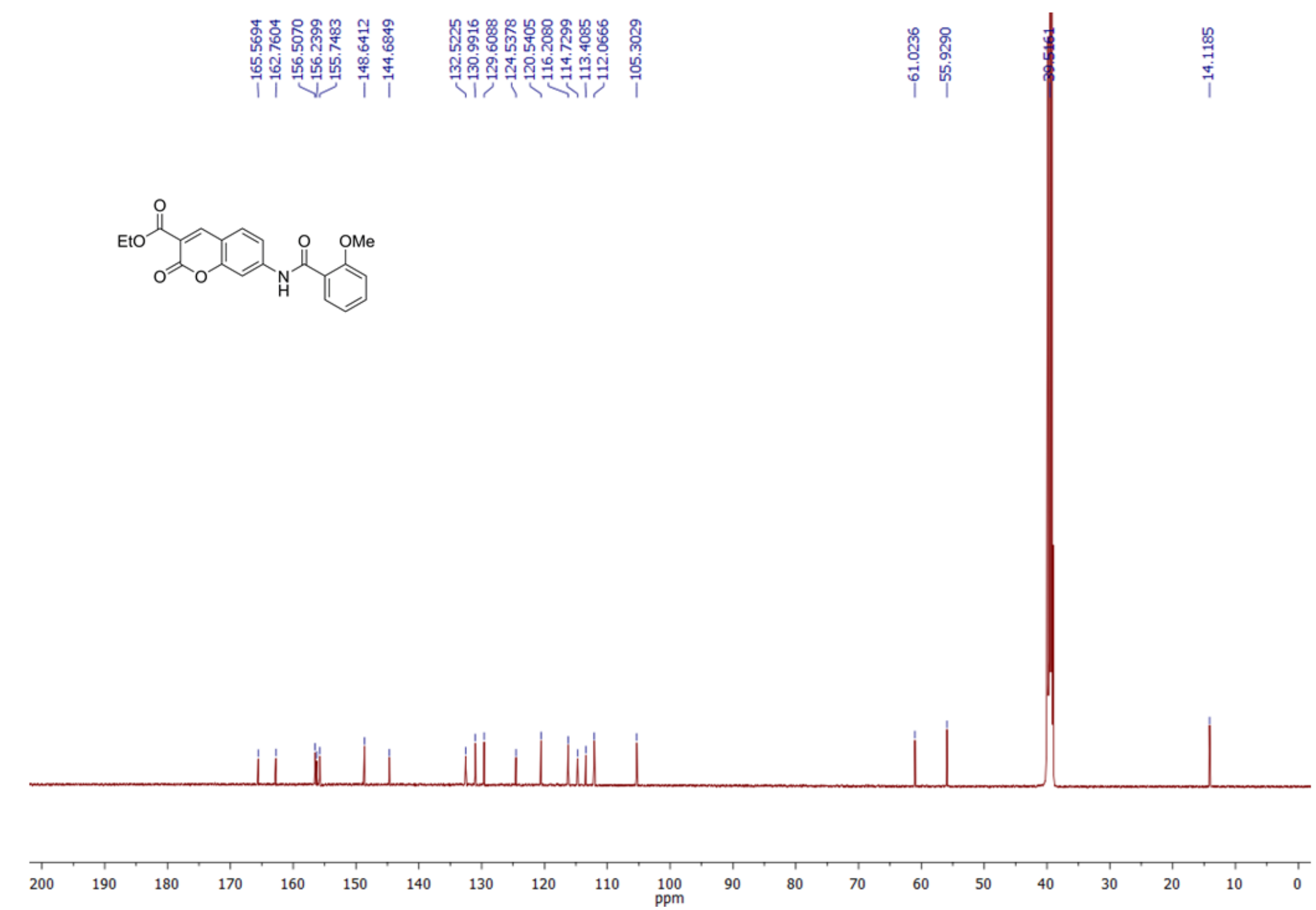

Figure S146: ${ }^{13} \mathrm{C}$ NMR $(125 \mathrm{MHz})$ spectrum of 40 in DMSO- $d_{6}$. 
+TOF MS: 0.0879 to 0.0972 min from Sample 4 (SON03-103A) of SON03-103 wiff

Max. $1.105 \mathrm{cps}$.

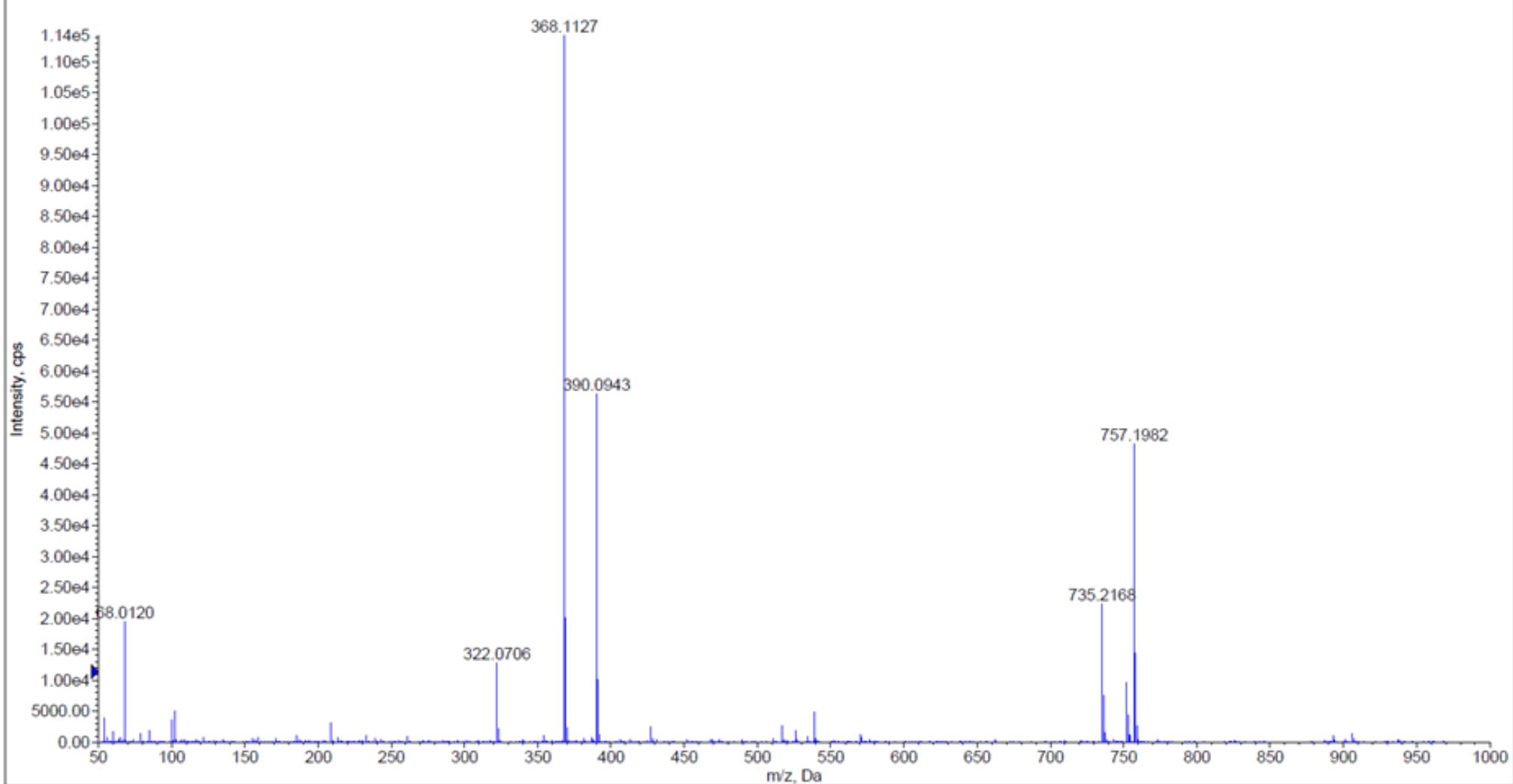

Figure S147: HRMS spectrum for $\mathbf{4 0}$ 
Chromatogram

SON03-103B SON03-103B.lcd

mAU

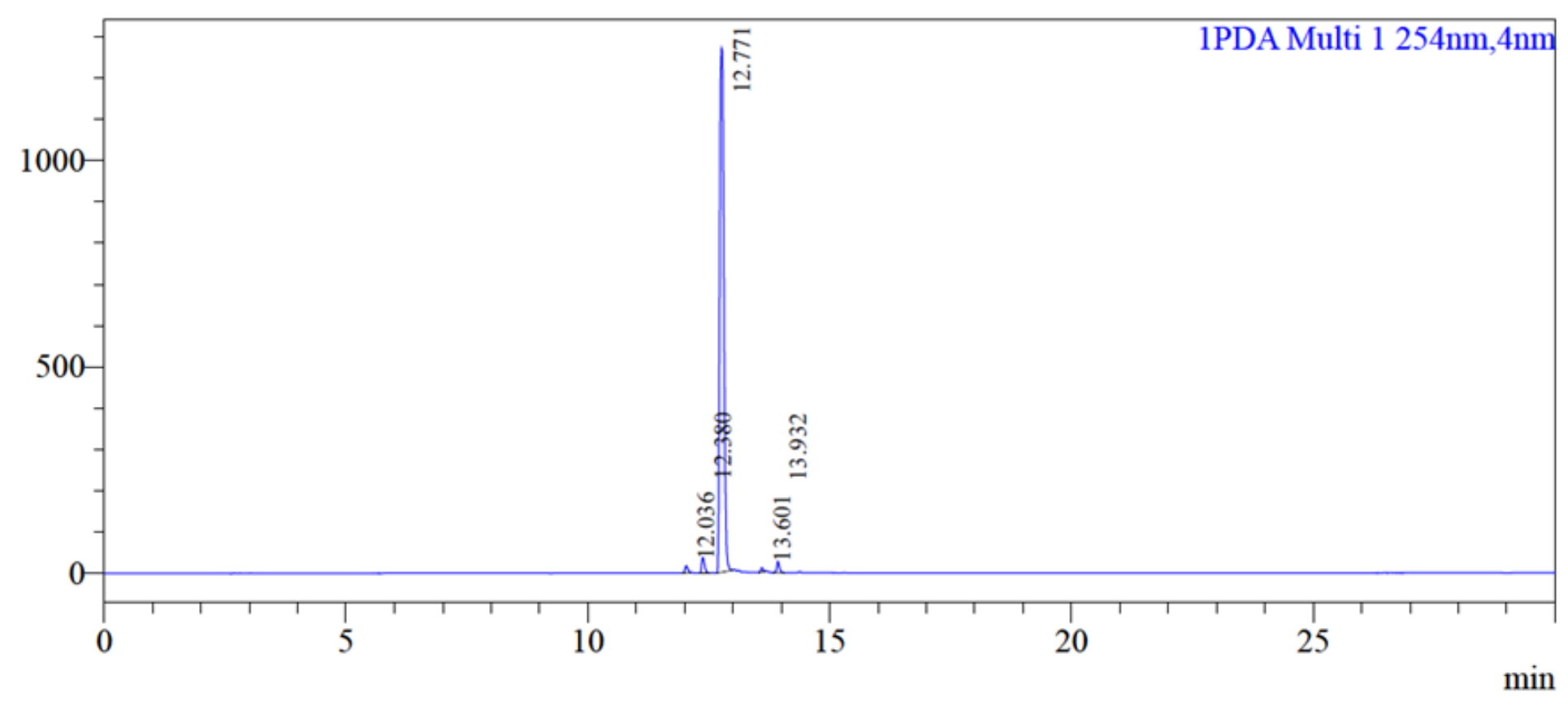

Peak Table

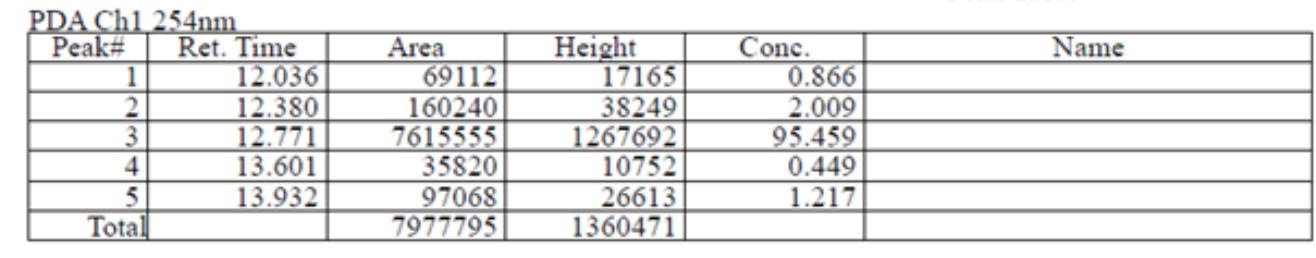

Figure S148: RP-HPLC trace for 40. 


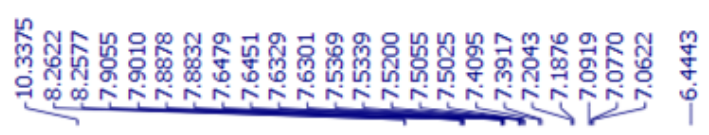
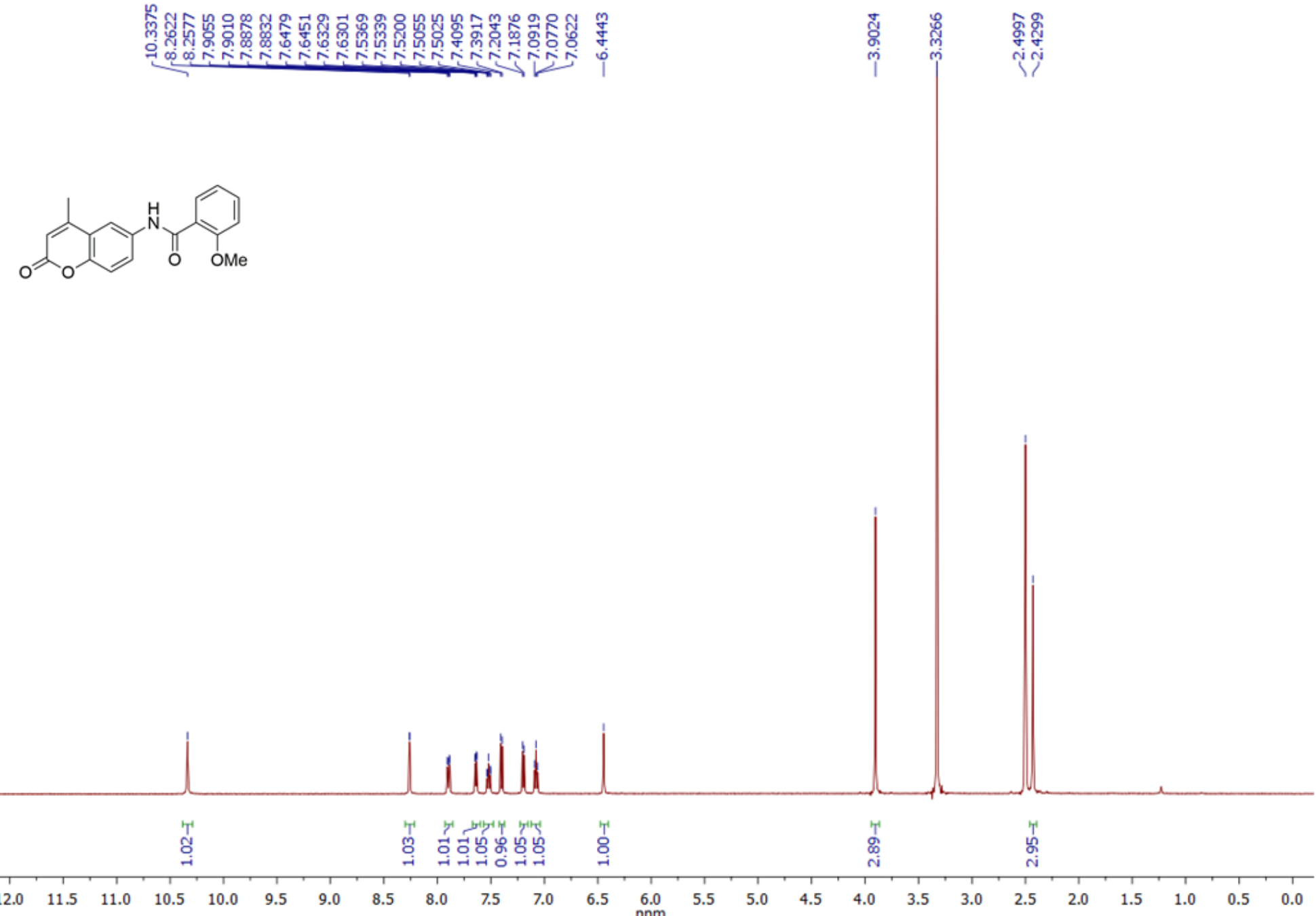

Figure S149: ${ }^{1} \mathrm{H}$ NMR $(500 \mathrm{MHz})$ spectrum of 41 in DMSO- $d_{6}$ 


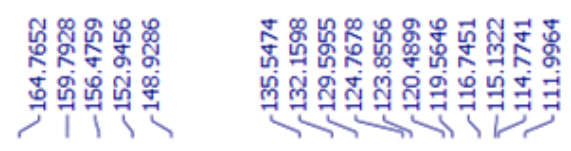
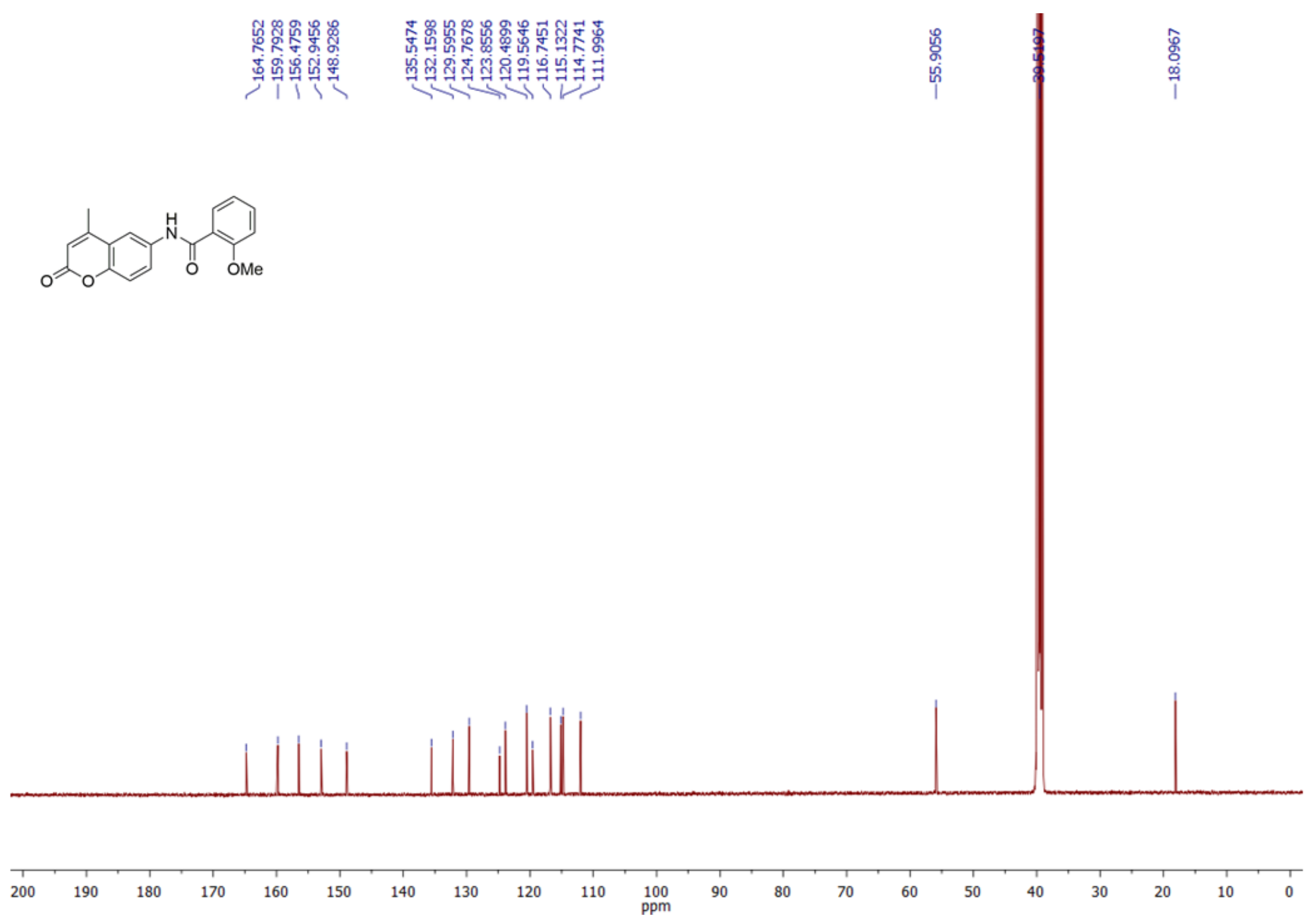

Figure S150: ${ }^{13} \mathrm{C}$ NMR (125 MHz) spectrum of 41 in DMSO- $d_{6}$.

$\mathrm{S} 151$ 
- +TOF MS: Exp 1, 0.3935 to 0.4212 min from Sample 1(SMH11-073A) of SMH11-073A.wiff different calibrations (DuoSpray ())

Max. $6636.5 \mathrm{cps}$.

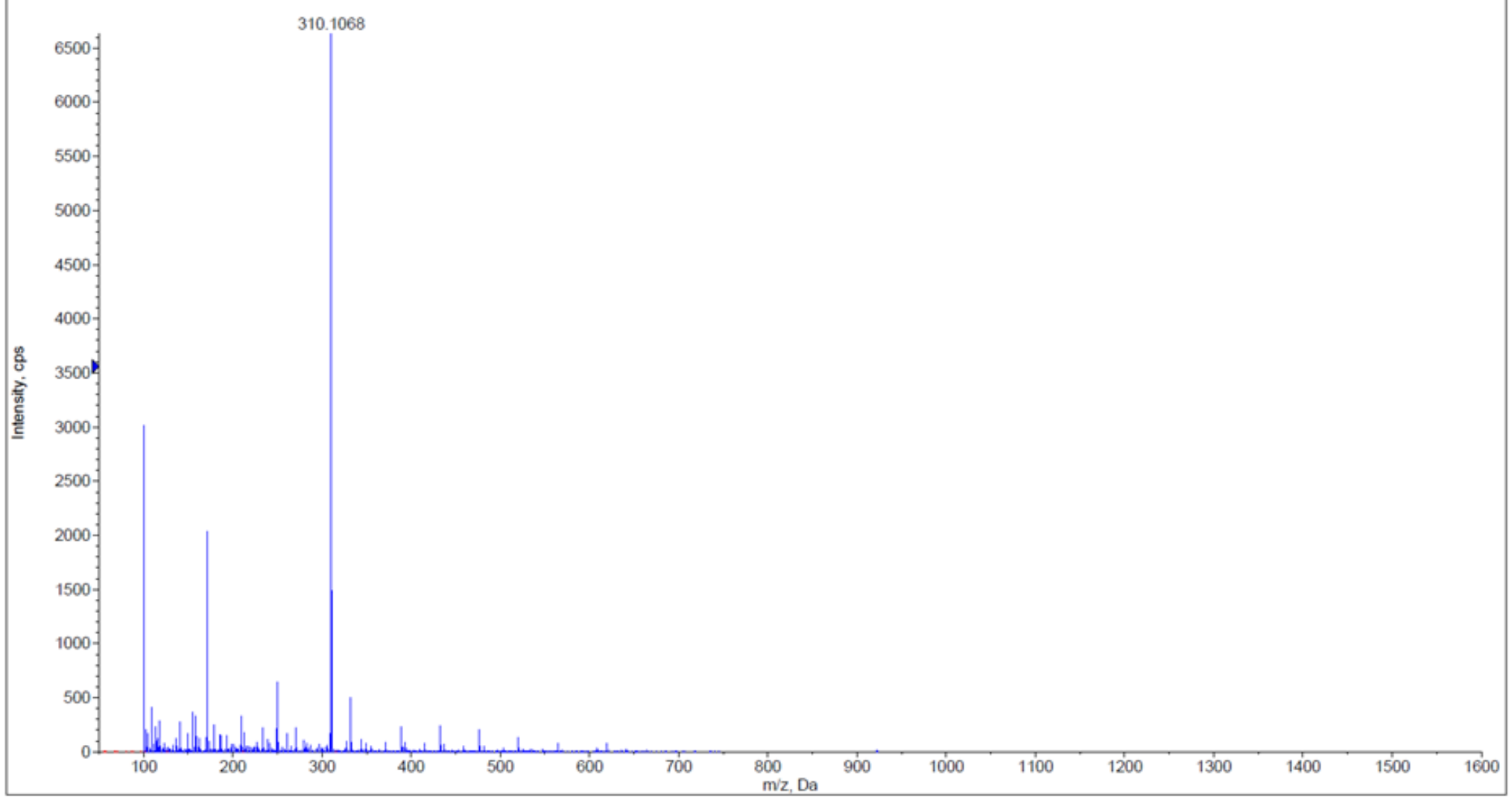

Figure S151: HRMS spectrum of $\mathbf{4 1}$. 
Chromatogram

SMH11-073A SMH11-073A.led

mAU

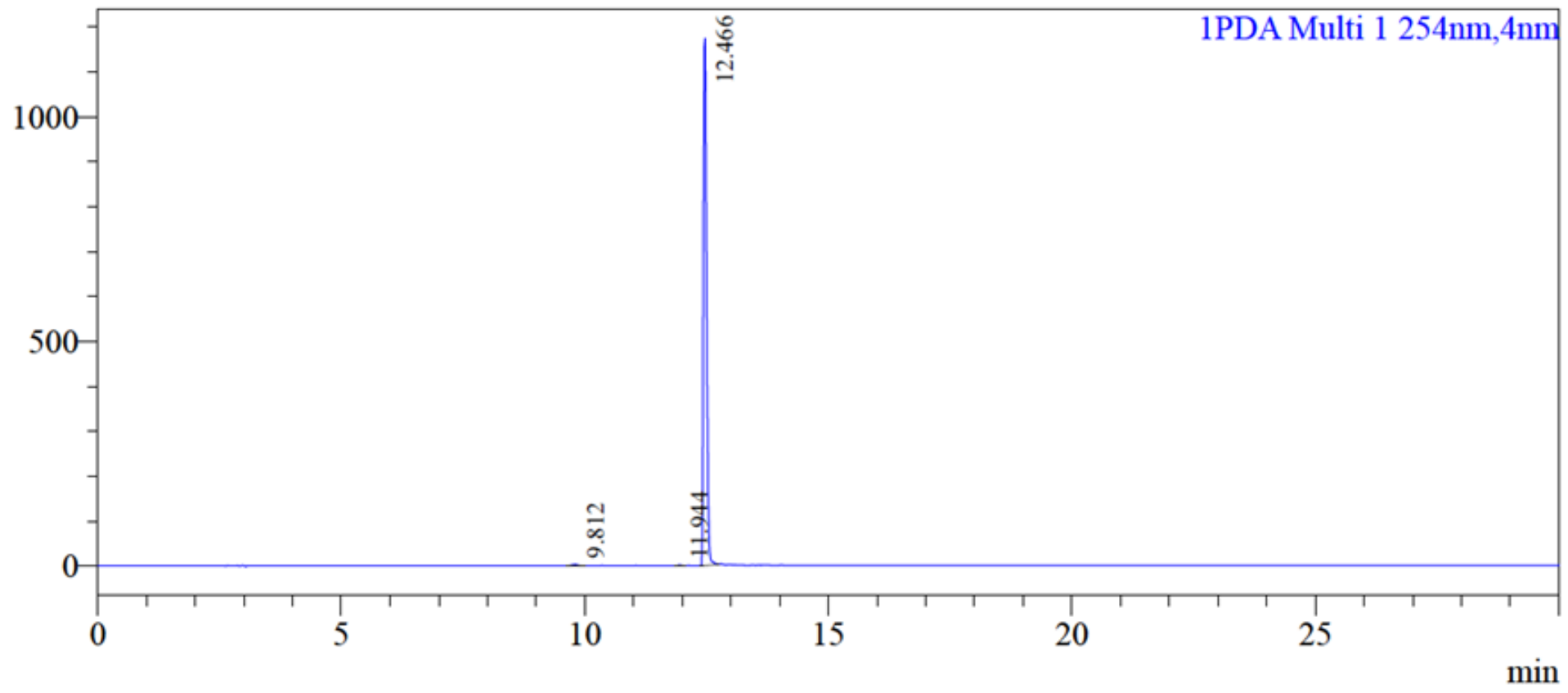

Peak Table

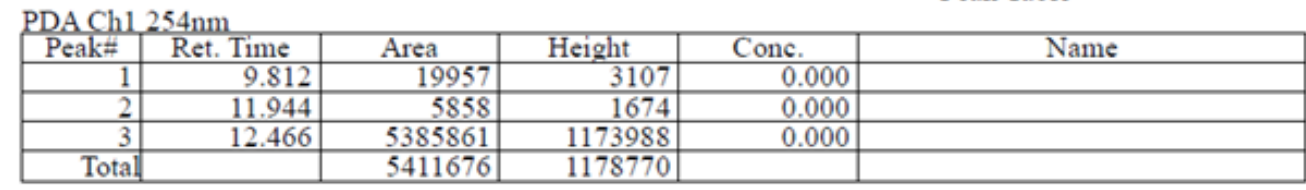

Figure S152: RP-HPLC trace for 41. 


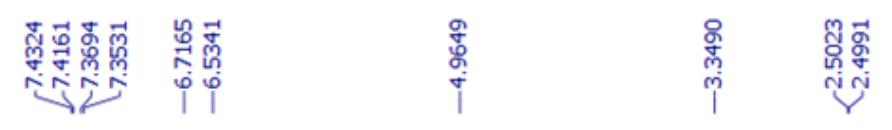

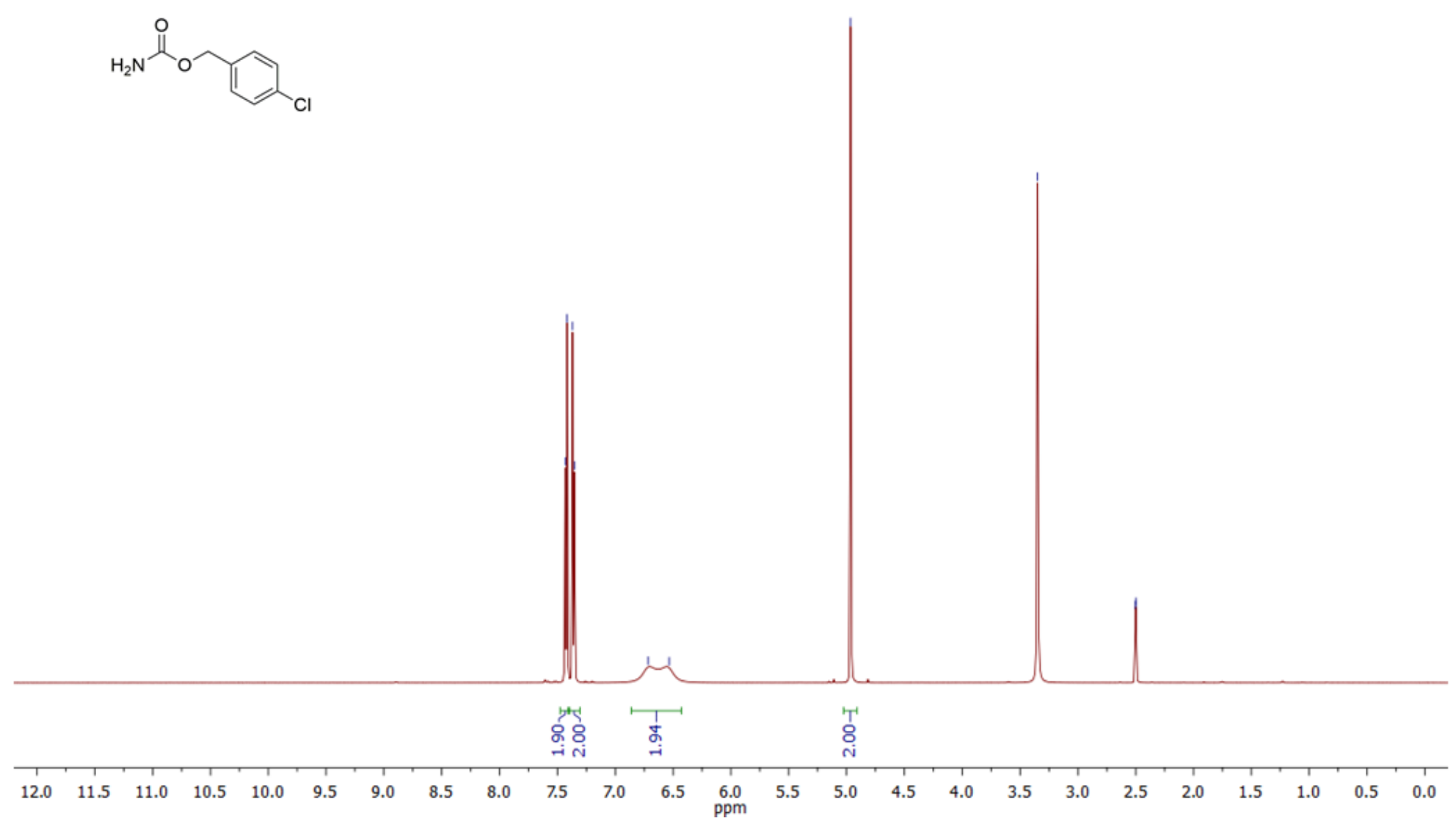

Figure S153: ${ }^{1} \mathrm{H}$ NMR (500 MHz) spectrum of 42 in DMSO- $d_{6}$. 

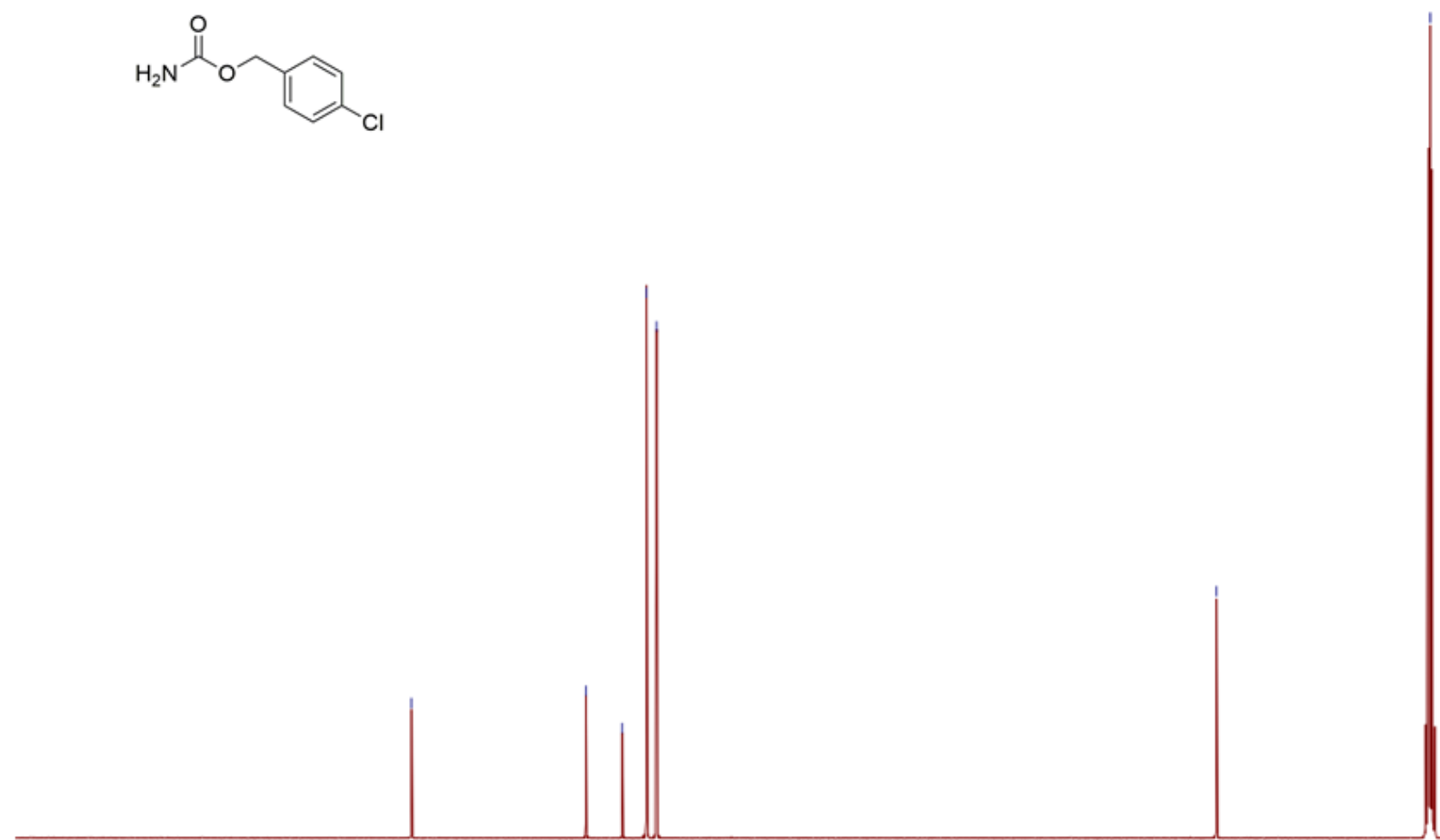

T $190-180-170$
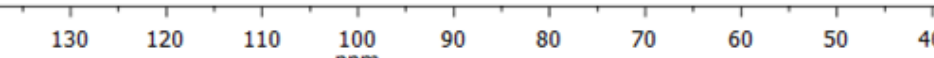

Figure S154: ${ }^{13} \mathrm{C}$ NMR (125 MHz) spectrum of 42 in DMSO- $d_{6}$ 
Chromatogram

SMH12-113A SMH12-113A.lcd

mAU

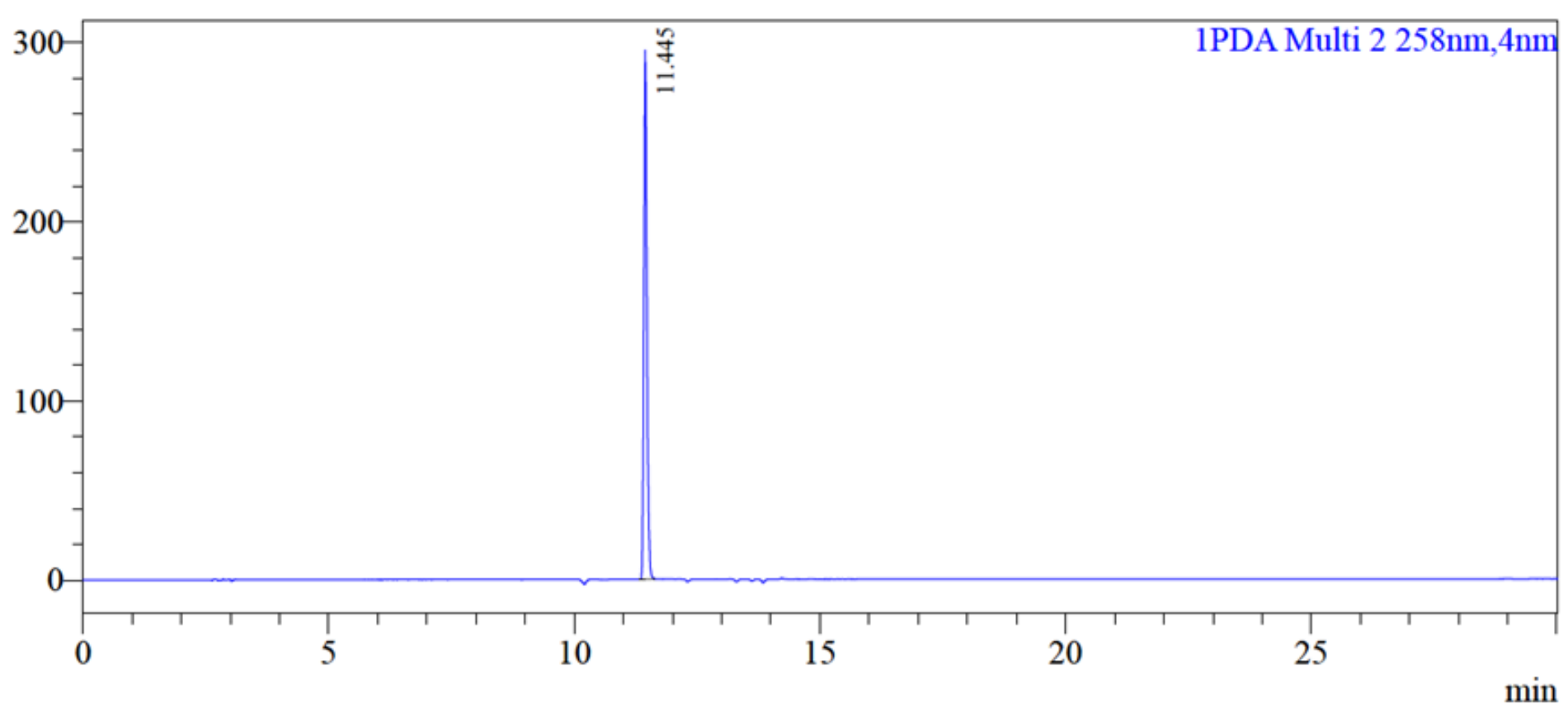

Peak Table

PDA Ch2 $258 \mathrm{~nm}$
\begin{tabular}{|r|r|r|r|r|r|}
\hline Peak $=$ & Ret. Time & \multicolumn{1}{|c|}{ Area } & Height & Conc. & Name \\
\hline 1 & 11.445 & 1281642 & 295344 & 100.000 & \\
\hline Total & & 1281642 & 295344 & & \\
\hline
\end{tabular}

Figure S155: RP-HPLC trace for $\mathbf{4 2 .}$ 


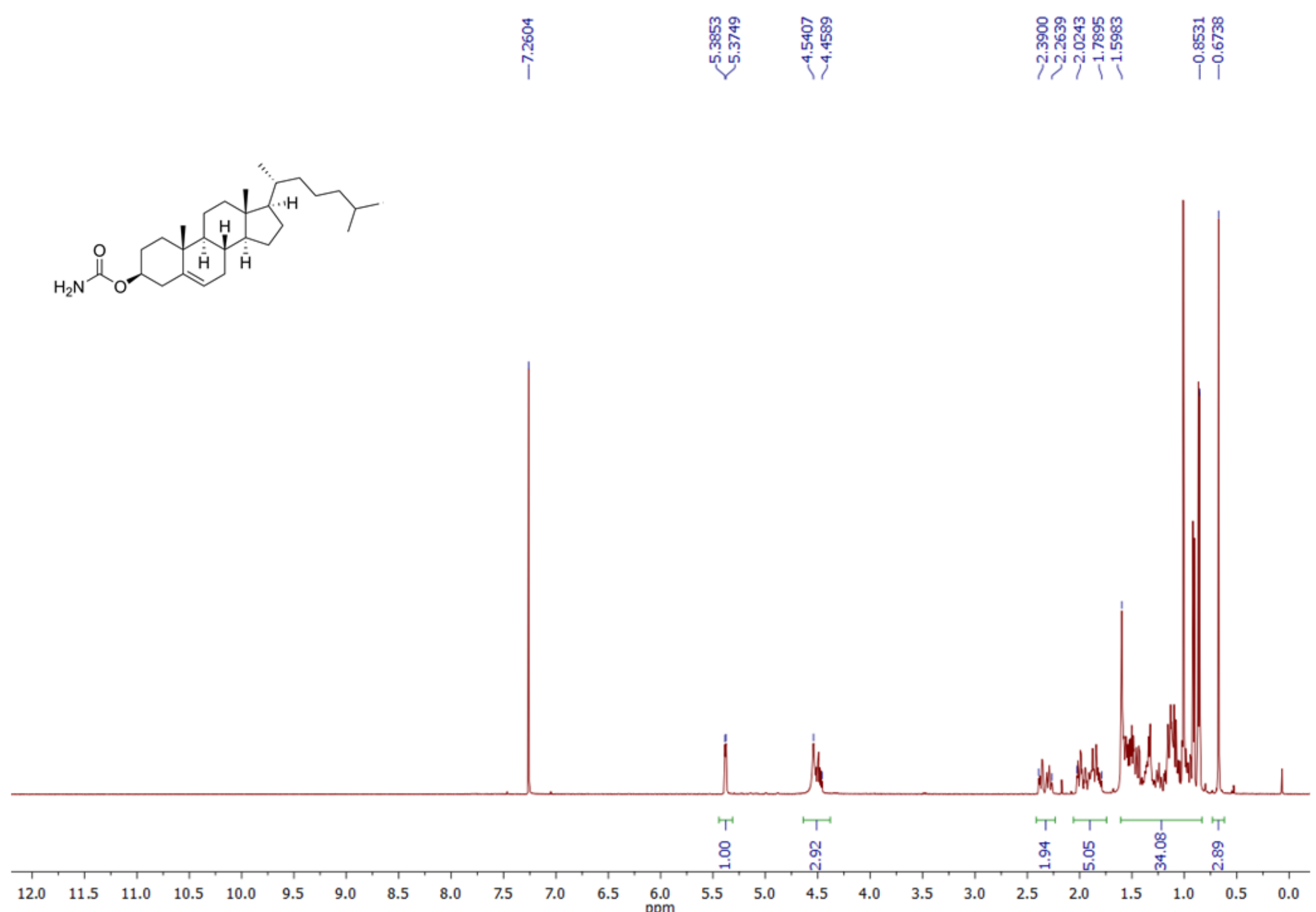

Figure S156: ${ }^{1} \mathrm{H}$ NMR (500 MHz) spectrum of 43 in $\mathrm{CDCl}_{3}$. 

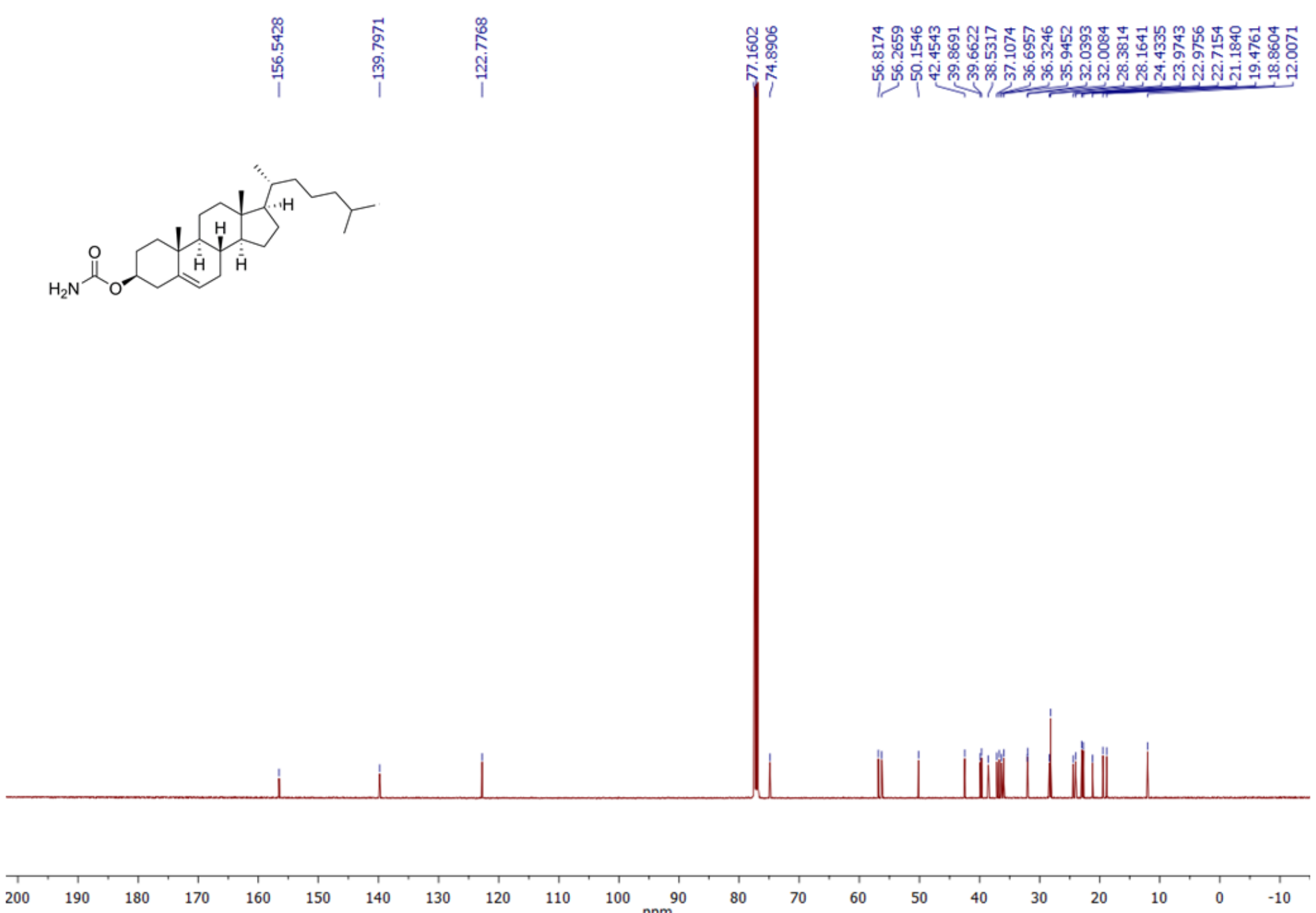

Figure S157: ${ }^{13} \mathrm{C}$ NMR (125 MHz) spectrum of 43 in $\mathrm{CDCl}_{3}$. 


\section{Crystallography Data}

A single crystal of $\mathbf{4 1}$ was mounted under paratone-N oil on a MiTeGen crystal mount, and X-ray diffraction data were collected at $150(2) \mathrm{K}$ with Mo Ka radiation $(\lambda=0.7107 \AA)$ on an Oxford Diffraction X-calibur small molecule diffractometer.[1] The data sets were corrected for absorption, the structures solved by direct methods using SHELXS and refined by full matrix least-squares on $\mathrm{F}^{2}$ by SHELXL, interfaced through the programs X-Seed and/or Olex.[2-5] In general, all non-hydrogen atoms were refined anisotropically, and hydrogen atoms were included as invariants at geometrically estimated positions. Details of data collection and structure refinement are given below (Table S1). CCDC deposition number 1975501 contains the supplementary crystallographic data for this paper. These data can be obtained free of charge from The Cambridge Crystallographic Data Centre via www.ccdc.cam.ac.uk/data_request/cif.

The structure of $\mathbf{4 1}$ packs in the chiral space group $P c a 2_{1}$ although the molecule itself is not chiral. The absolute structure of this particular crystal (the sample is a mixture of crystals possessing both chiral packing arrangements) was determined by anomalous dispersion effects using Mo K $\alpha$ radiation. When the reported enantiomer is chosen the Flack parameter is 0.1(2) for the refined structure and the Hooft parameter is 0.1(2). Further support for the absolute structure comes from analysing the Bijvoet pair data; the slope in a Bijvoet plot is positive (1.059) with P2(true) $=1.000$ and P3(true) 0.819, suggesting the correct enantiomer has been assigned.[6] 
Table S1: X-ray experimental data for 41.

\begin{tabular}{|c|c|}
\hline Compound & 41 \\
\hline Identification code & 41 \\
\hline $\mathrm{CCDC} \#$ & 1975501 \\
\hline Empirical formula & $\mathrm{C}_{18} \mathrm{H}_{15} \mathrm{NO}_{4}$ \\
\hline Formula weight & 309.31 \\
\hline Crystal system & orthorhombic \\
\hline Space group & $\operatorname{Pca}_{1}$ \\
\hline $\mathrm{a} / \AA$ & $15.0604(2)$ \\
\hline $\mathrm{b} / \AA$ & $14.2902(2)$ \\
\hline $\mathrm{c} / \AA ̊$ & $6.85110(10)$ \\
\hline$\alpha /{ }^{\circ}$ & 90 \\
\hline$\beta /{ }^{\circ}$ & 90 \\
\hline$\gamma /{ }^{\circ}$ & 90 \\
\hline Volume $/ \AA^{3}$ & $1474.47(4)$ \\
\hline $\mathrm{Z}$ & 4 \\
\hline$\rho_{\text {calc }} \mathrm{g} / \mathrm{cm}^{3}$ & 1.393 \\
\hline$\mu / \mathrm{mm}^{-1}$ & 0.099 \\
\hline $\mathrm{F}(000)$ & 648.0 \\
\hline Crystal size $/ \mathrm{mm}^{3}$ & $0.4 \times 0.35 \times 0.2$ \\
\hline $2 \Theta$ range for data collection ${ }^{\circ}$ & 7.13 to 58.728 \\
\hline Reflections collected & 62797 \\
\hline Independent reflections & $3779\left[R_{\text {int }}=0.0343, R_{\text {sigma }}=0.0134\right]$ \\
\hline Data/restraints/parameters & $3779 / 1 / 210$ \\
\hline Goodness-of-fit on $\mathrm{F}^{2}$ & 1.047 \\
\hline Final $R$ indexes $[\mathrm{I}>=2 \sigma(\mathrm{I})]$ & $\mathrm{R}_{1}=0.0339, \mathrm{wR}_{2}=0.0880$ \\
\hline Final R indexes [all data] & $\mathrm{R}_{1}=0.0377, \mathrm{wR}_{2}=0.0904$ \\
\hline Largest diff. peak/hole / e $\AA^{-3}$ & $0.26 /-0.21$ \\
\hline Flack parameter & $0.1(2)$ \\
\hline
\end{tabular}




\section{Gel Fabrication}

\section{Method}

The coumarin acrylate hydrogel was prepared from a mixture of 2-hydroxyethylmethacrylate (HEMA) $(1 \mathrm{~mL})$, ethylene glycol dimethacrylate $(20 \mu \mathrm{L}), N, N, N, N$-tetramethylethane-1,2-diamine $(10 \mu \mathrm{L})$, potassium persulfate ( $160 \mu \mathrm{L}$ of $100 \mathrm{mM}$ solution) and 20 ( $800 \mu \mathrm{L}$ of $10 \mathrm{mM}$ solution in DMSO). The monomer solution was thoroughly mixed and cast between two acrylic sheets spaced $1.5 \mathrm{~mm}$ apart. The hydrogel was polymerised at $55{ }^{\circ} \mathrm{C}$ for $2 \mathrm{~h}$, then allowed to cure at room temperature for overnight. The hydrogel was removed from the cast and soaked in ultrapure water for $2 \mathrm{~h}$ to remove unreacted monomers. The final hydrogel was a flexible and transparent freestanding material.

A coumarin propylamide hydrogel was prepared by the same method, substituting $\mathbf{2 1}$ as the coumarin additive. A negative control (non-fluorescent) hydrogel was also prepared using $800 \mu \mathrm{L}$ of DMSO with no coumarin compound present.

\section{Measurement}

Fluorescence emission from the hydrogels was recorded on a Varian Cary Eclipse fluorescence spectrophotometer. The hydrogel was placed across the diagonal of a quartz cuvette as shown in Figure S154, to minimize reflection of the excitation light into the detector. An excitation wavelength of $325 \mathrm{~nm}$ was used and the emission was recorded over the range of 350-600 nm.

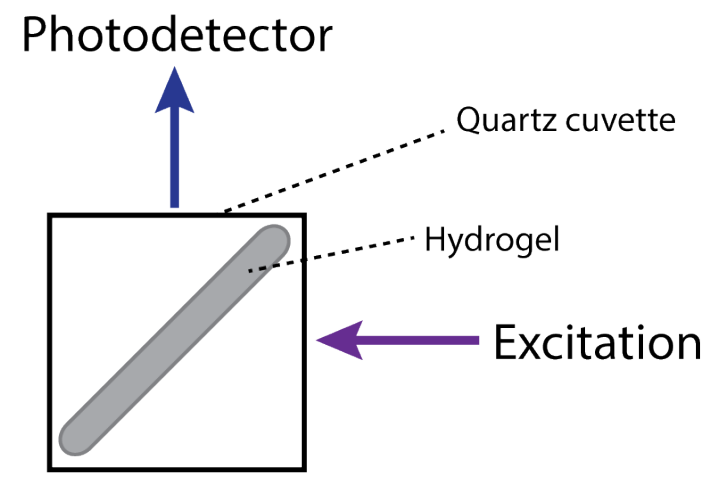

Figure S158: Top-down view of the hydrogel position in the cuvette for fluorescence measurements. 
Table S2: Photophysical data of $\mathbf{2 5}$ in different solvents.

\begin{tabular}{|c|c|c|c|c|c|c|c|c|}
\hline \multirow[t]{3}{*}{ Entry } & \multirow[t]{3}{*}{ Solvent } & \multicolumn{2}{|c|}{ Absorption Properties } & \multicolumn{5}{|c|}{ Emission Properties } \\
\hline & & \multirow{2}{*}{$\begin{array}{l}\lambda_{\mathrm{abs}} \\
(\mathrm{nm})\end{array}$} & \multirow{2}{*}{$\begin{array}{c}\varepsilon_{\max } \\
\left(\mathrm{M}^{-1} \mathrm{~cm}^{-1}\right)\end{array}$} & \multirow{2}{*}{$\begin{array}{c}\lambda_{\mathrm{em}} \\
(\mathrm{nm})\end{array}$} & \multicolumn{2}{|c|}{ Stokes shift } & \multirow[t]{2}{*}{$\Phi_{\mathrm{F}}$} & \multirow{2}{*}{$\begin{array}{l}\text { Brightness } \\
\left(\mathrm{M}^{-1} \mathrm{~cm}^{-1}\right)\end{array}$} \\
\hline & & & & & $(\mathrm{nm})$ & $\left(\mathrm{cm}^{-1}\right)$ & & \\
\hline 1 & $\begin{array}{l}1 \% \text { DMSO } \\
\text { in } \mathrm{H}_{2} \mathrm{O}\end{array}$ & 327 & 15,600 & 403 & 76 & 5,767 & 0.94 & 14,600 \\
\hline 2 & DMSO & 330 & 18,000 & 389 & 59 & 4,596 & 0.49 & 8,900 \\
\hline 3 & $\mathrm{CHCl}_{3}$ & 329 & 15,600 & 389 & 60 & 4,688 & 0.32 & 5,000 \\
\hline 4 & $\mathrm{PhMe}$ & 327 & 15,400 & 382 & 55 & 4,403 & 0.04 & 600 \\
\hline
\end{tabular}




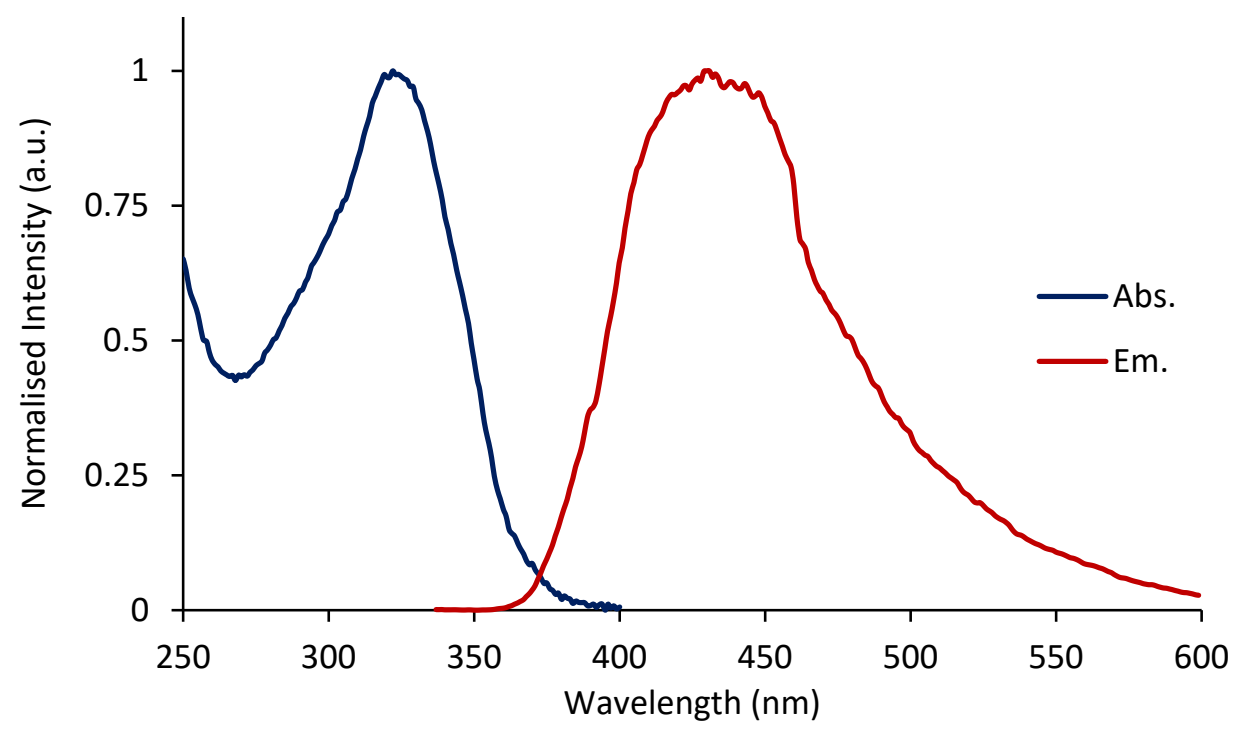

Figure S159: Normalised absorbance and fluorescence emission spectra for $\mathbf{1}$.

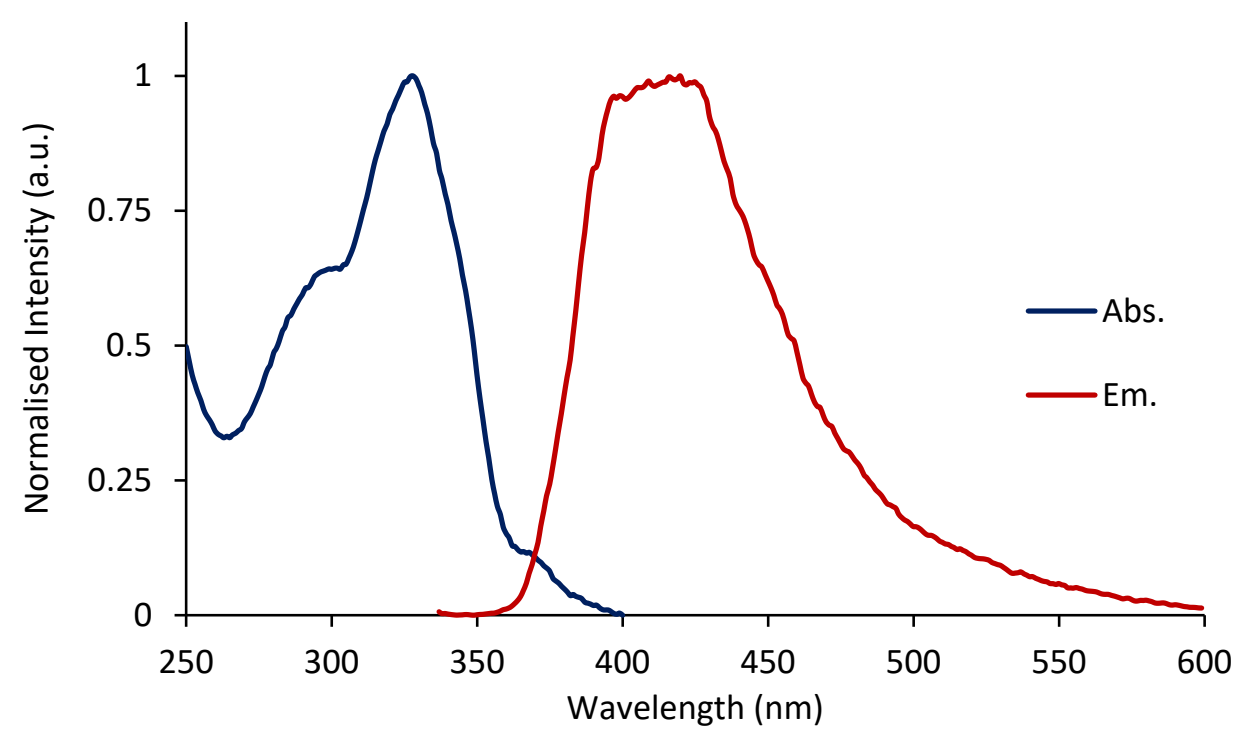

Figure S160: Normalised absorbance and fluorescence emission spectra for 11. 


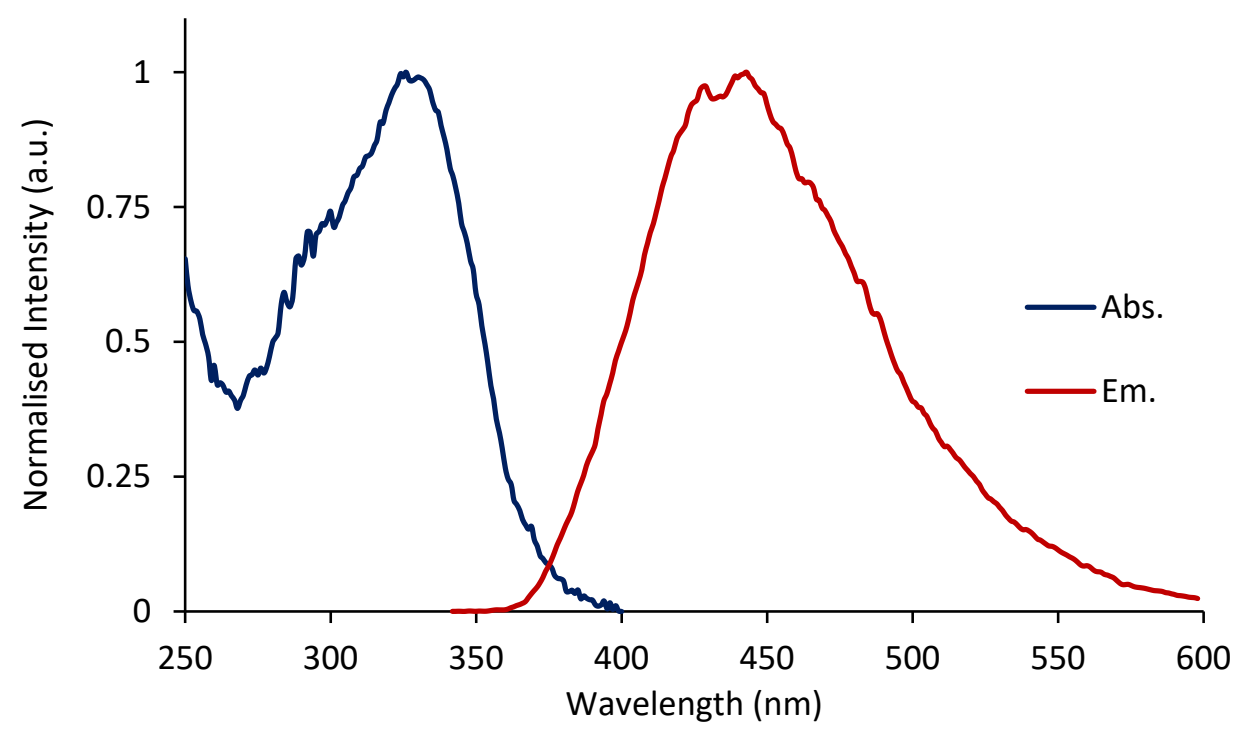

Figure S161: Normalised absorbance and fluorescence emission spectra for 12.

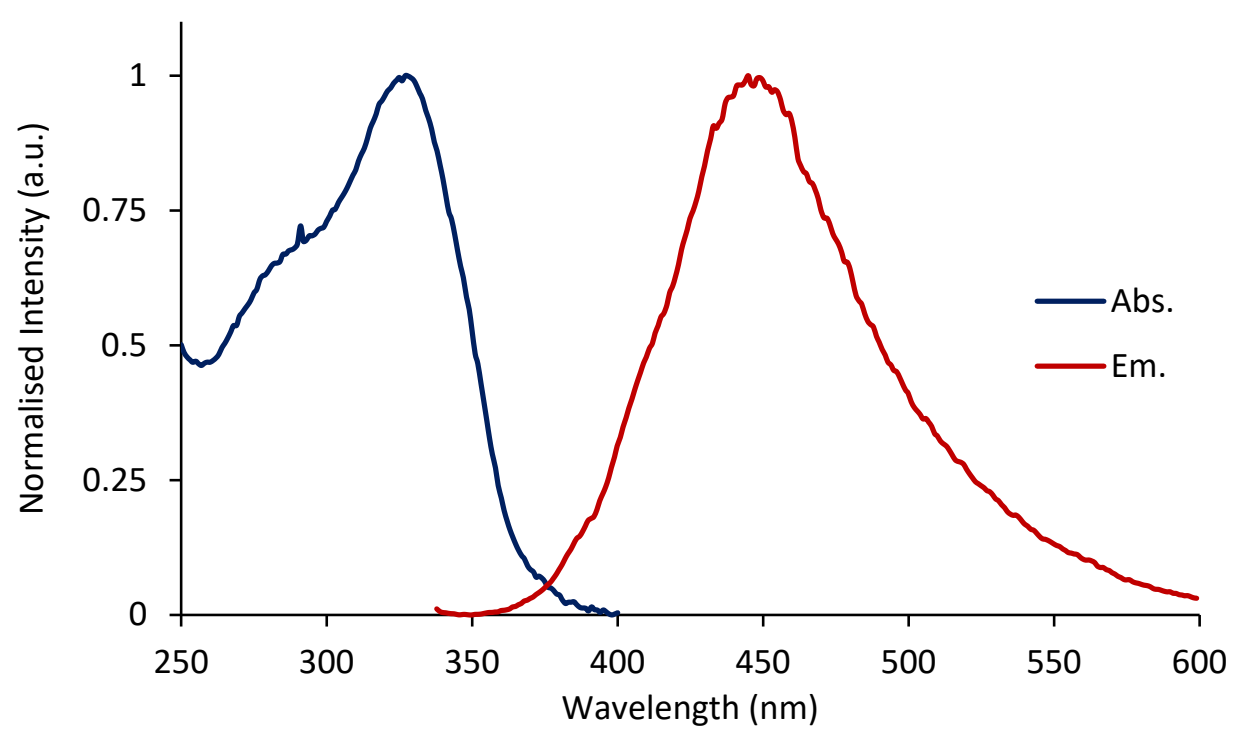

Figure S162: Normalised absorbance and fluorescence emission spectra for 17. 


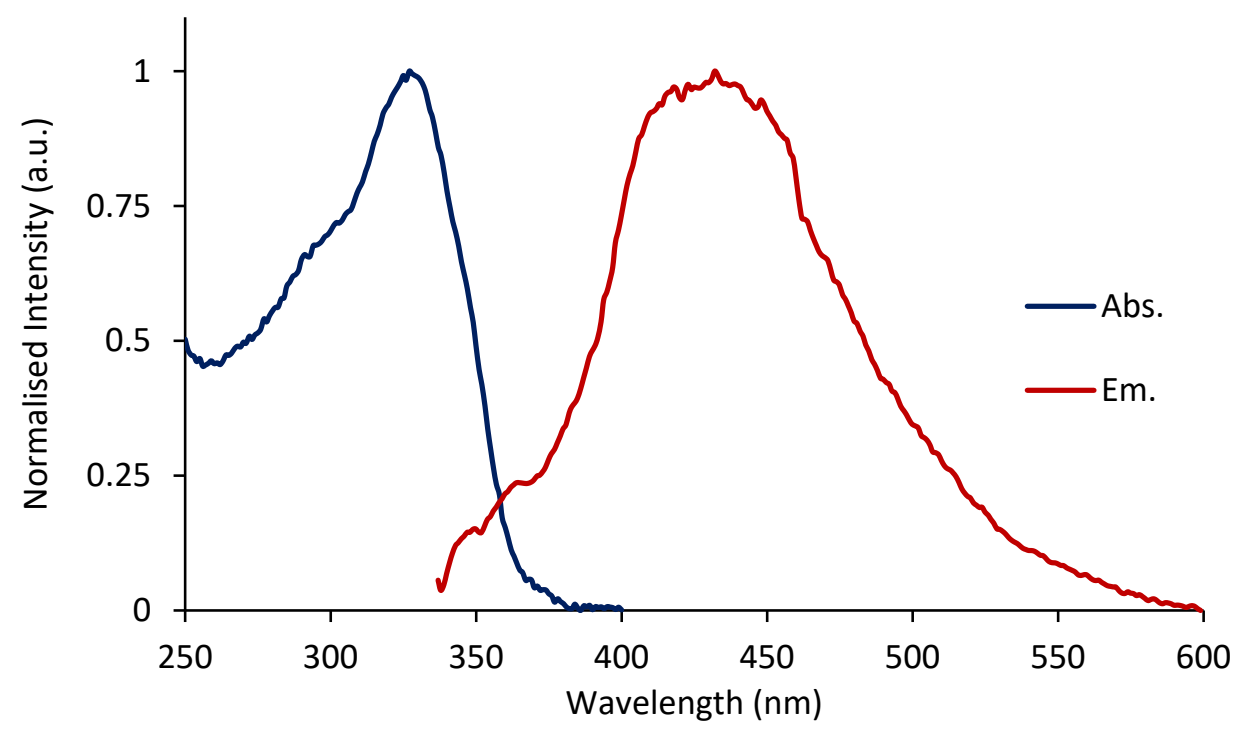

Figure S163: Normalised absorbance and fluorescence emission spectra for $\mathbf{1 8 .}$

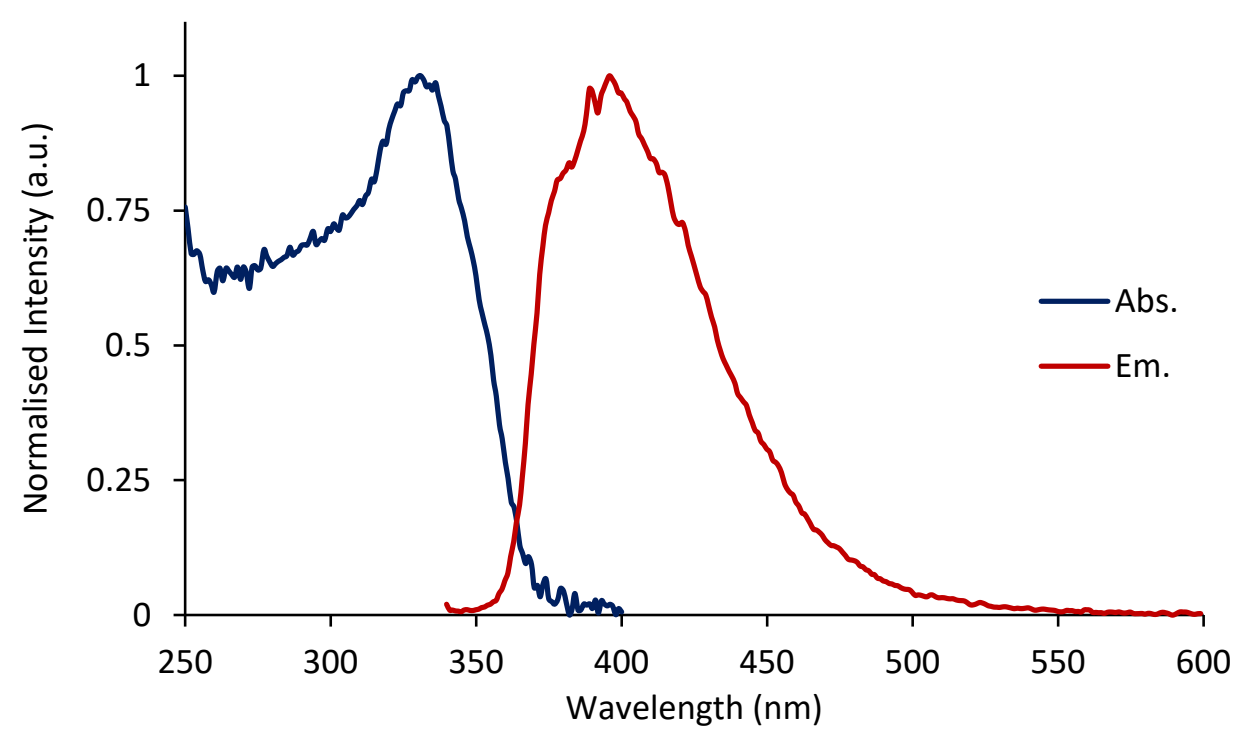

Figure S164: Normalised absorbance and fluorescence emission spectra for 19. 


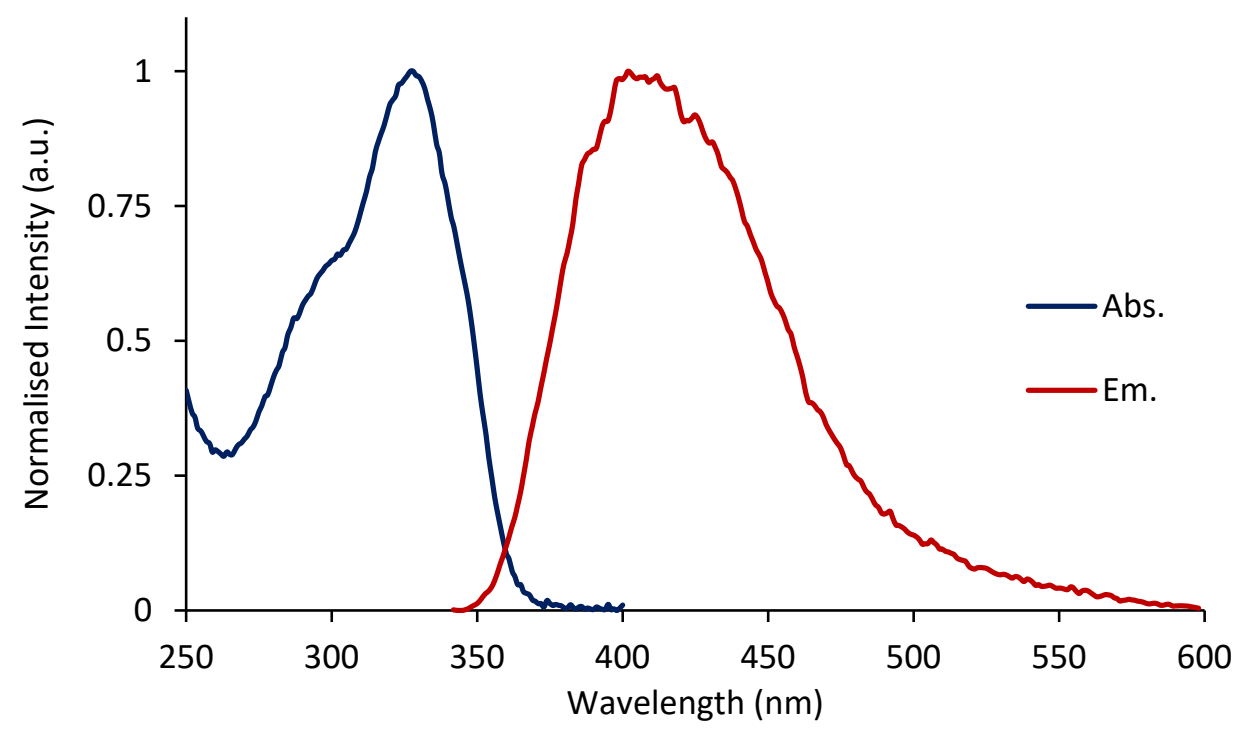

Figure S165: Normalised absorbance and fluorescence emission spectra for $\mathbf{2 0 .}$

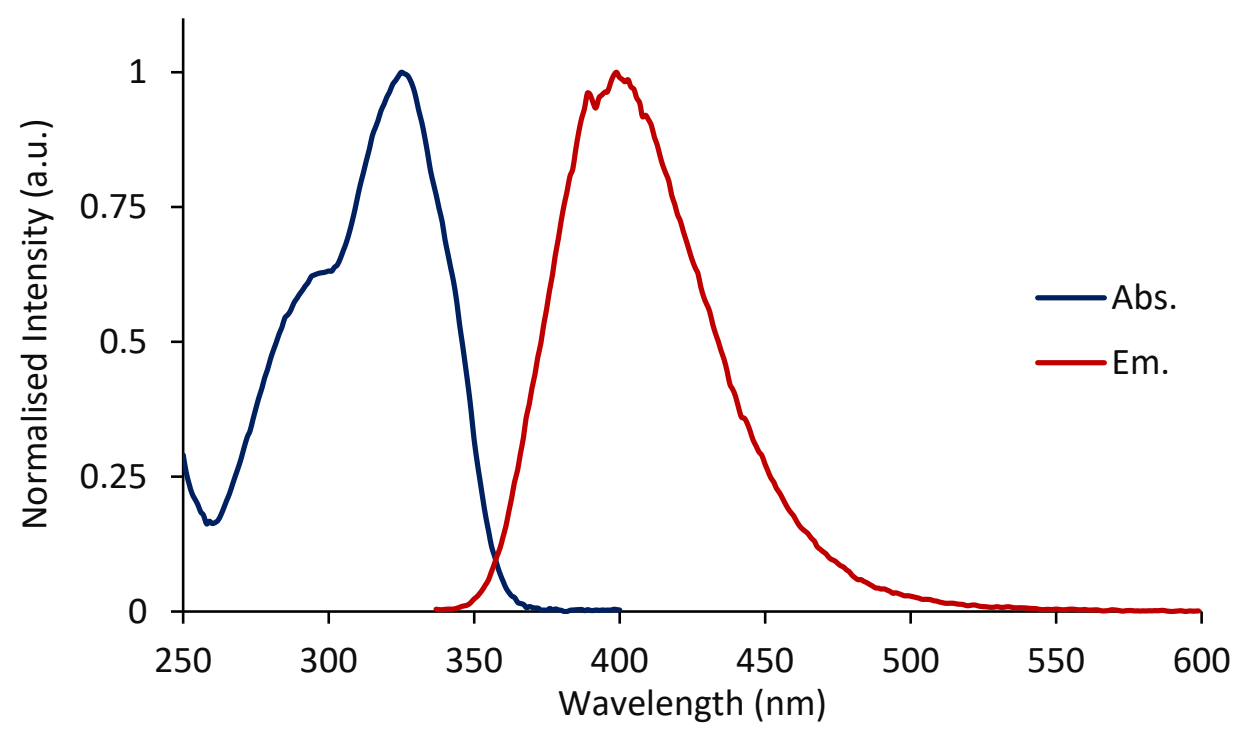

Figure S166: Normalised absorbance and fluorescence emission spectra for 21. 


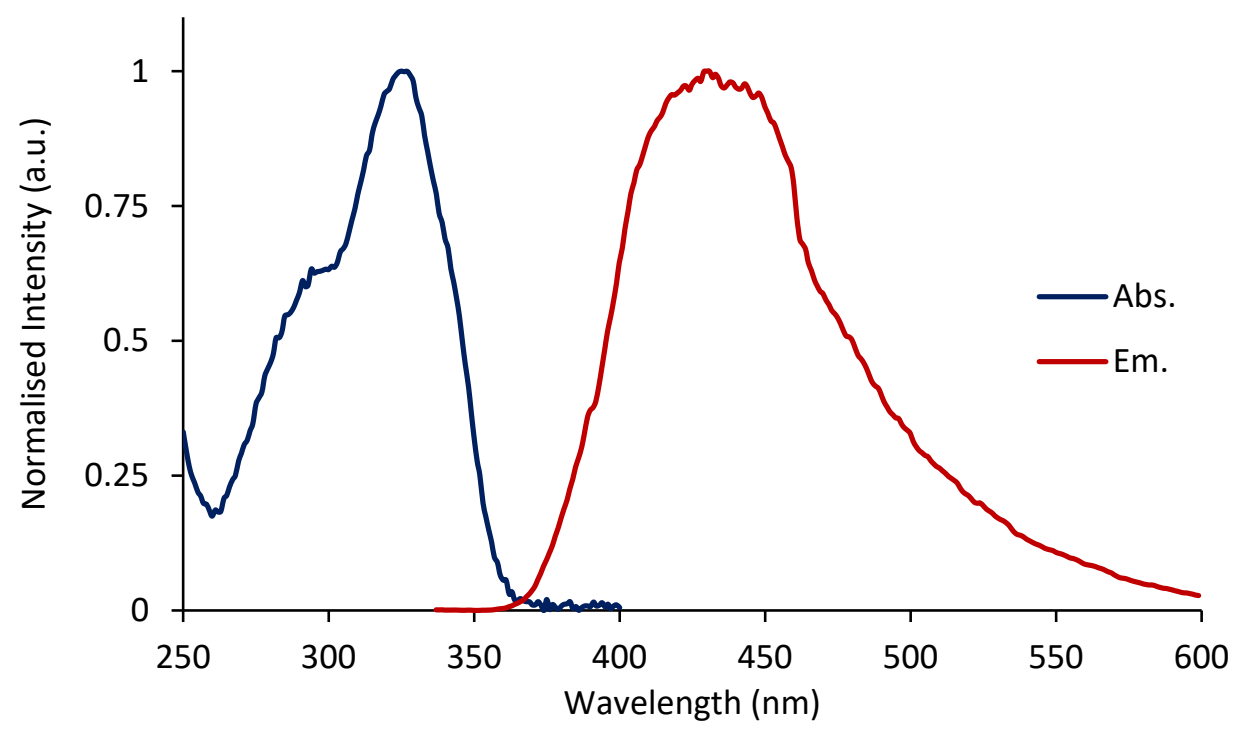

Figure S167: Normalised absorbance and fluorescence emission spectra for 22.

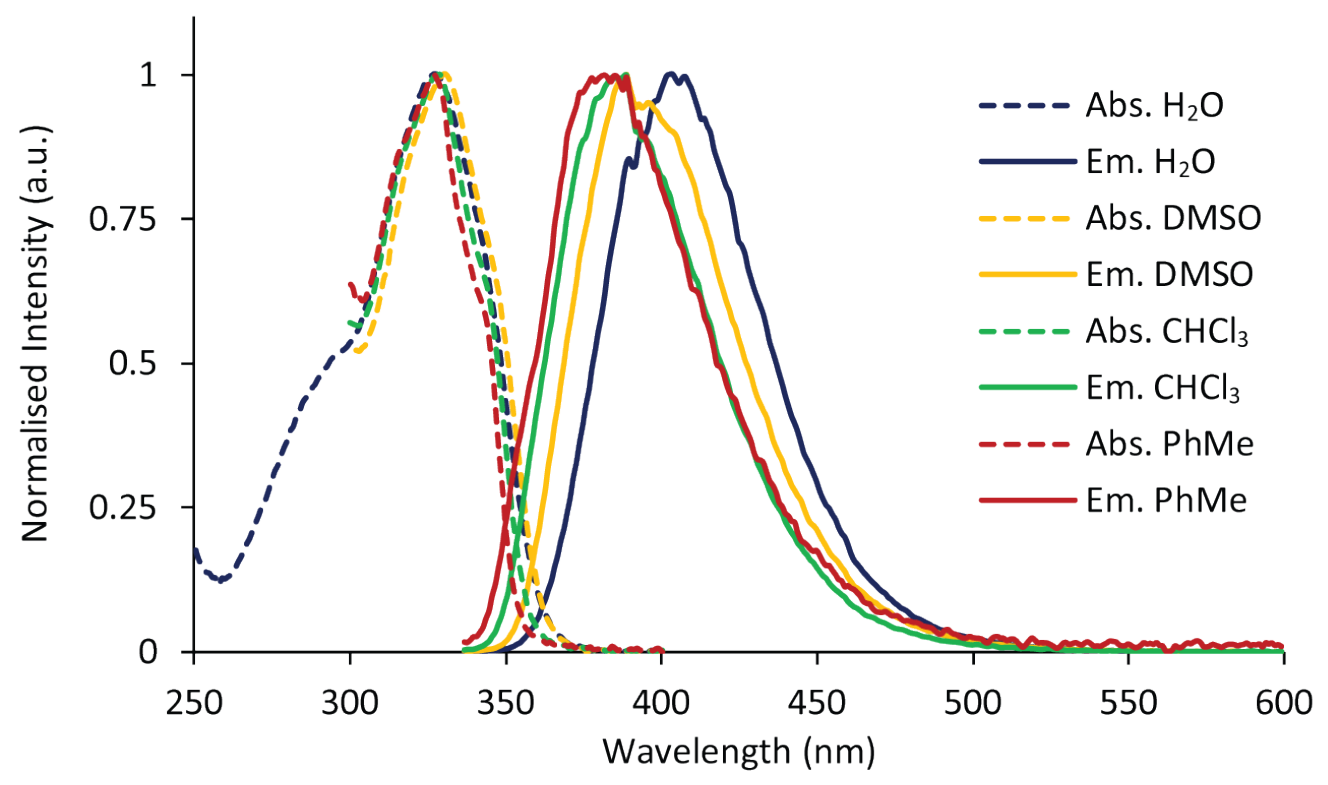

Figure S168: Normalised absorbance and fluorescence emission spectra for $\mathbf{2 5}$ in different solvents. 


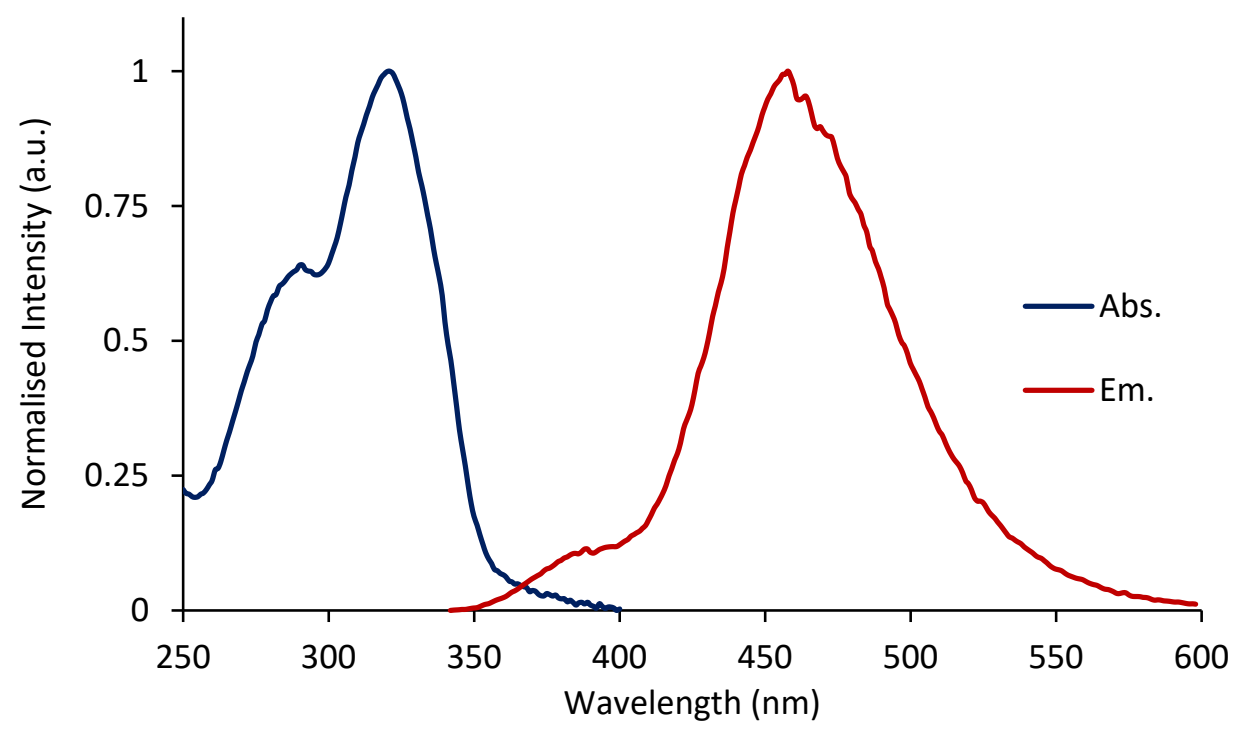

Figure S169: Normalised absorbance and fluorescence emission spectra for 29.

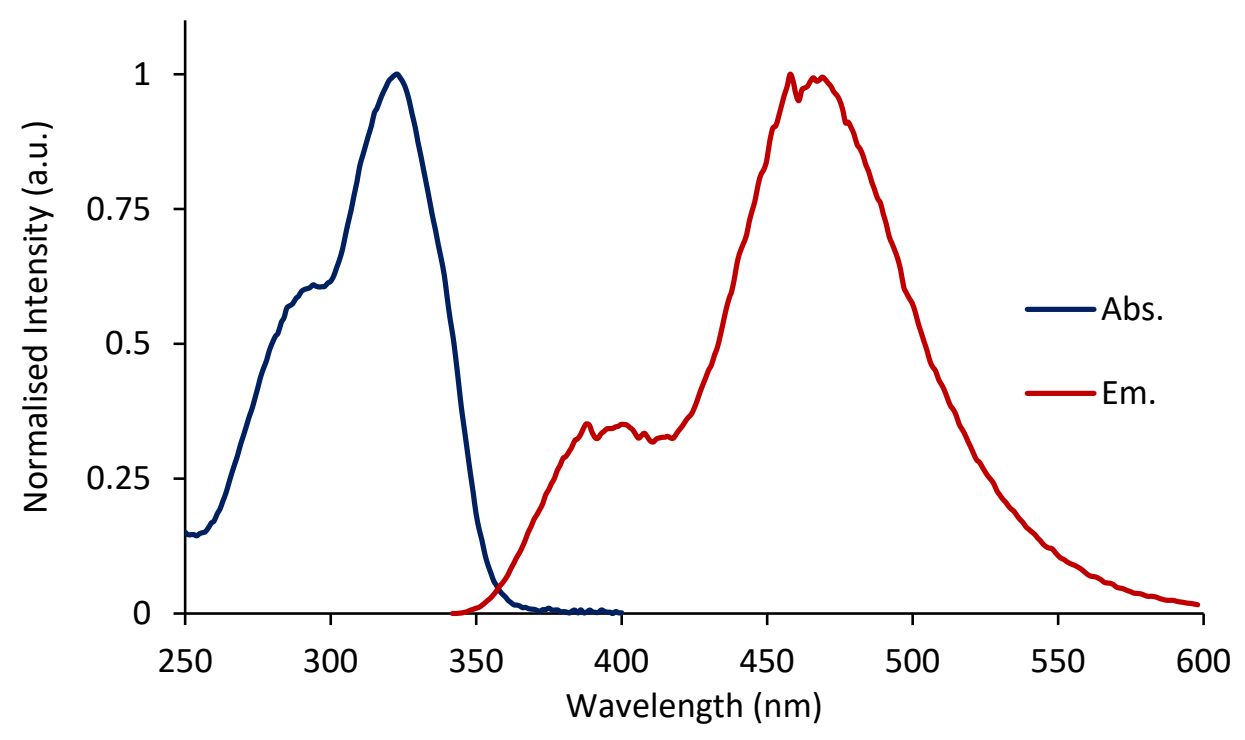

Figure S170: Normalised absorbance and fluorescence emission spectra for $\mathbf{3 0 .}$ 


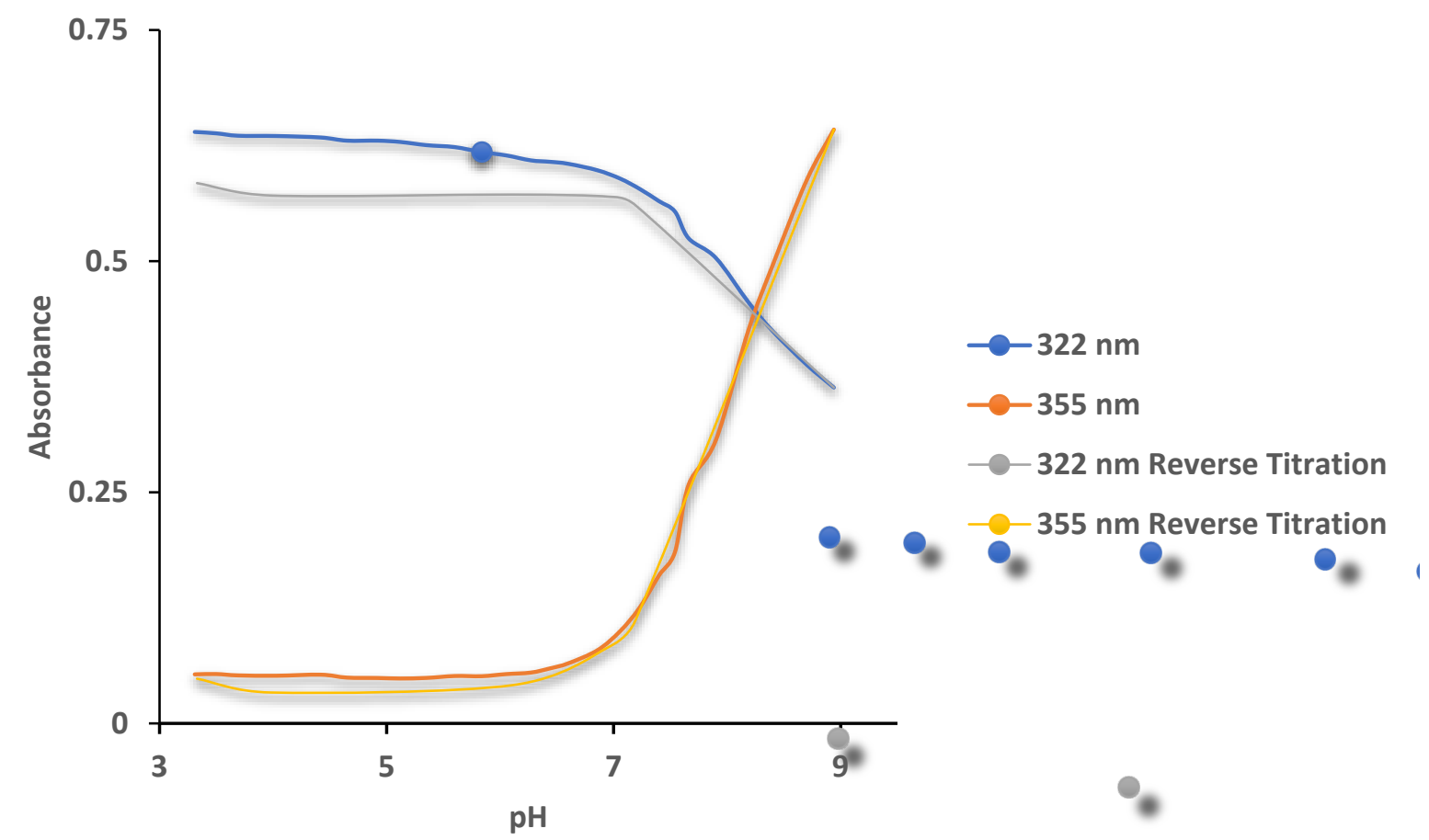

Figure S171: Absorbance versus $\mathrm{pH}$ at 322 and $355 \mathrm{~nm}$ wavelengths for $\mathbf{3 0}$ over $\mathrm{pH}$ range 3-9. Both the forward and reverse titrations are depicted.

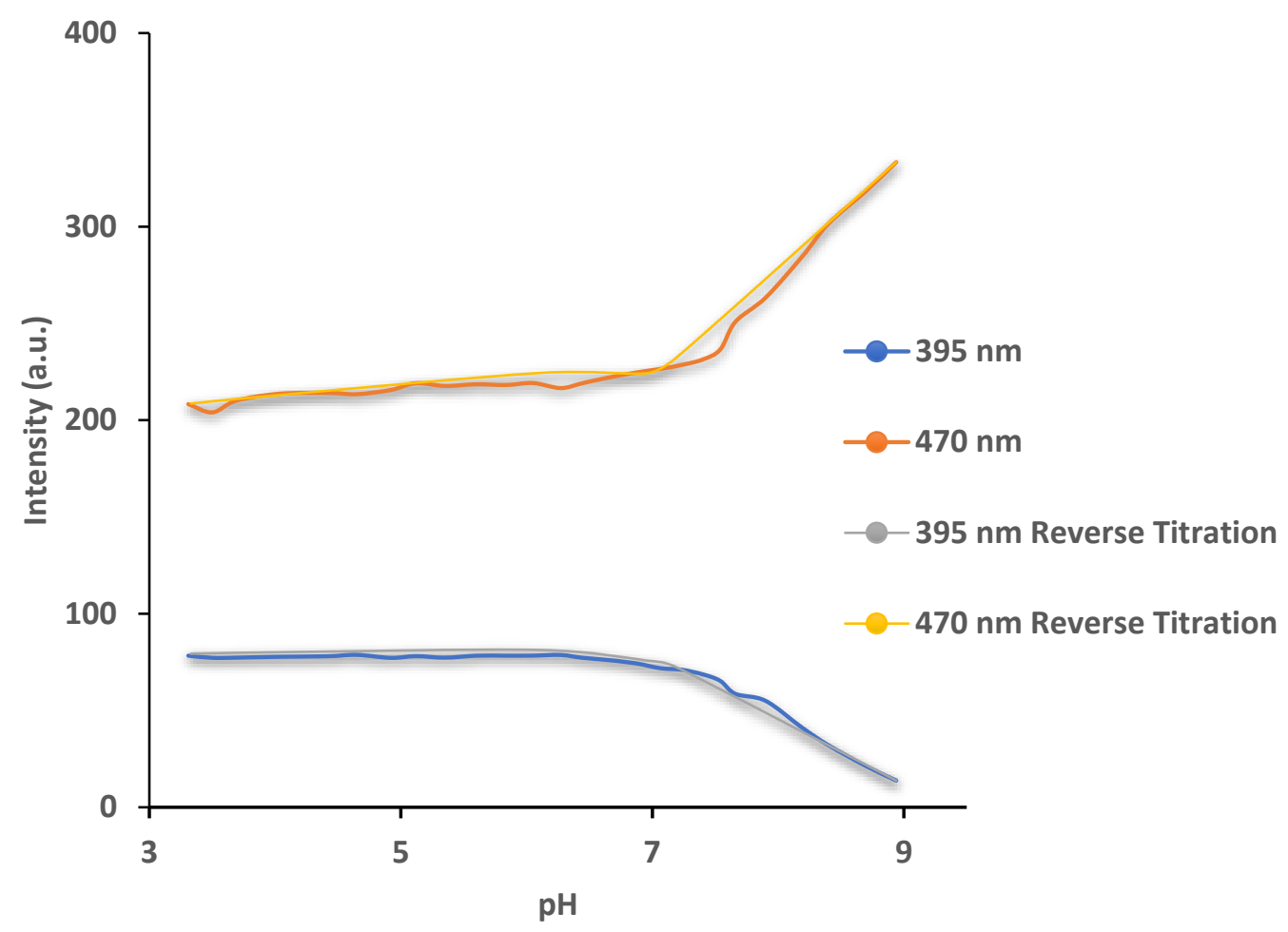

Figure S172: Emission (excitation at $322 \mathrm{~nm}$ ) versus pH at 395 and $470 \mathrm{~nm}$ wavelengths for 30 over pH range $3-9$. Both the forward and reverse titrations are depicted. 


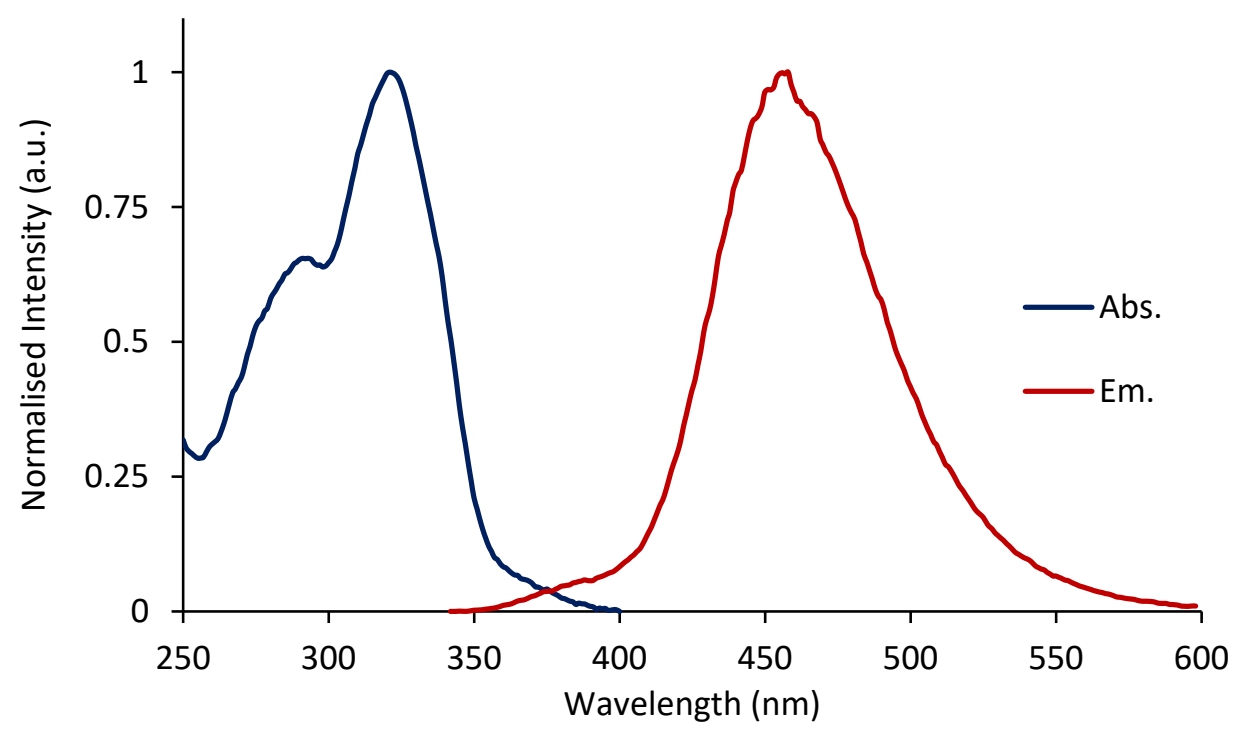

Figure S173: Normalised absorbance and fluorescence emission spectra for 31.

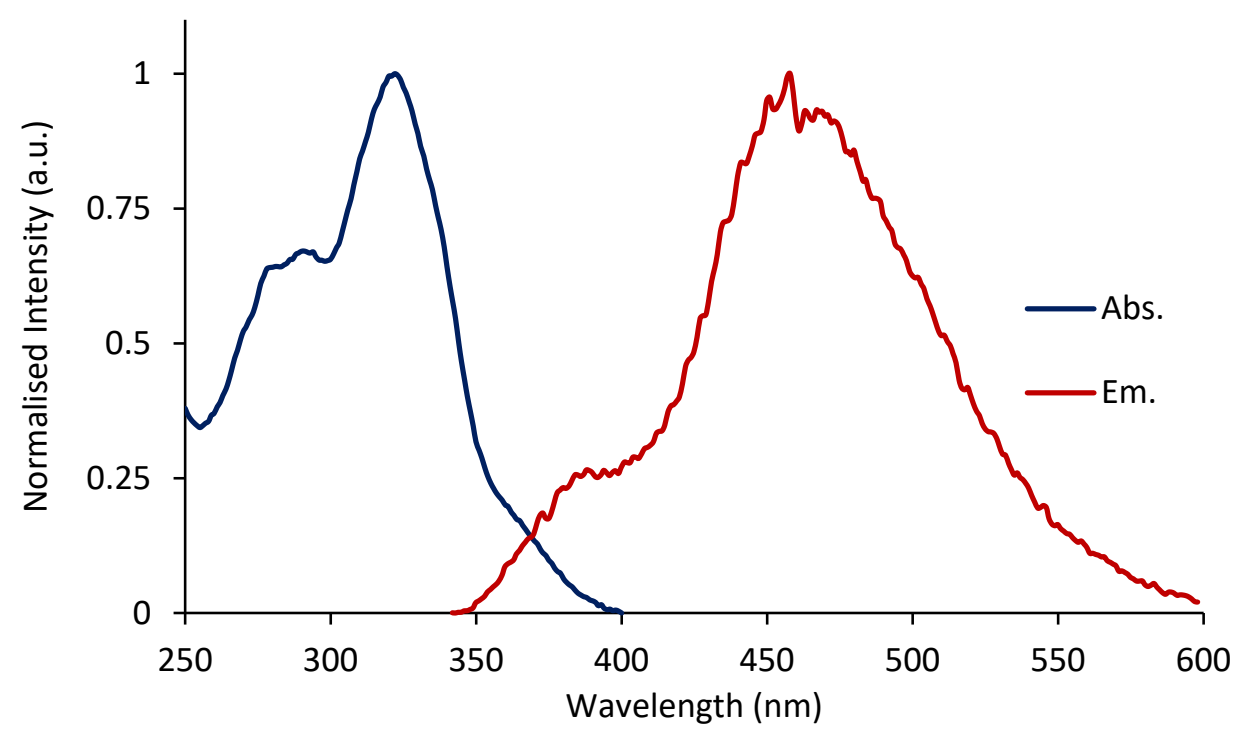

Figure S174: Normalised absorbance and fluorescence emission spectra for 33. 


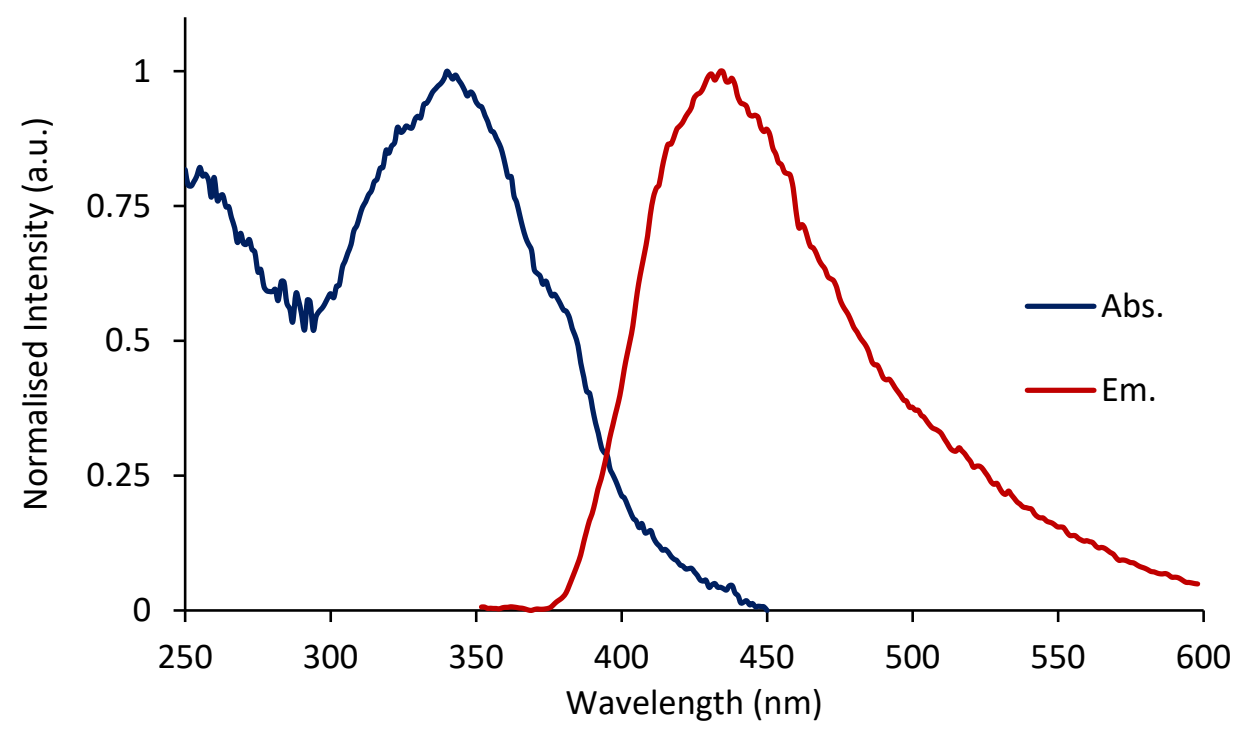

Figure S175: Normalised absorbance and fluorescence emission spectra for 39.

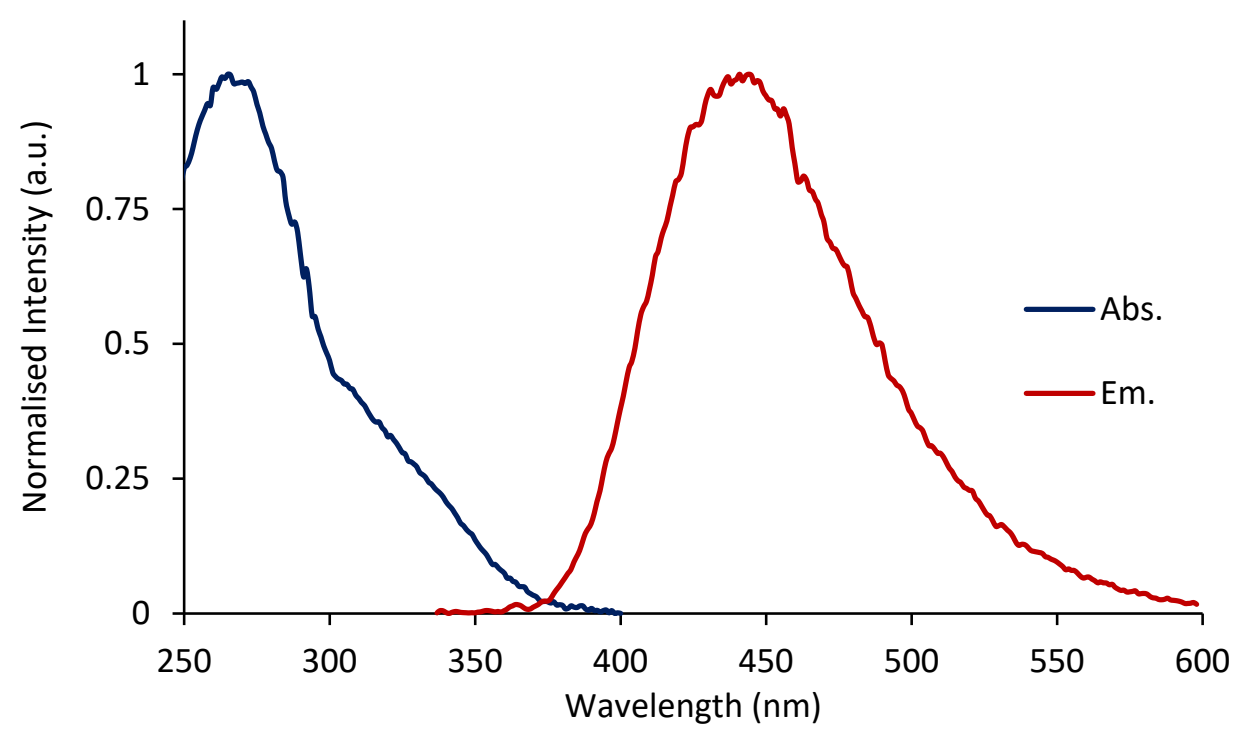

Figure S176: Normalised absorbance and fluorescence emission spectra for $\mathbf{4 1 .}$ 


\section{References}

[1] CrysAlisPro, CrysAlis171.NET, Version 1.171.34.44, Oxford Diffraction Ltd: Oxfordshire, UK, 2010.

[2] Sheldrick, G. M. Phase annealing in SHELX-90: Direct methods for larger structures. Acta Crystallogr. A 1990, 46, 467-73.

[3] Sheldrick GM. SHELXL-2014, University of Göttingen, Göttingen, Germany, 2014.

[4] Barbour, L. J. X-Seed - A software tool for supramolecular crystallography. J. Supramol. Chem. 2001, 1, 189-91.

[5] Dolomanov, O. V.; Bourhis, L. J.; Gildea, R. J.; Howard, J. A.; Puschmann, H. OLEX2: a complete structure solution, refinement and analysis program. J. Appl. Crystallogr. 2009, 42, 339-41.

[6] Hooft, R. W.; Straver, L. H.; Spek, A. L. Determination of absolute structure using Bayesian statistics on Bijvoet differences. J. Appl. Crystallogr. 2008, 41, 96-103. 